\title{
A $\boldsymbol{W H} \rightarrow \tau \nu b \bar{b}$ Higgs Search with the $\mathrm{D} \varnothing$ Detector at the Tevatron Or
}

\author{
How to Find the Higgs at the End \\ of a Circular Collider
}




\section{A $W H \rightarrow \tau \nu b \bar{b}$ HigGS SEARCH WITH THE D $\varnothing$ DETECTOR AT THE TEVATRON}

OR

How to Find the HigGs AT THE End OF A Circular Collider

\section{PROEFSCHRIFT}

TER VERKRIJGING VAN DE GRAAD VAN DOCTOR AAN DE RADBOUd UNIVERSITEIT NiJMEGEN

op GeZaG VAN DE RECTOR magnificus prof. mR. S. C. J. J. Kortmann, VOLGENS BESLUIT VAN HET COLLEGE VAN DECANEN

IN HET OPENBAAR TE VERDEDIGEN OP MAANDAG 25 JUNI 2012 OM 10.30 UUR PRECIES

DOOR

MELVIN MiCHAËL MEIJER 
Promotor:

Copromotor:

Manuscriptcommissie: Prof. DR. N. De Groot
Prof. DR. S. J. DE JONG

Dr. F. Filthaut

Prof. DR. P. H. E. Tiesinga

Prof. DR. S. SöldNER-REMBOLd

- The University of MAnchester

Prof. Dr. A. Kharchilava

- State University of New York, Buffalo

DR. P. FERRARI

- Nikhef, Amsterdam 


\section{Contents}

$\begin{array}{ll}\text { Introduction } & 1\end{array}$

\begin{tabular}{lll}
\hline 1 & Theory & 3
\end{tabular}

1.1 The Standard Model . . . . . . . . . . . . . . . . . . . . . . . 3

$1.1 .1 \quad$ A Short Introduction . . . . . . . . . . . . . . . . . . . . . 3

1.1 .2 Quantum Field Theory . . . . . . . . . . . . . . . . . 5

Feynman Diagrams. . . . . . . . . . . . . . . . 5

Local Gauge Theory . . . . . . . . . . . . . . . . 5

Non-Abelian Gauge Theories . . . . . . . . . . . . . . 6

Spontaneous Symmetry Breaking. . . . . . . . . . . . 7

1.2 The Higgs Boson $\ldots \ldots \ldots \ldots \ldots \ldots$

1.2 .1 Known Properties . . . . . . . . . . . . . . . . . . 10

Unitarity Bound . . . . . . . . . . . . . . . . . . . 10

Running Coupling Constraints . . . . . . . . . . . . 11

Indirect Measurements . . . . . . . . . . . . . . . . 13

Direct Searches . . . . . . . . . . . . . . . . . . 14

1.2 .2 At the Tevatron . . . . . . . . . . . . . . . 15

$\begin{array}{lll}2 & \text { Experimental Setup } & 19\end{array}$

2.1 Proton-Antiproton Collisions . . . . . . . . . . . . . . . 20

2.1 .1 Proton Source . . . . . . . . . . . . . . . . . . 20

$2.1 .2 \quad$ Main Injector, Recycler and Antiproton Source . . . . . . . 21

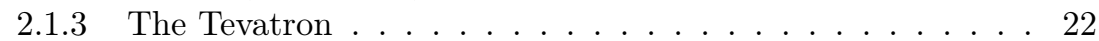

2.2 The $\mathrm{D} \emptyset$ Detector $\ldots \ldots \ldots \ldots \ldots \ldots$

2.2 .1 Transverse Momentum and Coordinate Systems. . . . . . . 24

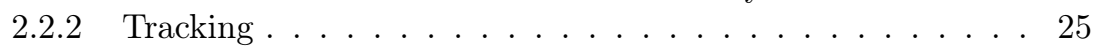

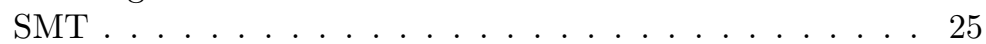

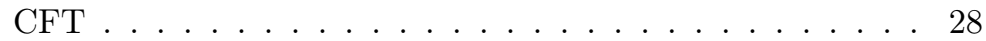

Preshower Detectors . . . . . . . . . . . . . . . . . . . . 29

2.2 .3 Calorimetry $\ldots \ldots \ldots \ldots \ldots$ 
Inter-Cryostat Detector and Massless Gaps . . . . . . . . . 32

2.2 .4 The Muon System . . . . . . . . . . . . . . . . . . 32

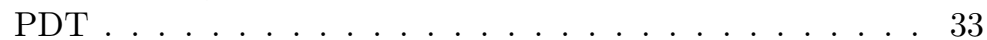

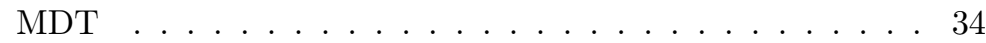

Scintillation Counters . . . . . . . . . . . . . . . 34

2.2 .5 Luminosity Measurement . . . . . . . . . . . . . . . . . . . 34

2.3 Triggering $\ldots \ldots \ldots \ldots \ldots \ldots \ldots$

2.3 .1 The Trigger Framework . . . . . . . . . . . . . . . 36

2.3 .2 Level 1 . . . . . . . . . . . . . . . . . . . . . . . . . 37

$2.3 .3 \quad$ Level 2

$2.3 .4 \quad$ Level 3 . . . . . . . . . . . . . . . . . . . . . . . . . . 38

2.4 Monte Carlo Simulations . . . . . . . . . . . . . . . . . . . . . . . . 38

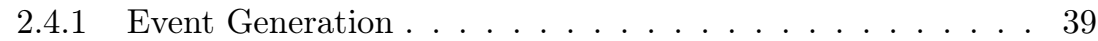

In the $W H \rightarrow \tau \nu b \bar{b}$ Analysis . . . . . . . . . . . . . . . . . . 39

2.4 .2 Detector Simulation . . . . . . . . . . . . . . . . . . . . . 40

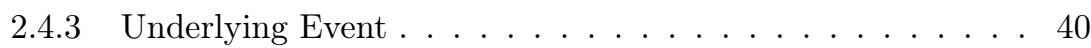

$2.4 .4 \quad$ MC reweighting . . . . . . . . . . . . . . . . . . . . 41

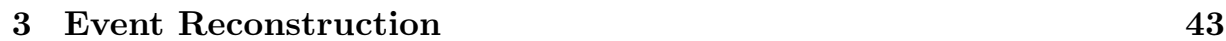

3.1 Tracks . . . . . . . . . . . . . . . . . . . . . . . 43

3.2 Track Quality using CFT Timing . . . . . . . . . . . . . . . . . . . 44

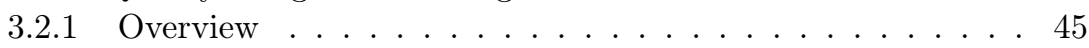

$3.2 .2 \quad$ Basic Chronology of one Fiber Hit . . . . . . . . . . . . . . 45

Expected Time . . . . . . . . . . . . . . . . 47

3.2 .3 Theoretical Model of $t_{\mathrm{f}}$. . . . . . . . . . . . . . . . . . 47

3.2 .4 Data-Derived Corrections . . . . . . . . . . . . . . . . 50

3.2 .5 Track Timing . . . . . . . . . . . . . . . . . . 52

$3.2 .6 \quad$ Fake Track Suppression . . . . . . . . . . . . . . . . . . . 55

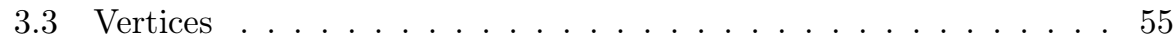

3.4 Muons . . . . . . . . . . . . . . . . . . . 56

3.5 Photons and Electrons . . . . . . . . . . . . . . . . . . . . 56

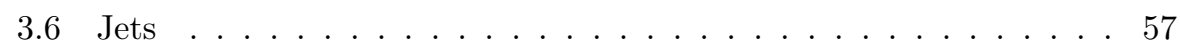

3.6 .1 Calorimeter Noise Reduction . . . . . . . . . . . . . . . 57

3.6 .2 Jet Finding Algorithm . . . . . . . . . . . . . . . . . . . . . 58

Infrared and Collinear Safety . . . . . . . . . . . . . . . . . 58

$3.6 .3 \quad$ Jet Energy Scale . . . . . . . . . . . . . . . . . . . . . . . . . 59

$3.6 .4 \quad$ Jet Energy Resolution . . . . . . . . . . . . . . . . . . . . . 60

3.6 .5 Corrections to Monte Carlo Jets . . . . . . . . . . . . . . . 61

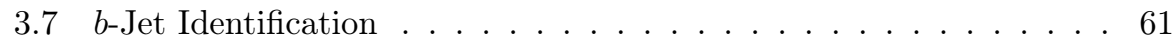

3.8 Tau Identification . . . . . . . . . . . . . . . . . . . . . . . . . . . . 64

3.9 Missing Transverse Energy . . . . . . . . . . . . . . . . . . . . . 67 


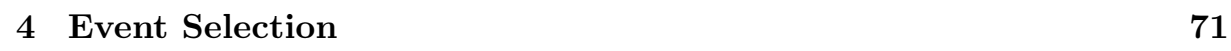

4.1 Data and MC samples . . . . . . . . . . . . . . . . . . 71

4.1 .1 Triggering on Data . . . . . . . . . . . . . . . . 73

4.1 .2 Monte Carlo Datasets . . . . . . . . . . . . . . . . . . 75

Trigger Parameterization . . . . . . . . . . . . . . 78

Reweighting of Boson Transverse Momentum . . . . . . . . 78

4.2 Preselection . . . . . . . . . . . . . . . . . . . . . 78

4.2 .1 Tau Selection . . . . . . . . . . . . . . . . . . . . . . 80

4.2 .2 Jet Selection . . . . . . . . . . . . . . . . . . . . . . . . 80

4.2 .3 Electron and Muon Removal . . . . . . . . . . . . . . . . . 80

4.2 .4 Vertex Requirements . . . . . . . . . . . . . . . . . . . . . . 81

$4.2 .5 \quad$ Multijet Estimation . . . . . . . . . . . . . . . . . . . . 82

4.3 Control Samples . . . . . . . . . . . . . . . . . . . . . . . . . . . . . . . . . 83

4.3 .1 MC Control Sample . . . . . . . . . . . . . . . . . . . 83

4.3 .2 Sideband Region of the MC Sample . . . . . . . . . . . . 85

4.3 .3 Treatment of Bins with Negative Content . . . . . . . . . . 85

4.3 .4 QCD Control Sample . . . . . . . . . . . . . . . . 89

Flat Reweighting . . . . . . . . . . . . . . . . . . . 89

Reweightings in $p_{\mathrm{T}}(\tau)$ and $\eta_{\mathrm{d}}(\tau)$. . . . . . . . . . . . . 89

4.3.5 Summary of Reweightings for the QCD Estimation . . . . . 90

4.4 Signal Sample . . . . . . . . . . . . . . . . . . . . . 90

4.4 .1 Further Selection Cuts . . . . . . . . . . . . . . . . . . . . . 94

4.4 .2 Sideband Region after Further Selection . . . . . . . . . . . 94

4.5 b-Tagging . . . . . . . . . . . . . . . . . . . . 94

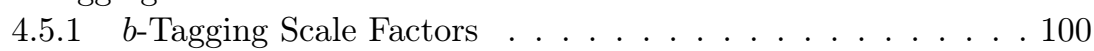

4.5 .2 OneTight $b$-Tag Selection . . . . . . . . . . . . . . . . . . 101

4.5 .3 TwoLoose $b$-Tag Selection . . . . . . . . . . . . . . . . . 102

\begin{tabular}{|llr}
5 & Testing the Higgs Hypothesis & 109
\end{tabular}

5.1 Boosted Decision Trees . . . . . . . . . . . . . . . . . . . . . . . . . 109

5.1 .1 Introduction to Boosted Decision Trees . . . . . . . . . . 110

5.1 .2 Decision Tree Training . . . . . . . . . . . . . . . . . . 111

5.1 .3 Boosting. . . . . . . . . . . . . . . . . 112

5.1 .4 Testing . . . . . . . . . . . . . . . . . . . 112

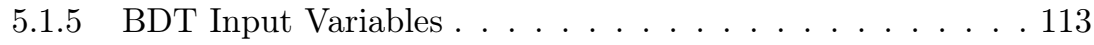

5.1 .6 BDT Output Distributions . . . . . . . . . . . . . . . . 113

5.2 Uncertainties . . . . . . . . . . . . . . . . . . 113

5.2 .1 Flat Systematics . . . . . . . . . . . . . . . . . 117

5.2 .2 Shape Systematics . . . . . . . . . . . . . . . . . . . 118

5.3 Hypothesis Testing . . . . . . . . . . . . . . . . . . . . . . 121

5.3 .1 Negative Log Likelihood Ratio . . . . . . . . . . . . . . . . 121 
5.3 .2 Nuisance Parameters . . . . . . . . . . . . . . . . . . . 122

5.3 .3 Confidence Levels . . . . . . . . . . . . . . . . . . . . 124

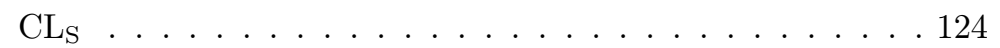

5.3 .4 Limits on the Cross Section . . . . . . . . . . . . . . . . . 124

5.4 Results . . . . . . . . . . . . . . . . . . . . . 126

5.4 .1 Nuisance Parameter Fit . . . . . . . . . . . . . . . . . . . 126

5.4 .2 Confidence Level Limits . . . . . . . . . . . . . . . . . 127

$\begin{array}{lll}6 & \text { Conclusion } & 131\end{array}$

6.1 Discussion on $W H \rightarrow \tau \nu b \vec{b}$. . . . . . . . . . . . . . . . . . . . . . . 131

6.1 .1 Possible Further Improvements . . . . . . . . . . . . . . . . 132

6.2 The DØ Combination . . . . . . . . . . . . . . . 133

$6.2 .1 \quad$ Including the $W H \rightarrow \tau \nu b b$ Channel . . . . . . . . . . . . . . 133

6.3 Tevatron Combination . . . . . . . . . . . . . . . . . . . 134

6.3 .1 Tevatron Prospects . . . . . . . . . . . . . . . . . 136

6.4 Large Hadron Collider Results . . . . . . . . . . . . . . . . . . . . 136

\begin{tabular}{lr}
\hline Bibliography & 141
\end{tabular}

$\begin{array}{ll}\text { Summary } & 151\end{array}$

\begin{tabular}{ll}
\hline Populair-Wetenschappelijke Samenvatting & 157
\end{tabular}

\begin{tabular}{ll}
\hline Acknowledgements & 163
\end{tabular}

\begin{tabular}{ll}
\hline Curriculum Vitae & 165
\end{tabular} 


\section{Introduction}

This thesis describes a search for an elusive particle: the Higgs boson. The search is motivated by the urge to understand the fundamental building blocks of our universe.

Already in the $5^{\text {th }}$ century B.C., the Greek philosophers Leucippus and Democritus formulated the concept of indivisible particles, called atoms. In the beginning of the scientific age, we thought we discovered such particles, so we named them atoms.

Now we know that these atoms are not indivisible at all; they consist of a positively charged nucleus surrounded by an negative electron cloud, where the number of electrons determines most of the chemical properties of the atom. The nucleus itself was found to be comprised of multiple particles: positively charged protons and neutral neutrons. In the 1960s, it was discovered that even the protons and neutrons have constituents, called quarks and gluons.

Quarks and gluons are seen today as infinitesimally small elementary particles, together with the electron and its heavier siblings. Also, the very light neutrinos and the particles responsible for the electromagnetic and weak nuclear forces are thought of as elementary. A very successful theory called the Standard Model was developed, which explained through symmetries how these elementary particles interact. We have to conclude, however, that the electroweak symmetry is broken, due to two observed facts: the particles mediating the weak nuclear force have a non-zero mass and there is a mass difference between particles with different electroweak charge. A popular model that can explain this symmetry breaking is the Higgs mechanism. If this model describes nature, there exists at least one other particle with very specific properties: the Higgs boson.

The search for the Higgs boson takes place at particle colliders, which create an environment where elementary particles have enough energy to form Higgs bosons. The Tevatron, a collider located near Chicago, Illinois, USA, has long been the forefront of Higgs boson searches. Nowadays, however, the Tevatron collider has stopped and the newer and more powerful Large Hadron Collider near Geneva, Switzerland, is taking over. 
As part of an effort by the $\mathrm{D} \varnothing$ collaboration, which uses Tevatron data, we present in this thesis an analysis focused on the process $W H \rightarrow \tau \nu b \bar{b}$. That is, in the analysis we tried to find evidence for the process in which a $W$ boson and a Higgs boson $(H)$ are produced. Specifically, we look at the process where the $W$ decays into particles called a tau and a neutrino and the Higgs boson decays into two $b$-quarks.

First, the theoretical context of the search is explained; the Standard Model will be discussed, including the arguments that lead many to believe there should be a Higgs boson. Its known properties, should it exist, are listed together with its only unknown property: its mass.

If they exist, Higgs bosons are produced at high-energy collisions, albeit with a very low rate. We touch upon the means by which these collisions are generated. Then, the $\mathrm{D} \varnothing$ detector and how it measures the energy and momentum of the collision products is discussed. Not all collisions, also called events, are interesting enough that their data should be stored. How this decision is made is also described, together with how high-energy collisions are simulated.

In Chapter 3, we explain the translation of stored detector information to physical concepts through the identification of particle tracks and different particle types, including those used for the $W H \rightarrow \tau \nu b \bar{b}$ analysis: tau leptons, $b$-jets and missing transverse energy.

Chapter 4 discusses how the events that resemble the $W H \rightarrow \tau \nu b \bar{b}$ process are selected. Also described are the signal and background modeling, including the instrumental background, which is hard to model. This background comes from copiously produced events with only jets in the final state, which are misidentified as having a tau, missing transverse energy and $b$-like jets.

Next, how one can extract most information out of the selected events is examined, with a discussion on the treatment of systematic uncertainties. In this chapter there also is an explanation of how to test the Standard Model Higgs boson hypothesis. Afterwards, the results from the $W H \rightarrow \tau \nu b \bar{b}$ analysis are presented.

Finally, the results are placed in a wider context: that of other DØ searches, the combined Tevatron result - including projections for the future - and Large Hadron Collider results. 


\section{Chapter 1}

\section{Theory}

\subsection{The Standard Model}

The Standard Model (SM) of elementary particle physics is a theory that describes three out of the four currently known fundamental forces of nature, and all known elementary particles. In the 1970s it was constructed to combine the electromagnetic (EM), weak and strong interactions, and all known (anti)particles into one Lagrangian [1 4]. From this Lagrangian, the equations of motion can be derived by the Euler-Lagrange equations.

In order to search for the Higgs boson it is paramount to understand the Standard Model. Conversely, the Higgs boson is an indispensable part of the Standard Model, as is discussed hereafter.

\subsubsection{A Short Introduction}

The particles associated with matter are spin $1 / 2$ fermions, while the forces between them are carried by spin 1 gauge bosons. The EM force is carried by the massless photon, gluons are responsible for the strong force and they too do not have a mass. The weak force has three massive gauge bosons, the $W^{ \pm}$and $Z$ bosons, with a mass of $80.399 \pm 0.023 \mathrm{GeV}$ and $91.1876 \pm 0.0021 \mathrm{GeV}$, respectively. ${ }^{1}$

There are two groups of fermions: quarks, which participate in the strong interaction, and leptons, which do not. Both quarks and leptons are divided into up and down types, where the up-type leptons are called neutrinos and the down-type leptons are the charged leptons. Each of the four fermion types has a specific EM, weak and strong charge, which are listed together with the charges

\footnotetext{
${ }^{1}$ Natural units, defined by $c \equiv \hbar \equiv 1$, are used in this thesis. Therefore, mass and momentum can be denoted in $\mathrm{eV}$.
} 


\begin{tabular}{llccccc}
\hline particle type & symbol $(\mathrm{s})$ & $s$ & $Q$ & $I_{3}^{\mathrm{RH}}$ & $I_{3}^{\mathrm{LH}}$ & color \\
\hline up-type quarks & $u, c, t$ & $1 / 2$ & $+^{2} / 3$ & 0 & $+^{1 / 2}$ & $\mathbf{3}$ \\
down-type quarks & $d, s, b$ & $1 / 2$ & $-1 / 3$ & 0 & $-1 / 2$ & $\mathbf{3}$ \\
neutrinos & $\nu_{1}, \nu_{2}, \nu_{3}$ & $1 / 2$ & 0 & 0 & $+1 / 2$ & $\mathbf{1}$ \\
charged leptons & $e^{-}, \mu^{-}, \tau^{-}$ & $1 / 2$ & -1 & 0 & $-1 / 2$ & $\mathbf{1}$ \\
\hline photon & $\gamma$ & 1 & 0 & 0 & $\mathbf{1}$ \\
gluons & $g$ & 1 & 0 & 0 & $\mathbf{8}$ \\
W-boson & $W^{ \pm}$ & 1 & \pm 1 & \pm 1 & $\mathbf{1}$ \\
Z-boson & $Z$ & 1 & 0 & 0 & $\mathbf{1}$ \\
\hline
\end{tabular}

Table 1.1: SM particles and their quantum numbers: spin $s$, electric charge in unit proton charges $Q$, third component of the isospin $I_{3}$ for particles with right-handed $(\mathrm{RH})$ and left-handed $(\mathrm{LH})$ chirality and color. The listed color value, which belongs to the strong interaction, is not a charge, but is the dimension of the representation of $S U(3) ; \mathbf{1}$ means color neutrality, $\mathbf{3}$ a triplet and $\mathbf{8}$ an octet.

of the gauge bosons in Table1.1. Each fermion type has three different mass eigenstates, so there is a total of twelve fermionic particles. ${ }^{2}$

Note the difference between left-handed and right-handed particles. The handedness, or chirality, is a Lorentz invariant property of a particle. For massless particles the chirality is the same as the helicity, which is defined as the sign of the projection of the momentum vector onto the spin.

Neutrinos were originally assumed massless by the Standard Model. However, the last decades have shown overwhelming evidence that at least two neutrinos have non-zero mass [5]. This is the motivation to treat the neutrinos on equal footing with the other fermions, and to introduce mixing among lepton families. ${ }^{3}$

Antiparticles have the same mass, but opposite charges compared to ordinary particles. Weak isospin is slightly different, because besides the charge, also the handedness flips. The photon, gluons and the $Z$ boson are each their own antiparticle, while the $W^{+}$and $W^{-}$are each other's antiparticle.

The fermions of each type are arranged in generations according to their mass; the first generation has the lightest and the third has the heaviest particle of the same type. The fermion masses are listed in Table 1.2 .

\footnotetext{
${ }^{2}$ The six quarks also have three possible color charges. So it could be argued equally well that there are $3 \times 6+6=24$ fermionic particles.

${ }^{3}$ There are hypotheses where neutrinos (also) have Majorana masses, making the neutrinos their own antiparticles, but there is currently no experimental evidence for this.
} 


\begin{tabular}{lrlrlr}
\hline \multicolumn{2}{c}{ generation I } & \multicolumn{2}{c}{ generation II } & \multicolumn{2}{c}{ generation III } \\
fermion & mass $(\mathrm{MeV})$ & fermion & mass $(\mathrm{MeV})$ & fermion & mass $(\mathrm{MeV})$ \\
\hline$u$ & $1.7-3.3$ & $c$ & $1.27 \cdot 10^{3}$ & $t$ & $172 \cdot 10^{3}$ \\
$d$ & $4.1-5.8$ & $s$ & 101 & $b$ & $4.19 \cdot 10^{3}$ \\
$e^{-}$ & 0.511 & $\mu^{-}$ & 106 & $\tau^{-}$ & 1777 \\
\hline
\end{tabular}

Table 1.2: fermion masses as reported by the Particle Data Group [6]. The neutrino masses are yet unknown, although the differences between the mass squared are known: $\left|m_{\nu_{1}}^{2}-m_{\nu_{2}}^{2}\right|=(76 \pm 2) \cdot 10^{-6} \mathrm{eV}^{2}$ and $\left|m_{\nu_{1}}^{2}-m_{\nu_{3}}^{2}\right|=(2.43 \pm 0.13) \cdot 10^{-3} \mathrm{eV}^{2}$.

\subsubsection{Quantum Field Theory}

The SM is described by quantum field theory (QFT) with a Lagrangian density. The elementary excitations of the quantized fields are interpreted as particles. Therefore, terms in the Lagrangian can be visualized at the particle level; terms with two fields describe the propagation of particles, while terms with three or four fields describe interactions of the corresponding particles at a single spacetime point, also called a vertex.

\section{Feynman Diagrams}

QFT processes can be described pictorially by Feynman diagrams [7. Examples of them can be found in Figures 1.1, 1.2, 1.5 and 1.7. The open-ended lines on the left side represent initial-state particles. Their interaction with other particles happens at the vertices and the final-state particles are indicated by the openended lines on the right side of the diagram. The particle propagation between vertices is also represented by lines. The line shapes indicate the spin of the particle, with dashed lines for spin-0 bosons and wiggly lines for spin-1 bosons, with the exception of gluons, which are represented by curly lines. Fermions have right-pointing arrowed lines, while antifermions have arrows pointing left.

\section{Local Gauge Theory}

The Lagrangian exhibits continuous symmetries, corresponding to the interactions. Although these symmetries only hold globally, observers can choose different gauges locally. A local gauge theory is then needed to account for the position-dependent choice.

Quantum electrodynamics (QED) is a $U(1)$ symmetric example of such a local gauge theory. To derive it, we need to look at the relativistically covariant 
equation of motion of a spin $\frac{1}{2}$ particle, described by the Dirac equation,

$$
\left(i \gamma^{\mu} \partial_{\mu}-m\right) \psi(x)=0
$$

where $\psi(x)$ is the fermion field, $m$ is the particle mass and $\gamma^{\mu}$ are the Dirac matrices, satisfying $\left\{\gamma^{\mu}, \gamma^{\nu}\right\}=2 g^{\mu \nu}$, with $g^{\mu \nu}$ the metric tensor.

The Lagrangian density associated with this equation of motion is ${ }^{4}$

$$
\mathcal{L}(x)=\bar{\psi}(x)\left(i \gamma^{\mu} \partial_{\mu}-m\right) \psi(x),
$$

which has a global $U(1)$ symmetry, given by the transformation $\psi(x) \rightarrow e^{i \theta} \psi(x)$. If we now demand that this symmetry holds locally, namely for $\theta=\theta(x)$, the Lagrangian can only be invariant if the derivative $\partial_{\mu}$ is replaced by the covariant derivative $D_{\mu}=\partial_{\mu}+i e A_{\mu}(x)$, where $e$ is the charge and $A_{\mu}$ is a new vector field. The new Lagrangian transforms now as

$$
\begin{aligned}
\mathcal{L} & \rightarrow \bar{\psi} e^{-i \theta}\left(i \gamma^{\mu}\left(\partial_{\mu}+i e A_{\mu}^{\prime}\right)-m\right) e^{i \theta} \psi \\
& =\bar{\psi}\left(i \gamma^{\mu}\left(\partial_{\mu}+i\left(\partial_{\mu} \theta\right)+i e A_{\mu}^{\prime}\right)-m\right) \psi,
\end{aligned}
$$

and we demand the Lagrangian to be invariant, so the gauge field transforms as

$$
A_{\mu} \rightarrow A_{\mu}^{\prime}=A_{\mu}-\frac{1}{e}\left(\partial_{\mu} \theta\right)
$$

If we include the dynamics of the gauge field, we get

$$
\mathcal{L}=\bar{\psi}\left(i \gamma^{\mu} D_{\mu}-m\right) \psi-\frac{1}{4} F_{\mu \nu} F^{\mu \nu},
$$

with $F_{\mu \nu}=\partial_{\mu} A_{\nu}-\partial_{\nu} A_{\mu}$ the field tensor. The gauge boson $A$ must be massless because mass terms, which take the form $m_{A}^{2} A_{\mu} A^{\mu}$, would break the invariance of the Lagrangian.

\section{Non-Abelian Gauge Theories}

In the case of the strong and the weak interaction, the Lagrangian is constructed in a similar way, but now with a non-Abelian symmetry group. The symmetries we observe in nature are Lie groups, which transform the fermion fields as

$$
\psi \rightarrow e^{i \theta^{a} t^{a}} \psi
$$

where $t^{a}$ are the generators of the Lie group.

\footnotetext{
${ }^{4}$ The Lagrangian is always Hermitian, so when a non-Hermitian Lagrangian appears in this thesis, '+ Hermitian conjugate' is implied.
} 
The Lagrangian for non-Abelian groups is still given by Equation (1.5), but now with

$$
\begin{aligned}
D_{\mu} & =\partial_{\mu}+i g t^{a} A_{\mu}^{a} \text { and } \\
F_{\mu \nu}^{a} & =\partial_{\mu} A_{\nu}^{a}-\partial_{\nu} A_{\mu}^{a}+g f^{a b c} A_{\mu}^{b} A_{\nu}^{c},
\end{aligned}
$$

with $f^{a b c}$ the structure constants of the symmetry group and $g$ the coupling constant of the associated force.

The strong force is described by Quantum Chromo Dynamics (QCD), which has $S U(3)$ symmetry. Therefore it has a charge triplet, the colors, and $3^{2}-1=8$ independent $t^{a}$ s corresponding to eight gluons.

The weak interaction is another example of a non-Abelian gauge theory and can be unified with electrodynamics in the $S U(2)_{L} \times U(1)_{Y}$ symmetric electroweak theory. ${ }^{5}$ The covariant derivative of this theory is

$$
D_{\mu}=\partial_{\mu}-\frac{1}{2} i g t^{a} A_{\mu}^{a}-\frac{1}{2} i g^{\prime} Y B_{\mu},
$$

with $g$ and $g^{\prime}$ the coupling constants, $Y=2\left(Q-I_{3}\right)$, the hypercharge where $I_{3}$ is the third component of the weak isospin and $Q$ the electric charge. The Lie group generators $t^{a}$ are the Pauli matrices for left-handed fermions, which are isospin doublets, and zero for right-handed fermions, the isospin singlets.

The $A^{a}$ and $B$ bosons still have to be massless if $S U(2)_{L} \times U(1)_{Y}$ is a true symmetry.

\section{Spontaneous Symmetry Breaking}

It is possible to add mass terms by hand to the Lagrangian and break the symmetry explicitly, but doing so would lead to a non-renormalizable theory. We know, however, that the $W$ and $Z$ bosons do have mass. To solve this problem the Glashow-Weinberg-Salam model was introduced [1]3, 8, 13.

Suppose there is a complex-valued scalar field $\phi$ which transforms as a doublet under isospin and has $Y=1$. If we add all possible $\phi$ terms that preserve renormalizability and are gauge invariant to the Lagrangian, we get

$$
\begin{aligned}
\mathcal{L}_{\text {Higgs }} & =\left(D_{\mu} \phi\right)^{\dagger} D^{\mu} \phi-V(\phi), \text { where } \\
V(\phi) & =\mu^{2}|\phi|^{2}+\lambda|\phi|^{4},
\end{aligned}
$$

with $D_{\mu}$ defined by Equation (1.8) and $\mu, \lambda$ free parameters. The potential should be bounded from below, which means $\lambda>0$. Now there are two possibilities:

$\mu^{2}>0$, which would mean a $\phi_{0}=0$ ground state with no symmetry breaking;

\footnotetext{
${ }^{5} U(1)_{Y}$ only differs from $U(1)_{Q}$ of QED by replacing the electric charge $Q$ with the hypercharge $Y$.
} 
$\mu^{2}<0$, where the potential is given by the famous 'mexican hat' potential with the ground state given by $\left|\phi_{0}\right|^{2}=\frac{-\mu^{2}}{2 \lambda} \equiv \frac{1}{2} v^{2}$.

Because we observe non-zero gauge boson masses, $\mu^{2}<0$ is likely realized in nature. Using rotational freedom we can choose the ground state to be

$$
\langle\phi\rangle=\frac{1}{\sqrt{2}}\left(\begin{array}{l}
0 \\
v
\end{array}\right) .
$$

We can take linear combinations of the gauge fields of Equation 1.8 to construct another orthogonal basis:

$$
\begin{aligned}
A_{\mu} & =\frac{1}{\sqrt{g^{2}+g^{\prime 2}}}\left(g^{\prime} A_{\mu}^{3}+g B_{\mu}\right), \\
Z_{\mu} & =\frac{1}{\sqrt{g^{2}+g^{\prime 2}}}\left(g A_{\mu}^{3}-g^{\prime} B_{\mu}\right) \text { and } \\
W_{\mu}^{ \pm} & =\frac{1}{\sqrt{2}}\left(A_{\mu}^{1} \mp i A_{\mu}^{2}\right),
\end{aligned}
$$

so the kinetic terms in the Lagrangian of Equation 1.9 become

$$
\mathcal{L}_{\text {mass }}=m_{W}^{2} W_{\mu}^{+} W^{-\mu}+\frac{1}{2} m_{Z}^{2} Z_{\mu} Z^{\mu},
$$

with $m_{W}=\frac{1}{2} v g$ and $m_{Z}=\frac{1}{2} v \sqrt{g^{2}+g^{\prime 2}}$. The $W$ and $Z$ bosons now have a non-zero mass, while the photon field $A$ is still massless.

With this model, the electroweak symmetry is not broken explicitly, but by the asymmetry of the ground state. This is called spontaneous symmetry breaking.

If there is a scalar doublet field, there are other terms that can be added to the Lagrangian: the Yukawa couplings. For a fermion $f$ this coupling is given by

$$
\mathcal{L}_{f}=-\lambda_{f^{d}} \bar{F}_{L} \phi f_{R}^{d}-\lambda_{f^{d}} \bar{f}_{R}^{d} \phi^{\dagger} F_{L}-\lambda_{f^{u}} \bar{F}_{L}^{a} \epsilon^{a b} \phi^{b} f_{R}^{u}-\lambda_{f^{u}} \bar{f}_{R}^{u} \phi^{\dagger a} \epsilon^{a b} F_{L}^{b},
$$

where the $\lambda_{\mathrm{s}}$ are arbitrary coupling constants, and $f^{u}$ and $f^{d}$ are the fermion fields for up-type fermions $\left(u, c, t, \nu_{1}, \nu_{2}\right.$ and $\left.\nu_{3}\right)$ and down-type fermions $(d, s$, $b, e, \mu$ and $\tau$ ), which are combined in the doublets

$$
F_{L}=\left(\begin{array}{c}
f_{L}^{u} \\
f_{L}^{d}
\end{array}\right)
$$

If field $\phi$ is at its vacuum expectation value, $\phi=\langle\phi\rangle$, Equation 1.13 becomes

$$
\mathcal{L}_{f}=-m_{f^{d}}\left(\bar{f}_{L}^{d} f_{R}^{d}+\bar{f}_{R}^{d} f_{L}^{d}\right)-m_{f^{u}}\left(\bar{f}_{L}^{u} f_{R}^{u}+\bar{f}_{R}^{u} f_{L}^{u}\right),
$$

with $m_{f}=\lambda_{f} \frac{v}{\sqrt{2}}$ the fermion masses. So the vacuum expectation value of the scalar field can also provide for the fermion masses. 
Actually, in Equation 1.13 more general coupling constants $\lambda_{f}^{i j}$ are allowed, where fermions $i$ and $j$ of different mass but same quantum numbers are mixed. By a global, unitary transformation of the fields, $\lambda$ can be diagonalized. No terms in the Lagrangian are affected, except terms like $\bar{f}_{L}^{u, i} f_{R}^{d, i} W^{+} \rightarrow \bar{f}_{L}^{u, i} V^{i j} f_{R}^{d, j} W^{+}$.

It is conventional to interpret this for the quark sector as the up-types now coupling to rotated versions of the down-types, $f^{d, i} \rightarrow f^{d^{\prime}, i}=V_{\mathrm{CKM}}^{i j} f^{d, j}$. The rotated down type quarks are called $d^{\prime}, s^{\prime}$ and $b^{\prime}$, and $V_{\text {CKM }}$ is the CabibboKobyashi-Maskawa (CKM) matrix.

In the lepton case the neutrinos $\nu_{1}, \nu_{2}$ and $\nu_{3}$ are rotated into $\nu_{e}, \nu_{\mu}$ and $\nu_{\tau}$ by the Pontecorvo-Maki-Nakagawa-Sakata (PMNS) matrix. The absolute values of the elements of these mixing matrices are given by [6]:

$$
\left(\begin{array}{ccc}
0.97 & 0.23 & 0.003 \\
0.23 & 0.97 & 0.04 \\
0.008 & 0.04 & 0.999
\end{array}\right)_{\mathrm{CKM}} \text { and } \quad\left(\begin{array}{ccc}
0.73-0.89 & 0.45-0.66 & <0.24 \\
0.23-0.66 & 0.24-0.75 & 0.52-0.87 \\
0.06-0.57 & 0.40-0.82 & 0.48-0.85
\end{array}\right)_{\mathrm{PMNS}} \text {. }
$$

Note that the CKM matrix is very close to diagonal. A priori the SM does not require the quarks to mix this little and to interact mainly with their generational partner. It is also not known why the lepton mixing matrix is so different from the quark one.

\subsection{The Higgs Boson}

The scalar field $\phi$ can have variations around the ground state, parameterized by the Higgs field $H(x)$. We can write $\phi$ as

$$
\phi(x)=\frac{1}{\sqrt{2}}\left(\begin{array}{c}
0 \\
v+H(x)
\end{array}\right) .
$$

The Higgs potential now becomes

$$
\mathcal{L}_{V(\phi)}=\frac{m_{H}^{2} v^{2}}{8}-\frac{m_{H}^{2}}{2} H^{2}-\frac{m_{H}^{2}}{2 v} H^{3}-\frac{m_{H}^{2}}{8 v^{2}} H^{4},
$$

which shows there is a particle, the Higgs boson, with mass $m_{H}=\sqrt{2 \lambda v^{2}}$ and self interactions with three or four particles in a vertex.

The variation on the field $\phi$ has to be added everywhere in the Lagrangian. This results in terms describing interactions of the Higgs boson with the fermions and massive gauge bosons:

$$
\begin{aligned}
\mathcal{L}_{H}= & -\frac{m_{f}}{v} H\left(\bar{f}_{L} f_{R}+\bar{f}_{R} f_{L}\right)+2 \frac{m_{W}^{2}}{v} H W_{\mu}^{+} W^{-\mu}+\frac{m_{Z}^{2}}{v} H Z_{\mu} Z^{\mu} \\
& +\frac{m_{W}^{2}}{v^{2}} H^{2} W_{\mu}^{+} W^{-\mu}+\frac{m_{Z}^{2}}{2 v^{2}} H^{2} Z_{\mu} Z^{\mu} .
\end{aligned}
$$




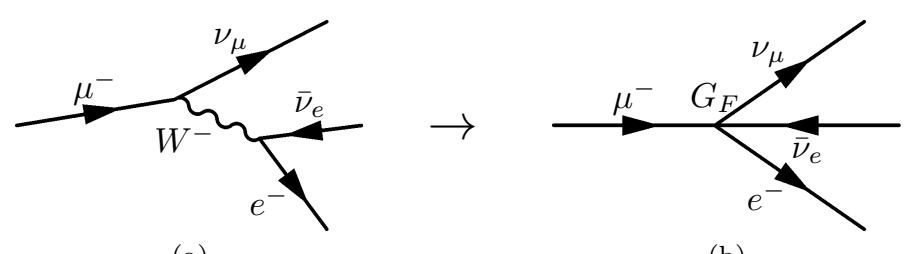

(a)

(b)

Figure 1.1: the muon decay process with a $W$ exchange (a) becomes an effective four-fermion coupling (b) at low energies.

Note that the coupling constant of the Higgs boson with a particle is proportional to the particle mass, because coupling constants appear linearly in the Lagrangian for fermions and quadratically for gauge bosons.

It is also possible to have two or more scalar fields, resulting in multiple physical Higgs bosons. This is however beyond the scope of this thesis.

\subsubsection{Known Properties}

In adding the scalar field, we introduced two unknown parameters. The vacuum expectation value produces the $W$ mass, which can be determined very accurately by measuring muon decay. This process is historically described by the Fermi coupling constant $G_{F}$, and, as can be seen in Figure 1.1, is a low energy approximation of a $W$ exchange. Now we know

$$
v=\frac{2 g}{m_{W}}=\sqrt{\frac{1}{\sqrt{2} G_{F}}} \approx 246 \mathrm{GeV},
$$

and are left with one unknown parameter, the Higgs mass.

\section{Unitarity Bound}

Another way to see that massive gauge bosons are a problem, is by looking at the unitarity bound. We know the transition of one state into another can have maximally a $100 \%$ probability. This translates into the requirement that matrix elements $\mathcal{M}$ of the $S$-matrix can grow with energy $E$ at most with $E^{4-N}$, where $N$ is the number of external (either incoming or outgoing) vector bosons.

For example, we can examine the situation where two oppositely charged and longitudinally polarized $W$ bosons interact and form two longitudinally polarized $Z$ bosons, or in short $W_{L}^{+} W_{L}^{-} \rightarrow Z_{L} Z_{L}$. At lowest order in $g$ we have only tree level processes, which can be visualized by the Feynman diagrams shown in Figure 1.2 . 

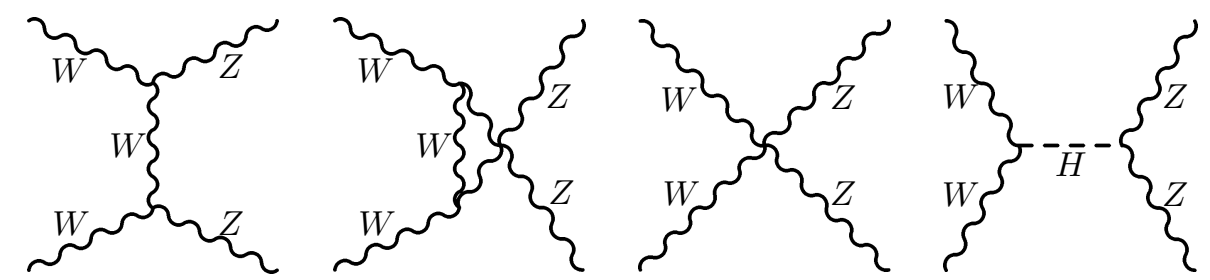

Figure 1.2: Feynman diagrams contributing at tree level to $W^{+} W^{-} \rightarrow Z Z$.

Without a Higgs boson the last diagram is not present and $\mathcal{M}$ becomes

$$
\mathcal{M}_{\text {no Higgs }}=\frac{-i e^{2} m_{Z}^{2} E^{2}}{4 m_{W}^{2}\left(m_{Z}^{2}-m_{W}^{2}\right)}+\mathcal{O}\left(E^{0}\right),
$$

which will break unitarity for sufficiently large energy. If there is a Higgs boson, having the vector boson couplings given by the Lagrangian described in Section 1.1.2, the last diagram will cancel the $E^{2}$ term, which only leaves

$$
\mathcal{M}_{\text {with Higgs }}=\mathcal{O}\left(E^{0}\right) \text {. }
$$

In Figure 1.3 [14 the two amplitudes are plotted against energy. The 'bump' is the resonance around the Higgs mass, $200 \mathrm{GeV}$ in this graph.

This is another argument why (a scalar particle similar to) a Higgs boson should exist. The same bound also provides a constraint on its mass, by requiring that the process including a Higgs boson too has to remain within the unitarity bound for all energies. If all vector boson scatterings are taken into account, the limit on the Higgs mass by the unitarity bound is [15]:

$$
m_{H} \lesssim \sqrt{\frac{16 \pi}{3}} v \approx 1 \mathrm{TeV}
$$

\section{Running Coupling Constraints}

Coupling constants depend on the energy with which they are probed. In quantum field theory additional particles can form off the mass shell (so $p^{2} \neq m^{2}$, with $p$ the four-momentum). If more energy becomes available, these higher order processes become more relevant. ${ }^{6}$ Then, a coupling at tree level is replaced by an effective coupling, which includes all the additional terms. This 'running' of the coupling constants with energy is described by renormalization group equations.

\footnotetext{
${ }^{6}$ Actually, in QCD the effect is opposite because of antiscreening.
} 


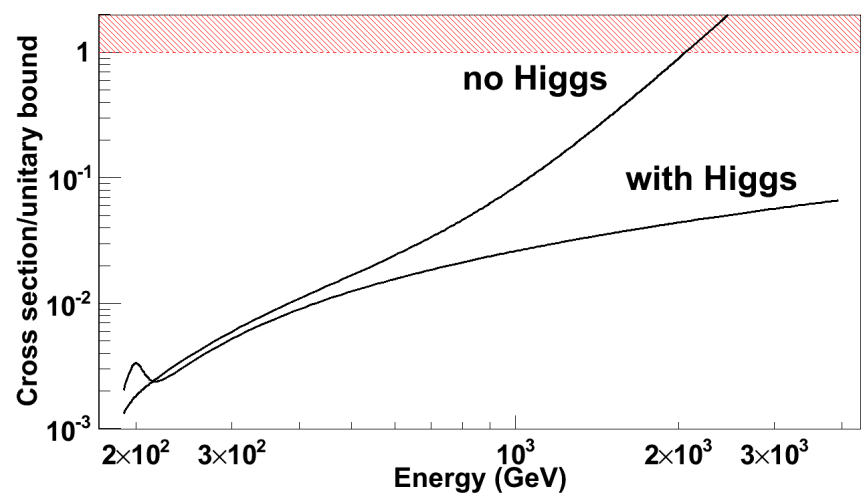

Figure 1.3: the cross section over the unitarity bound as a function of center-ofmass energy for a scenario with a $200 \mathrm{GeV}$ Higgs boson and without one as calculated by the Camorra algorithm [14. The shaded region is not allowed by unitarity.

For the Higgs self-coupling constant $\lambda$, the renormalization group equation

$$
16 \pi^{2} \frac{\mathrm{d} \lambda}{\mathrm{d} \ln (Q)}=24 \lambda^{2}-6 \lambda_{t}^{4}+\ldots
$$

holds [16], with $Q$ the energy scale and $\lambda_{t}$ the coupling to the top quark, as described in Equation (1.13).

The Higgs quartic coupling has to be in a reasonable range. For example $\lambda$ could grow to infinity for an energy called the Landau pole $\Lambda_{\mathrm{L}}$. If we demand the coupling to be finite, either new processes could stop the running from diverging, or $\lambda$ at any lower energy scale has to be zero. This is called the triviality bound, because for $\lambda=0$ we could not have a vacuum expectation value. Whichever of the other two scenarios happens, at or before $\Lambda_{\mathrm{L}}$ we should see new physics: infinite couplings or new processes.

On the other hand, if $\lambda$ is below zero for a certain energy, the potential is not bounded from below anymore. In that case the vacuum is not stable, so we call this the vacuum stability limit $\Lambda_{\mathrm{V}}$. It can also happen that new processes make sure $\lambda$ stays positive. Either way, at or before $\Lambda_{\mathrm{V}}$ we see non-SM physics.

To find the Landau pole $\Lambda_{\mathrm{L}}$ we take $\lambda \gg \lambda_{t}^{2}$. We can now solve Equation (1.24) by

$$
\lambda(Q)=\frac{\lambda(\mu)}{1-\frac{3}{2 \pi^{2}} \lambda(\mu) \ln \left(\frac{Q}{\mu}\right)},
$$

where the integration constant $\mu$ is another energy scale, for example where the 
Higgs mass is defined. Without new physics occurring we need at least

$$
1>\frac{3}{2 \pi^{2}} \lambda(\mu) \ln \left(\frac{\Lambda_{\mathrm{L}}}{\mu}\right) \Longleftrightarrow m_{H}(\mu)<\frac{2 \pi v}{\sqrt{3}} \sqrt{\ln \left(\frac{\Lambda_{\mathrm{L}}}{\mu}\right)^{-1}},
$$

with the $\Lambda_{\mathrm{L}}$ the energy scale where $\lambda$ goes to infinity.

From $\lambda \ll \lambda_{t}$ we can get the vacuum stability limit. Up to leading order the top mass is constant in energy, so the renormalization group equation becomes

$$
\lambda(Q)=\lambda(\mu)-\frac{3 m_{t}^{4}}{2 \pi^{2} v^{4}} \ln \left(\frac{Q}{\mu}\right) .
$$

There will be new physics at scale $\Lambda_{\mathrm{V}}$ before $\lambda$ becomes negative, so

$$
m_{H}(\mu)>\frac{m_{t}^{2}}{\pi v} \sqrt{3 \ln \left(\frac{\Lambda_{\mathrm{V}}}{\mu}\right)} .
$$

Combining the two limits, we get for the scale $\Lambda$ where there has to be new physics:

$$
\frac{m_{t}^{2}}{\pi v} \sqrt{3 \ln \left(\frac{\Lambda}{\mu}\right)}<m_{H}(\mu)<\frac{2 \pi v}{\sqrt{3}} \sqrt{\ln \left(\frac{\Lambda}{\mu}\right)^{-1}} .
$$

The energy scales at which new physics is to be found for a given Higgs mass have been plotted in Figure 1.4 [17, which also includes higher order corrections to the above calculations. Conversely, the plot describes theoretically allowed Higgs masses if up to a certain energy range no new physics in addition to the $\mathrm{SM}$ has been discovered. The figure also shows the Tevatron exclusion region, a result that will be discussed in Chapter6.

\section{Indirect Measurements}

Higher orders in perturbation theory provide corrections to the ratio of the $W$ and $Z$ masses. The top-quark mass enters this ratio quadratically, while the Higgs mass only has a logarithmic contribution. Collider experiments determined $m_{Z}=91.1875 \pm 0.0021 \mathrm{GeV}, m_{W}=80.399 \pm 0.023 \mathrm{GeV}$ and $m_{t}=173.2 \pm 0.9 \mathrm{GeV}$. With these and other electroweak data the Higgs mass can be fitted to be

$$
m_{H}=95_{-24}^{+30} \mathrm{GeV}
$$

and with $95 \%$ confidence level (CL) the fit yields $m_{H}<169 \mathrm{GeV}$ [18]. 


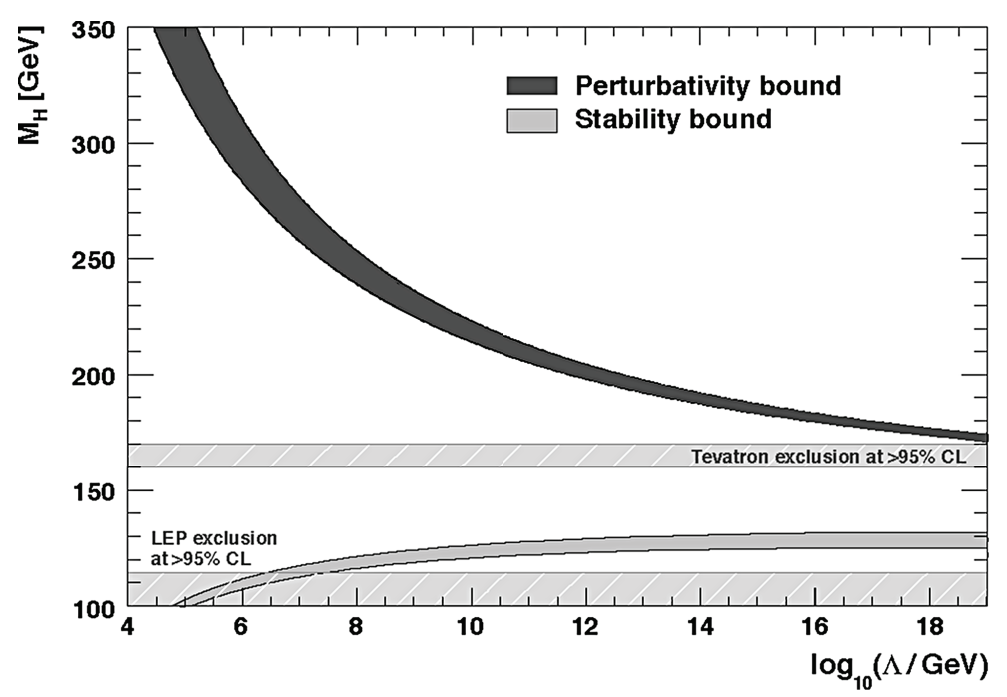

Figure 1.4: the energy scale where we will see new physics, depending on the Higgs mass (adapted from [17]). The bounds have a width given by uncertainties in SM parameters, like the top-quark mass. The width of the triviality (or perturbativity) bound also includes different possible contributions from higher-order perturbative corrections.

\section{Direct Searches}

At the large electron-positron (LEP) collider, the main production channel for Higgs bosons is so-called Higgs-strahlung. The electron and positron form a $Z$ boson, from which a Higgs boson is radiated, as can be seen in Figure 1.5

LEP produced electron-positron collisions with center-of-mass energies up to $209 \mathrm{GeV}$. Because no observation of a Higgs boson was made, the working group for Higgs boson searches published their 95\% CL exclusion of a SM Higgs boson lighter than $114.4 \mathrm{GeV}$ [19]. In Figure 1.6 the result from the fit to the precision measurements is shown, along with the exclusion regions from the direct searches.

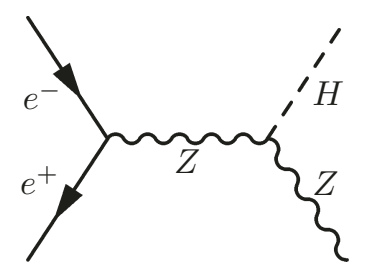

Figure 1.5: leading diagram of $e^{+} e^{-} \rightarrow Z H$. 


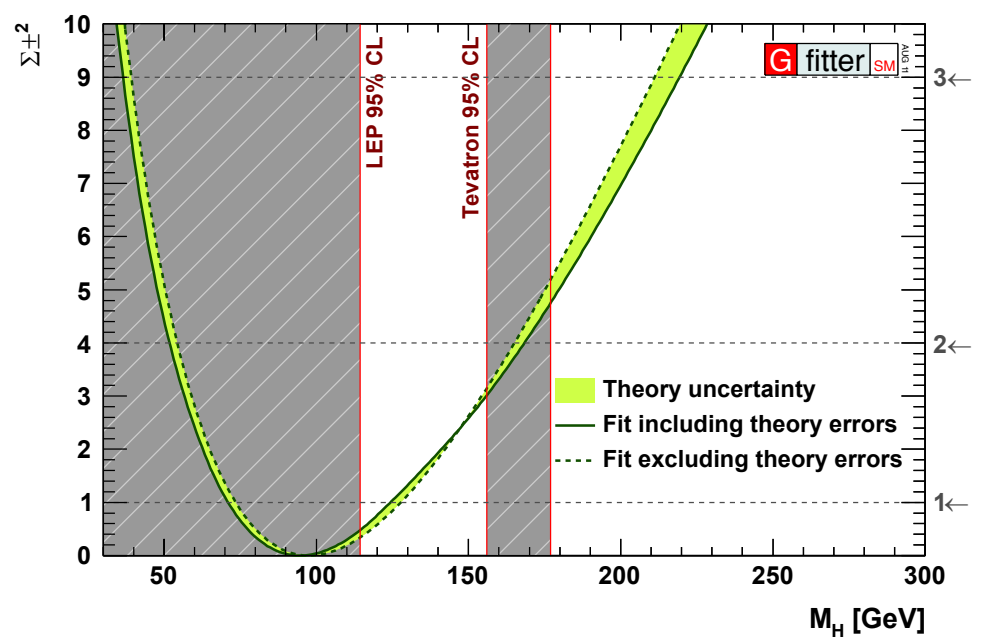

Figure 1.6: the difference in $\chi^{2}$ from the SM fit as a function of a fixed Higgs mass in the fit. The shaded regions are excluded at 95\% CL by LEP and Tevatron experiments [18].

\subsubsection{At the Tevatron}

The Tevatron is a proton-antiproton $(p \bar{p})$ collider at $1.96 \mathrm{TeV}$ average center-ofmass energy. At this energy there are two leading processes for Higgs production, as shown in Figure 1.7, gluon fusion through a top loop and Higgs-strahlung.

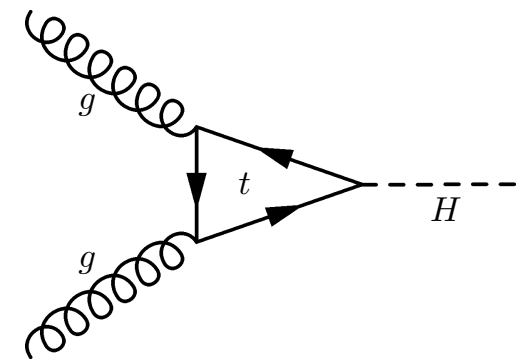

(a) gluon fusion

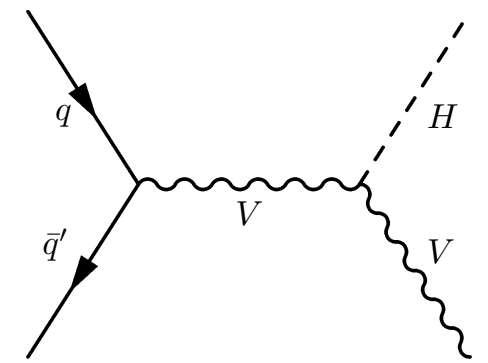

(b) associated vector boson production

Figure 1.7: main contributions to Higgs boson production at the Tevatron. The vector boson $V$ can be either a $Z$ or a $W^{ \pm}$boson. 


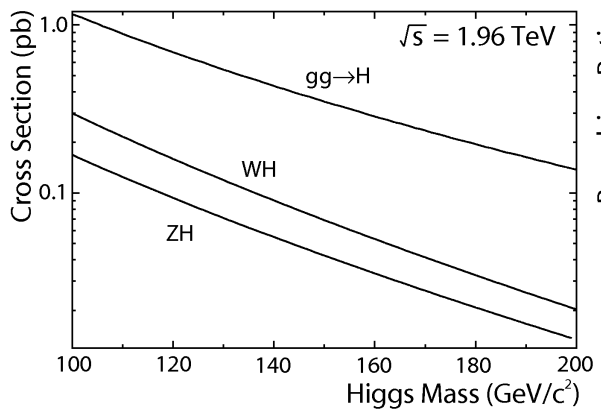

(a) Higgs boson production cross section

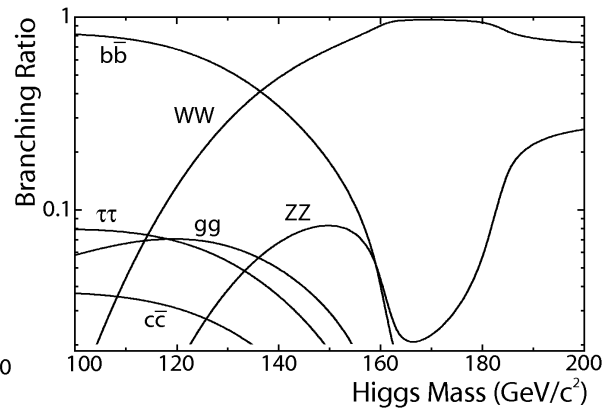

(b) Higgs boson branching ratio

Figure 1.8: most important Higgs boson production (a) and decay modes (b) at the Tevatron [20, 21].

Higgs-strahlung, also called associated vector boson production, suffers from the small couplings of the weak force. This makes gluon fusion about four times more likely to occur, as can be seen in Figure 1.8(a). The total production cross section is about 1.8 to $0.21 \mathrm{pb}$ in the Higgs mass range 100 to $200 \mathrm{GeV}$ [20].

The Higgs boson coupling to other particles is proportional to their mass, as shown in Section 1.1.2. The Higgs mass should also be large enough to produce the decay products not too far off shell. These are the defining ingredients for the relative strength of the decay channels, plotted in Figure 1.8(b) There are roughly two regimes:

$m_{H} \lesssim 135 \mathrm{GeV}$, where the Higgs boson mainly decays into a $b \bar{b}$ pair. At a hadron collider such as the Tevatron, the irreducible background of $b \bar{b}$ is far too high. Therefore it is only possible to trigger on the associated vector boson production with the $W$ or $Z$ decaying leptonically;

$m_{H} \gtrsim 135 \mathrm{GeV}$. Here the Higgs boson decays dominantly into two $W$ bosons. Gluon fusion can now be used as a production mechanism if at least one of the $W$ s decays into leptons.

This thesis will focus on a low Higgs mass scenario. There are six main decay channels for low mass Higgs bosons at the Tevatron. They are listed with their cross section times branching ratio in Table 1.3 . Because of its rapid decay, the tau is treated differently from other charged leptons. Although detector effects can alter the relative importance of the channels, it is clear $W H \rightarrow \tau \nu b \bar{b}^{7}$ can have a non-negligible contribution.

\footnotetext{
${ }^{7} W H \rightarrow \tau \nu b \bar{b}$ is shorthand for $W^{+} H \rightarrow \tau^{+} \nu_{\tau} b \bar{b}$ and $W^{-} H \rightarrow \tau^{-} \bar{\nu}_{\tau} b \bar{b}$. Also in other production and decay modes a similar shorthand notation is used.
} 


\begin{tabular}{crr}
\hline & \multicolumn{2}{c}{ cross section $\times$ branching ratio $(\mathrm{fb})$} \\
process & $m_{H}=115 \mathrm{GeV}$ & $m_{H}=130 \mathrm{GeV}$ \\
\hline$H \rightarrow W W$ & 35 & 95 \\
$Z H \rightarrow \nu \nu b \bar{b}$ & 15 & 8 \\
$Z H \rightarrow \ell \ell b \bar{b}$ & 5 & 3 \\
$Z H \rightarrow \tau \tau j j$ & 6 & 3 \\
$W H \rightarrow \ell \nu b \bar{b}$ & 33 & 18 \\
$W H \rightarrow \tau \nu_{\tau} b \bar{b}$ & 17 & 9 \\
\hline
\end{tabular}

Table 1.3: cross section times branching ratio for different Higgs boson decays. Of the two $W$ s at least one decays into leptons. The lepton $\ell$ here can either be an electron or a muon, and $j$ is any kind of jet, so gluons, quarks or taus. 


\section{Chapter 2}

\section{Experimental Setup}

The high-energy collisions used in this thesis were produced at the Fermi National Accelerator Laboratory (FNAL or Fermilab). The highest-energy accelerator at Fermilab was the Tevatron, a ring shaped synchrotron that could collide protons and antiprotons at $1.96 \mathrm{TeV}$ center-of-mass energy.

Between 1989 and 1996 the Tevatron Run I took place, in which the centerof-mass energy was $1.8 \mathrm{TeV}$. At first, the only experiment that was supposed to be built for Run I was the Collider Detector at Fermilab (CDF). In 1992 the D $\varnothing$ detector was also completed to provide an independent check of CDF's results. Both experiments independently discovered the top quark in 1995 [22].

Run II took place from 2000 until 2011. Several upgrades for Run II have improved the accelerator and detector performances. One of the main accelerator upgrades was the replacement of the Main Ring, which shared its tunnel with the Tevatron, by the Main Injector in its own tunnel. Also, the peak instantaneous luminosity delivered by the Tevatron increased from $10^{31}$ to $3 \cdot 10^{32} \mathrm{~cm}^{-2} \mathrm{~s}^{-1}$ and the time between the crossing of (anti)proton bunches decreased from $3.6 \mu \mathrm{s}$ to 396 ns. The D $\varnothing$ detector was upgraded to use this improvement and to reduce radiation damage. The central tracker was replaced and a solenoid magnet was added, along with preshower detectors and an enhanced muon system.

Because only Run II data is used for the analysis in this thesis, the description of the experimental setup will be that of Run II.

By the end of Run II, on September $31^{\text {st }} 2011$, the Tevatron delivered a total integrated luminosity of $11.9 \mathrm{fb}^{-1}$. DØ recorded $10.7 \mathrm{fb}^{-1}$ of that, with losses coming from detector downtime in the beginning or end of a store, and from temporary (sub)detector problems that prohibited data taking. 


\begin{tabular}{lllrl}
\hline step & machine & product & energy & destination \\
\hline 1 & Pre-accelerator & $\mathrm{H}^{-}$ & $750 \mathrm{keV}$ & Linac \\
2 & Linac & $\mathrm{H}^{-}$ & $400 \mathrm{MeV}$ & Booster \\
3 & Booster & $p$ & $8 \mathrm{GeV}$ & Main Injector, steps $4 \& 7$ \\
4 & Main Injector & $p$ & $120 \mathrm{GeV}$ & Pbar source \\
5 & Pbar Source & $\bar{p}$ & $8 \mathrm{GeV}$ & Recycler \\
6 & Recycler & $\bar{p}$ & $8 \mathrm{GeV}$ & Main Injector \\
7 & Main Injector & $p, \bar{p}$ & $150 \mathrm{GeV}$ & Tevatron \\
8 & Tevatron & $p, \bar{p}$ & $980 \mathrm{GeV}$ & collisions \& beam dump \\
\hline
\end{tabular}

Table 2.1: the acceleration chain leading up to $980 \mathrm{GeV}$ (anti)protons. The energy quoted is the kinetic energy of the particles at the end of the acceleration stage.

\subsection{Proton-Antiproton Collisions}

To get proton-antiproton collisions with a center-of-mass energy of $1.96 \mathrm{TeV}$, antiprotons are made and accelerated, together with the protons, to $980 \mathrm{GeV}$.

The acceleration chain of the (anti)protons is summarized in Table 2.1, and Figure 2.1 shows the layout of the accelerators. The different stages, the Proton and Antiproton Source, the Main Injector, Recycler and the Tevatron, are described below. More information on Fermilab's accelerators can be found in [23].

\subsubsection{Proton Source}

The Proton Source delivers $8 \mathrm{GeV}$ protons to the Main Injector. It consists of the linear Pre-accelerator and Linac, and the circular booster.

The first accelerator is the Pre-accelerator. Negative hydrogen ions $\left(\mathrm{H}^{-}\right)$are accelerated to a kinetic energy of $750 \mathrm{keV}$ by an Cockroft-Walton accelerator. The Pre-accelerator produces bunches of $\mathrm{H}^{-}$ions at a rate of $15 \mathrm{~Hz}$.

Next, the Linear Accelerator or Linac accelerates the ions to $400 \mathrm{MeV}$ by Radio Frequency (RF) cavities, while the beam is focused by quadrupole magnets. Because the RF cavities have a fixed frequency and the particles accelerate while traversing the Linac, the cavities have increasing drift tube length.

The next step is the Booster, which is the first synchrotron in the acceleration chain. The ions are injected in the Booster and pass through a so-called stripping foil, which collects the electrons. The protons continue and are bent by magnets to a circular orbit of $75 \mathrm{~m}$ radius. Because the protons have positive charge, they can be kept in the Booster with the same magnet with which the $\mathrm{H}^{-} \mathrm{s}$ are injected. To ensure acceleration and a stable orbit, the magnets and RF cavities need to 


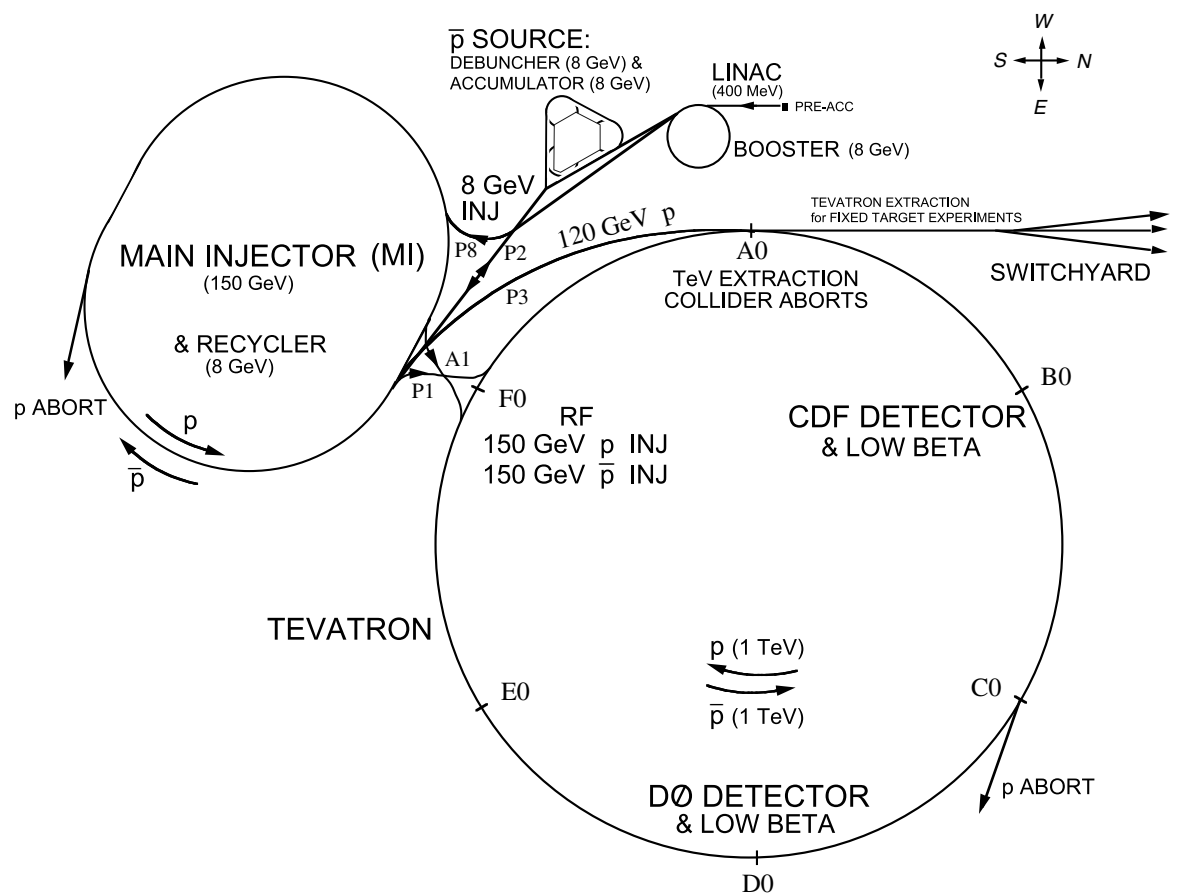

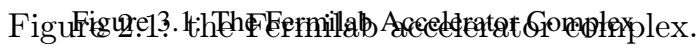

\subsubsection{Proton production and initial acceleration}

be synchronized with the proton bunches, giving it the name synchrotron.

Production of colliding beams starts with negative hiydrogen ions $\left(\mathrm{H}^{-}\right)$produced in a magnetron surface plasma source. The ions are produced from the interactions of electrons in the plasma with

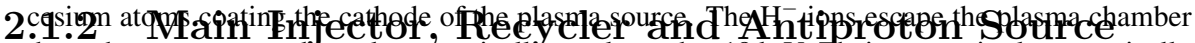
through an aperture and are electrostatically accelerated to $18 \mathrm{keV}$. Their energy is electrostatically

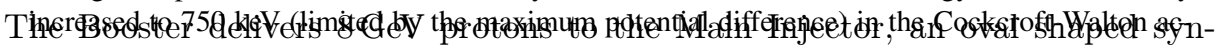

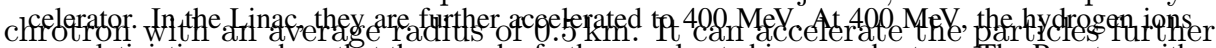
are. relativistig enough so that they can be further accelerated in a synchrotron. The Booster, with to either er or $495 \mathrm{~m}$, strips the hydrogen lons of their electrons by passing them through a-

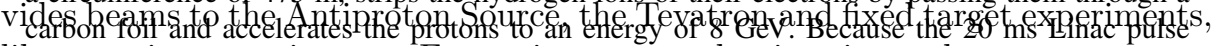

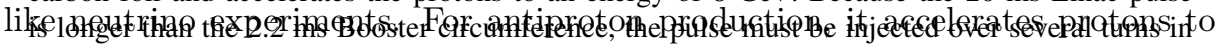

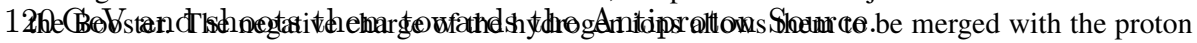

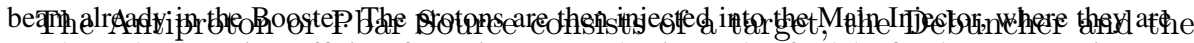

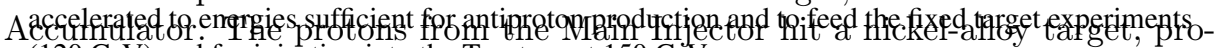

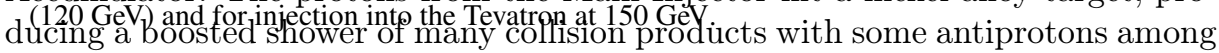
them. It takes about $10^{5}$ protons with $120 \mathrm{GeV}$ to make an antiproton with an energy around $8 \mathrm{GeV}$. Magnets behind the target are used to select particles with 
the right momentum and negative charge. These are fed into the Debuncher, a synchrotron with the shape of a rounded triangle and a mean circumference of $560 \mathrm{~m}$. Its main goal is to cool the antiproton beam by bunch rotation and stochastic cooling.

The beam is then transferred to the Accumulator, another synchrotron in the same tunnel as the Debuncher. It acts as a storage ring for the antiprotons, where they are further cooled. During the time they are stored, other particles with the same momentum-over-charge ratio as the antiprotons but a finite lifetime have the chance to decay, purifying the beam.

Housed in the same tunnel as the Main Injector, the Recycler stores antiprotons from the Accumulator and cools them further. Its other original purpose was to recycle the antiprotons from the Tevatron that remain after a store, but this was abandoned after problems in the beginning of Run II. Besides stochastic cooling, the Accumulator uses electron cooling, where a concentrated beam of cold electrons with the same average velocity is put in the beam along with the antiprotons. The antiprotons transfer some of their heat to the cooler electrons through soft scattering. Electron cooling is important when there are more than about $2 \cdot 10^{12}$ antiprotons in the Recycler, because stochastic cooling then becomes much less effective.

Once the $\bar{p}$ stash is big enough, it is split into 36 bunches and sent to the Main Injector. These bunches are accelerated to $150 \mathrm{GeV}$ and transferred to the Tevatron. Another 36 bunches, containing protons from the Booster, are also accelerated and delivered to the Tevatron. The (anti)proton bunches are split into 3 groups of 12 bunches. These groups, or trains, are $2.6 \mu$ s apart.

\subsubsection{The Tevatron}

As a synchrotron with a $1 \mathrm{~km}$ radius, the Tevatron is the largest accelerator of Fermilab. It accelerates the (anti)protons to $980 \mathrm{GeV}$ using its $8 \mathrm{RF}$ cavities, but afterwards the Tevatron is basically used as a storage ring. The beam is bent to a near circle by niobium-titanium magnets, which are cryogenically cooled to about $4 \mathrm{~K}$ to make them superconducting. The Tevatron is divided into six sectors with at the beginning of every sector one straight part, named A0 up to F0. The beams from the Main Injector enter the Tevatron at F0, which is also where the RF cavities are situated. The two interaction points are at B0 and D0, where the beam is optimally focused with low-beta quadrupoles, to get the highest luminosity. CDF is placed at B0, while the $\mathrm{D} \varnothing$ experiment was named after its location at the Tevatron, D0.

During a store the luminosity decreases nearly exponentially, due to the loss of particles from high-energy collisions and beam-beam effects. Once the luminosity of the store has decreased and a new stack of antiprotons is collected, the beams 


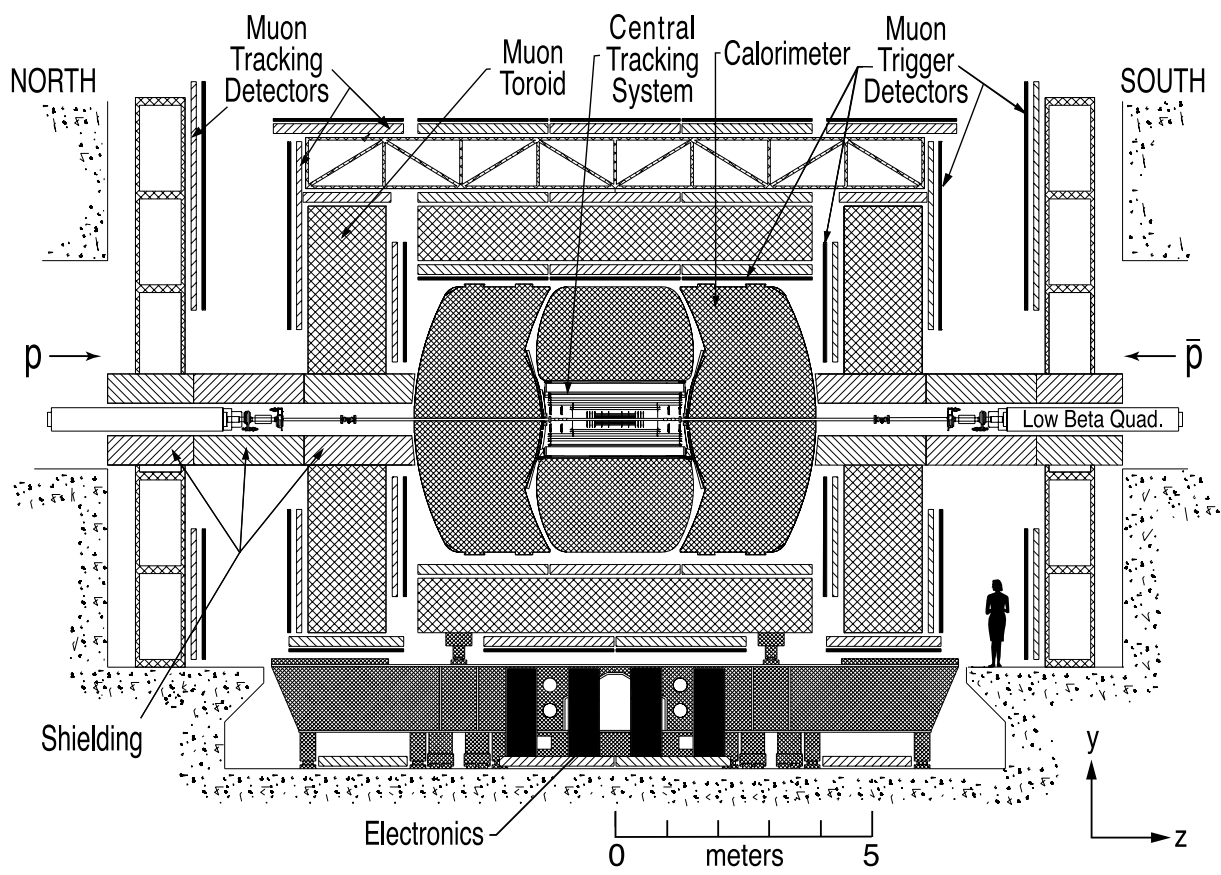

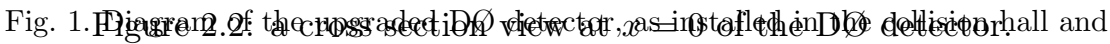
viewed from inside the Tevatron ring. The forward proton detector is not shown. The detectors in the central region of the detector are shown in Figure 2.

will be dumped and preparations for a new store begin. An average store lasts removal of the ol Mam Ring beam pipe from the caporimeters. Durmng Run I, the Main Ring was used to accelerate protons for antiprofon production while the Tevatron operated in collider mode. Losses from the Main Ring produced spurious energy deposits in the calorimeters and muon system, and most trig2.2 2 gers mere not mately $10 \%$, depending on the trigger.

To study the high-energy proton-antiproton collisions provided by the Tevatron,

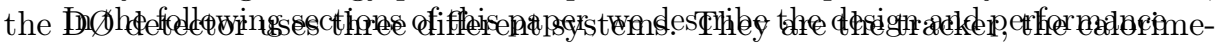

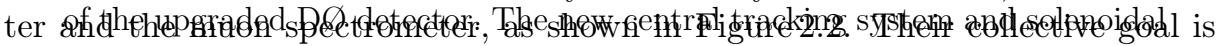

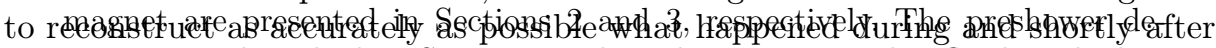
the toptorlisironsescribed in Section 4 . The calorimeters are briefly described in

Section 5 along with the new calorimeter electronics. The muon system is The enormous amount of data coming from the subdetectors is filtered and

processed by a three-level triggering system, while the Luminosity. Monitors measure the total interaction rate for the normalization of the cross sections

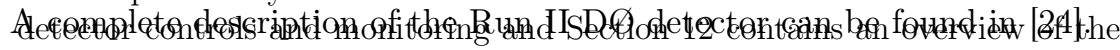
software components of the experiment. A list of acronyms appears at the end of the paper. 


\subsubsection{Transverse Momentum and Coordinate Systems}

One of the difficulties of hadron collisions is that because hadrons are composite particles, it is a priori unknown what constituents took part in the collision and what fraction of the hadron's energy and momentum they carried. This gives rise to large uncertainties in the reconstruction of the event. The center-of-mass frame of the collision is boosted by an unknown amount, making the assumption of a total momentum balance intractable. Because the boost can only occur along the beam direction, the transverse directions should have such a balance, so the transverse part of the momentum, $\vec{p}_{\mathrm{T}}$, is used. Usually, only the absolute value $p_{\mathrm{T}} \equiv\left|\vec{p}_{\mathrm{T}}\right|$ is mentioned, which should be zero for all the collision remnants combined. If there is a $p_{\mathrm{T}}$ imbalance, it is noted as missing transverse energy, $\mathbb{E}_{\mathrm{T}}$, which is the same as the combined $\vec{p}_{\mathrm{T}}$ of the entire detector, but with opposite direction.

DØ uses different coordinate systems: Cartesian, cylindrical and spherical coordinates. The direction of the proton beam (south) is defined as the $z$ axis, while the $x$ axis points away from the center of the Tevatron (east) and the $y$ direction is upward, making a right-handed Cartesian coordinate system.

In cylindrical coordinates, $x$ and $y$ are replaced by the radial direction $r \equiv$ $\sqrt{x^{2}+y^{2}}$ and the azimuthal angle $\phi \equiv \tan ^{-1}(y / x)$. Sometimes, the polar angle $\theta$, with $\cos (\theta) \equiv z / \sqrt{r^{2}+z^{2}}$, is also used.

Because of the unknown $z$ boost, it is often convenient to use the rapidity. Differences in this coordinate are invariant under Lorentz transformations along the beam pipe. For a collision product with energy $E$ and longitudinal momentum $p_{z}$, the rapidity is defined by

$$
y \equiv \frac{1}{2} \ln \left(\frac{E+p_{z}}{E-p_{z}}\right) .
$$

The particle is usually highly relativistic, so its mass is negligible and the rapidity can be approximated well by the pseudorapidity

$$
\eta \equiv \frac{1}{2} \ln \left(\frac{|\vec{p}|+p_{z}}{|\vec{p}|-p_{z}}\right)=-\ln \left(\tan \frac{\theta}{2}\right) .
$$

Even if the particle has a non-negligible mass, $\eta$ is a relevant substitute for $y$, because the mass is a priori an unknown.

The collisions typically take place in a region with a $25 \mathrm{~cm}$ length, because the (anti)proton bunches have a longitudinal spread. It is convenient to have the collision point as the origin, so we redefine the coordinates accordingly. Changes in for example $\eta$ are determined for each primary vertex. The coordinates mentioned in the rest of this chapter refer to the 'detector coordinates', which suppose a collision in the center of the detector. 


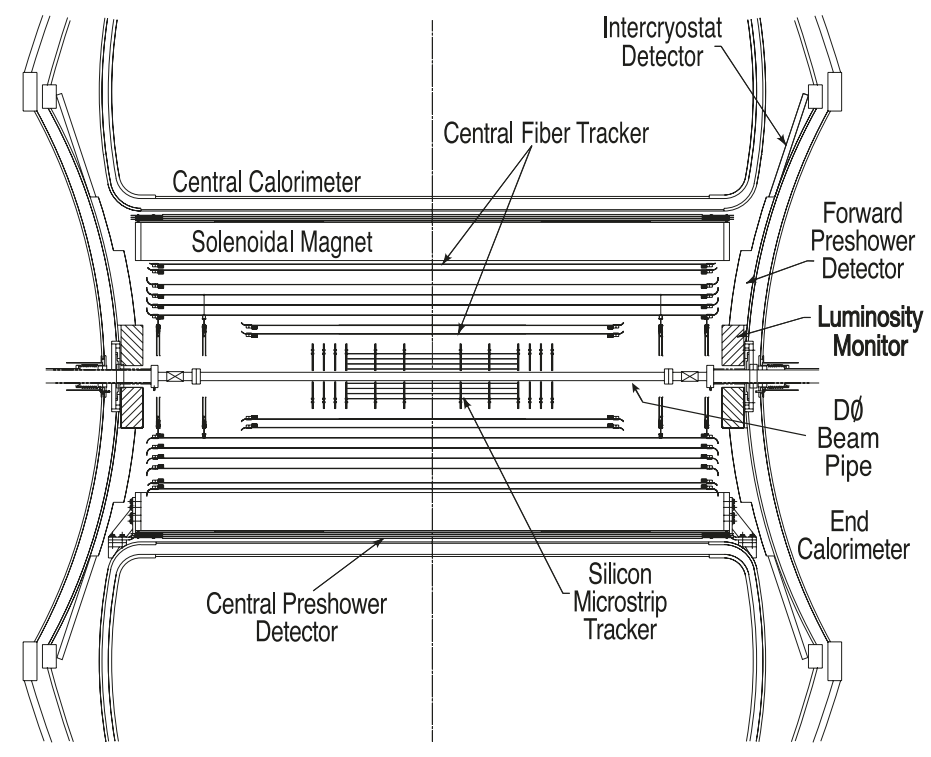

Figure $\begin{aligned} & \mathrm{Fig}_{2} \text { 2. Cross-sectional view of the new central tracking system in the } x-\text {. } z \text { plane } \\ & \text { Also }\end{aligned}$ monitor, and the calorimeters.

\subsubsection{Tracking}

The Tracker is an important part 106tathe detector, because it can determine the momentum of the charge 190 aticles tefaversins it. It can also identify secondary vertices, which indicates a⿳亠丷厂巾particle decay, as explained in Section 3.3

The tracking system Ensists of the Silicon Microstrip Tracker (SMT), the central fiber tracker (CFT), a solenoid magnet surrounding them and preshower detectors just outside the mpasnet. 'The solęnoid produces a $2 \mathrm{~T}$ magnetic field, curving the track of a charged particle to a helix with a radius proportional to

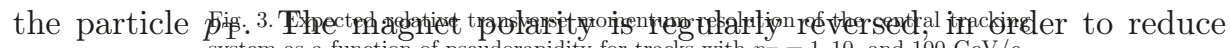
systematic uncertainties in the traction of peuding for

The layout of the central tracker is shown in Figure 2.3 .

\section{SMT}

12

To achieve the precise vertex resolution, high-accuracy tracking is needed close to the interaction point. Therefore the SMT, with a measured hit resolution of about $20 \mu \mathrm{m}$, was constructed just outside the beam pipe.

The SMT is basically a large number of closely-packed diodes, which give a signal when a charged particle passes through it, making electron-hole pairs. The diodes are combined to sensors by having silicon wafers with n- and/or p- 


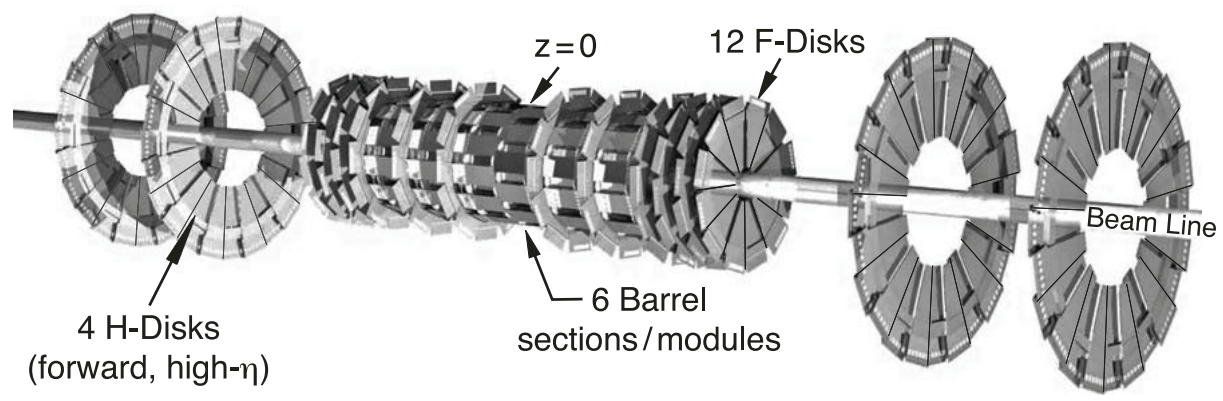

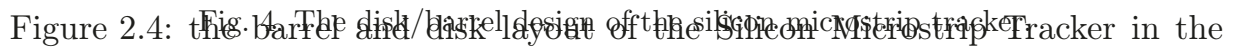
Run IIa configuration.

doped strips. Double-sided sensors have oppositely doped strips with a stereo

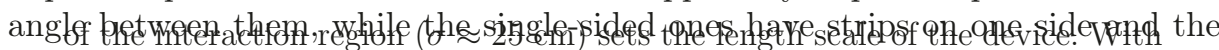

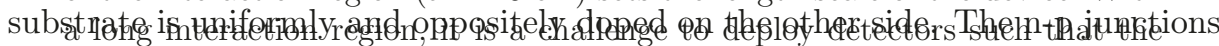

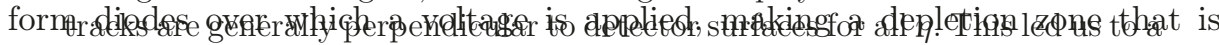

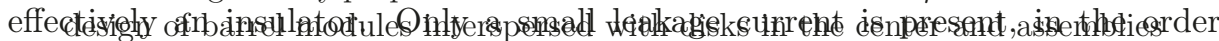

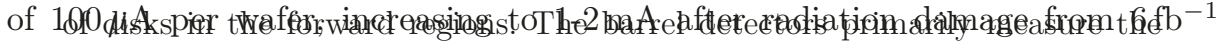

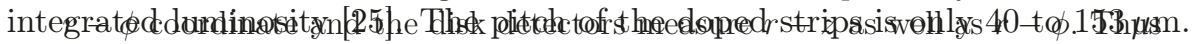

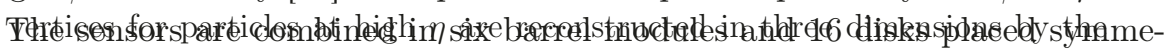

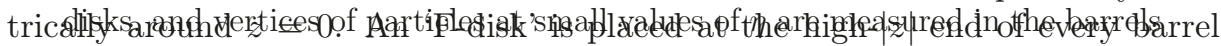

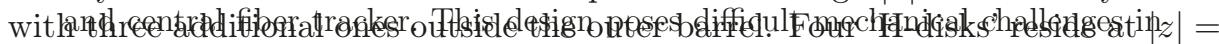
1.00аr ranting instanfficienter ppace disccissliagdanswcatphe configuration of the SMT is long in the $z$ direction, as can be seen in Figure 2.4. This ensures that high- $z$ vertices can still be reconstructed and that tracks with high $\eta$ also have hits close to their vertex. An isometric view of the SMT is shown in Figure 4. The detector has six

Each barre is $12 \mathrm{~cm}$ long and made with 4 lavers each an overlappin so set of two sublayers. Most lavers have two 6 cm sensors bonded together to form one 12 cm lad der, while davers 1 and 3 of the inner four barreds have $12 \mathrm{~cm}$ single-

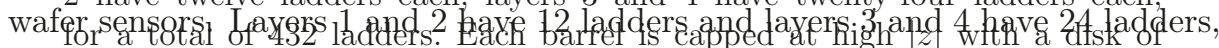

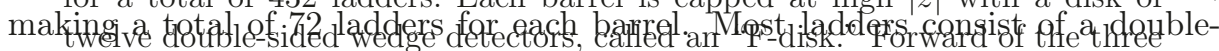

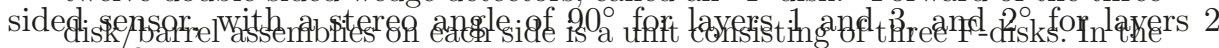

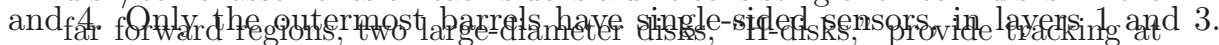

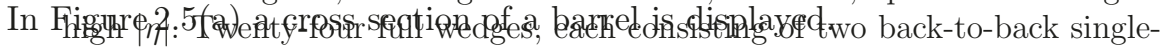

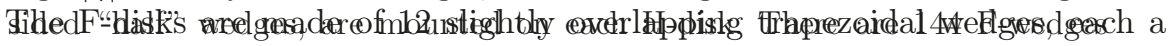

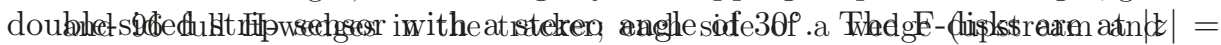

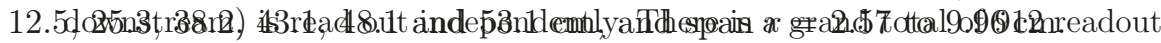

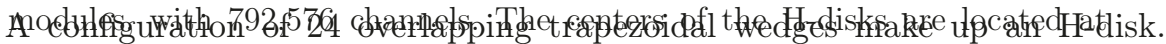

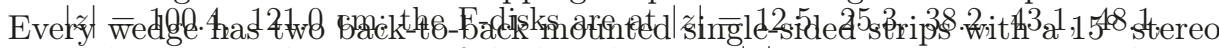
and $53.1 \mathrm{~cm}$. The centers of the barrels are at $|z|=6.2,19.0,31.8 \mathrm{~cm}$. The SMT is read out by custom-made 128-channel SVXIIe readout chips. 


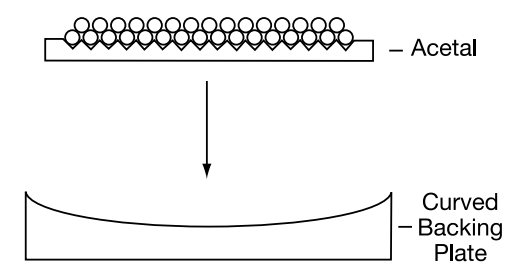

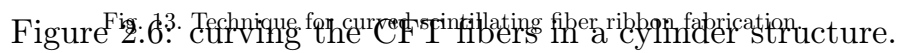

Table 2

Design parameters of the CFT; $u=+3^{\circ}, v=-3^{\circ}$. A through $\mathrm{H}$ correspond to the

CFT eight axial layers of the CFT.

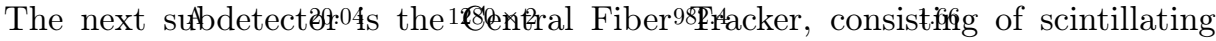
fibers. Whe raber2isenit by emitted in the form of fokight. Thine light travebsto the end of the fiber, where it is

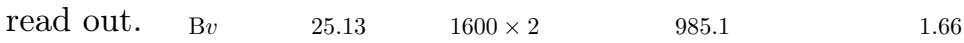

The CFE configuxation comsist $t_{2}$ of 8 congentric superlayers with $r \approx 20,25$,

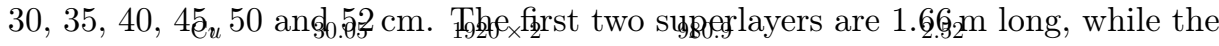
outer six are $2.52 \mathrm{~m}$ long.
$2240 \times 2$
974.4
2.52

Each superlayer çansists of towo tay $_{2}$ layers: angaxial $z$ layer, parallel to the beam axis, and a stereo layer with $\mathrm{a}_{2560}+3_{2}^{\circ}$ angle in the $\phi$ direction. ${ }_{2.52}$ Counting from the inside out, the odd superlayers, called $u$, have a positive stereo angle layer, while the even ones have a negative ${ }_{44.56}^{2560} \times 2$ angle layer, the $v$ layers. ${ }_{2.52}^{2.52}$ ery layer is made of $835 \mu \mathrm{m}$ diameter scintillating fibers, arranged in a zigzag pattern as shown in

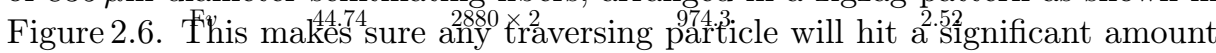
$\begin{array}{lllll}\text { of fiber material. } & 49.49 & 3200 \times 2 & 969.8 & 2.52\end{array}$

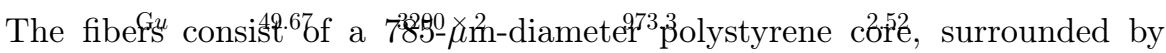

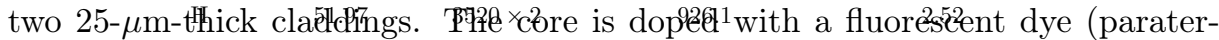

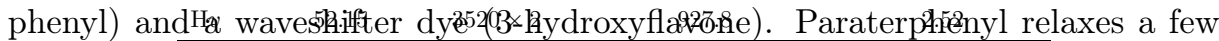
nanoseconds after a traversing particle excited it. The resulting $340 \mathrm{~nm}$ radiation would cylinder The grooved plastic vas inserted into a rigid, curved backing pate of

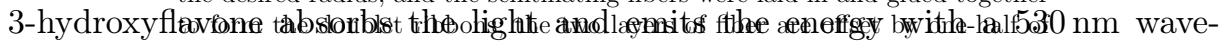

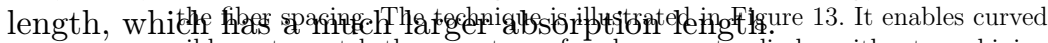

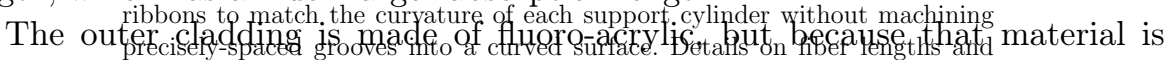
mechanicallbadiagompatibled withbe paraterphenyl, an inner cladding of polymethylmethacrylate is used. The core, inner cladding and outer cladding have a refractive index of 1.59, 1.49 and 1.42 , xespectively.

At one end of the fiber (south for the axial layers, north for the stereo ones), the light is transferred to waveguides, which have the same composition as the scintillating fibers, but without the dyes. The other end of the scintillating fibers is coated with a $90 \%$ reflective aluminum coating, so most of the light emitted in the wrong direction is reflected and can be measured some nanoseconds later. 


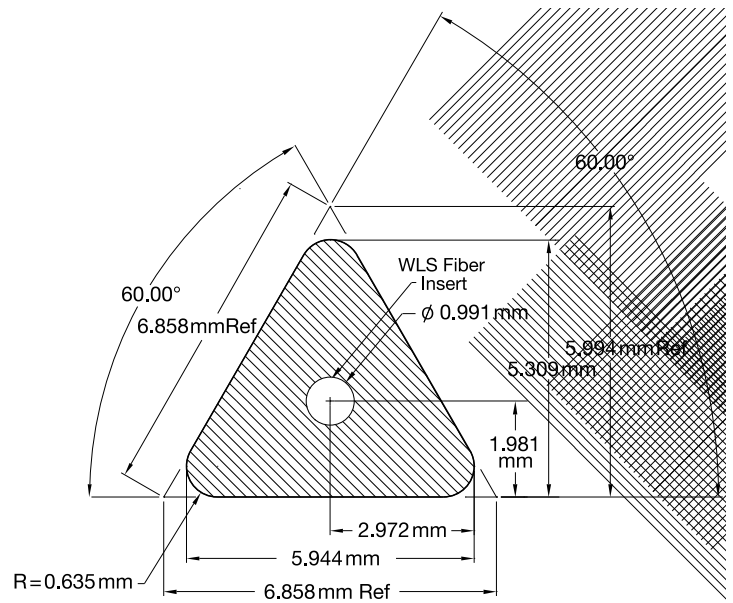

(a) scintillator fiber

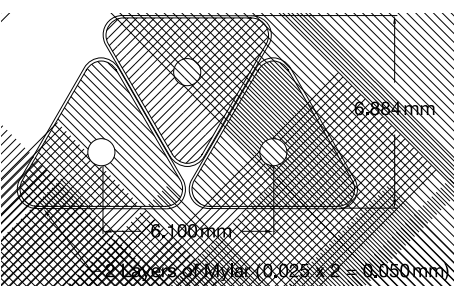

(b) CPS geometry

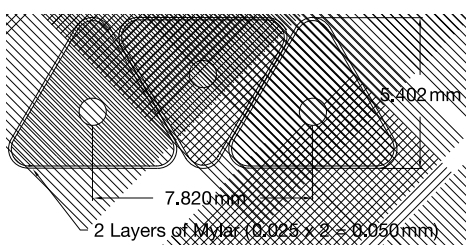

(c) FPS geometry

Figure 2.7: the preshower geometry of (a) a scintillator fiber, (b) the CPS layers and (c) the FPS layers.

A waveguide transports the signal to a visible-light photon counter (VLPC), which is a silicon pixel chip that operates at $9 \mathrm{~K}$. The VLPCs are connected to analog front-end (AFE) boards that determine the ADC (analog to digital converter) count. New AFEII-t boards were installed during an upgrade in 2007, which can record the time the signal first went over threshold. The time depends on the pulse height through two mechanisms, the photon-multiplicity effect and slewing. If more photons are produced, the chance that one or more photons starts in the direction of the readout is larger, making the recorded time lower. Also, if the signal is higher, it will cross the threshold sooner, reducing the recorded time again. The timing is corrected for both effects, as explained in Section 3.2 .

The total $\phi \times r \times z$ resolution per hit for the CFT is about $0.1 \times 0.1 \times 20 \mathrm{~mm}^{3}$.

\section{Preshower Detectors}

The function of the preshower detectors is partially tracking, partially calorimetry; they are used to measure the tracks of particles after they traversed the magnet, and provide corrections to the energy measurement of the calorimeter. The preshower also aids in particle identification, because a heavy charged particle will be minimally ionizing both before and after it hits absorber material, while photons will only produce a signal (typically three fibers wide) behind an 
absorber, and electrons produce a minimally ionizing signal in front of the absorber, and a shower signal behind it.

The preshower detectors are situated between the tracker and the calorimeter and have of two parts: the Central Preshower Detector (CPS) and the Forward Preshower Detector (FPS). Both subdetectors are made of double layers of triangle-shaped fibers with a wavelength-shifting fiber in their core. In Figure 2.7(a) the fiber dimensions can be seen.

The fibers have a tracking resolution of about $1 \mathrm{~mm}$ [27] and the single-particle efficiency is about $95 \%$ for high $p_{\mathrm{T}}$ electrons [28].

The CPS covers the range $|\eta|<1.3$ and lies at $r=72 \mathrm{~cm}$ in the $5 \mathrm{~cm}$ space between the solenoid and the calorimeter. On the inside of the CPS, a lead slab is mounted with a thickness such that the amount of material, including the solenoid, is about 2 radiation lengths $\left(2 X_{0}\right)$ for particles coming from the detector center. There are three double layers in the CPS: one axial, one $u$ and one $v$ layer. The layers have a fiber layout as can be seen in Figure 2.7(b) and each layer is divided in eight modules. The stereo layers have an angle of $24^{\circ}$ in the $\phi$ direction.

The FPS is made of 4 double-fiber planes, with layout shown in Figure 2.7(c). The two closest to the interaction point are called minimum ionizing particle (MIP) layers and the other two are the shower layers. Between the MIP and shower layers lies a $2 X_{0}$ absorber, made of lead and stainless steel. There is a $u$ and a $v$ plane in both the MIP and the shower part of the FPS. The planes are divided into 8 wedges of $22.5^{\circ}$, while the absorber is made of 48 segments.

\subsubsection{Calorimetry}

By measuring the energy of electrons, photons and jets, the calorimeter is used in particle identification. The calorimeter can also determine whether there are high-energy neutrinos in the event, because they don't interact with the detector, causing $\notin_{\mathrm{T}}$.

Calorimetry is performed using interlaced plates of absorber material and signal boards, with active material in between, as can be seen in Figure 2.9(a). Particles traversing the calorimeter produce secondary-particle showers in the absorber plates. These showers ionize the liquid argon, producing a number of electrons proportional to the energy of the particles in the shower. The electrons drift to a signal board, which integrates their charge.

Hadron showers include many neutrons, which will only be detected once they free ionizing particles, for example by elastic collisions with protons in hydrogen nuclei. This means that, given the short integration period available for Run II, 


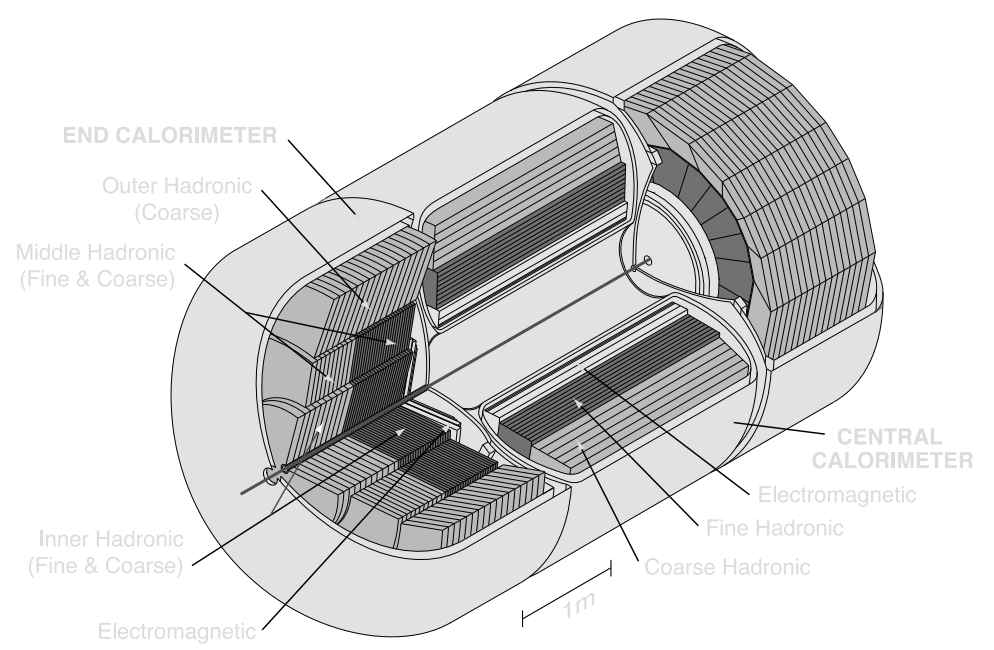

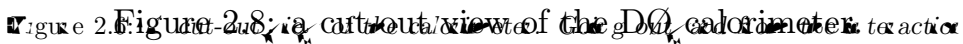

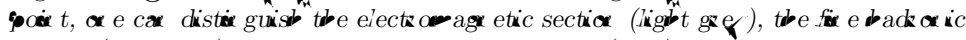
sectio (day, gre), a d the coase badk ic sectio (gx e).

the electron response of the neutrons is lower than that of EM particles. This

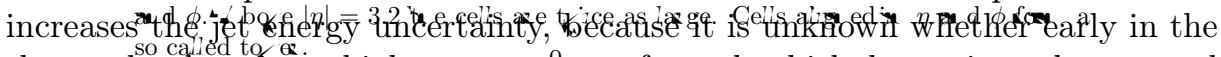
shower developmbnt

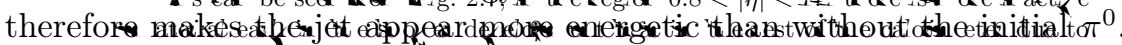

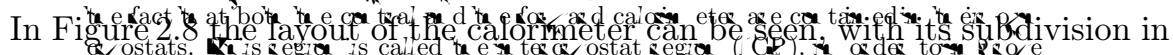

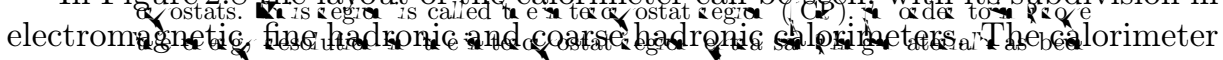

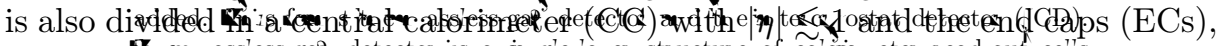

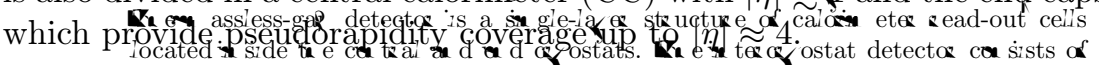

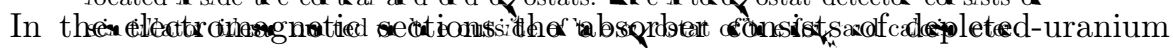

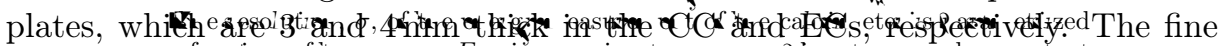

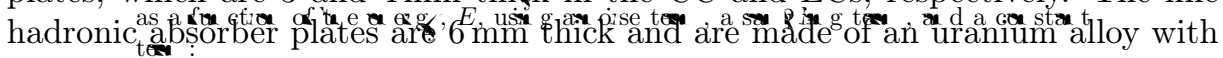
$2 \%$ niobium. The plates of the foarse caborimeters are much thicker: $46.5 \mathrm{~mm}$. They are made from copper in the

Liquid argon is used as active matergial, which means the calorimeters have to be cooled. The CC and the two ECs each have their own cryostat to keep the temperature at around $90 \mathrm{~K}$.

Two $0.5 \mathrm{~mm}$ sheets of G-10 plastic around a copper pad make up one signal board, which is coated with high-resistivity surface to serve as electrodes. A $2 \mathrm{kV}$ ( voltage is applied between these surfaces and the absorber plates.

Several unit cells are combined into a readout cell, while the readout cells of similar $\eta$ and $\phi$ are grouped into readout towers, as can be seen in Figure 2.9(b). The $\eta \times \phi$ width of a tower is about $0.1 \times 0.1$, except for the third layer of the EM 


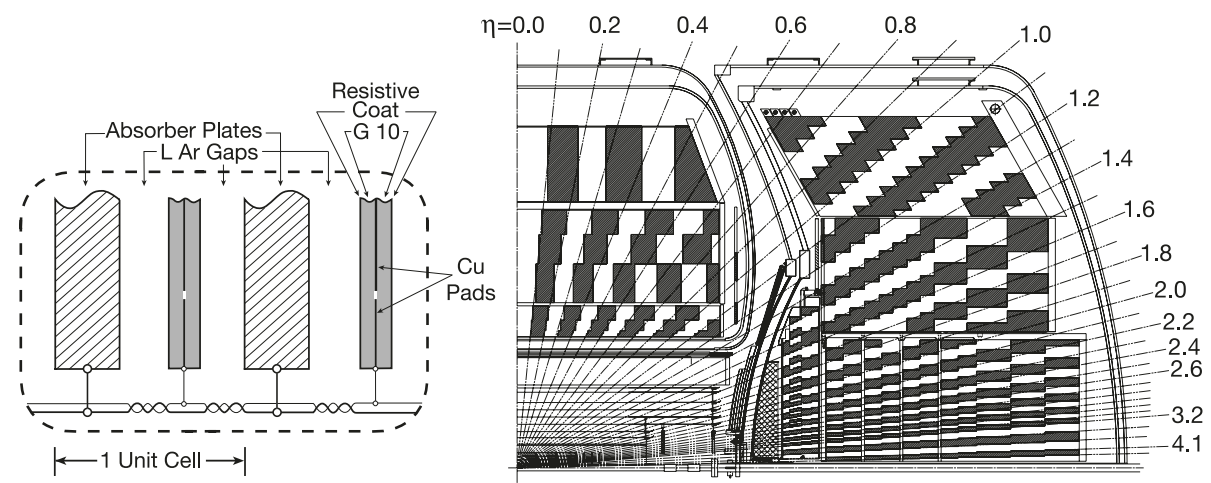

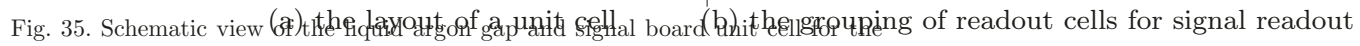
calorimeter.

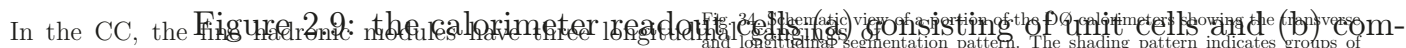

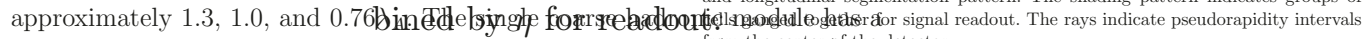

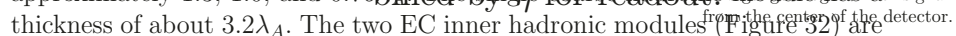

cylindrical, with inner and outer radii of 3.92 and $86.4 \mathrm{~cm}$. Threcfilherekadkorqimected by plated-through holes. The signals from these portion consists of four readout cells, each $1.1 \lambda_{A}$ thick. The multilaver boards in the EM and small-angle hadronic modules are ganged

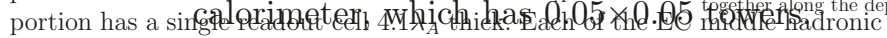

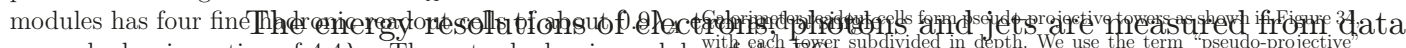

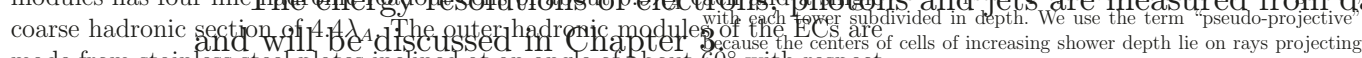

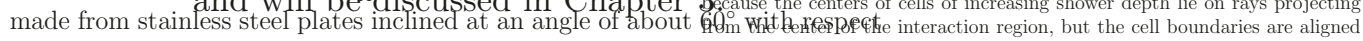

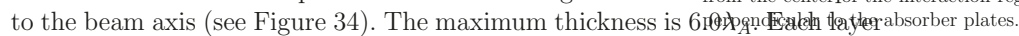

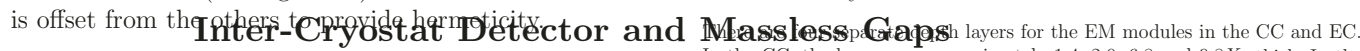
In the $\mathrm{CC}$, the layers are approximately $1.4,2.0,6.8$ and $9.8 X_{0}$ thick. In the

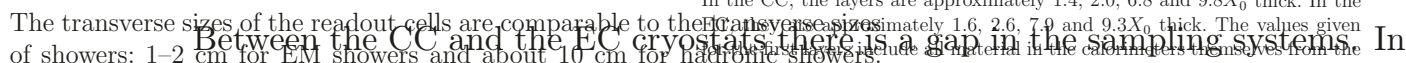
Towers in both this regiam,

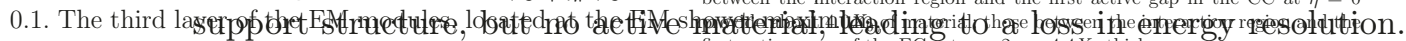

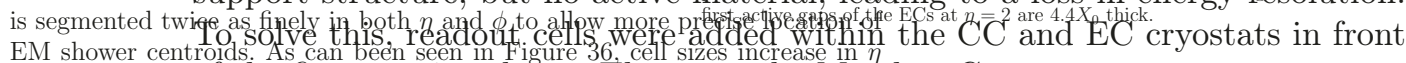

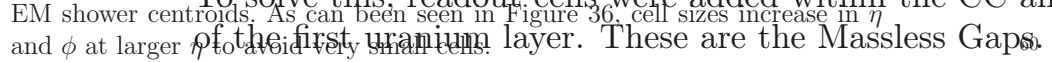

The Inter-Cryostat Detector (ICD), too, was built to provide active material.

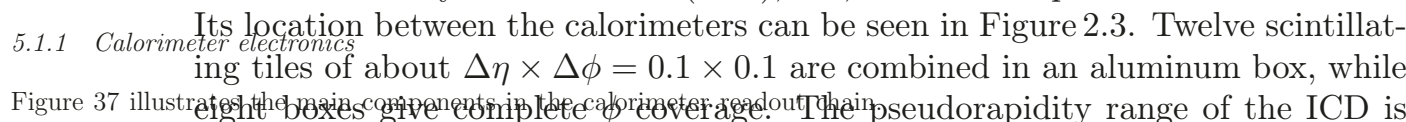

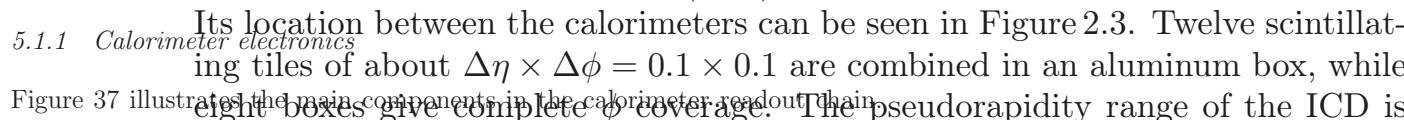
There are 55,296 calorimeter electonics channels to bead out; 47032 correspond to channels connected to physical readout modules in the cryostats. The h ibers, which transport the light

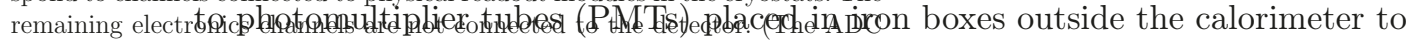

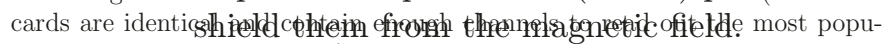

lated regions of the detector.) The readout is accomplished in three principal stages. In the first stage, signals from the detector are transported to charge

\subsubsection{The Muon System}

Muons are the only particles that traverse the calorimeter without losing most of their energy. This makes fast triggering on them possible, which is useful because high-momentum muons are an indication of interesting events. 


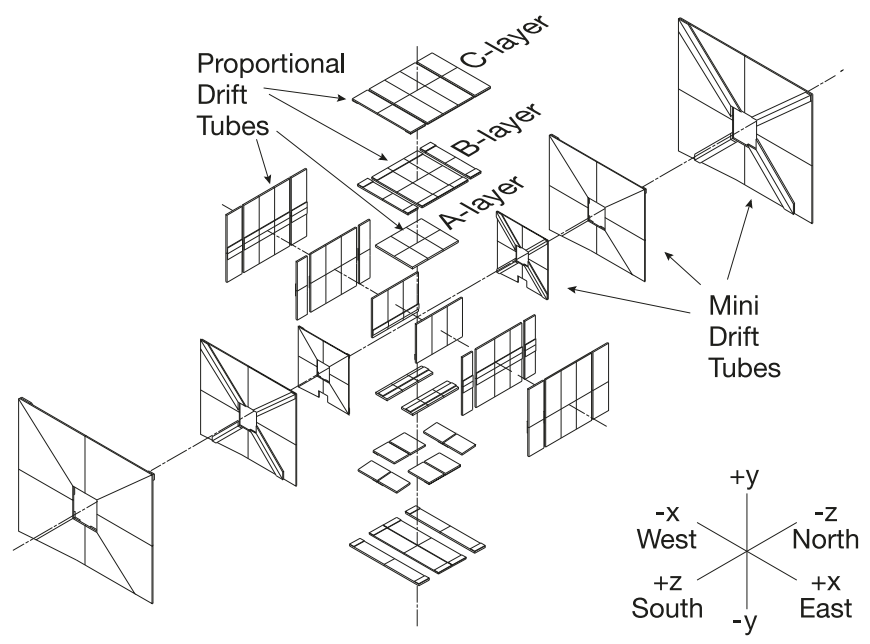

Figure 2.10: an exploded view of the the muon dire chambers thift tube detectors.

allow us to associate a muon in a PDT with the appropriate bunch crossing and to reduce the cosmic ray background. Additional scintillation counters, the $A \phi$ counters, have been installed on the PDTs mounted between the calorimeter

Muon spectrom the toroidal magnet. The Accounters provide a fast detector. for triggering scintillation counters. Like the solenoid, the toroid polarity periodically changes.

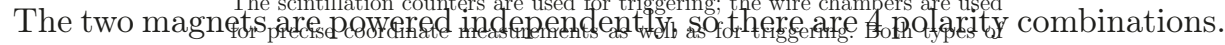

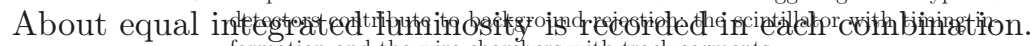

The central muon system has $|\eta| \stackrel{<}{<} 1$ and uses Proportional Drift Tubes (PDTs), while tew defectors and themodifications made to the roriginal systom are dischyssgd has Mini Drift

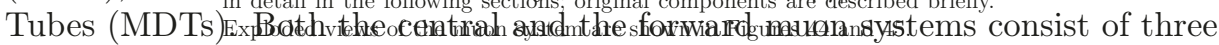
multilayers of drift tubes and scintillation counters. The drift tube multilayers are called A, P.1androdal firgmetinside out, as can be seen in Figure2.10, with the toroidal magnet placed between layers A and B. Layers B, C and the bottom

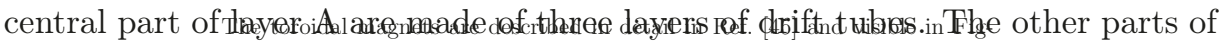
layer A have fours layers. wires with a gas mixture, which is different for the PDTs and the MDTs.

$$
74
$$

\section{PDT}

The PDT gas mixture, made of argon (84\%), methane $(8 \%)$ and tetrafluoromethane $(8 \%)$, flows through the tubes with a total refresh rate of every 8 hours. The cross section of the PDTs is 10 by $5.5 \mathrm{~cm}$, and with a drift velocity of about $10 \mathrm{~cm} / \mu$ s the drift resolution is about $0.5 \mathrm{~mm}$, while the resolution along the tubes is 10 to $50 \mathrm{~cm}$. The PDT layers are combined in large drift chambers with dimensions $2.8 \times 5.6 \mathrm{~mm}^{2}$. 


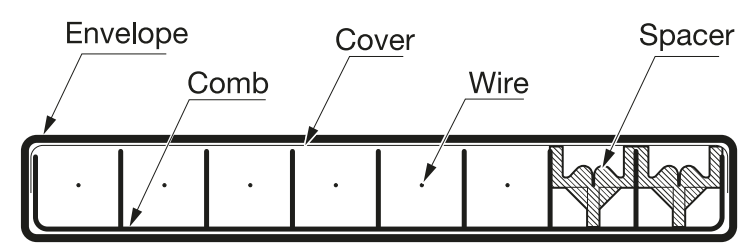

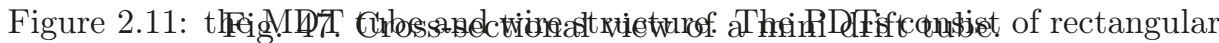
tubes with a suspended wire in their center.

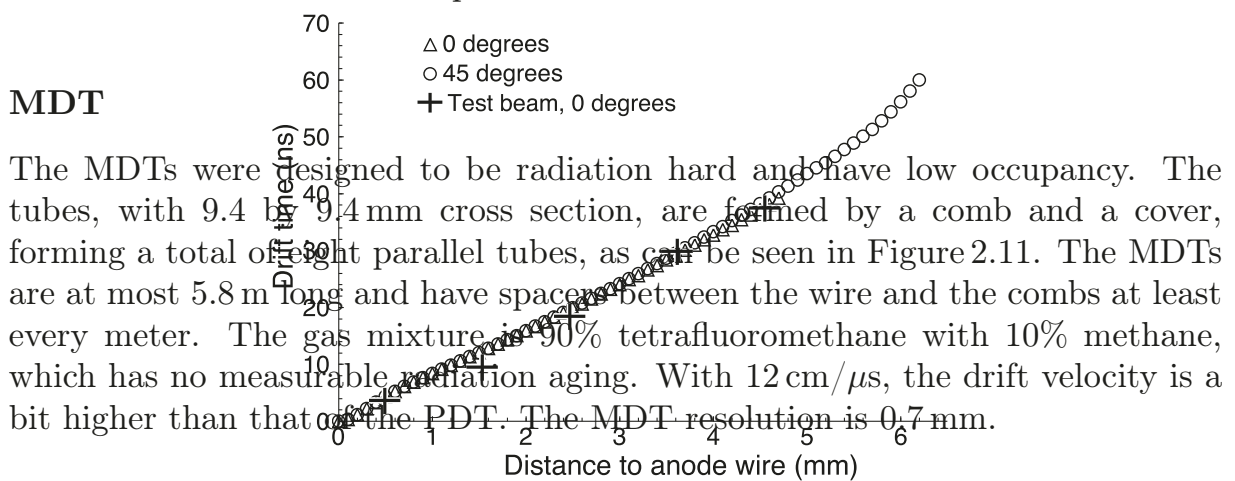

\section{Scintillation Counters}

Fig. 48. Time-to-distance relationship for a mini drift tube. The points labeled

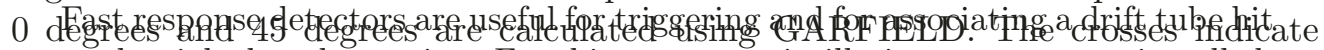
measurementight dunch atrpssing. For this purpose scintillation counters are installed.

In the central region they consist of 'cosmic caps' and 'A $\phi$ counters', while the

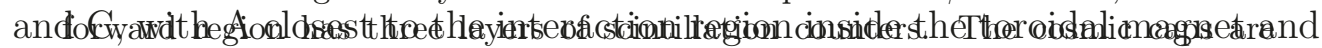

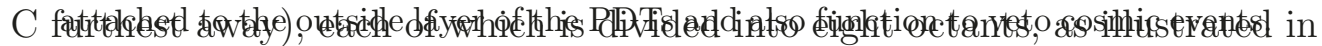

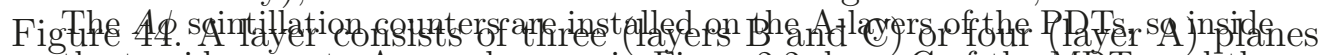
of the toroid magnet As can be seen in Figure 2.2 laye fof of the MD Ts and the outer layer of the forward scintillation counters is not mounted on the outside of.

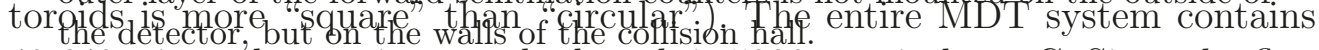
48,640 wires; the maximum tube length is $5830 \mathrm{~mm}$ in layer C. Since the flux of particles frops. with increasing distance from the beam line, the occupancy of individual tubes is the same within a factor of two over an entire layer.

It is very important to know the total luminosity at the DØ interaction point,

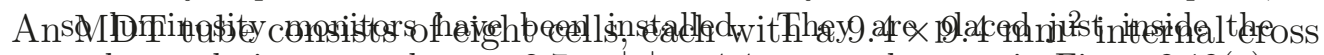

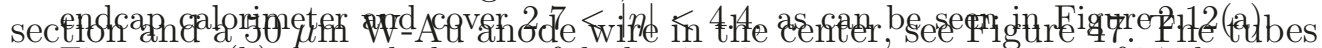
Figure 2.12(b) shows the layput of the luminpsity monitors, consisting of 24 plastic are niantillator wedges, connected to puls. Because of the r mision commesic field at the

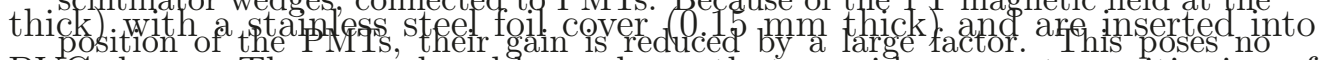

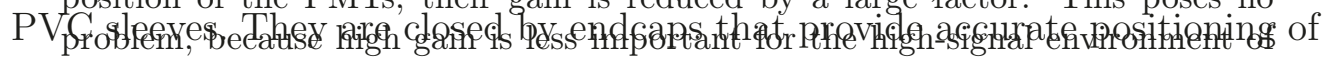

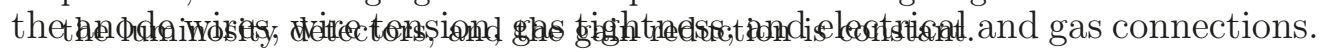
The anode wires are supported by spacers; the unsupported wire length never exceeds $1 \mathrm{~m}$.

The MDT system uses a $\mathrm{CF}_{4}-\mathrm{CH}_{4}$ (90\%-10\%) gas mixture. It is non-flammable, fast, exhibits no radiation aging, and has a wide operational plateau. The maximum drift time for tracks that are perpendicular to the detector plane is $40 \mathrm{~ns}$; for tracks inclined at $45^{\circ}$, the maximum drift time is 60 ns. Figure 48 shows the time-to-distance relationship for inclinations of $0^{\circ}$ and $45^{\circ}$ calculated using GARFIELD [94] and for test beam data. 


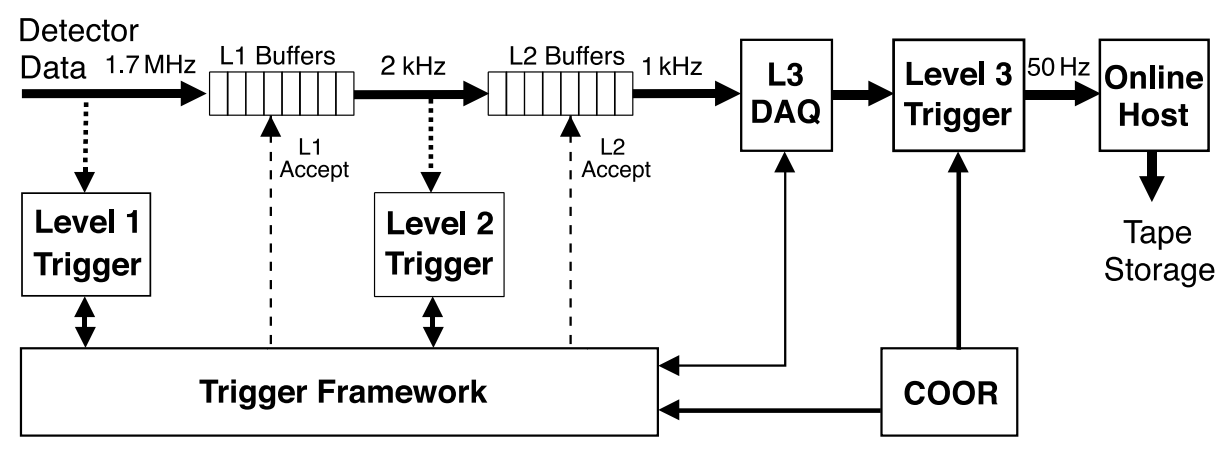

Figure 2.13: the DØ trigger and data acquisition systems. Fig. 59. Overview of the $\mathrm{D} \varnothing$ trigger and data acquisition systems.

\section{Triggering}

The third level trigger is software based and combines the information of all subdetector systems. Basically, it is a fast version of the offline reconstruction

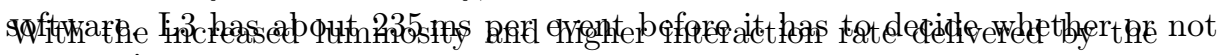
topgtareedt.Tevatron, a significantly enhanced trigger is necessary to select the

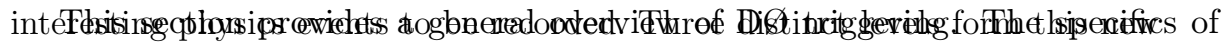

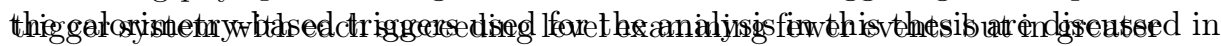
dectaidnand. Whith more complexity. The first stage (Level 1 or L1) comprises a collection of hardware trigger elements that provide a trigger accept rate of about $2 \mathrm{kHz}$. In the second stage (Level 2 or L2), hardware engines and embed-

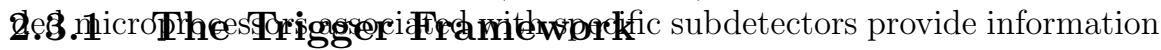
to a global processor to construct a trigger decision based on individual ob-

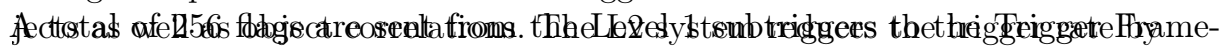

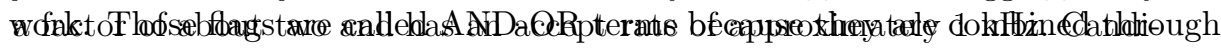

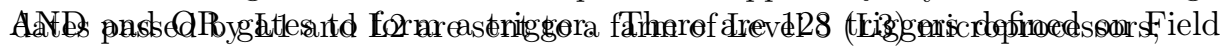

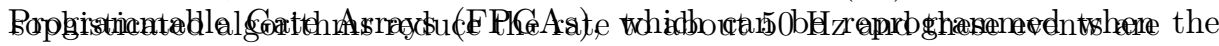

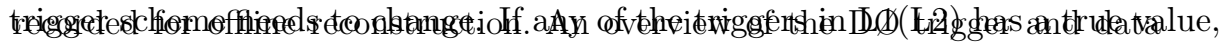

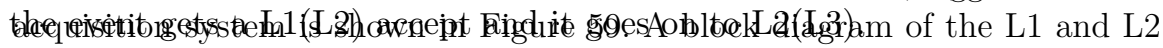

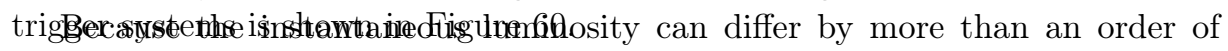
magnitude, the TFW uses a prescaling factor for some trigger terms. Prescaling

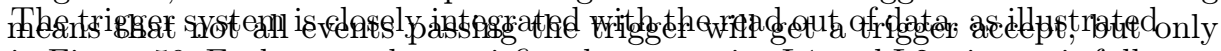

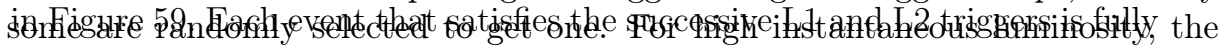

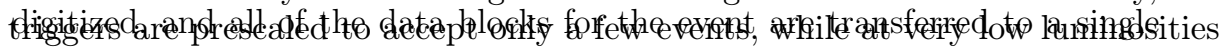

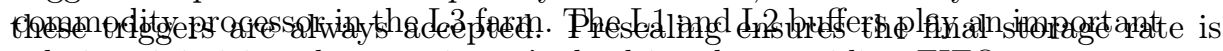

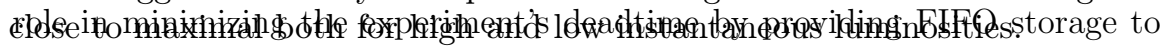

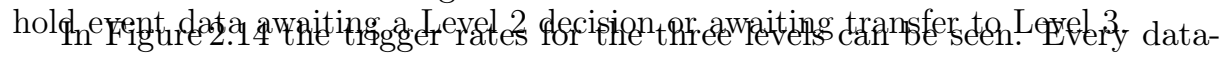
taking run the prescales in the TFW are altered, to keep the L3 accept rate close The $200^{\mathrm{V} e f a l l}$ coordination and control of D $\varnothing$ triggering is handled by the COOR package (Section 10.2) running on the online host. COOR interacts directly with the trigger framework (for L1 and L2 triggers) and with the DAQ supervising systems (for the L3 triggers). The data acquisition system responsible for the data flow of the fully digitized event into L3 is described in Section 10. 


\subsubsection{Level 1}

Triggering at the first level is done separately for the muon system (L1MUO), the calorimeter (L1CAL) and the tracking system (L1CTT). The triggers are a combination of hardware and FPGAs.

L1CAL has four readout towers combined to make a trigger tower, with $\eta \times \phi \approx$ $0.2 \times 0.2$. How many of the 1280 trigger towers went over threshold can be a trigger requirement. Also, the transverse energy of a group of $4 \times 8$ trigger towers are combined to preselect in the search for possible jets, which usually cover a couple of trigger towers. There are also two global L1CAL variables passed to the TFW: the sum over all tower transverse energies, $\sum E_{T}$, and the $\mathbb{E}_{\mathrm{T}}$.

L1CTT combines input from the CFT and the preshower detectors. Predefined track equations are used to find tracks in each of the 4 bins in transverse momentum: $1.5-3,3-5,5-10$ and $>10 \mathrm{GeV}$. Up to 480 tracks are sent to the L1MUO.

L1MUO uses the muon system with input from L1CTT tracks. The three pseudorapidity regions of the muon system (one central and two forward) are divided into eight $\phi$ sectors. If there is a coincidence in two layers with the same $\phi$ and $\eta$ sector, a check is performed whether the timing is consistent with a beam crossing to reduce cosmics. A matching track will be sought in the tracks coming from the L1CTT.

\subsubsection{Level 2}

The subsystems of Level 2 consist of the muon system (L2MUO) and the calorimeter (L2CAL), but here the tracking is differentiated between the SMT information (L2STT) and the combined tracking (L2CTT). The preshower trigger (L2PS) identifies electrons and photons, while rejecting charged hadrons. It does so by looking at the early shower development, as discussed in Section 2.2.2.

In the calorimeter part of the Level 2 trigger, simple clustering algorithms find jets and electrons or photons. The jet algorithm first finds trigger towers with $E_{\mathrm{T}} \geq 2 \mathrm{GeV}$, which are used as seeds. Trigger tower clusters of $5 \times 5$ are made around the seed towers. If two jets have more than 4 overlapping towers they are merged. The EM algorithm takes the EM towers with $E_{\mathrm{T}}>1 \mathrm{GeV}$ as seeds and uses $3 \times 3$ clusters around those towers.

L2STT uses L1CTT tracks and reconstructs them with much higher precision. At this point the impact parameter can already be determined accurately enough to identify possible long lived particles, like $b$-quarks. The findings of the L2STT 
are taken as input for the L2CTT, which compares the azimuthal angle $\phi$ in the beginning of the track with its value on the outside of the CFT. This is a measure of how much the track was bent by the solenoidal magnetic field and therefore of the track $p_{\mathrm{T}}$.

The improvement of L2MUO over L1MUO is the inclusion of calibration and improved timing information, improving the track quality of muon candidates.

In order to test for correlations between subdetectors the different L2 subsystems output to L2Global, which presents its findings to the TFW.

\subsubsection{Level 3}

L3 is a set of software triggers, that run on a computing farm with about 300 commercial PCs. Complete objects are reconstructed and relations between the physics objects can be used, like the combined invariant mass or angular differences.

Track segments are found by requiring two hits in the outer CFT layers that are compatible with high- $p_{\mathrm{T}}$ tracks. Then, the segments are propagated inward, into the SMT.

Muons are track segments in the muon system, with timing information included to check their consistency with the $p_{\mathrm{T}}$ of the muon tracks. Using vertex constraints and tracks from the central tracker, a higher momentum resolution can be attained than in L2. If a muon is isolated (away from a jet), a MIP signal is expected in the calorimeter, so this is also used.

Jets are reconstructed with a precise $p_{\mathrm{T}}$ measurement using the highestgranularity calorimeter output, corrected by hot-cell suppression. Also the $z$ position of the vertex is used, for it alters $\theta$ and therefore the $E_{\mathrm{T}}$. The total scalar $E_{\mathrm{T}}, \mathbb{E}_{\mathrm{T}}$ and the $\mathbb{E}_{\mathrm{T}}$ significance are subsequently recalculated.

Electrons are defined by a $\Delta \eta^{2}+\Delta \phi^{2}=0.0625$ cone with an EM fraction in the calorimeter higher than 0.9 . The L3 trigger can also demand a corresponding preshower signature.

\subsection{Monte Carlo Simulations}

In order to compare theory with experiment, the high-energy collisions are simulated by Monte Carlo (MC) methods, as described in Section 2.4.1. The description how the detector response is simulated can be found in Section 2.4.2 and how the collision-independent background, the 'underlying event', is modeled in Section 2.4.3. In Section 2.4.4 reweightings are discussed for situations where the $\mathrm{MC}$ does not describe the data. 


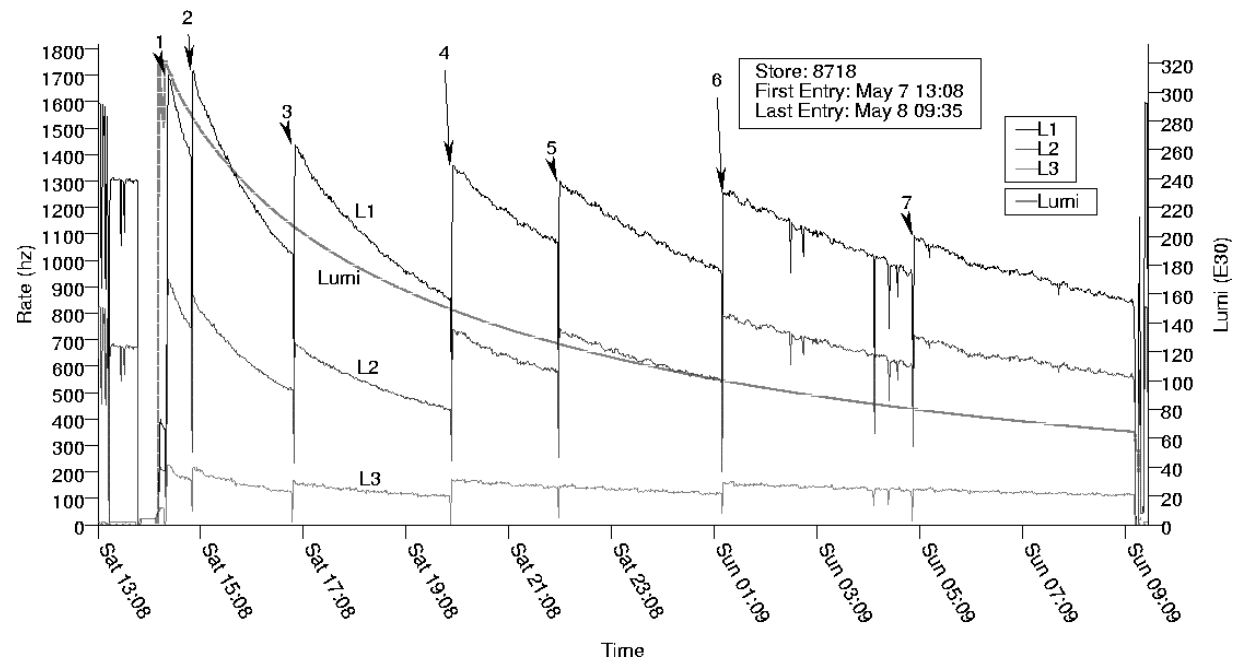

Figure 2.14: the luminosity and L1, L2 and L3 trigger rates of a typical store. The numbers 1 to 7 indicate the start of different data taking runs.

\subsubsection{Event Generation}

MC methods generate events with a distribution in particle content and phase space that matches the theory prediction. There are several elements of event generation:

the hard process, which covers the high-energy interaction of interest, for example the creation of massive gauge bosons, top quarks or Higgs bosons;

parton showering, describing how partons (gluons or quarks) split into more partons due to the strong interaction. This happens many times, because of the large coupling constant; and

hadronization, which forms jets of color-neutral hadrons from the color-charged partons. Because of the nature of the strong interaction, it is energetically more favorable for new quark-antiquark pairs to be created from the vacuum than for color charges to be separated by a large distance. Therefore, a stream of hadronic, color-neutral particles, a jet, is formed for every highenergetic, color-charged particle.

\section{In the $W H \rightarrow \tau \nu b \bar{b}$ Analysis}

For the analysis presented in this thesis, each high-energy process has been modeled using the MC algorithm that performs optimally for that process. The 


\begin{tabular}{llll}
\hline Event type & $K$-factor & Event type & $K$-factor \\
\hline$W+$ l.f. & 1.3 & $W W$ & 1.0 \\
$W+b / c$-jets & $1.3 \times 1.47$ & $W Z$ & 1.06 \\
$Z+$ l.f. & 1.3 & $Z Z$ & 1.03 \\
$Z+c$-jets & $1.3 \times 1.67$ & $t \bar{t}$ & 1.43 \\
$Z+b$-jets & $1.3 \times 1.52$ & single top & 0.99 \\
\hline
\end{tabular}

Table 2.2: $K$-factors used for MC event generation. There is a overall $K$-factor for all $W / Z+$ jets events, 1.3 , and an additional factor when heavyflavor jets are produced.

signal events $(W H$ and $Z H)$ are simulated with PYTHIA [29]. Also di-boson events ( $W W, W Z$ or $Z Z$, possibly with accompanying jets) are generated with PYTHIA. All events with one massive vector boson and jets $(W / Z+$ jets $)$ as well as those with a pair of top quarks $(t \bar{t})$ are produced with ALPGEN [30, interfaced with PYTHIA for the simulation of the parton showering and hadronization. The $W / Z+$ jets events are split into light-flavored ones $(W / Z+$ l.f. $)$, with only light partons (gluons or $u, d$ or $s$ quarks), and ones with heavy-flavored partons ( $c$ or $b$ quarks), called $W / Z+b / c$-jets. The fraction of heavy versus light-flavored quarks is obtained using MCFM [31]. Events with a single top quark are generated by COMPHEP 32 .

Next-to-next-to-leading order (NNLO) predictions are used to determine the overall cross sections. This results in correction factors, so-called $K$-factors, that alter the normalization of the processes, but do not affect their shapes. In Table 2.2 the applied $K$-factors are listed. There is a residual uncertainty, which is treated as a systematic, as further explained in Section 5.2

\subsubsection{Detector Simulation}

All particles that are produced by the event generators are propagated through the detector and their interaction with the detector material is simulated. This is done using d0gstar [33, the $\mathrm{D} \varnothing$ implementation of the detailed detector-material simulation GEANT3 34. The electronics and trigger responses are then simulated with d0sim [35] and d0TrigSim [36].

\subsubsection{Underlying Event}

The underlying event describes collision independent backgrounds. This includes the interactions of the remnants with products from the hard collision, remnantremnant interactions, pileup from the previous bunch crossing and pileup from 
multiple collisions during one bunch crossing. The underlying event also models detector noise, such as neutrons coming from longer-term radioactivity of the calorimeter.

In $\mathrm{D} \varnothing$, the underlying event is described by zero- and minimum-bias data that have been overlaid on the MC production. Zero-bias triggering includes all events, while minimum-bias data are triggered by the luminosity monitor, indicating there was at least one $p \bar{p}$ collision. Both the zero- and the minimumbias triggers have a large prescaling factor to ensure feasible data rates.

\subsubsection{MC reweighting}

Variable distributions of MC simulations can have different shapes than those of measured data. In that case, a so-called reweighting can be applied, meaning that the weights of the MC events are multiplied by a factor depending on the value of the mis-modeled variable.

For example the average instantaneous luminosity of zero-bias and minimumbias events can be different from that of the regular data set, so the MC events have been reweighted in luminosity in order to match the data. This is also the case for the $z$ position of the primary interaction.

There are many more MC reweightings in different stages of the event reconstruction and the analysis. They will be discussed in Sections 3.6, 3.7, 4.1.2, 4.3 and 4.5.1. 


\section{Chapter 3}

\section{Event Reconstruction}

After an event is stored, it is passed through reconstruction software to identify tracks, vertices, muons, electrons, photons and jets. The identification of jets can be further refined by recognizing them as the decay of a $\tau$, or hadronization of a $b$-quark. This reconstruction happens offline, which means that events can be reprocessed with updated software if necessary. This chapter describes the reconstruction algorithms used for the analysis presented in this thesis.

\section{$3.1 \quad$ Tracks}

The reconstruction of particle tracks is done using hits. Hits are places where a signal is registered, so where a particle might have traversed the SMT or CFT. To fit particle tracks using those hits, two pattern finding algorithms are used in parallel. Then, the track is fitted using a Kalman track fitter [37] and a quality cut is applied to select only promising track candidates.

The first track-finding algorithm uses a model with the surfaces of all active tracking detectors [38, 39]. It creates paths from the center of the detector outward. Such a path is viable if the first few encountered surfaces contain at least three hits on those surfaces, with at least one in the SMT. The hits form a track segment, which is extrapolated from the inside to the outside of the tracker. The extrapolation follows a helicoidal trajectory due to the magnetic field. Energy loss and multiple scattering in the detector material are also taken into account. When the extrapolation crosses a detector surface, a hit is sought within a certain distance. There are several scenarios:

- the search window of the tracking algorithm was covered by an active part of the detector, but no hit was registered. This is called a 'missing hit'; 
- there is a hit. The track is refitted including the hit if this does not increase the overall $\chi^{2}$ too much, otherwise the hit is dropped resulting in the missing-hit scenario;

- there are multiple hits in the layer that are acceptable. The algorithm uses all these options as new hypotheses in parallel.

Finally, the track is re-examined for its track quality, determined by the total $\chi^{2}$ and the number of missed hits along the track, where the probability of such a miss is taken into account. Also, if two tracks share four or more hits once the track is complete, the one with the highest $\chi^{2}$ contribution is dropped.

The other algorithm is adopted from a Hough transform [40, 41]. To first approximation the solenoidal magnetic field $(B)$ is homogeneous and the particle has no interaction with the detector, so the tracks will be circular in the transverse $(x, y)$ plane. We demand the track to have a small distance of closest approach $\left(d_{0}\right)$ to the interaction point. Then, the circle has only two parameters: the $\phi$ angle at which the track started from the interaction point, and the curvature $\rho \equiv R^{-1}=q B / p_{\mathrm{T}}$, with $R$ the radius of the circle and $q$ the charge of the particle. Each point in $x, y$ space corresponds to a curve in $\phi, \rho$ space. The hits coming from the same track give different curves which intersect at the same point. Bins of a $\phi, \rho$ histogram are filled along the line found for each hit. The curve has a certain width coming from uncertainties, for example because $d_{0}$ is not necessarily zero. Then, the track parameters can be found by identifying the peaks in the histogram. This is done by removing bins with 3 or less entries and using the remaining bins to form possible circles.

Next, the hits corresponding to the found circles are used to determine $\phi, \rho$ and $d_{0}$ more precisely. Also, the other parameters of the track are determined: $\eta$ and the $z$ position at the closest approach, $z_{0}$. This is done by minimizing the total $\chi^{2}$ between the track and the hits.

All the tracks found in either of the two algorithms are fitted using a Kalman track fitter. The possible scattering with the detector and interaction with the magnetic field are also taken into account. If a track is found by both algorithms, the duplicate with the best Kalman fit is retained.

\subsection{Track Quality using CFT Timing}

To reduce the number of fake tracks, a fake-track rejection procedure is being developed in $\mathrm{D} \varnothing$. It consists of a multivariate discriminant with a number of track-related variables, amongst them the number of (SMT/CFT) hits and the overall $\chi^{2}$ of the track fit 42. CFT timing information, which is available for Run IIb, might be another useful variable. In this section, a method to correct the timing using information about the CFT hits is discussed [43]. 


\subsubsection{Overview}

First, the components contributing to the recorded time of a hit are discussed. A theoretical model of the most important component is explained in Section 3.2 .3 . Afterwards, this component is estimated using a data-driven approach, giving the expected time for a hit when the hit parameters are known.

The compatibility of the expected time with the observed time is then checked per hit, and combined along the track.

The aim of this study is to correct for physics effects, which could be cluttered by detector noise and software deficiencies. Therefore, strict track cuts are applied:

- $p_{\mathrm{T}}>1.5 \mathrm{GeV}$;

- $|\eta|<2.5$;

- $d_{0}<0.3 \mathrm{~cm}$ with an uncertainty smaller than $0.004 \mathrm{~cm}$;

- $\left|z_{0}\right|<5 \mathrm{~cm}$ with an uncertainty smaller than $0.2 \mathrm{~cm}$;

- \#SMT hits $\geq 3$; and

- \#CFT hits $>15$.

\subsubsection{Basic Chronology of one Fiber Hit}

At a certain time $t_{c}^{\prime}$, a collision produces a particle which travels through the inner detector. After a time of flight $t_{\mathrm{s}}$, the particle hits a CFT fiber (see Section 2.2.2 on pages 28 to 29 where it excites the fluorescent dye. The dye relaxes after some time, emitting photons which will be shifted to a lower frequency by absorption and emittance due to 3-hydroxyflavone molecules. Together, the relaxation time of the fluorescent and the waveshifter dyes is called $t_{\mathrm{r}}^{\prime}$.

The light will travel through the fibers which consist of a polystyrene core and two outside cladding layers with a lower refraction index. The photons can be reflected in one of the layers, depending on their initial position and angle, until they reach either the beginning of the waveguide, or the mirroring surface on the opposite side. In the latter case a photon will travel the length of the fiber again and reach the beginning of the waveguide afterwards. Since the waveguide is similar to the scintillating fibers, the distance through the waveguide to a VLPC also depends on the angle of emission of the photons. The time between emission and readout is called $t_{\mathrm{f}}^{\prime}$.

The VLPC registers photon signals by storing the time TDC and the signal height ADC. How long it will take the VLPC to recognize the signal and store the time depends on the signal height because higher signals pass the threshold more quickly. The time between the photon reaching the end of the waveguide fiber and the VLPC recognizing a signal is called $t_{\mathrm{ADC}}$. 
In summary, the time at which a hit is registered can be written as

$$
t_{\mathrm{reg}}=t_{\mathrm{c}}^{\prime}+t_{\mathrm{s}}+t_{\mathrm{r}}^{\prime}+t_{\mathrm{f}}^{\prime}+t_{\mathrm{ADC}}
$$

The time of flight of the particle, $t_{\mathrm{s}}$, can be computed as the length $s$ along its track from the collision point to the place where the particle hits the fiber, divided by the speed of the particle, so

$$
t_{\mathrm{s}}=\frac{s}{v} \text {. }
$$

The minimal momentum of a particle track is $0.5 \mathrm{GeV}$, making $t_{\mathrm{s}} \approx \frac{s}{c}$ a good approximation. ${ }^{1}$ A typical value for $t_{\mathrm{s}}$ is about $2 \mathrm{~ns}$.

The time it takes the signal to travel through the fiber, $t_{\mathrm{f}}^{\prime}$, depends on the place of the hit's $z$ coordinate. The number of emitted photons also influences $t_{\mathrm{f}}^{\prime}$, since the first photon arriving will determine the recorded time; if there are many photons, there is a large probability that at least one will go fairly straight towards the readout. The number of emitted photons can be estimated by the light yield $Y$, which is the number of photo-electrons measured at the readout. The light yield can be calculated with the measured ADC count by

$$
Y=\frac{\mathrm{ADC}-\text { pedestal }}{\text { gain }}
$$

where the pedestal and gain are variables of the each channel. An example of the $\mathrm{ADC}$ count and light yield distributions can be found in Figure 3.1 .

$t_{\mathrm{ADC}}$ depends on the pulse height, which is linear with the light yield for each individual fiber. Therefore, a data derived prediction for $t_{\mathrm{f}}^{\prime}$ will also include the light yield dependence of $t_{\mathrm{ADC}}$ and it is convenient to define $t_{\mathrm{f}} \equiv t_{\mathrm{f}}^{\prime}+t_{\mathrm{ADC}}$.

The relaxation time $t_{\mathrm{r}}^{\prime}$ is different for every photon and remains an unknown. The average decay time is about $7 \mathrm{~ns}$ [44].

We do not have a measure for the collision time $t_{c}^{\prime}$, but it should be equal for all hits on tracks associated with the same collision. Therefore our best estimate of $t_{\mathrm{c}}^{\prime}$ is the observed time minus its known contributions ( $t_{\mathrm{s}}$ and $t_{\mathrm{f}}$ ), averaged over all fibers of the track. This method will also consider the average $t_{\mathrm{r}}^{\prime}$ as part of $t_{\mathrm{c}}^{\prime}$, so we define $t_{\mathrm{c}} \equiv t_{\mathrm{c}}^{\prime}+\left\langle t_{\mathrm{r}}^{\prime}\right\rangle$ and $t_{\mathrm{r}} \equiv t_{\mathrm{r}}^{\prime}-\left\langle t_{\mathrm{r}}^{\prime}\right\rangle$.

The time before a hit is registered is now

$$
t_{\mathrm{reg}}=t_{\mathrm{c}}+t_{\mathrm{s}}+t_{\mathrm{r}}+t_{\mathrm{f}}
$$

The actual observed time for a hit on a channel is determined by correcting the measured TDC with the slope and offset, which are variables of each channel:

$$
t_{\mathrm{obs}}=\mathrm{TDC} \cdot \text { slope }+ \text { offset. }
$$

\footnotetext{
${ }^{1}$ The bulk of the tracks are charged pions with a mass of $140 \mathrm{MeV}$.
} 


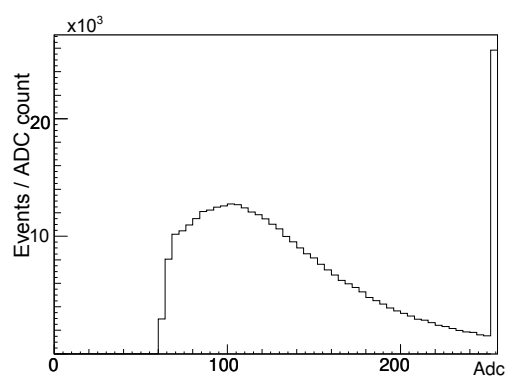

(a) ADC count.

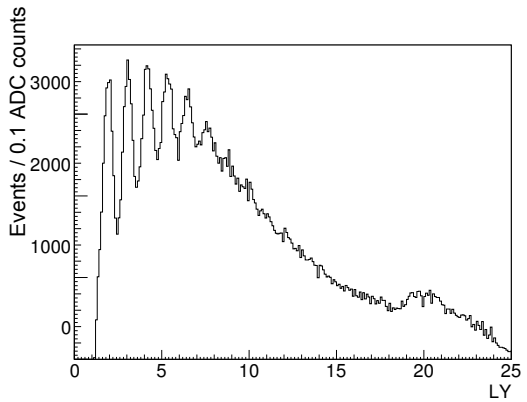

(b) light yield.

Figure 3.1: the distribution of (a) the ADC count and (b) the light yield of the outermost stereo layer. The ADC readout is capped at a maximum of 255, hence the overflow bin there. The structure at low light yields shows that individual photo-electrons can be distinguished. The bump at $Y \approx 20$ corresponds to the ADC overflow bin, spread out due to different channel gains and pedestals.

\section{Expected Time}

It is possible to construct an expected time before $t_{\mathrm{f}}$ is modeled. This is done by calculating $t_{\mathrm{s}}$ for every hit on a track and adding the average $t_{\mathrm{obs}}-t_{\mathrm{s}}$ of hits on the same track. This results in

$$
t_{\exp }^{\prime}=t_{\mathrm{s}}+t_{\mathrm{c}}+\left\langle t_{\mathrm{f}}\right\rangle
$$

The distribution of $t_{\mathrm{obs}}-t_{\mathrm{exp}}^{\prime}$ is shown in Figure 3.2 . The resolution at this point is $8.5 \mathrm{~ns}$.

The next step is to model $t_{\mathrm{f}}$ in order to get an optimal expected time with which the observed time of a specific hit can be compared.

\subsubsection{Theoretical Model of $t_{\mathrm{f}}$}

To understand the structure of $t_{\mathrm{f}}$, we can make a basic model. We approximate the scintillating fiber as a simple tube which is totally reflective on the outside and has no absorption. Suppose the length of the scintillating fiber is $2 L$, the waveguide has an additional length $L_{\mathrm{w}}$, and the index of refraction is $n$. The particle hits the fiber at $z \in[-L, L]$, where $z=L$ corresponds to the waveguide end of the scintillating fiber and $z=-L$ corresponds to the reflective end of the fiber. If one photon then starts at an angle $\theta$ with respect to the $z$ axis, the total 


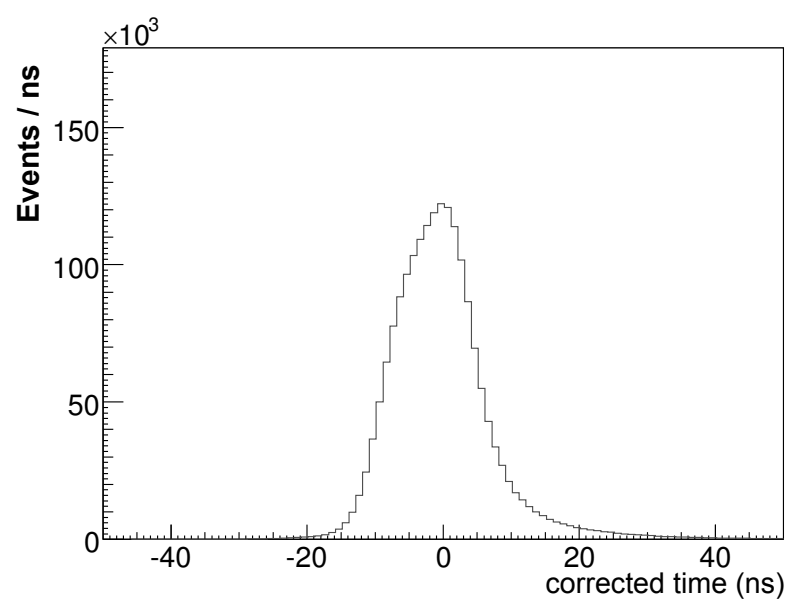

Figure 3.2: $t_{\mathrm{obs}}-t_{\exp }^{\prime}$ distribution for the outermost axial fiber layer.

time between the photon emission and registration is

$$
t= \begin{cases}n \frac{(L-z)+L_{\mathrm{w}}}{c \cos (\theta)} \equiv \frac{t_{1}}{\cos (\theta)} & \text { if } \cos (\theta)>0 \\ -n \frac{(3 L+z)+L_{\mathrm{w}}}{c \cos (\theta)} \equiv \frac{-t_{2}}{\cos (\theta)} & \text { if } \cos (\theta)<0\end{cases}
$$

where $t_{1}$ and $t_{2}$ depend on $z$ and represent the earliest arrival times possible for respectively a direct or a reflected photon.

We assume that the distribution of photons emitted from the waveshifter dye is isotropic in solid angle. If the photon starts with $\cos (\theta)>0$, so in the hemisphere on the read-out side, the probability that it is observed before time $t$ is given by

$$
p_{\cos (\theta)>0}(t) \equiv p\left(t_{\mathrm{obs}}<t\right)=p\left(\theta\left(t_{\mathrm{obs}}\right)<\theta(t)\right)=1-\cos (\theta(t)) .
$$

Using Equation (3.7) for $\cos (\theta)>0$ yields

$$
p_{\cos (\theta)>0}(t)=1-\frac{t_{1}}{t} .
$$

In the other hemisphere, the photon first travels to the reflecting end of the fiber and $p_{\cos (\theta)<0}(t)$ becomes

$$
p_{\cos (\theta)<0}(t)=1+\cos (\theta(t))=1-\frac{t_{2}}{t},
$$

where we used Equation 3.7 for $\cos (\theta)<0$. 
Equations (3.9) and (3.10) are only valid for $t>t_{1}$ and $t>t_{2}$, respectively. The probability that the photon is observed before these times is zero. Therefore, the combined probability that the photon was observed before time $t$ is

$$
p(t)=\left(\frac{1}{2}-\frac{t_{1}}{2 t}\right) H\left(t-t_{1}\right)+\left(\frac{1}{2}-\frac{t_{2}}{2 t}\right) H\left(t-t_{2}\right),
$$

with $H(x)$ the Heaviside function.

Suppose the number of emitted photons is $N$ and the signal was recorded if at least one photon has arrived at the end of the waveguide. The probability that this happens before time $t$ is equal to

$$
p(t \mid N)=1-(1-p(t))^{N}= \begin{cases}1-(1-0)^{N}=0 & \text { if } t<t_{1} \\ 1-\left(\frac{1}{2}+\frac{t_{1}}{2 t}\right)^{N}=1-\frac{\left(t+t_{1}\right)^{N}}{2^{N} t^{N}} & \text { if } t_{1}<t<t_{2} \\ 1-\left(\frac{t_{1}}{2 t}+\frac{t_{2}}{2 t}\right)^{N}=1-\frac{\left(t_{1}+t_{2}\right)^{N}}{2^{N} t^{N}} & \text { if } t_{2}<t,\end{cases}
$$

which gives

$$
p(t \mid N)=\left(1-\frac{\left(t+t_{1}\right)^{N}}{2^{N} t^{N}}\right) H_{2}+\left(1-\frac{\left(t_{1}+t_{2}\right)^{N}}{2^{N} t^{N}}\right) H_{3}
$$

where $H_{2} \equiv H\left(t-t_{1}\right)-H\left(t-t_{2}\right)$ and $H_{3} \equiv H\left(t-t_{2}\right)$ are defined for simplicity.

The probability density function (pdf) of the time that the first of $N$ photons arrives at the readout is

$$
f^{\prime}(t \mid N)=\frac{\mathrm{d} p(t \mid N)}{\mathrm{d} t}=\frac{N}{2^{N} t^{N+1}}\left[t_{1}\left(t+t_{1}\right)^{N-1} H_{2}+\left(t_{1}+t_{2}\right)^{N} H_{3}\right] .
$$

This distribution is smeared by the residual relaxation time $t_{\mathrm{r}}$. This is done by convoluting $f^{\prime}(t, N)$ with a Gaussian with width $\sigma$. The result is a total probability density distribution in $t$ given the number of emitted photons $N$ of

$$
\begin{aligned}
f(t \mid N)=\frac{N}{\sigma \sqrt{2 \pi} 2^{N}}\left[\int_{t_{1}}^{t_{2}} \frac{t_{1}\left(t^{\prime}+t_{1}\right)^{N-1}}{t^{\prime N+1}} e^{-\frac{\left(t^{\prime}-t\right)^{2}}{2 \sigma^{2}}} \mathrm{~d} t^{\prime}\right. \\
\left.\quad+\int_{t_{2}}^{\infty} \frac{\left(t_{1}+t_{2}\right)^{N}}{t^{\prime N+1}} e^{-\frac{\left(t^{\prime}-t\right)^{2}}{2 \sigma^{2}}} \mathrm{~d} t^{\prime}\right],
\end{aligned}
$$

which depends on the position of the hit by $t_{1}(z)$ and $t_{2}(z)$.

The probability distributions of the arrival time depending on $z$ are given in Figure 3.3 for different numbers of photo-electrons. For this simulation $L$ was taken to be $1.25 \mathrm{~m}, L_{w}=6 \mathrm{~m}$, the index of refraction $n=1.59$ and the width of $t_{\mathrm{r}}$ was taken at $\sigma=3 \mathrm{~ns}^{2}$ In this figure the measured distributions depending on

\footnotetext{
${ }^{2}$ The $3 \mathrm{~ns}$ width of $t_{\mathrm{r}}$ was based on observations in data.
} 
$z$ for different light yields $Y$ are also shown. The actual number of photons was likely larger than the light yield indicates; some photons may not have registered in time, were absorbed by the medium or had a $\theta$ such that they were not reflected by the cladding. The right (low $t$ ) sides of the probability density functions show an expected linear dependence on $z$. At a low number of photons there is a clear structure where the forward and backward peaks are well separated when the hit was close to the readout. Near the mirroring surface there is just a single peak.

Now the nature of the $z$ and $Y$ dependence of $t_{\mathrm{f}}$ is understood, we can use it to compare the prediction to data. This can be done by storing the data distributions of Figure 3.3 for every $Y$ and bunch of 16 channels with the same waveguide length, but that would mean more than 1 billion data points would have to be stored. Unfortunately, it is very costly in computer time if every CFT hit needs to be compared to this immense database.

\subsubsection{Data-Derived Corrections}

Another method to get an approximation of $t_{\mathrm{f}}(z, Y)$ is to assume the time distribution of $t_{\mathrm{f}}$ has the same Gaussian shape for all $z$ and $Y$; just its mean value depends on $z$ and $Y$. This assumption does not hold for hits with low light yield, which are close to the readout end of the fiber. For those hits timing is less relevant, since the $t_{\mathrm{f}}$ distribution is very wide.

To get the $z$ and $Y$ dependence of the average $t_{\mathrm{f}}$, the $z$ dependence is modeled by a $6^{\text {th }}$ order polynomial for every light yield, resulting in

$$
t_{\mathrm{f}}(z, Y)=\sum_{i=0}^{6} p_{i}(Y) z^{i}
$$

where $p_{i}$ are the parameters of the polynomial.

The dependence of $p_{i}$ on the light yield is modeled as a $3^{\text {rd }}$ order polynomial, so

$$
p_{i}(Y)=\sum_{j=0}^{3} q_{i j} Y^{j},
$$

with $q_{i j}$ the fit parameters, giving

$$
t_{\mathrm{f}}(z, Y)=\sum_{i=0}^{6} \sum_{j=0}^{3} q_{i j} Y^{j} z^{i} .
$$

For every layer these fits are performed separately, giving a total of $7 \times 4 \times 16=$ 448 parameters.

As an example, Figure 3.4 shows the $z$ dependence of $t_{\mathrm{f}}$ for the outermost axial layer. 


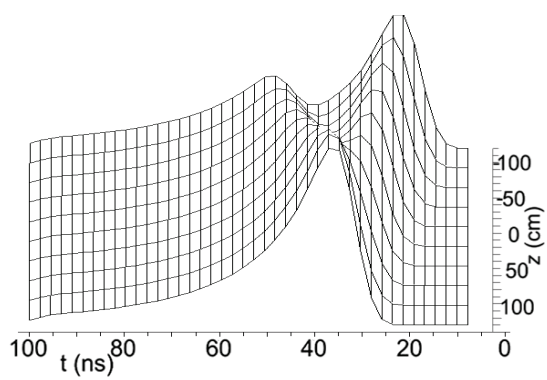

(a) $N=2$

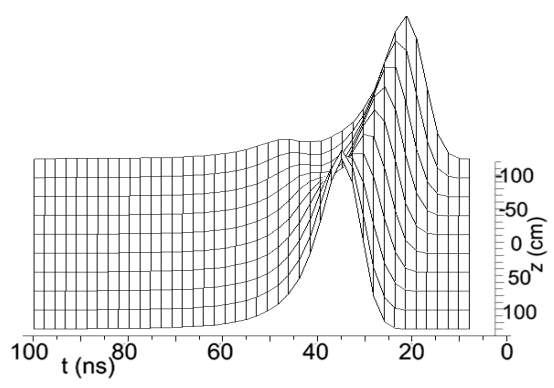

(c) $N=6$

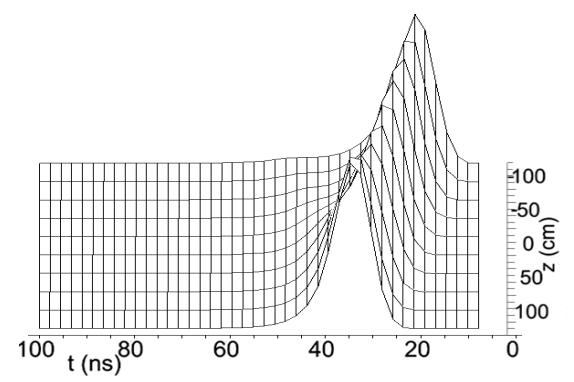

(e) $N=10$

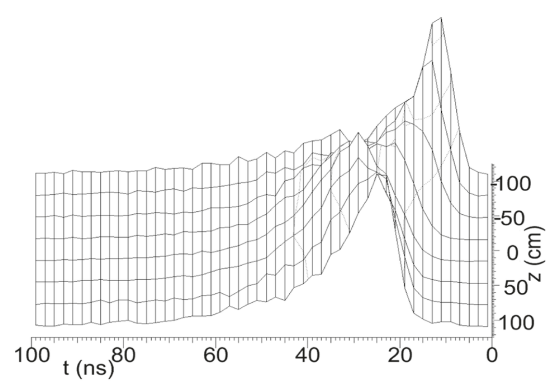

(b) $Y=2$

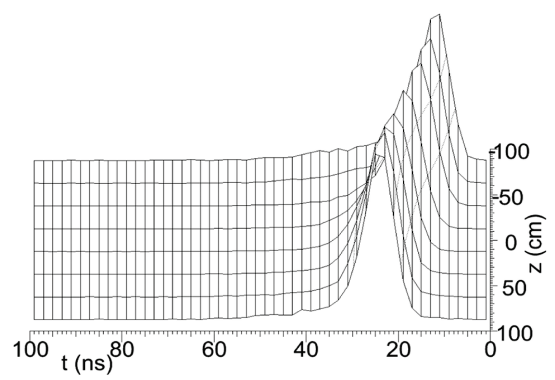

(d) $Y=6$

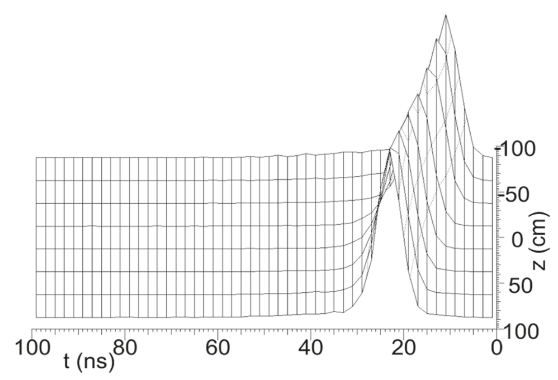

(f) $Y=10$

Figure 3.3: in (a), (c) and (e) the arrival-time probability density functions are plotted for different $z$ and number of photons. In (b) (d) and (f) the measured data distributions, normalized per $Y$ and $z$ bin are shown. 


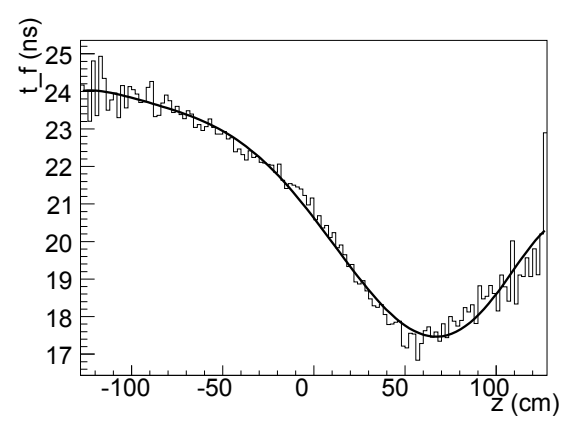

(a) $Y=6$.

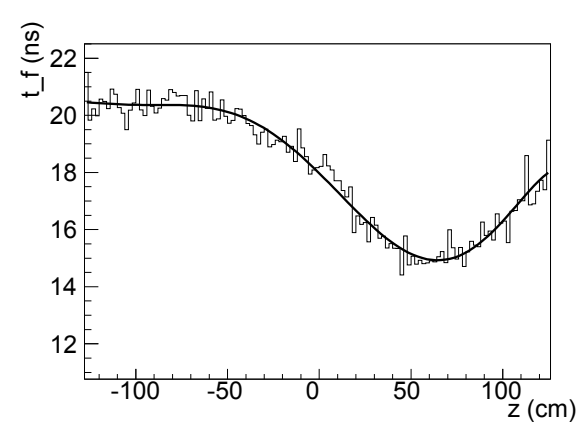

(b) $Y=14$.

Figure 3.4: the average $t_{\mathrm{f}}$ as a function of $z$ for two different light yield examples at the outermost axial fiber layer. The polynomial fits are also shown.

The dependence of $p_{i}$ on the light yield can be seen in Figure 3.5 . Especially at high $L Y$, the fits are not perfect. This could be explained by the bump observed in Figure $3.1(\mathrm{~b})$, which in turn is caused by the ADC overflow bin. Better modeling would only slightly improve the resolution, however.

We can now combine the result of $t_{\mathrm{f}}$ with our estimates for $t_{\mathrm{s}}$ and $t_{\mathrm{c}}$ to arrive at a final expected time; first $t_{\mathrm{s}}$ and $t_{\mathrm{f}}$ are determined from the track and hit properties, then the $t_{\mathrm{c}}$ is determined as the average $t_{\mathrm{obs}}-t_{\mathrm{s}}-t_{\mathrm{f}}$ per track. This results in

$$
t_{\exp }=t_{\mathrm{s}}+t_{\mathrm{f}}+t_{\mathrm{c}}
$$

An example of the resulting distribution for $t_{\mathrm{obs}}-t_{\exp }$ is shown in Figure 3.6. For comparison, $t_{\text {obs }}-t_{\text {exp }}^{\prime}$ from Figure 3.2 is also drawn.

The RMS of the distribution improves from 8.5 to $6.7 \mathrm{~ns}$, which is very comparable with the relaxation time of the 3 -hydroxyflavone. Therefore, the resolution is not expected to improve much with more sophisticated $t_{\mathrm{f}}$ predictions; the uncertainty on $t_{\mathrm{r}}$ will be dominant.

\subsubsection{Track Timing}

The timing information per hit can be combined to find the compatibility for an entire track. This is done by adding the $\chi^{2}$ per hit, divided by the number of hits on the track $n$ :

$$
\chi^{2}=\frac{1}{n} \sum_{i=1}^{n} \frac{\Delta t_{i}^{2}}{\sigma_{i}^{2}},
$$




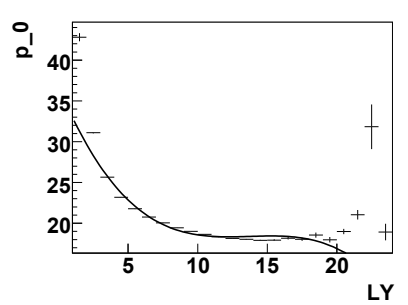

(a) parameter $p_{0}$

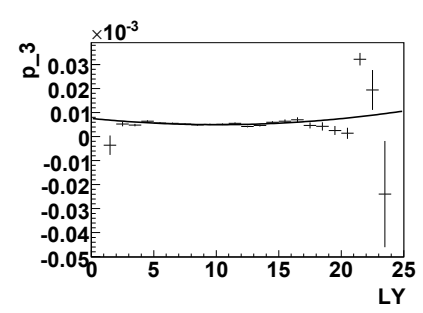

(d) parameter $p_{3}$

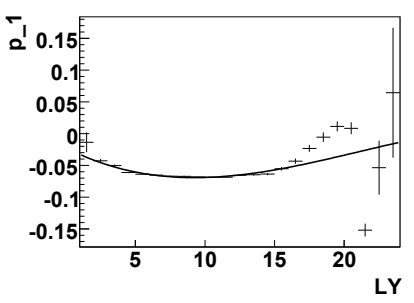

(b) parameter $p_{1}$

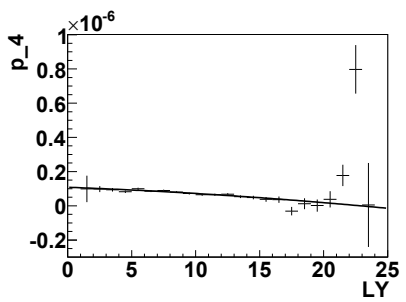

(e) parameter $p_{4}$

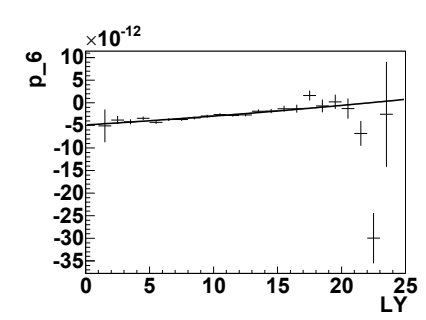

(g) parameter $p_{6}$

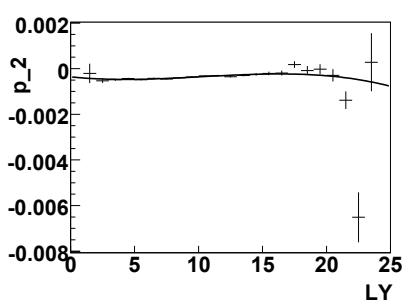

(c) parameter $p_{2}$

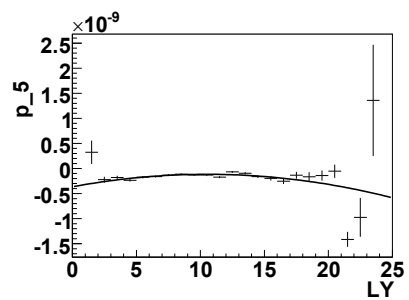

(f) parameter $p_{5}$ 


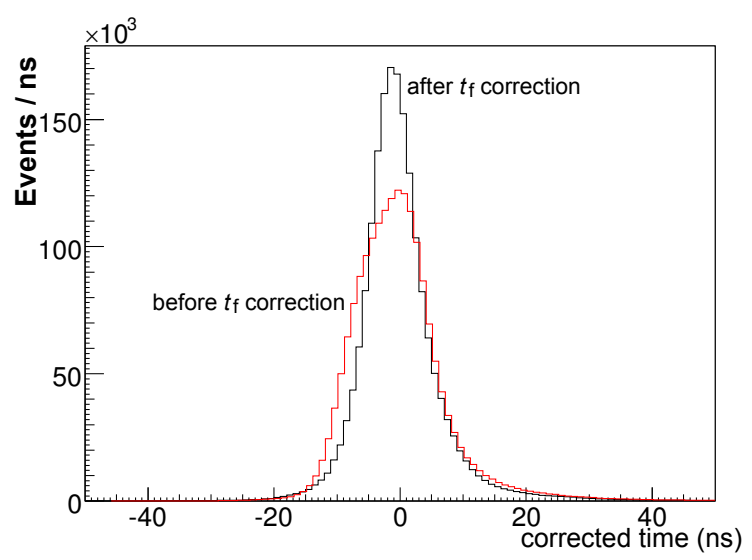

Figure 3.6: comparison between $t-t_{\exp }^{\prime}$ (red) and $t-t_{\exp }$ (black) for the outermost axial fiber layer.

where $\Delta t_{i}$ is $t_{\mathrm{obs}}-t_{\text {exp }}$ for hit $i$. The spread of the layer, $\sigma_{i}$, is taken to be the width of the corrected-time distributions, like the one in Figure 3.6

As a sanity check, Figure 3.7 shows the overall $\chi^{2}$ for about $2 \cdot 10^{5}$ tracks with the strict requirements listed in Section 3.2.1. The mean value of $\chi^{2}$ is found to be close to 1 , as expected, and no non-gaussian tails are observed.

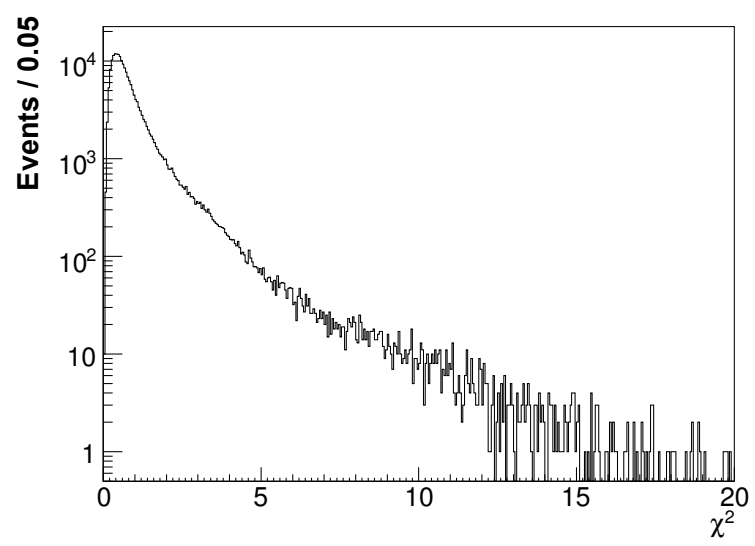

Figure 3.7: the $\chi^{2}$ per track. 


\subsubsection{Fake Track Suppression}

The CFT timing information can now be used to suppress fake tracks. The $\chi^{2}$ calculated per track can be a helpful additional input parameter to the Fake Track Discriminant described in [42.

Another way to use the CFT timing to suppress fake tracks is by rejecting fake hits before the pattern recognition [45]. If an ADC-dependent cut on the TDC is placed such that only $1 \%$ of good hits are removed from the pattern recognition input, many more fake hits are removed. In that case, the number of good tracks found actually increases by $2 \%$, while the number of fake tracks is reduced by $15 \%$. Because the number of hits used by the track finder decreases, so does the amount of computer time needed to perform the track reconstruction.

Both methods (the fake-track discriminant and the fake-hit rejector before the pattern recognition) were not used for the data reconstruction for the analysis described in Chapters 4 and 5 .

\subsection{Vertices}

Intersecting tracks can indicate a vertex: a point in space where several tracks originated. This can be a primary vertex (PV), where the high-energy interaction took place, or where less-interesting soft scattering happened due to multiple collisions in a bunch crossing. A vertex can also be a secondary vertex (SV), which is where the decay of a relatively long-lived particle took place. Secondary vertices can be identified by their displacement from the collision point. This requires the PV to be known first.

The PVs are found using an adaptive algorithm [46]. First, candidate tracks are selected by requiring $p_{\mathrm{T}}>0.5 \mathrm{GeV}$. PV candidates are formed from tracks with the requirement that the $z$ distance at closest approach between the PV and the track is less than $2 \mathrm{~cm}$. Then, the following two steps are repeated:

1. a vertex is fitted using the candidate tracks, with weights $w$. Initially, the weight for each track is set to unity;

2. with the new fitted PV, the weights of the tracks are determined from the $\Delta \chi^{2}$ they add to the fit:

$$
w=\frac{1}{1+e^{\frac{1}{2}\left(\Delta \chi^{2}-\chi_{1 / 2}^{2}\right)}},
$$

where $\chi_{1 / 2}^{2}$ is the cutoff where the weight is $1 / 2$. If a weight has a value below $10^{-6}$, the corresponding candidate track is no longer used for the fit.

The iteration stops if the weights have a difference between two steps smaller 
than $10^{-3}$. If this does not occur within 100 iterations, the algorithm is forced to conclude non-convergence.

The tracks belonging to the PV are removed from the list and the procedure is repeated to look for more PV candidates. The one with the highest average track $p_{\mathrm{T}}$ is selected as the $\mathrm{PV}$ of the hard scattering.

In Section 3.7 is discussed how SVs are found.

\subsection{Muons}

Multiple hits in each layer of the muon scintillators and drift chambers are combined into track segments using straight-line fits. A-layer segments are also required to be compatible with a muon coming from the primary interaction point: $z_{0}^{\mu}-z_{\mathrm{PV}}<2 \mathrm{~cm}$. Tracks are then formed by interpolating the track segments and combining them with segments or single hits of other layers. When the interpolation passes the toroid, so between the A and B layer, an opening angle of 0.6 radian is allowed between segments.

Then, the tracks are propagated inward and a matching central track is sought. If a good match is found, the accuracy of the central tracker ensures a precise muon $p_{\mathrm{T}}$ determination. The time recorded by the scintillators minus the time of flight is required to be within $10 \mathrm{~ns}$ from the time of the bunch crossing.

$\mathrm{D} \varnothing$ has several definitions for the quality of muons. For this analysis we veto on events with 'loose' muons in order to achieve orthogonality with the $W H \rightarrow \mu \nu b \bar{b}$ channel, as discussed further in Section 4.2.3. For those muons at least two out of the following three criteria are required:

- a track segment of at least two wire and one scintillator hit in the A layer of the muon system;

- a track segment of at least two wire and one scintillator hit in the B or C layer;

- a track in the central tracking system.

The satisfied criteria need to be compatible with the same track; the two track segments have to be matched, or the single track segment should be matched with the inner detector track.

For more information on the offline muon reconstruction, see [47, 48].

\subsection{Photons and Electrons}

Electron and photon identification have many similarities. First, a seed cell is found by requiring $E_{\mathrm{T}}>0.5 \mathrm{GeV}$ in the electromagnetic part of the calorimeter. 
Then, a cone of radius $R=0.2$ in the $\eta \times \phi$ plane is made around the cell, which is required to have $E_{\mathrm{T}}>1 \mathrm{GeV}$. A cone with $R=0.4$ is then taken as photon or electron candidate if it has:

- more than $90 \%$ of its energy in the EM calorimeter;

- a shower that is consistent in shape with an EM shower; and

- an isolated cone, meaning that less than $15 \%$ of the energy of the cone should be in the region $0.2<R<0.4$.

Candidates become photons unless they are vetoed because there is too much activity in the tracker, either because of a reconstructed track in $\Delta R<0.05$ from center of the cone, or if the hit density in that area is too high.

Electrons are candidates that have a track within $\Delta R=0.05$ from the cone's $E_{\mathrm{T}}$-weighted center. Furthermore, electrons are selected on the 'electron likelihood', which combines different calorimeter and tracking parameters in a multivariate discriminant [49].

Calorimetry objects in general have a relative energy resolution composed of noise $(N)$, shower interactions and signal sampling $(S)$ and calibration errors $(C)$ :

$$
\frac{\sigma_{E}}{E}=\frac{N}{E} \oplus \frac{S}{\sqrt{E}} \oplus C
$$

For electrons and photons, $N=0.20 \mathrm{GeV}, S=0.26 \sqrt{\mathrm{GeV}}$ and $C=0.005$ [50].

In the analysis described in this thesis, a veto on electrons is placed, as discussed in Section 4.2.3.

See [51] for more details on photon and electron identification.

\subsection{Jets}

Jets are a result of the hadronization of quarks or gluons. By measuring jets, the kinematic properties $(E, \eta, \phi)$ of these partons can be approximated, assuming parton-hadron duality.

\subsubsection{Calorimeter Noise Reduction}

To reduce the impact of calorimeter noise on the jets, isolated noise-like cells are discarded. For each cell the RMS (root mean square) of the energy is determined, as a measure of its variation. Cells are ignored if they have a signal less than 4 times RMS above pedestal. If a certain cell did pass this threshold, the criteria for the neighboring cells in 3 dimensions are reduced to 2 times RMS. The cells are then combined into calorimeter towers, as discussed in Section 2.2.3.

The number of cells rejected by the noise reduction is between 30 and $60 \%$ in each event 52 . 


\subsubsection{Jet Finding Algorithm}

First, 'protojets' are formed using the Improved Legacy Cone Algorithm (ILCA) for clustering [53]; seeds are made from all calorimeter towers with $E_{\mathrm{T}}>1 \mathrm{GeV}$. Around them, a cone with radius $R=0.5$ in the $\eta \times \phi$ plane is formed. The cone's $E_{\mathrm{T}}$-weighted center is determined and used as a new seed. This continues until the cone is stable, at which point it is known as a protojet.

The next step is to split or merge the protojets. They are ordered in $E_{\mathrm{T}}$ and starting with the highest- $E_{\mathrm{T}}$ jet, overlapping regions with other jets are found. Two jets are merged if the $E_{\mathrm{T}}$ in the shared calorimeter towers is larger than half of the $E_{\mathrm{T}}$ of the lowest-energy jet. If not, each tower is assigned to the jet closest to it.

After the merging or splitting, the ILCA recalculates the weighted center(s) of the altered jet(s). This is repeated until there are no overlapping jets anymore.

Finally, the following additional cuts are applied to accept a jet:

- the $E_{\mathrm{T}}$ of a jet has to be larger than $8 \mathrm{GeV}$;

- the trigger information should be consistent with a jet. This reduces the effect of spurious noise coming from the precise readout chain, which is not used in triggering;

- the electromagnetic fraction of the energy $\left(f_{\mathrm{EM}}\right)$ has to be larger than $5 \%$ and smaller than $95 \%$ to cut away hadronic calorimeter noise and electrons or photons, respectively;

- less than $40 \%$ of the jet's energy should be in the coarse hadronic calorimeter, because the noise levels there are higher, due to the larger size of the cells;

- the highest-energy cell in the jet is required to be less than 10 times higher than the cell with the next-to-highest energy, because some hot cells can cause the formation of a fake jet that is dominated by a single cell.

\section{Infrared and Collinear Safety}

Because QCD has a large coupling constant at low energy scales, new particles are created with a probability close to unity if they have hardly any momentum in either the center-of-mass frame, or relative to one of the other colored particles. The jet algorithm should be insensitive to these effects: it should be infrared and collinear safe.

Infrared safety is achieved by also taking 'midpoints' between two jets as a seed for a new one. These jet pairs have to be less than $R=1$ apart, because otherwise the centers of the jets could not fit in a single cone anyway. Figure 3.8(a) shows this pictorially. These midpoint seeds are included in the procedure described 

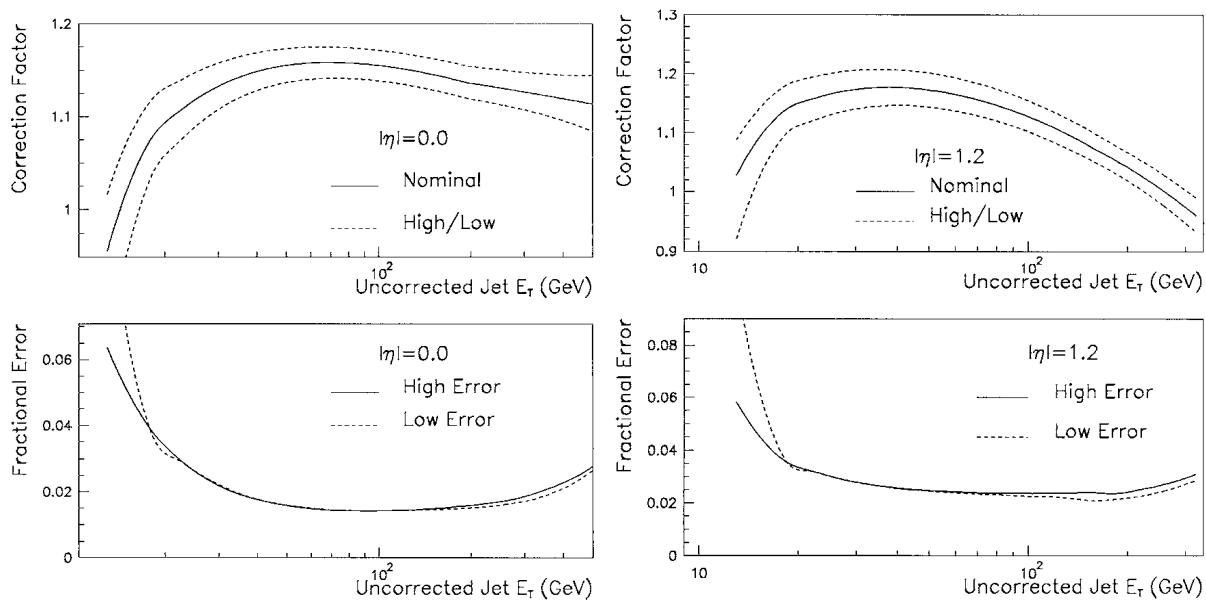

Figure 3.9: The magnitude of the JES correction and its uncertainty as a function of jet energy for $|\eta|=0$ and $|\eta|=1.2$ [54].

a combination of $\mathrm{MC}$ and data; $\mathrm{MC}$ is used to determine how much energy of the original parton escapes the cone during the fragmentation process. In data events, the amount of energy leaking outside of the cone due to showering in the calorimeter is determined by measuring the energy in rings (up to $R=1.0$ ) around the cone.

The corrected combined energy of the particles in the jet is determined by

$$
E=\frac{E_{\text {measured }}-O}{S \cdot R_{\text {det }}} .
$$

The remaining (unknown) biases in the method of determining the Jet Energy Scale (JES) are treated as a systematic uncertainty.

The total correction and uncertainty is displayed in Figure 3.9

\subsubsection{Jet Energy Resolution}

The resolution of the jet energy is determined by measuring the asymmetry in events with two back-to-back jets for every $\eta$ bin [55]. The asymmetry,

$$
A \equiv \frac{E_{\mathrm{T}^{\text {jet } 1}}-E_{\mathrm{T}^{\text {jet2 }}}}{E_{\mathrm{T}^{\text {jet } 1}}+E_{\mathrm{T}^{\text {jet2 }}}},
$$

is centered around zero, with a width of $\sigma_{A}$. The relative energy uncertainty is related to $\sigma_{A}$ as

$$
\frac{\sigma_{E_{\mathrm{T}}}}{E_{\mathrm{T}}}=\sqrt{2} \sigma_{A}
$$


which is a function of jet energy through Equation (3.21). For each $\eta$ bin, the $\sigma_{E_{\mathrm{T}}}\left(E_{\mathrm{T}}\right)$ is fitted to determine $N, S$ and $C$. Typical values are $N=3 \mathrm{GeV}$, $S=0.9 \sqrt{\mathrm{GeV}}$ and $C=0.05$.

Like for the JES, a systematic uncertainty is used to account for remaining biases in the Jet Energy Resolution (JER) determination.

\subsubsection{Corrections to Monte Carlo Jets}

Monte Carlo generated jets do not have many of the detector effects mentioned before. Therefore, the Jet Shifting, Smearing and Removing (JSSR) procedure was introduced, to have the behavior of $\mathrm{MC}$ jets better mimic that of data jets [56]. To this end, the relative difference between the jet and the photon energy,

$$
\Delta S=\frac{E_{\mathrm{T}}^{\mathrm{jet}}-E_{\mathrm{T}}^{\gamma}}{E_{\mathrm{T}}^{\gamma}},
$$

was measured in $\gamma+$ jet data and MC samples. The difference between the mean $\Delta S$ in data and the mean $\Delta S$ in MC was used to shift the energy of the simulated jets randomly.

The smearing was determined by the difference in the widths:

$$
\sigma_{\text {correction }}^{2} \equiv \sigma_{\Delta S, \text { data }}^{2}-\sigma_{\Delta S, \mathrm{MC}}^{2}
$$

Also, the MC jet-identification efficiency is equalized with data jet efficiencies by randomly removing jets with a probability equal to the difference in efficiency between $\mathrm{MC}$ and data. This is mainly important in the ICR region, where about $2 \%$ of $\mathrm{MC}$ jets is removed.

The shifting, smearing and removal of MC jets is performed as a function of jet $p_{\mathrm{T}}$ for the CC, ICD and EC regions separately. The difference between data and $\mathrm{MC}$ can be seen in Figure 3.10 [56].

\section{$3.7 \quad b$-Jet Identification}

The identification of $b$-jets is very important for this analysis, because the Higgs boson mainly decays into $b$ 's if it has a low mass, as explained in Section 1.2.2. The main distinction between a $b$-jet and a light-flavored jet is a secondary vertex; $b$-quarks form hadrons that have a lifetime long enough such that they propagate a detectable distance (in the order of a $\mathrm{mm}$ ) before they decay. Therefore, to identify a jet as a $b$-jet, one can look for indications of a displaced vertex: high impact parameter tracks or a SV.

Candidate $b$-jets have at least two tracks in the $R=0.5$ cone. The tracks should each have at least two SMT hits and $p_{\mathrm{T}}>0.5$. Then, that jet is called 'taggable', meaning it can be considered for $b$-tagging. 


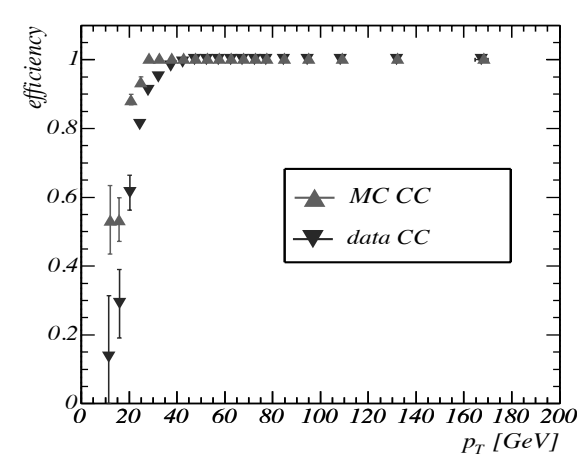

(a)

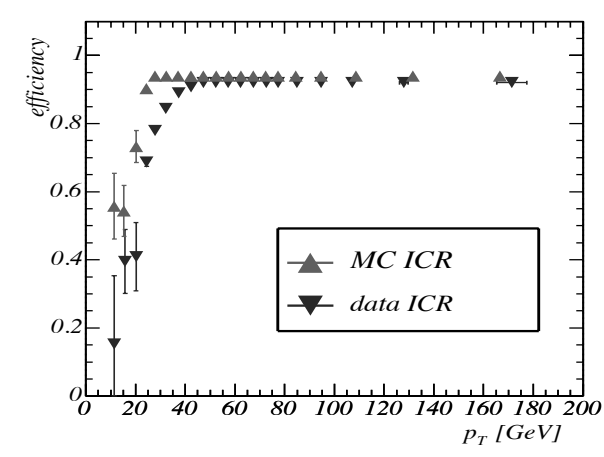

(b)

Figure 3.10: jet-identification efficiencies in data and $\mathrm{MC}$ as a function of $p_{\mathrm{T}}$ in (a) the CC and (b) the ICR region of the calorimeter.

Next, indications of a secondary vertex are sought, which is done using a Kalman Filter on tracks that are not associated with the PV [57]. Three different algorithms determine the likelihood of a SV belonging to a $b$-decay. These algorithms are:

- the Counting Signed Impact Parameter (CSIP) tagger, which determines the number of tracks within a large signed ${ }^{3}$ impact parameter significance $d_{0}^{\text {sig }}=d_{0} / \sigma_{d_{0}}$ [58, 59];

- the Jet Lifetime Impact Parameter (JLIP) tagger, where the probability is calculated that all tracks in the jet originated from the PV [60, 61]; and

- the Secondary Vertex Tagger (SVT), which demands a decay length significance greater than 3 for at least one of the secondary vertices of the jet 62 .

The results of the algorithms are combined using a neural network (NN), which is trained to distinguish $b$-jets from other jets [63. Ordered by importance, the input variables of the $\mathrm{NN}$ are:

1. the decay length significance of the SV, where the decay length is the distance between the secondary and primary vertices; ${ }^{4}$

2. a weighted combination of CSIP's $d_{0}^{\text {sig }}$ results $;^{5}$

\footnotetext{
${ }^{3}$ The track's point of closest approach to the PV is projected on the jet momentum. The sign of this projection is taken as the sign of the impact parameter.

${ }^{4}$ If there are multiple SVs, the one with the highest decay-length significance is chosen.

${ }^{5}$ The combination is given by $6 s_{3}+4 s_{2}+3 w_{3}+2 w_{2}$, where $s_{2}\left(s_{3}\right)$ is the number of tracks
} 

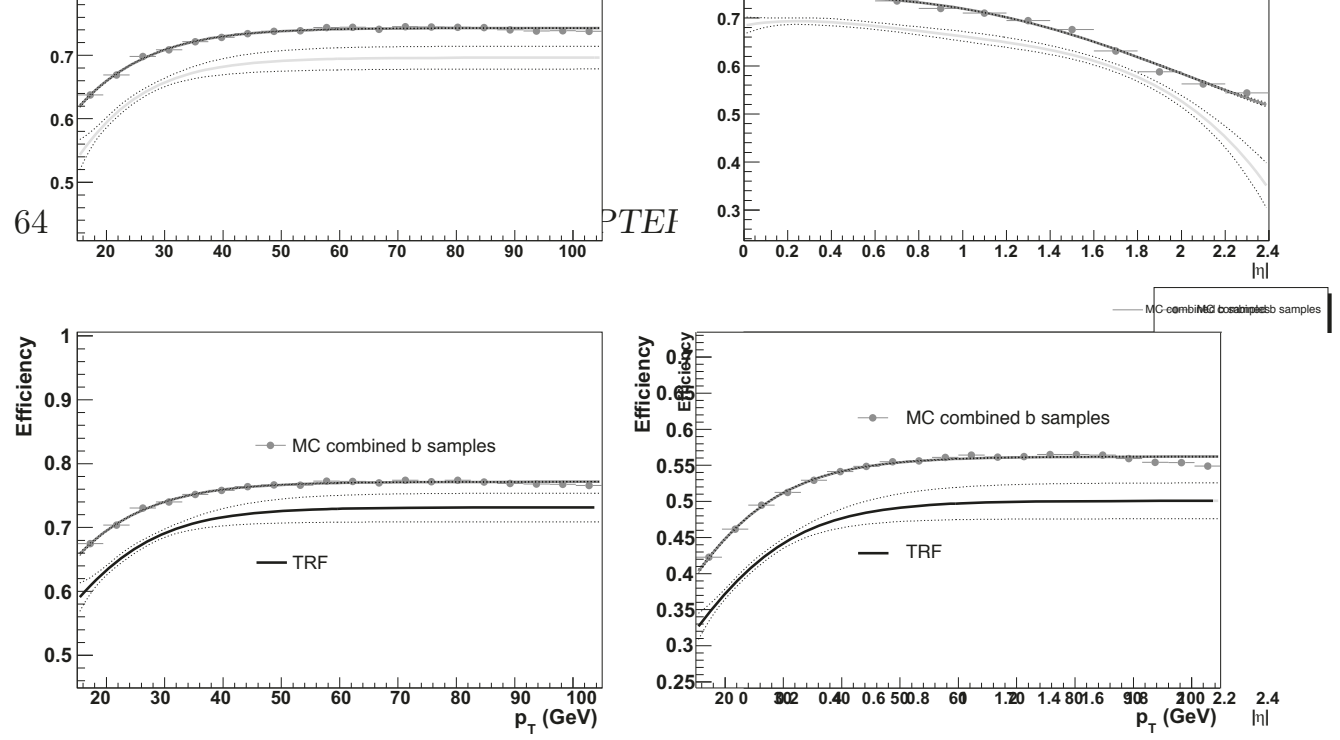

(a) operating point 'Loose'

(b) operating point 'Tight'

Figure 14. NN tager $b$-iet efficiengy as a fungtion of $p$ (deft) and 9 (right) in the combined $b$-jet

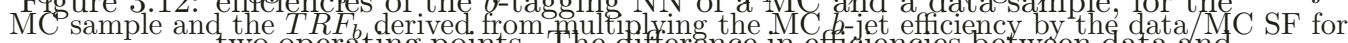

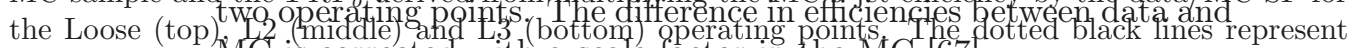

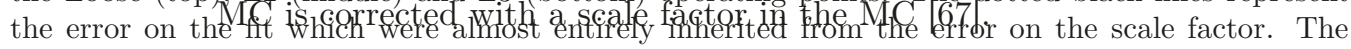
functions used for the parameterisation are outlined in the text.

light jet events in each sample render four unknowns, and the efficiencies of the taggers for the different jet types add another four unknowns. The latter include the parameters of interest: the TRF and NTR. Coefficients derived from MC are used to account for correlations, for example between the two taggers [67.

The fake rate is on average about $5 \%$ and $0.5 \%$ for the Loose and Tight point, respectively 68 .

Jets with $c$-hadrons are also tagged frequently, albeit with a lower efficiency than $b$-jets. Separate TRFs are determined for these $c$-jets. It is assumed that the scale factor between MC prediction and data is the same for $b$ - and $c$-jets [64].

\subsection{Tau Identification}

The $\tau$ is hard to identify. About a third of taus decays leptonically and the rest decays hadronically, as can be seen in Table 3.1. The one difference between leptonic $\tau$ decays and direct $e$ or $\mu$ production is in the accompanying neutrinos, which can only be detected by their contribution to the $\mathbb{E}_{\mathrm{T}}$. Therefore, leptonic $\tau$ decays are treated as part of the $e$ and $\mu$ channels, $W H \rightarrow \mu \nu b \bar{b}$ and $W H \rightarrow e \nu b \bar{b}$, as $W H \rightarrow \tau \nu b \bar{b} \rightarrow e \nu \bar{\nu} b \bar{b}$ and $W H \rightarrow \tau \nu b \bar{b} \rightarrow \mu \nu \bar{\nu} b \bar{b}$.

Hadronic tau decays can appear to be similar to jets from hadronization. Compared to the secondary vertices of $b$-quarks, those of $\tau$ decays are harder to measure: the $\tau$ decay length is similar or shorter and its decay products have tracks nearly collinear to the original $\tau$ direction due to its high boost, making them less distinguishable from PV tracks. Also, the decay products often include 
only one charged particle, making SV finding more difficult.

Despite these difficulties, a $\tau$-identification algorithm has been developed. It consists of a candidate-selection procedure, the separation of three $\tau$ types and a neural network.

Tau candidates are identified by finding a calorimeter cluster, after which associated tracks and EM sub-clusters (EMSCs) are sought.

Calorimeter clusters are found by a simple cone algorithm. They have an $R=0.5$ cone and a core cone of $R=0.3$. Isolation is enforced by requiring that more than half of the energy in the larger cone lies within the core cone.

Associated tracks are likely to have been produced in the $\tau$ decay. The tracks can become associated with the $\tau$ in four consecutive steps 69]:

1. all tracks with $p_{\mathrm{T}}>1.5 \mathrm{GeV}$ and within the $R=0.5$ cone are ordered by decreasing $p_{\mathrm{T}}$;

2. up to 2 tracks within $\Delta z_{0}=2 \mathrm{~cm}$ relative to the first track's $z_{0}$ are examined;

3. a second track is added if the combined invariant mass with the first track is lower than $1.1 \mathrm{GeV}$;

4. a third track is added if the combined invariant mass with the first two tracks is lower than $1.7 \mathrm{GeV}$ and the charges of the three tracks add up to \pm 1 .

EM sub-clusters can be $\pi^{0}$ s from the $\tau$ decay. They are formed using a nearest neighbor algorithm in the third, most finely grained layer of the EM calorimeter (EM3) [70]. These EM sub-clusters have a minimum energy of $800 \mathrm{MeV}$. If a subcluster is found, other EM layers (EM12) and preshower hits are included.

\begin{tabular}{ll}
\hline$\tau^{-}$decay mode & branching ratio \\
\hline$\pi^{-} \pi^{0} \nu_{\tau}$ & $0.2551 \pm 0.0009$ \\
$e^{-} \overline{\nu_{e}} \nu_{\tau}$ & $0.1785 \pm 0.0005$ \\
$\mu^{-} \nu_{\mu} \nu_{\tau}$ & $0.1736 \pm 0.0005$ \\
$\pi^{-} \nu_{\tau}$ & $0.1091 \pm 0.0007$ \\
$\pi^{-} \pi^{0} \pi^{0} \nu_{\tau}$ & $0.0929 \pm 0.0011$ \\
$\pi^{-} \pi^{+} \pi^{-} \nu_{\tau}$ & $0.0903 \pm 0.0006$ \\
all other modes & 0.1005 \\
\hline
\end{tabular}

Table 3.1: branching ratios of the dominant $\tau^{-}$decay processes [6]. The decay modes of $\tau^{+}$are the antimatter equivalents. 


\begin{tabular}{llllll}
\hline Variable & Definition & 1 & 2 & 3 & $\mathrm{NN}_{e}$ \\
\hline profile & $E_{\mathrm{T}}^{\text {leading } 2 \text { towers }} / E_{\mathrm{T}}$ & $\mathrm{X}$ & $\mathrm{X}$ & $\mathrm{X}$ & $\mathrm{X}$ \\
calo. isolation & $\left(E_{\mathrm{T}}-E_{\mathrm{T}}^{R<0.3}\right) / E_{\mathrm{T}}^{R<0.3}$ & $\mathrm{X}$ & $\mathrm{X}$ & $\mathrm{X}$ & \\
track isolation & $p_{\mathrm{T}}^{\text {not ass. tracks }} / p_{\mathrm{T}}^{\text {ass. tracks }}$ & $\mathrm{X}$ & $\mathrm{X}$ & $\mathrm{X}$ & \\
EM12 fraction & $E_{\mathrm{T}}^{\text {EM12 } / E_{\mathrm{T}}}$ & $\mathrm{X}$ & & & \\
$\delta \alpha$ & angle between ass. tracks and EMSCs & & $\mathrm{X}$ & $\mathrm{X}$ & $\mathrm{X}$ \\
rms & width of the calorimeter cluster & $\mathrm{X}$ & $\mathrm{X}$ & $\mathrm{X}$ & $\mathrm{X}$ \\
f.h.f. & $E_{\mathrm{T}}^{\text {fine hadronic }} / E_{\mathrm{T}}$ & $\mathrm{X}$ & $\mathrm{X}$ & & \\
EMSC fraction & $E_{\mathrm{T}}^{\mathrm{EMSCs}} / E_{\mathrm{T}}$ & & $\mathrm{X}$ & $\mathrm{X}$ & $\mathrm{X}$ \\
profile3 & $E_{\mathrm{T}}^{\text {leading EMSC }} / E_{\mathrm{T}}^{\mathrm{EM} 3}$ & & $\mathrm{X}$ & $\mathrm{X}$ & \\
$p_{\mathrm{T}}$ fraction & $E_{\mathrm{T}} /\left(E_{\mathrm{T}}+p_{\mathrm{T}}^{\text {ass. tracks }}\right)$ & & $\mathrm{X}$ & $\mathrm{X}$ & $\mathrm{X}$ \\
\hline
\end{tabular}

Table 3.2: the input variables of the neural networks. An ' $\mathrm{X}$ ' indicates the variable is used for the $\mathrm{NN}$ of this tau type or for the $\mathrm{NN}_{e}$.

Because different hadronic tau decays give different detector signatures, the candidates are divided into three types of taus:

Type 1, a calorimeter cluster with exactly one associated track and no EM subclusters. These taus correspond to the $\pi^{ \pm} \nu_{\tau}$ decay;

Type 2, a calorimeter cluster with exactly one associated track and at least one EM sub-cluster. Type 2 corresponds mainly to the $\pi^{ \pm} \pi^{0} \nu_{\tau}$ decay, but it can also be an electron faking a tau;

Type 3, a calorimeter cluster with at least two associated tracks. This type corresponds mainly to the $\pi^{-} \pi^{+} \pi^{-} \nu_{\tau}$ decay.

For every tau type, a neural network $\left(\mathrm{NN}_{\tau}\right)$ is trained, using variables connected to the shower shape, the isolation and the correlation between calorimeter and tracks. The variables are listed in Table 3.2 . Because many type 2 taus are in reality electrons, also a $\mathrm{NN}$ was trained to separate type 2 taus from electrons, $\mathrm{NN}_{e}$. For more information on the tau neural networks, see [71].

In the $W H \rightarrow \tau \nu b \bar{b}$ analysis, only the first two tau types are used, because type 3 taus have a higher QCD background.

The output distributions of the $\mathrm{NN}_{\tau}$ for type 1 and 2 taus are shown in

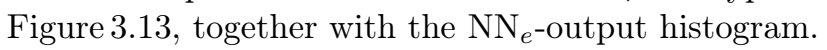

The tau candidates are corrected in $\mathrm{MC}$ for both the $\mathrm{NN}_{\tau}$ and track efficiency. These factors are less than $5 \%$ in magnitude and are documented in [73. 


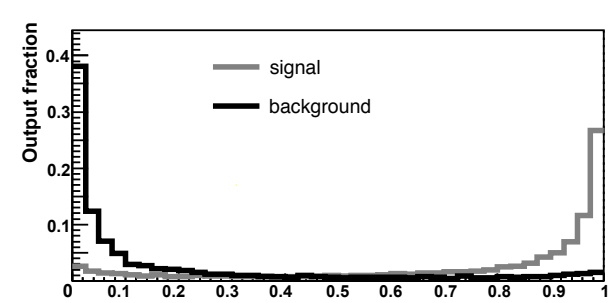

(a) output of the tau type $1 \mathrm{NN}_{\tau}$

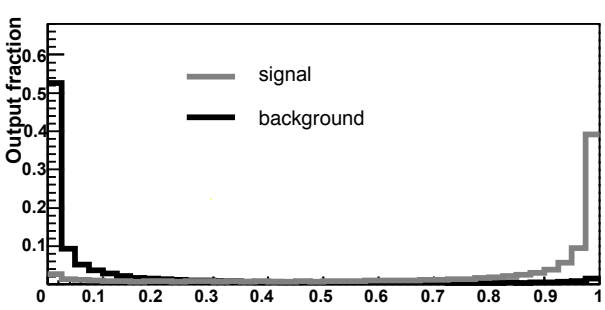

(b) output of the tau type $2 \mathrm{NN}_{\tau}$

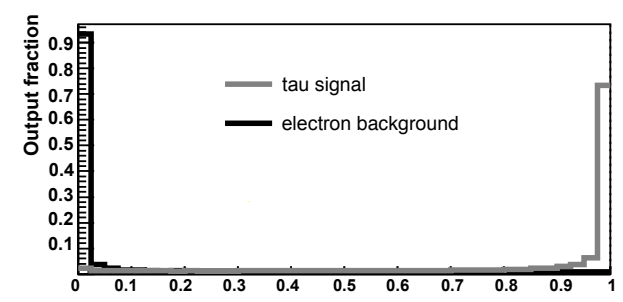

(c) output of the $\mathrm{NN}_{e}$

Figure 3.13: histograms of the output variables for the tau type 1 and 2 neural networks [72].

\subsection{Missing Transverse Energy}

Neutrinos have a negligible chance of interacting with the detector in any way. This means that energy can be carried away without being seen, causing an energy imbalance. As mentioned in Section 2.2.1, the intrinsic nature of hadron collisions prevents us from identifying a longitudinal imbalance, so only the transverse energy of the neutrinos can be determined.

We are mainly interested in the neutrino involved in the hard scattering, but if more than one neutrino is present, only the sum of the neutrino $\vec{p}_{\mathrm{T}}$ is known. Therefore, the primary-neutrino $E_{\mathrm{T}}$ has an intrinsic uncertainty due to neutrinos coming from subsequent decays and other decays in the event.

Establishing whether an event has missing $E_{\mathrm{T}}$ depends on the resolution of all objects carrying energy: jets, electrons, photons, muon tracks and unclustered transverse energy $\left(U_{\mathrm{T}}\right)$. Unclustered energy is energy in the calorimeter not assigned to jets or EM objects and can come from several sources; for example soft jets that did not make the $8 \mathrm{GeV}$ cut of Section 3.6 .2 and jet energy outside of the $R=0.5$ cone are both treated as unclustered energy. Only calorimeter cells that went $100 \mathrm{MeV}$ over pedestal are used, in order to reduce the impact from noise in cells, underlying event(s) and pile-up. 
Also, the energy of a tau is corrected for the neutrino(s) it decays into. That energy is missed and would otherwise contribute to our estimate of the primaryneutrino energy [74].

Now, the missing energy is given by

$$
\overrightarrow{\mathbb{E}}_{\mathrm{T}}=\mathbb{E}_{x} \hat{x}+\mathbb{E}_{y} \hat{y} \quad \text { with } \quad \mathbb{E}_{i=x, y}=-\sum_{\text {all objects } j} \vec{p}_{j} \cdot \hat{i},
$$

where $\hat{x}$ and $\hat{y}$ are the unit vectors in the $x$ and $y$ direction.

The uncertainties on object energies are taken from the object resolution (including systematic errors) and the $U_{\mathrm{T}}$ uncertainty is parametrized using the number of jets in the event and the total unclustered energy [75. The uncertainties are projected onto the missing transverse-energy vector and are added orthogonally to form the total $\mathbb{E}_{\mathrm{T}}$ uncertainty. Only the transverse-unclusteredenergy uncertainty, $\sigma_{U_{\mathrm{T}}}$, is added without projection onto the $\mathbb{E}_{\mathrm{T}}$ vector. So, the total variation in missing transverse energy is defined by

$$
\sigma_{\mathscr{E}_{\mathrm{T}}}^{2} \equiv \sigma_{U_{\mathrm{T}}}^{2}+\sum_{\text {all objects } j} \sigma_{j}^{2}\left|\hat{j} \cdot \hat{\mathscr{E}}_{\mathrm{T}}\right| .
$$

Typical values for $\sigma_{\mathscr{E}_{\mathrm{T}}}$ are in the order of $10 \mathrm{GeV}$.

Next, the missing-transverse-energy significance is defined as the log of the ratio of the probability that the $\mathbb{E}_{\mathrm{T}}$ was equal to its measured value and that the $\mathbb{E}_{\mathrm{T}}$ was actually zero:

$$
\mathbb{E}_{\mathrm{T}}^{\mathrm{SIG}} \equiv \log \frac{p\left(\mathbb{E}_{\mathrm{T}}=\mathbb{E}_{\mathrm{T}}^{\text {meas. }}\right)}{p\left(\mathbb{E}_{\mathrm{T}}=0\right)} .
$$

Here, a probability distribution shaped as a Gaussian with mean $\mathbb{E}_{\mathrm{T}}^{\text {meas. }}$ and width $\sigma_{\mathbb{E}_{\mathrm{T}}}$ is assumed.

If $p\left(\mathscr{E}_{\mathrm{T}}=0\right)<e^{-4}$, it is set to $e^{-4}$. This effectively creates an overflow peak so all events with high significance end up in the region $5<\mathbb{E}_{\mathrm{T}}^{\mathrm{SIG}}<7$. This can be seen in Figure 3.14 where the $\mathbb{E}_{\mathrm{T}}^{\mathrm{SIG}}$ distribution is plotted of a data sample with one isolated electron and at least one jet. The QCD background is clearly separated from the region with $W+$ jets events.

Sometimes it is useful to look at the $E_{\mathrm{T}}$ and $\mathbb{E}_{\mathrm{T}}$ with only calorimeter objects taken into account. These are called $H_{\mathrm{T}}$ and $H_{\mathrm{T}}$, respectively.

In Monte Carlo all corrections for the different objects are propagated to the missing transverse energy. 


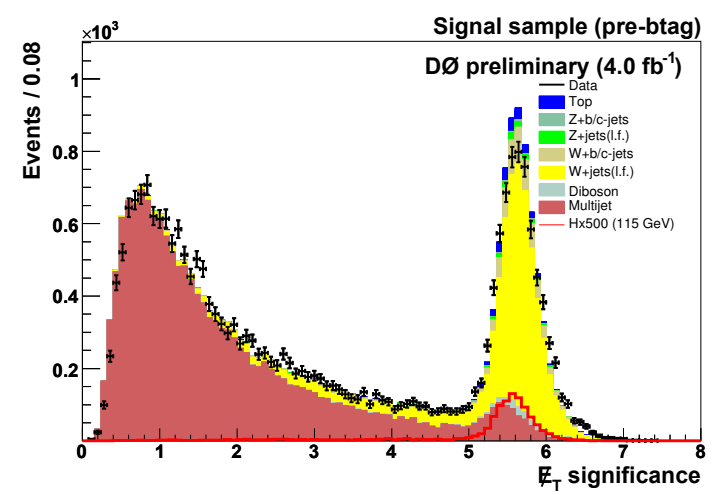

Figure 3.14: histogram of the distribution of missing transverse energy significance compared to multijet and MC-generated backgrounds. For a magnified legend, see Figure $4.3(\mathrm{~d})$. 


\section{Chapter 4}

\section{Event Selection}

This chapter discusses the selection procedure for the $W H \rightarrow \tau \nu b \bar{b}$ analysis. Its descriptions are based on a $D \varnothing$ internal note [76], which in turn is built on the work performed by Philip Rich [77, 78, Differences with previous versions of the analysis are discussed in Section 6.1.

First, data and MC samples are defined to which a pre-selection is applied that cuts the bulk of the background, without sacrificing too many $W H \rightarrow \tau \nu b \bar{b}$ events. In Section 4.3, our background predictions are tested in different control samples. Further selection, discussed in Section 4.4.1 purifies the sample where we expect a possible signal. Finally, events that pass our $b$-tagging requirements are split by $\tau$-type, jet multiplicity and $b$-tag operating point. The result is a set of 8 orthogonal samples that are highly enriched in $W H \rightarrow \tau \nu b \bar{b}$ events. In these samples, the SM Higgs hypotheses will be tested, which is described in Chapter5.

In the following sections, different variables are used to place selection cuts on. Distributions of those variables are shown to verify the consistency between measured data and background predictions. The variables are explained in Table 4.1 as a reference.

\subsection{Data and MC samples}

As a starting point, the $W H \rightarrow \tau \nu b \bar{b}$ analysis uses a pre-defined missing-energy dataset that is used for several analyses in the $\mathrm{D} \varnothing$ collaboration. This is a subset of the entire recorded dataset where events are selected for their likelihood of having a significant amount of missing energy. The triggers used for this skim can be found in Section 4.1.1.

The MC-generated events are passed through a trigger simulation to get optimal MC-data agreement. This is discussed in Section 4.1 .2 
After triggering, 'data quality' cuts are applied and duplicate events are removed. Data-quality cuts include the removal of data-taking periods where one or more important subdetectors were not performing optimally, or not-understood patterns occurred in the data. Table 4.2 lists the luminosity for the trigger periods that are used.

\begin{tabular}{|c|c|}
\hline Variable & Description \\
\hline $\begin{array}{l}\# \text { jets } \\
\text { jet1 }\end{array}$ & $\begin{array}{l}\text { number of jets in the event; we select either } 2 \text { or } 3 \text {-jet events } \\
\text { the highest- } p_{\mathrm{T}} \text { jet }\end{array}$ \\
\hline jet2 & the jet with the next-to-highest $p_{\mathrm{T}}$ \\
\hline$p_{\mathrm{T}}$ & transverse momentum, as described in Section 2.2.1 \\
\hline$\eta$ & pseudorapidity, as described in Section 2.2.1 \\
\hline$\eta_{\mathrm{d}}$ & $\begin{array}{l}\text { detector } \eta \text {; the } \eta \text { in the (sub)detector with respect to the center } \\
\text { of the } D \varnothing \text { detector }\end{array}$ \\
\hline$\Delta R$ & $\begin{array}{l}\text { distance in } \eta \times \phi \text { space between two objects, which is defined } \\
\text { by } \Delta R \equiv \sqrt{\Delta \phi^{2}+\Delta \eta^{2}}\end{array}$ \\
\hline$\Delta R(\tau$, jets $)$ & $\Delta R$ between the $\tau$ candidate and closest jet \\
\hline$m_{\mathrm{T}}(W)$ & $\begin{array}{l}\text { transverse mass of the } \tau \text { and } \mathbb{E}_{\mathrm{T}} \text { combined system, given by } \\
m_{\mathrm{T}}(W)=\sqrt{2 \mathbb{E}_{\mathrm{T}} E_{\mathrm{T}}(\tau)\left(1-\cos \Delta \phi\left(\mathscr{E}_{\mathrm{T}}, \tau\right)\right)}\end{array}$ \\
\hline$m_{\mathrm{jj}}$ & $\begin{array}{l}\text { dijet invariant mass, the combined mass of the two highest- } \\
\text { energetic jets; a variable that shows important differences be- } \\
\text { tween the hypotheses with or without a SM Higgs boson }\end{array}$ \\
\hline $\begin{array}{l}\text { scalar } E_{\mathrm{T}} \\
\mathbb{E}_{\mathrm{T}}\end{array}$ & $\begin{array}{l}\text { scalar sum of all transverse energy in the event } \\
\text { missing transverse energy, as described in Section } 3.9\end{array}$ \\
\hline $\mathbb{E}_{\mathrm{T}}^{\mathrm{SIG}}$ & $\mathbb{E}_{\mathrm{T}}$ significance, as described in Section 3.9 \\
\hline$\not p_{\mathrm{T}}^{\mathrm{trk}}$ & $\begin{array}{l}\text { missing } p_{\mathrm{T}} \text { when only counting the transverse momentum of } \\
\text { tracks }\end{array}$ \\
\hline$\Delta \phi\left(\not p_{\mathrm{T}}^{\mathrm{trk}}, \mathbb{E}_{\mathrm{T}}\right)$ & $\begin{array}{l}\text { angle in } \phi \text { between the } \not p_{\mathrm{T}} \text { from tracks and the } \mathbb{E}_{\mathrm{T}} \text {; a useful } \\
\text { variable to identify instrumental background }\end{array}$ \\
\hline$H_{\mathrm{T}}$ & scalar $E_{\mathrm{T}}$ if only jets are taken into account \\
\hline$H_{\mathrm{T}}($ jets,$\tau)$ & scalar $E_{\mathrm{T}}$ if only jets and the tau are taken into account \\
\hline$\not H_{\mathrm{T}}$ & $\mathscr{E}_{\mathrm{T}}$ if only jets are taken into account (Section 3.9 ) \\
\hline A & $\begin{array}{l}\text { 'asymmetry' between } \mathbb{E}_{\mathrm{T}} \text { and } \mathbb{H}_{\mathrm{T}}: A \equiv\left(\mathbb{E}_{\mathrm{T}}-\mathbb{H}_{\mathrm{T}}\right) /\left(\mathbb{E}_{\mathrm{T}}+H_{\mathrm{T}}\right), \\
\text { which is a useful variable to identify instrumental background }\end{array}$ \\
\hline
\end{tabular}

Table 4.1: descriptions of variables important to the $W H \rightarrow \tau \nu b \bar{b}$ analysis. 


\begin{tabular}{llrrr}
\hline Trigger list & Trigger & Delivered & Recorded & Good \\
\hline v12.10 - v12.40 & MHT30_3CJT5 & 277.1 & 250.0 & 227.5 \\
v13.00 - v14.00 & JT1_ACO_MHT_HT & 464.0 & 425.5 & 378.6 \\
v14.00 - v15.00 & JT1_ACO_MHT_HT & 415.2 & 394.0 & 339.2 \\
\hline Total Run IIa & & 1156.3 & 1069.5 & 945.2 \\
v15.00 - v15.20 & JT1_ACO_MHT_HT & 321.1 & 250.5 & 209.5 \\
v15.20 - v16.00 & JT1_MHTACO & 1615.3 & 1535.3 & 1415.6 \\
v16.00 - v16.32 & JT1_MHTACO & 1616.6 & 1552.0 & 1407.3 \\
\hline Total Run IIb & & 3553.0 & 3337.8 & 3032.4 \\
\hline
\end{tabular}

Table 4.2: Run IIa and Run IIb triggers with luminosity in $\mathrm{pb}^{-1}$ delivered by the Tevatron, recorded by D $\varnothing$ and with 'good' data quality.

\subsubsection{Triggering on Data}

A full $\mathrm{D} \varnothing$ trigger overview is listed in Section 2.3. For the Run IIa period of the $W H \rightarrow \tau \nu b \bar{b}$ analysis, two calorimeter-based triggers were used. Trigger version 12 used 'MHT30_3CJT5' as trigger. A trigger called 'JT1_ACO_MHT_HT' was used in versions 13 and 14 . These trigger versions have the following implementation for the three trigger levels:

Level 1 uses triggers called $\operatorname{CJT}(X, Y, Z)$. They are based on the amount of calorimeter trigger towers $(X)$, which have at least a certain transverse energy $(Y \mathrm{GeV})$ and are within an $\eta_{\mathrm{d}}$ range $\left(\left|\eta_{\mathrm{d}}\right|<Z\right)$. Versions 12 and 13 use only $\operatorname{CJT}(3,5,3.2)$. In version $14 \operatorname{CJT}(3,5,3.2)$ OR $\operatorname{CJT}(3,4,2.4)$ is required.

Level 2 demands $\not H_{\mathrm{T}}>20 \mathrm{GeV}$, where only jets with $E_{\mathrm{T}}>10 \mathrm{GeV}$ are taken into account. In versions 13 and 14, also the $\phi$ angle between the two highest-energy jets, $\Delta \phi\left(\right.$ jet1,jet2), needs to be below $168.75^{\circ}$.

Level 3 has an improved calculation of many variables (see Section 2.3.4). It requires $H_{\mathrm{T}}>30 \mathrm{GeV}$. Furthermore $H_{\mathrm{T}}>50 \mathrm{GeV}$ is demanded for versions 13 and 14. The $\phi$ angle between the two highest-energy jets needs to be below $170^{\circ}$. In version 14 an additional requirement is that the $\phi$ angle between the direction of the $H_{\mathrm{T}}$ and the closest jet should be at least $25^{\circ}$. At level 3 only jets with $E_{\mathrm{T}}>9 \mathrm{GeV}$ are taken into account.

In Table 4.3 the implementation in terms of cuts on these variables is shown for the trigger schemes of Run IIa. 


\begin{tabular}{|c|c|c|c|c|}
\hline & & \multirow{2}{*}{$\begin{array}{c}\text { MHT30_3CJT5 } \\
\text { v12 }\end{array}$} & \multicolumn{2}{|c|}{ JT1_ACO_MHT_HT } \\
\hline & & & v13 & v14 \\
\hline \multirow{2}{*}{ L1 } & $\operatorname{CJT}(3,5,3.2)$ & $\mathrm{X}$ & $\mathrm{X}$ & $\mathrm{X}$ \\
\hline & $\operatorname{CJT}(3,4,2.4)$ & & & $\mathrm{X}$ \\
\hline \multirow{2}{*}{$\mathrm{L} 2$} & $\not H_{\mathrm{T}}>20, E_{\mathrm{T}}^{\mathrm{jet}}>10$ & $\mathrm{X}$ & $\mathrm{X}$ & $\mathrm{X}$ \\
\hline & $\Delta \phi($ jet1,jet2 $)<168.75^{\circ}$ & & $\mathrm{X}$ & $\mathrm{X}$ \\
\hline \multirow{4}{*}{ L3 } & $H_{\mathrm{T}}>30, E_{\mathrm{T}}^{\mathrm{jet}}>9$ & $\mathrm{X}$ & $\mathrm{X}$ & $\mathrm{X}$ \\
\hline & $H_{\mathrm{T}}>50, E_{\mathrm{T}}^{\mathrm{jet}}>9$ & & $\mathrm{X}$ & $\mathrm{X}$ \\
\hline & $\Delta \phi($ jet 1, jet 2$)<170^{\circ}$ & & $\mathrm{X}$ & $\mathrm{X}$ \\
\hline & $\Delta \phi\left(\right.$ jets, $\left.\not H_{\mathrm{T}}\right)>25^{\circ}$ & & & $\mathrm{X}$ \\
\hline
\end{tabular}

Table 4.3: summary of the trigger scheme in Run IIa trigger versions. An ' $\mathrm{X}$ ' denotes the trigger is used.

For Run IIb, two calorimeter-based triggers were used, like for Run IIa. During version $15 \mathrm{JT} 1 \_\mathrm{ACO} \_\mathrm{MHT} \_\mathrm{HT}$ was still used. Versions 15.2 and 16 were two realizations of $\overline{\mathrm{JT}} 1 \_\overline{\mathrm{MHTACO}}$.

Level 1 uses in all Run IIb versions:

- $\mathbb{E}_{\mathrm{T}}>24 \mathrm{GeV}$ AND CJT(1,30,3.2); OR

- $\mathbb{E}_{\mathrm{T}}>24 \mathrm{GeV}$ AND CJT $(1,20,2.4)$ AND CJT(2,8,2.4) AND ACOKILL; OR

- $\operatorname{CJT}(1,30,2.4)$ AND CJT(2, 15, 2.4) AND CJT(3, 8, 3.2),

where ACOKILL requires all pairs of jets with $8 \mathrm{GeV}<E_{\mathrm{T}}^{\text {jet }}<20 \mathrm{GeV}$ to not be back-to-back; the pairs need to have $\Delta \phi<175^{\circ}$.

Level 2 obliges events to pass:

- $\operatorname{CJT}(1,20,2.4) ; \mathrm{AND}$

- $H_{\mathrm{T}}>20 \mathrm{GeV}$ for $E_{\mathrm{T}}^{\text {jet }}>10 \mathrm{GeV}$ jets; AND

- $H_{\mathrm{T}}>35 \mathrm{GeV}$ for jets with $E_{\mathrm{T}}^{\text {jet }}>6 \mathrm{GeV}$ and $\left|\eta_{\mathrm{d}}^{\text {jet }}\right|<2.6$; AND

- $\Delta \phi\left(\right.$ jets, $\left.\not H_{\mathrm{T}}\right)>25^{\circ}$.

Level 3 requires:

- $\Delta \phi($ jet1,jet 2$)<170^{\circ} ; \mathrm{AND}$

- $\Delta \phi\left(\right.$ jets, $\left.\not H_{\mathrm{T}}\right)>25^{\circ}$. 
- In version 15 , there also has to be at least one jet with $E_{\mathrm{T}}>9 \mathrm{GeV}$, $H_{\mathrm{T}}$ should be greater than $50 \mathrm{GeV}$, and $H_{\mathrm{T}}>30 \mathrm{GeV}$.

- For versions 15.2 and 16 , at least two jets with $E_{\mathrm{T}}>9 \mathrm{GeV}$ are required, along with $\not_{\mathrm{T}}>25 \mathrm{GeV}$ and $\mathbb{E}_{\mathrm{T}}>25 \mathrm{GeV}$.

\subsubsection{Monte Carlo Datasets}

Using the event generators discussed in Section 2.4 different MC samples were generated for both data taking periods, to account for the changes in detector geometry. The physics modes, cross sections and number of generated events for these samples are shown in Table 4.4 for signal MC and in Tables 4.5 and 4.6 for background events. The number of events after applying data-quality cuts and duplicate-event removal is mentioned. Samples are generated in bins of the number of light partons (lp).

The number of expected events of a process is given by the cross section of that process $\sigma$, times the integrated luminosity $L$. Therefore, the generated MC events of the process get a weight $w$, calculated as

$$
w=\sigma L / N
$$

where $N$ is the number of generated events for the process.

\begin{tabular}{clrrr}
\hline & \multicolumn{2}{c}{$Z H \rightarrow l \bar{l} b \bar{b}$} & \multicolumn{2}{c}{$W H \rightarrow \ell \nu b \bar{b}$} \\
$m_{H}(\mathrm{GeV})$ & $\sigma(\mathrm{pb})$ & $\#$ of events & \multicolumn{1}{c}{$\sigma(\mathrm{pb})$} & $\#$ of events \\
\hline 100 & 0.01368 & 521974 & 0.07528 & 516709 \\
105 & 0.01149 & 490779 & 0.06271 & 488855 \\
110 & 0.00959 & 523075 & 0.05183 & 516682 \\
115 & 0.00789 & 481579 & 0.04225 & 448993 \\
120 & 0.00635 & 534451 & 0.03368 & 518018 \\
125 & 0.00496 & 474283 & 0.02611 & 474079 \\
130 & 0.00374 & 516854 & 0.01956 & 749582 \\
135 & 0.00271 & 474432 & 0.01404 & 666576 \\
140 & 0.00188 & 514145 & 0.00964 & 695630 \\
145 & 0.00123 & 473694 & 0.00626 & 644528 \\
150 & 0.00074 & 512211 & 0.00377 & 516272 \\
\hline
\end{tabular}

Table 4.4: cross section and number of generated events for each Higgs boson mass and signal MC sample. The $Z$ can decay into any lepton $l$, including neutrinos, while $\ell$ stands for charged leptons only. 


\begin{tabular}{|c|c|c|c|}
\hline \multirow[b]{2}{*}{ Mode } & \multirow[b]{2}{*}{$\sigma(\mathrm{pb})$} & \multicolumn{2}{|c|}{ Number of events } \\
\hline & & Run IIa & Run IIb \\
\hline $\mathrm{WZ} \rightarrow$ any & 3.25 & 590647 & 632296 \\
\hline $\mathrm{WW} \rightarrow$ any & 11.6 & 2457974 & 709879 \\
\hline $\mathrm{ZZ} \rightarrow$ any & 1.33 & 590647 & 540273 \\
\hline$W+0 \mathrm{lp}$ & 4510 & 12526514 & 46404567 \\
\hline$W+1 l p$ & 1280 & 9425157 & 19898756 \\
\hline$W+2 \mathrm{lp}$ & 304 & 4285002 & 18087996 \\
\hline$W+3 \operatorname{lp}$ & 72.6 & 3535816 & 3754272 \\
\hline$W+4 \operatorname{lp}$ & 16.8 & 2493506 & 2602738 \\
\hline$W+\geq 5 \mathrm{lp}$ & 5.15 & 780938 & 2044335 \\
\hline$W+c c+0 \operatorname{lp}$ & 24.5 & 1205996 & 934253 \\
\hline$W+c c+1 \operatorname{lp}$ & 13.5 & 740093 & 738709 \\
\hline$W+c c+2 \operatorname{lp}$ & 5.5 & 342472 & 554236 \\
\hline$W+c c+\geq 3 \mathrm{lp}$ & 2.53 & 446288 & 469900 \\
\hline$W+b b+0 \operatorname{lp}$ & 9.37 & 1372108 & 1104413 \\
\hline$W+b b+1 l p$ & 4.3 & 666553 & 782487 \\
\hline$W+b b+2 \mathrm{lp}$ & 1.57 & 248702 & 523717 \\
\hline$W+b b+\geq 3 \mathrm{lp}$ & 0.724 & 276900 & 412747 \\
\hline$(t \rightarrow e+\nu+b)+b+0 \mathrm{lp}$ & 0.112 & 290262 & 247517 \\
\hline$(t \rightarrow \mu+\nu+b)+b+0 \mathrm{lp}$ & 0.11 & 287994 & 225286 \\
\hline$(t \rightarrow \tau+\nu+b)+b+0 \mathrm{lp}$ & 0.117 & 287991 & 248722 \\
\hline$(t \rightarrow e+\nu+b)+b+\geq 1 \mathrm{lp}$ & 0.243 & 289325 & 272573 \\
\hline$(t \rightarrow \mu+\nu+b)+b+\geq 1 \mathrm{lp}$ & 0.239 & 288444 & 273354 \\
\hline$(t \rightarrow \tau+\nu+b)+b+\geq 1 \mathrm{lp}$ & 0.254 & 289106 & 246552 \\
\hline$(t \bar{t} \rightarrow 2 b+4 \mathrm{lp})+0 \mathrm{lp}$ & 1.42 & 771121 & 793267 \\
\hline$(t \bar{t} \rightarrow 2 b+4 \mathrm{lp})+1 \mathrm{lp}$ & 0.576 & 487256 & 456317 \\
\hline$(t \bar{t} \rightarrow 2 b+4 \mathrm{lp})+\geq 2 \mathrm{lp}$ & 0.281 & 292017 & 277912 \\
\hline$(t \bar{t} \rightarrow \ell+\nu+2 b+2 \mathrm{lp})+0 \mathrm{lp}$ & 1.4 & 771271 & 777068 \\
\hline$(t \bar{t} \rightarrow \ell+\nu+2 b+2 \mathrm{lp})+1 \mathrm{lp}$ & 0.577 & 492647 & 457782 \\
\hline$(t \bar{t} \rightarrow \ell+\nu+2 b+2 \mathrm{lp})+\geq 2 \mathrm{lp}$ & 0.267 & 288992 & 321166 \\
\hline$(t \bar{t} \rightarrow 2 \ell+2 \nu+2 b)+0 \mathrm{lp}$ & 0.352 & 1516107 & 749642 \\
\hline$(t \bar{t} \rightarrow 2 \ell+2 \nu+2 b)+1 \mathrm{lp}$ & 0.142 & 963057 & 452177 \\
\hline$(t \bar{t} \rightarrow 2 \ell+2 \nu+2 b)+\geq 2 \mathrm{lp}$ & 0.0678 & 701167 & 281453 \\
\hline
\end{tabular}

Table 4.5: cross sections and number of generated events of diboson, $W+$ jets, single top and $t \bar{t}$ MC samples. The decay in diboson events is unrestricted, but only leptonic decays of the $W$ are considered in $W+$ jets events. In $t \bar{t}$ decays, $\ell$ stands for any charged lepton. 


\begin{tabular}{|c|c|c|c|}
\hline \multirow[b]{2}{*}{ Mode } & \multirow[b]{2}{*}{$\sigma(\mathrm{pb})$} & \multicolumn{2}{|c|}{ Number of events } \\
\hline & & Run IIa & Run IIb \\
\hline$(Z \rightarrow \mu \mu)+0 \operatorname{lp}$ & 479 & 3900665 & 3591594 \\
\hline$(Z \rightarrow \mu \mu)+1 \mathrm{lp}$ & 81.9 & 2717367 & 1345143 \\
\hline$(Z \rightarrow \mu \mu)+2 \mathrm{lp}$ & 19.88 & 1384893 & 835902 \\
\hline$(Z \rightarrow \mu \mu)+\geq 3 \operatorname{lp}$ & 6.12 & 1262074 & 556145 \\
\hline$(Z \rightarrow \tau \tau)+0 \operatorname{lp}$ & 470 & 3664007 & 3407959 \\
\hline$(Z \rightarrow \tau \tau)+1 \mathrm{lp}$ & 80.6 & 2492966 & 1262046 \\
\hline$(Z \rightarrow \tau \tau)+2 \mathrm{lp}$ & 20.09 & 1114447 & 716208 \\
\hline$(Z \rightarrow \tau \tau)+\geq 3 \mathrm{lp}$ & 5.91 & 989593 & 609694 \\
\hline$(Z \rightarrow \nu \nu)+\overline{0 l p}$ & 805 & 1921328 & 2308113 \\
\hline$(Z \rightarrow \nu \nu)+1 \mathrm{lp}$ & 246 & 1856981 & 2449272 \\
\hline$(Z \rightarrow \nu \nu)+2 \operatorname{lp}$ & 61 & 883662 & 590022 \\
\hline$(Z \rightarrow \nu \nu)+3 \mathrm{lp}$ & 14.1 & 248300 & 166856 \\
\hline$(Z \rightarrow \nu \nu)+4 \mathrm{lp}$ & 3.28 & 81974 & 82479 \\
\hline$(Z \rightarrow \nu \nu)+\geq 5 \mathrm{lp}$ & 0.936 & 38273 & 38872 \\
\hline$(Z \rightarrow \mu \mu)+c c+0 \operatorname{lp}$ & 5.08 & 486805 & 454001 \\
\hline$(Z \rightarrow \mu \mu)+c c+1 l p$ & 1.51 & 250059 & 231694 \\
\hline$(Z \rightarrow \mu \mu)+c c+\geq 2 \mathrm{lp}$ & 0.627 & 196752 & 194659 \\
\hline$(Z \rightarrow \tau \tau)+c c+0 \mathrm{lp}$ & 5.02 & 494301 & 532224 \\
\hline$(Z \rightarrow \tau \tau)+c c+1 \mathrm{lp}$ & 1.54 & 240629 & 331018 \\
\hline$(Z \rightarrow \tau \tau)+c c+\geq 2 \operatorname{lp}$ & 0.683 & 194688 & 276512 \\
\hline$(Z \rightarrow \nu \nu)+c c+\overline{0} \operatorname{lp}$ & 5.61 & 202417 & 376456 \\
\hline$(Z \rightarrow \nu \nu)+c c+1 \mathrm{lp}$ & 3 & 96503 & 199012 \\
\hline$(Z \rightarrow \nu \nu)+c c+\geq 2 \operatorname{lp}$ & 1.64 & 50589 & 96147 \\
\hline$(Z \rightarrow \mu \mu)+b b+0 \mathrm{lp}$ & 0.936 & 550215 & 477319 \\
\hline$(Z \rightarrow \mu \mu)+b b+1 l p$ & 0.396 & 236209 & 226102 \\
\hline$(Z \rightarrow \mu \mu)+b b+\geq 2 \mathrm{lp}$ & 0.1783 & 179404 & 166163 \\
\hline$(Z \rightarrow \tau \tau)+b b+0 l p$ & 0.937 & 495651 & 463345 \\
\hline$(Z \rightarrow \tau \tau)+b b+1 \mathrm{lp}$ & 0.388 & 232025 & 232019 \\
\hline$(Z \rightarrow \tau \tau)+b b+\geq 2 \mathrm{lp}$ & 0.1851 & 181708 & 165623 \\
\hline$(Z \rightarrow \nu \nu)+b b+0 \mathrm{lp}$ & 2.52 & 388118 & 367482 \\
\hline$(Z \rightarrow \nu \nu)+b b+11 \mathrm{p}$ & 1.14 & 190191 & 171942 \\
\hline$(Z \rightarrow \nu \nu)+b b+\geq 2 \mathrm{lp}$ & 0.617 & 88683 & 82829 \\
\hline
\end{tabular}

Table 4.6: cross section and number of generated events of $Z+$ jets MC samples. 


\section{Trigger Parameterization}

A simulated trigger is applied to the MC to mimic the detector's trigger response. This analysis makes use of the parameterizations resulting from the trigger simulation used by the $Z H \rightarrow \nu \nu b \bar{b}$ analysis [79 81 . The associated systematic uncertainties are estimated by applying the trigger-decision parametrization to muon-triggered $W \rightarrow \mu \nu$ events. The resulting $m_{\mathrm{jj}}$ distribution is compared with the distribution of $W \rightarrow \mu \nu$ events that are directly triggered by our calorimeter based triggers.

Additional care is taken that the tau candidate is treated as a jet both in data and in MC. This is especially important in trigger level 3, where topological variables like $H_{\mathrm{T}}$ and $H_{\mathrm{T}}$ would not be well described otherwise. If not taken into account, this would lead to inefficiencies in the $\mathrm{MC}$ that are not present in data.

To avoid double counting in the trigger parameterization, jets and electrons that overlap taus are removed from the corresponding jet and electron lists that are passed to the trigger parameterization. This correctly reflects the way these objects are modeled in the data trigger, where objects overlapping taus are considered as taus only.

In Section 5.2.1 the systematic uncertainties related to the trigger parameterization will be discussed.

\section{Reweighting of Boson Transverse Momentum}

The analysis uses the standard $\mathrm{D} \varnothing$ corrections of $p_{\mathrm{T}}(Z)$ and $p_{\mathrm{T}}(W)$ reweighting. These reweightings address the mismodeling of the $p_{\mathrm{T}}$ distributions of the $Z$ and $W$ bosons in Monte Carlo, compared to those measured in data. Events with $Z \rightarrow e e$ are used to determine the reweighting in bins of the $p_{\mathrm{T}}(Z)$ [82, 83]. In Figure 4.1 the ratio of data over MC on which the reweighting is based is shown.

For $p_{\mathrm{T}}(W)$ reweighting another method has to be used, because leptonic decays of the $W$ have a neutrino, making a $p_{\mathrm{T}}$ measurement very hard. Therefore, the ratio of the number of $W$ to the number of $Z$ events is used, which is known at NLO, but is not well reproduced by MC simulation [84. Therefore data events from leptonic $Z$ decays are multiplied with the $W / Z$ ratio to get a 'measured' number of $W \mathrm{~s}$, on which the reweighting can be determined. In Figure 4.2 the ratio of $(Z$ data $) \times(\mathrm{NLO} W / Z)$ over MC-generated $W$ events can be seen.

\subsection{Preselection}

After choosing the initial data and Monte-Carlo samples, a so-called preselection is run. This rejects events that hardly contribute to finding $W H \rightarrow \tau \nu b \bar{b}$. The 


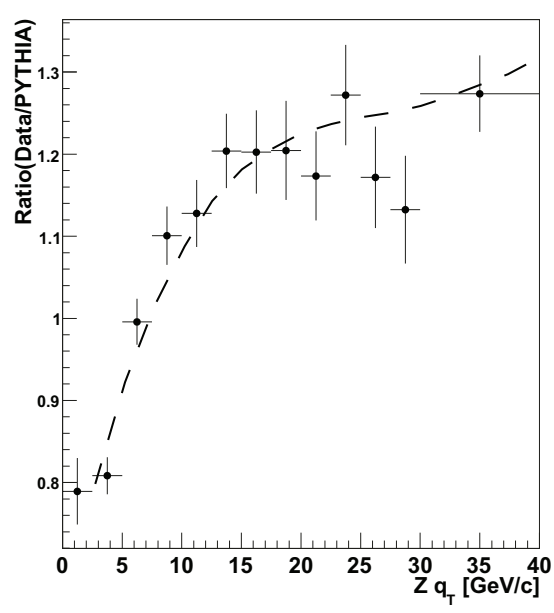

(a) ratio of data to Pythia $\mathrm{MC}$

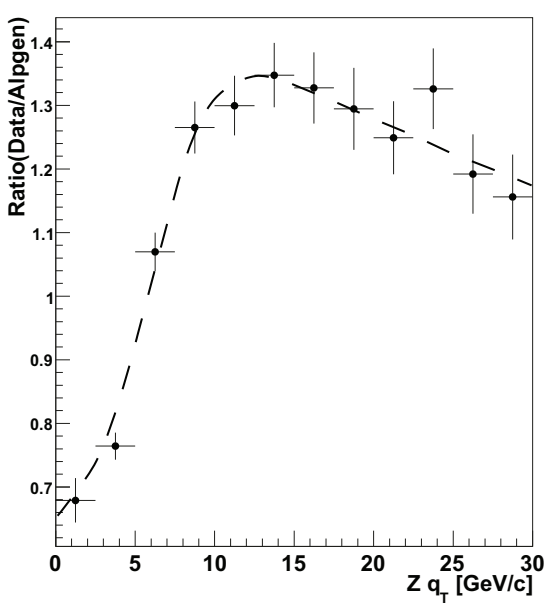

(b) ratio of data to ALPGEN MC

Figure 3: Ratio of $Z q_{T}$ in unfolded faitgaure 13: Ratio of $Z q_{T}$ in unfolded data

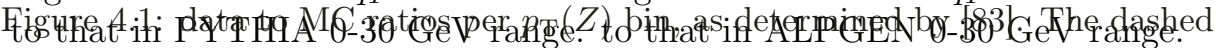
lines indicate the applied corrections.

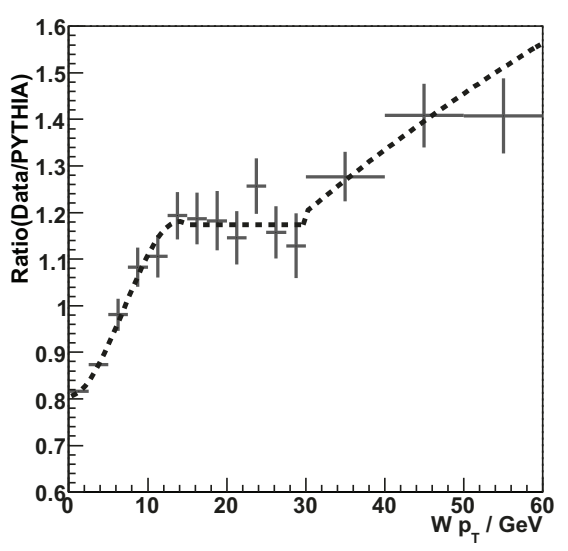

(a) ratio of data to Pythia $\mathrm{MC}$

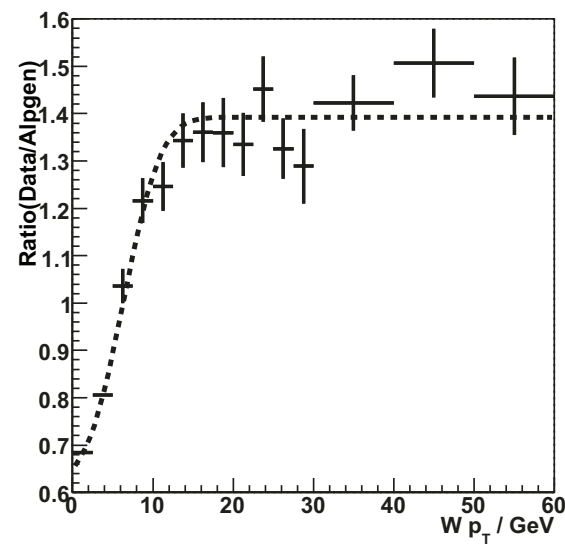

(b) ratio of data to ALPGEN MC

Figure 4.2: data to $\mathrm{MC}$ ratios per $p_{\mathrm{T}}(W)$ bin, where 'data' is determined by a $Z$ data sample times the NLO ratio of $W / Z$ [84]. The $p_{\mathrm{T}}(W)$ corrections are shown here as dotted lines. 
total number of events is reduced by a substantial factor, making subsequent analyses much faster. In this section, it will be discussed how cuts are placed on properties of taus, jets, electrons and muons. Descriptions of these objects can be found in Chapter 3 Also, a description is given of how events used in complementary analyses are vetoed ascertaining that the different analyses are statistically independent.

\subsubsection{Tau Selection}

Taus are identified, as is explained in Section 3.8, using the hadronic tau Neural Network $\mathrm{NN}_{\tau}$.

At least one tau candidate passing tau-type dependent cuts is required in the event. For both tau types, $\mathrm{NN}_{\tau}>0.3$ is required at preselection level. Also, the tau candidate should have $\left|\eta_{\mathrm{d}}\right|<2$.

Cuts on the momentum of the track associated to the tau, $p_{\mathrm{T}}^{\operatorname{trk}}(\tau)$, and the momentum measured by the calorimeter, $p_{\mathrm{T}}(\tau)$, depend on the tau type. We use only tau types 1 and 2 for this analysis, because for tau type 3 the multijet background is too high. For type 1 taus we require $p_{\mathrm{T}}(\tau)>12 \mathrm{GeV}$, and $p_{\mathrm{T}}^{\text {trk }}(\tau)>$ $7 \mathrm{GeV}$, while for type 2 taus the cuts are $p_{\mathrm{T}}>10 \mathrm{GeV}$ and $p_{\mathrm{T}}^{\text {trk }}(\tau)>5 \mathrm{GeV}$.

\subsubsection{Jet Selection}

We require 2 or 3 jets with $p_{\mathrm{T}}>20 \mathrm{GeV}$ and $\left|\eta_{\mathrm{d}}\right|<2.5$ in the event. These jets must be separated from the tau candidate by $\Delta R(\tau$, jet $)>0.5$. In Run IIa the two leading jets also need to be in the $\mathrm{CC}$ or EC region, so with $\left|\eta_{\mathrm{d}}\right|<1.1$ or $1.4<\left|\eta_{\mathrm{d}}\right|<2.5$.

We additionally require two jets to be taggable (see Section 3.7). For Run IIb, with a significant number of interactions per crossing possible, we also demand the taggable jets to be vertex confirmed, meaning that they have at least two tracks associated with the primary vertex. These requirements remove a large fraction of the instrumental background by rejecting events with fake jets, or events where the wrong primary vertex was picked.

Reconstructed jets are corrected based on the expected calorimeter response, energy loss due to showering out of the jet cone, and energy deposited in the jet cone not associated with the jet, as explained in Section 3.6 .

\subsubsection{Electron and Muon Removal}

Any events selected by the $W H \rightarrow e \nu b \bar{b}$ and $W H \rightarrow \mu \nu b \bar{b}$ analyses [85, 86] are removed from our sample. The analysis also has to remain orthogonal to the $H+X \rightarrow \ell^{ \pm} \tau^{\mp} j j[87,88]$ analysis. As hardly any signal acceptance is gained in this analysis by retaining muons, a simple veto on events with isolated muons is 
employed. An event is vetoed if it has a muon with quality criteria 'loose' with hits in the A plus $\mathrm{B}$ or $\mathrm{C}$ segments, $p_{\mathrm{T}}^{\mu}>15 \mathrm{GeV}$ and no cosmic veto. It must also be matched to a central track. Section 3.4 contains more information on muon identification. In addition, the following cuts are required:

- The distance at closest approach, $\left|d_{0}\right|$, of tracks with SMT hits $<0.02$;

- $\left|d_{0}\right|$ of tracks without SMT hits $<0.2$;

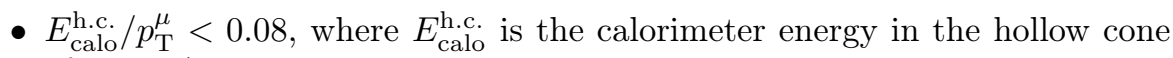
of $0.1<\Delta R<0.4$ around the muon;

- $p_{\mathrm{T}}^{\text {tracks }} / p_{\mathrm{T}}^{\mu}<0.06$, where $p_{\mathrm{T}}^{\text {tracks }}$ is the scalar sum of the $p_{\mathrm{T}}$ in all tracks which have a $\Delta R$ with the muon smaller than 0.5 , except the muon track itself;

- $\chi^{2} /$ d.o.f. from global matching $<4$; and

- $\Delta R$ from jets $>0.5$.

For orthogonality to the $W H \rightarrow e \nu b \bar{b}$ channel, other topological cuts are also considered to prevent loss of signal acceptance; we only remove the events with isolated electrons from our analysis if the events also passed the other $W H \rightarrow$ $e \nu b \bar{b}$ analysis cuts. This is done in order not to lose electrons that fake tau candidates but are not included in other analyses. An electron event is vetoed if it has a $p_{\mathrm{T}}>15 \mathrm{GeV}$ electron either in the $\mathrm{CC}$ or EC region of the detector. See Section 3.5 for electron identification at $\mathrm{D} \varnothing$. The event must also pass the following cuts to be vetoed:

- $\mathbb{E}_{\mathrm{T}}>20 \mathrm{GeV}$ if $\left|\eta_{\mathrm{d}}\right|<1.1$;

- $\mathbb{E}_{\mathrm{T}}>25 \mathrm{GeV}$ if $1.5<\left|\eta_{\mathrm{d}}\right|<2.5$;

- $m_{\mathrm{T}}(W)>\left(40-0.5 \mathbb{E}_{\mathrm{T}}\right)$;

- $p_{\mathrm{T}}($ jet1) $>25 \mathrm{GeV}$;

- two or more vertex confirmed jets with $p_{\mathrm{T}}>20 \mathrm{GeV}$ and $\left|\eta_{\mathrm{d}}\right|<2.5$; and

- $H_{\mathrm{T}}<60 \mathrm{GeV}$.

\subsubsection{Vertex Requirements}

The primary vertex (PV) should have at least 3 tracks associated with it, and its $z$ position, $\left|z_{\mathrm{PV}}\right|$, should be within $60 \mathrm{~cm}$ from the center of the detector, which is consistent with the beam profile. Tracks and vertices are described in Section 3.1 and Section 3.3 , respectively.

The number of events passing the preselection cuts on the data and signal MC samples for the full Run II dataset is shown in Table 4.7. 


\begin{tabular}{lrrr}
\hline Preselection cut & Recorded data & $W H \rightarrow \ell \nu b \bar{b}$ & $Z H \rightarrow \ell \bar{\ell} b \bar{b}$ \\
\hline Initial dataset & $140,254,517$ & 447,284 & 479,987 \\
Data quality & $119,904,816$ & 446,403 & 479,085 \\
Event quality & $108,175,790$ & 432,412 & 464,282 \\
Tau kinematic cuts & $19,663,876$ & 339,630 & 386,382 \\
Tau NN cut & $3,930,981$ & 159,485 & 231,819 \\
$2 \leq N_{\text {jets }} \leq 3$ & 941,017 & 90,992 & 136,206 \\
Trigger Simulation & 941,017 & 32,823 & 18,986 \\
$\mid z_{\text {PV }} \leq 60$ cm & 926,242 & 32,767 & 18,927 \\
PV has $\geq 3$ tracks & 925,818 & 32,753 & 18,924 \\
\hline
\end{tabular}

Table 4.7: the number of data and signal MC events that passed the initial preselection cuts. The quoted signal $\mathrm{MC}$ has $m_{H}=115 \mathrm{GeV}$.

\subsubsection{Multijet Estimation}

Multijet background is very hard to model using Monte Carlo. This is background coming from QCD events that are badly reconstructed, so missing transverse energy and a tau are faked. ${ }^{1}$ Although the probability for a QCD event to fake both missing energy and a tau is very low, the enormous cross section for those events makes it the dominant background after preselection.

The difficulty to model this background using Monte Carlo is that out of the many generated QCD events, only very few pass the trigger simulation. Therefore the number of generated QCD events that is needed to create a statistically relevant sample after preselection is far higher than what is computationally realistic. Also, the QCD events can only fake a tau and missing $E_{\mathrm{T}}$ because of instrumental errors. This behavior requires a perfect or next-to-perfect understanding of the detector including all its imperfections, so even a statistically significant QCD sample would probably have a large mismodeling of this background.

To model the multijet background, a 'sideband region' is chosen in data. It should consist mainly of QCD events and should have no overlap with the region where further analysis will be performed: the signal region. Any MC generated background in the sideband region is subtracted, leaving only QCD events. The number of QCD events in the signal region of a sample, $\mathrm{QCD}^{\text {sample }}$, can then be expressed as

$$
\mathrm{QCD}^{\text {sample }}=C\left(\text { Data }_{\mathrm{sb}}^{\mathrm{sample}}-\mathrm{MC}_{\mathrm{sb}}^{\text {sample }}\right),
$$

\footnotetext{
${ }^{1}$ For the purpose of this analysis, QCD events are all events without an electroweak interaction in the hard scattering, excluding top production. Although top production is dominated by the strong interaction, it is modeled using MC because the top quark often decays with real $\mathbb{E}_{\mathrm{T}}$.
} 


\begin{tabular}{lccc}
\hline Sample & $\mathbb{E}_{\mathrm{T}}^{\mathrm{SIG}}$ & $\Delta \phi\left(\not p_{\mathrm{T}}^{\mathrm{trk}}, \mathbb{E}_{\mathrm{T}}\right)$ & $\mathbb{E}_{\mathrm{T}}(\mathrm{GeV})$ \\
\hline MC control sample & - & - & $>80$ \\
QCD control sample & $<3.5$ & $>2$ & - \\
Signal sample & $>4.5$ & $<2$ & - \\
\hline
\end{tabular}

Table 4.8: cuts after preselection for the QCD control, MC control and signal samples. Distributions of these variables are shown in Figure 4.3 .

where the number of data and MC events in the sideband region of the sample are denoted as Data ${ }_{\mathrm{sb}}^{\text {sample }}$ and $\mathrm{MC}_{\mathrm{sb}}^{\text {sample }}$, respectively. $C$ represents the ratio of the number of QCD events in the signal region over the number of QCD events in the sideband region. $C$ will be determined using a QCD-dominated sample (see Section 4.3.4 and will henceforward be applied to other samples.

We take $\mathrm{NN}_{\tau}>0.9$ to be the signal region and use $0.3<\mathrm{NN}_{\tau}<0.7$ as the sideband region. The upper limit is chosen at 0.7 as for $\mathrm{NN}_{\tau}>0.7$ a significant fraction of real taus would begin to enter the sample 72 and invalidate the assumption that this region is dominated by fake taus.

\subsection{Control Samples}

The multijet and MC-generated backgrounds need to be investigated to check whether they model the measured data correctly. For this purpose, two control samples are selected: a QCD control sample and a MC control sample. These samples will be used to check our multijet and $\mathrm{MC}$ backgrounds and to determine reweighting factors if needed. Additionally, a signal sample is selected where the Higgs boson search is performed. The reweightings determined in the QCD and MC control samples are applied to the signal sample.

In each sample, the signal region is investigated, so the sample's multijet contribution can be estimated by taking events in the sideband region, while keeping all other criteria.

The criteria for the different samples are listed in Table 4.8 . Figure 4.3 shows the variables on which these sample cuts are placed.

\subsubsection{Control Sample}

For the MC control sample, a region with minimal multijet contamination and low signal content is needed. To achieve this, the MC control sample uses events with $\mathbb{E}_{\mathrm{T}}>80 \mathrm{GeV}$. This yields a sample dominated by $W+$ jets events, as can be seen in Figure 4.3(c) This sample is used to determine any overall normalization 


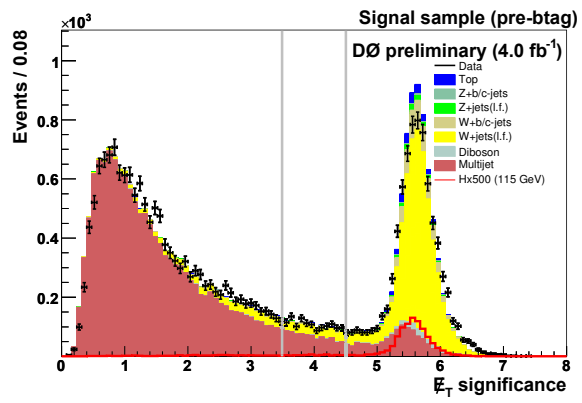

(a) $\mathbb{E}_{\mathrm{T}}^{\mathrm{SIG}}$

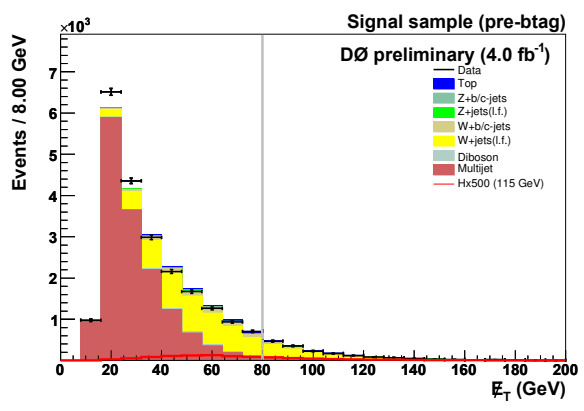

(c) $\mathbb{E}_{\mathrm{T}}$

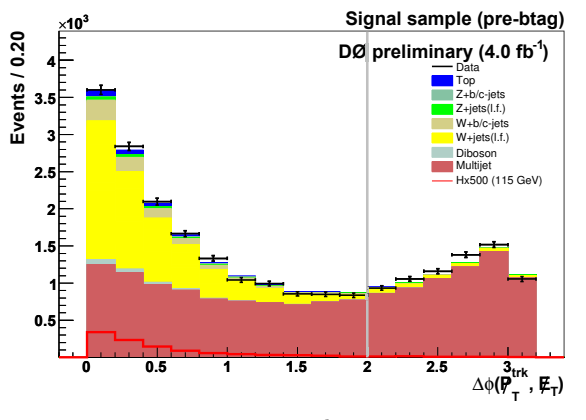

(b) $\Delta \phi\left(\not p_{\mathrm{T}}^{\mathrm{trk}}, \mathbb{E}_{\mathrm{T}}\right)$

(d) legend

Figure 4.3: (a) (b) and (c) show the selection variables used to select the QCD and $\mathrm{MC}$ control samples. The vertical lines depict the values where cuts are placed. These distributions in the signal sample are corrected for QCD and MC reweighting, as discussed in Section 4.3.1 and Section 4.3.4. Depicted are type 2 tau events, but type 1 tau events have similar distributions. A magnified legend $(\mathrm{d})$ is also shown. Note that the signal is multiplied with a factor 500 , to be able to see its shape in the same graph as the backgrounds. 


\begin{tabular}{lc}
\hline Tau type & MC sideband reweighting \\
\hline Tau type 1 & $0.69 \pm 0.08$ \\
Tau type 2, $\mathrm{NN}_{e}<0.5$ & $0.80 \pm 0.09$ \\
Tau type 2, $\mathrm{NN}_{e}>0.5$ & $0.97 \pm 0.06$ \\
\hline
\end{tabular}

Table 4.9: tau-type-dependent MC-sideband factors.

factor required in the MC. It is therefore also a test of how well tau-identification corrections, taggability and vertex-confirmation scale factors are parameterized, when compared to data. Both tau type 1 and 2 candidate events in Figure 4.4 show a good agreement in the MC control sample using the common correction factors alone. The measured data/MC ratio is $0.987 \pm 0.023$, so no reweighting is applied for the $\mathrm{NN}_{\tau}>0.9 \mathrm{MC}$.

\subsubsection{Sideband Region of the MC Sample}

As mentioned in Section 4.2.5, the multijet contribution is estimated by subtracting $\mathrm{MC}$-generated events from measured data in the sideband region, $0.3<$ $\mathrm{NN}_{\tau}<0.7$. This is also done for the $\mathrm{MC}$ control sample, hence with $\mathbb{E}_{\mathrm{T}}>$ $80 \mathrm{GeV}$.

Although statistical fluctuations could produce fewer data than MC background events in a certain bin, statistically significant data deficits are not possible as this would imply a negative multijet contribution. Most variables do not give such an unphysical result, except for $A$ and $m_{\mathrm{T}}(W)$, which are plotted in Figure 4.5

The regions $A>0$ and $m_{\mathrm{T}}(W)$ around the $W$ mass peak clearly show fewer data than MC-background events for tau type 1 and tau type 2 with $\mathrm{NN}_{e}<0.5$. This is not possible, given that the multijet background has to remain positive, so we conclude that the MC is overestimated in the sideband region. To solve this, we apply a tau-type-dependent factor to the MC backgrounds in the sideband region of every sample. These factors are derived by the ratio of data and MC integrals over the regions $A>0$ and $60 \mathrm{GeV}<m_{\mathrm{T}}(W)<160 \mathrm{GeV}$ in the $\mathrm{MC}$ control sample. In Table 4.9 the resulting MC-sideband reweighting factors are listed.

\subsubsection{Treatment of Bins with Negative Content}

Statistical fluctuations can still lead to bins with a negative multijet contribution. We use a Bayesian method illustrated in Figure 4.6 to deal with this. Initially, the red 'before correction' probability density function (pdf) describes the multijet 


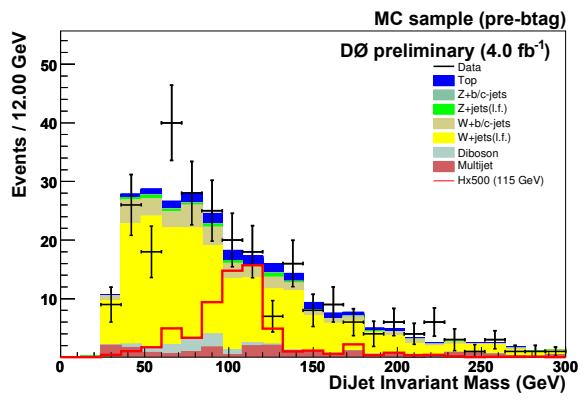

(a) dijet mass for type 1 taus

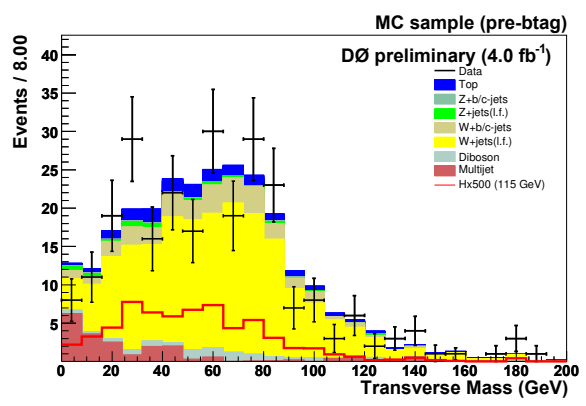

(c) $m_{\mathrm{T}}(W)$ for type 1 taus

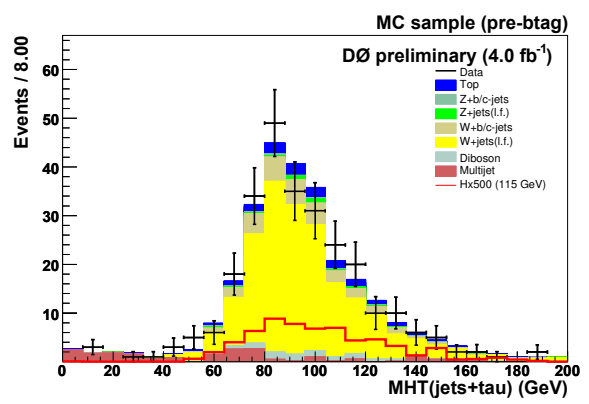

(e) H $_{\mathrm{T}}$ (jets + tau) for type 1 taus

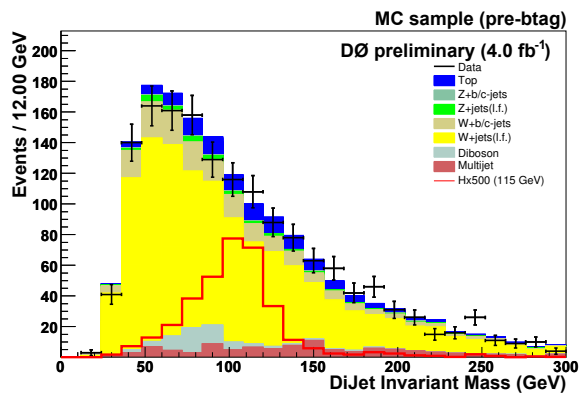

(b) dijet mass for type 2 taus

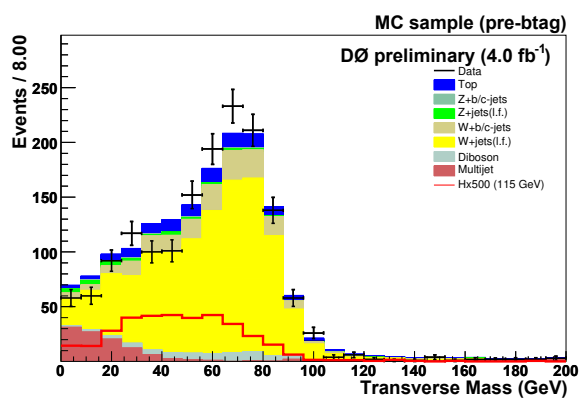

(d) $m_{\mathrm{T}}(W)$ for type 2 taus

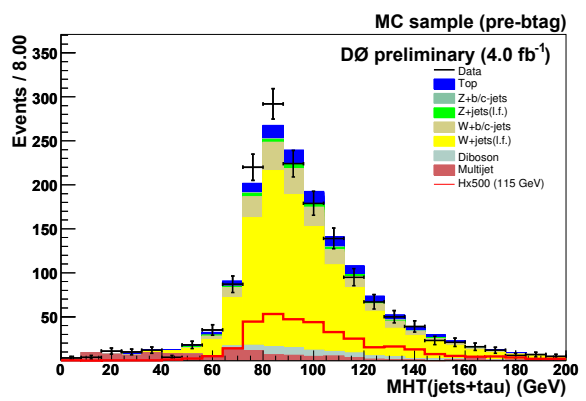

(f) $\not I_{\mathrm{T}}($ jets + tau $)$ for type 2 taus

Figure 4.4: data versus background comparisons for the MC control sample. For a magnified legend, see Figure 4.3(d). 


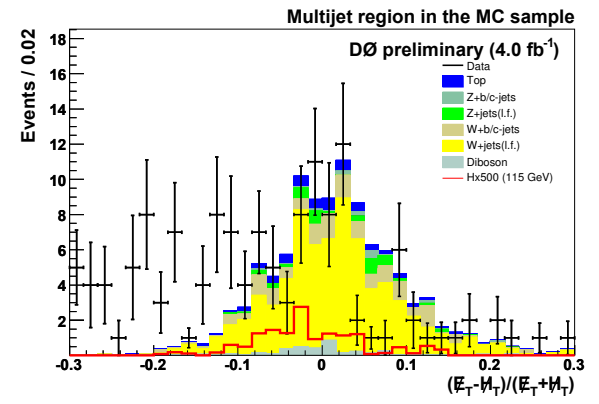

(a) $A$ for type 1 taus

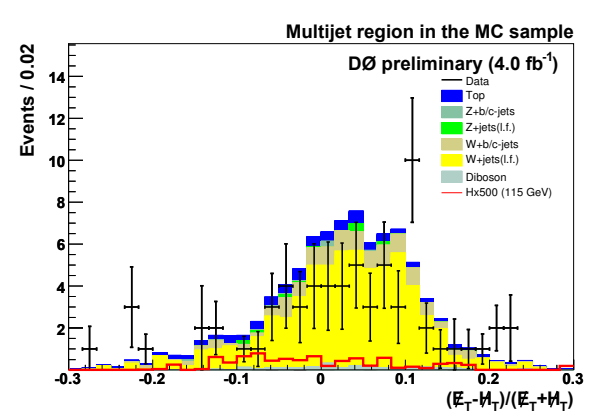

(c) A for type 2, low NNe taus

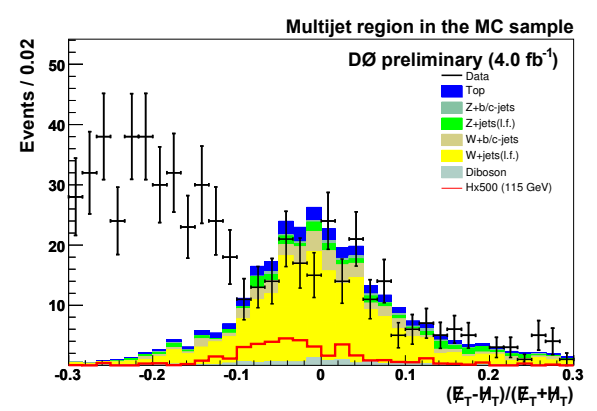

(e) $A$ for type 2 , high NNe taus

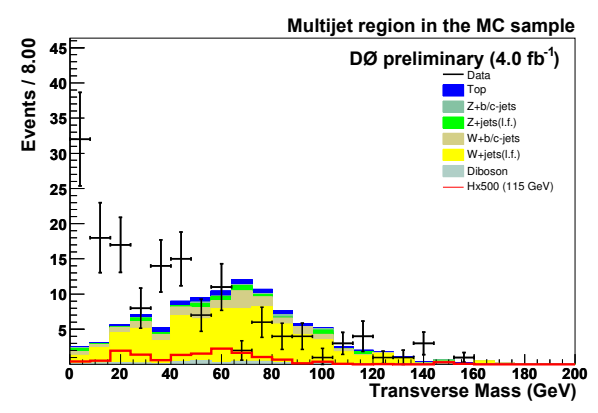

(b) $m_{\mathrm{T}}(W)$ for type 1 taus

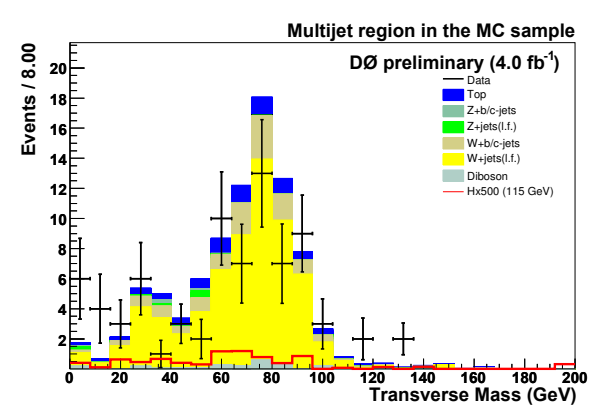

(d) $m_{\mathrm{T}}(W)$ for type 2 , low NNe taus

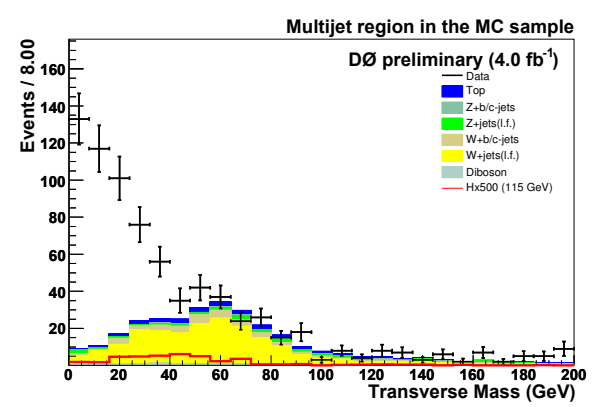

(f) $m_{\mathrm{T}}(W)$ for type 2 , high NNe taus

Figure 4.5: $A$ and $m_{\mathrm{T}}(W)$ distributions in the sideband of the MC sample. The multijet background is defined as the difference between the data and the Monte-Carlo-generated backgrounds. For a magnified legend, see Figure $4.3(\mathrm{~d})$. 


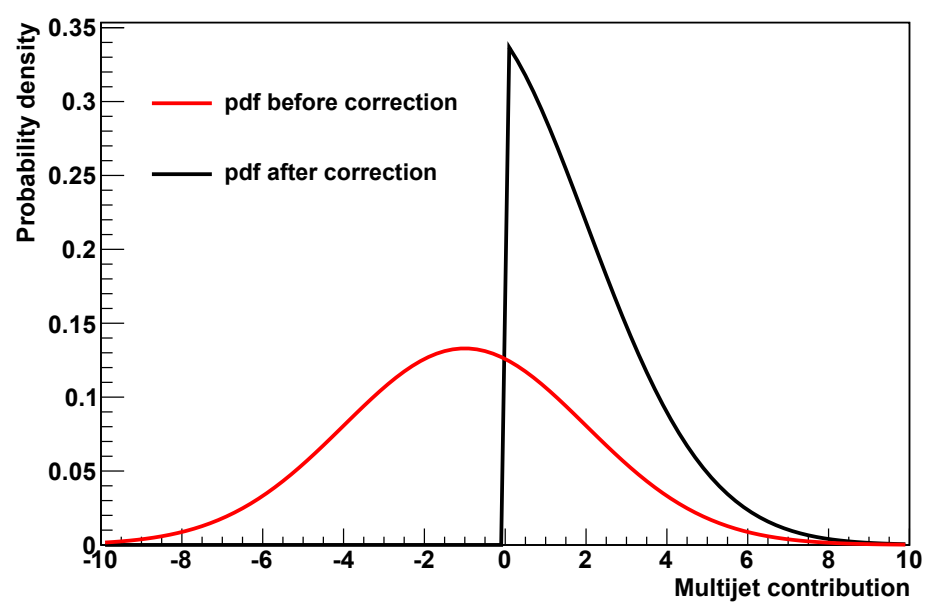

Figure 4.6: example of the method to treat the negative multijet contribution in a certain bin. In this case, the multijet estimation $-1 \pm 3$ leads to the corrected estimation $2.1 \pm 1.6$.

estimate in a certain bin, with its mean and uncertainty. Because we have the prior knowledge that a background can not be negative, we arrive at the black curve. Finally, we need to translate the pdf into an expected value of the multijet contribution for this bin, with uncertainty. This is done by taking the mean and RMS of the new pdf.

This correction is applied to every bin, so also slightly positive bins with large uncertainty are affected.

Because this method only leads to upward corrections to the multijet contribution, the resulting distribution is scaled down to offset the upward bias. To be precise, the scaling is applied mostly to bins adjacent to the corrected bin, by scaling bin $i$ with factor

$$
F_{i}=1-A \exp \left(-\frac{1}{2}(i-j)^{2}\right)
$$

where bin $j$ is the corrected bin and $A$ is chosen such that the total downward scaling equals the upward correction of bin $j$. This procedure ensures that a multijet distribution fluctuating around zero will result in a much flatter distribution, which is close to (but above) zero. 


\subsubsection{QCD Control Sample}

In a similar way to the MC control sample, a QCD control sample is selected to check whether the multijet prediction models the data well. A sample with few MC-generated events is acquired by demanding $\not_{\mathrm{T}}^{\mathrm{SIG}}<3.5$ and $\Delta \phi\left(\not \phi_{\mathrm{T}}^{\mathrm{trk}}, \mathbb{E}_{\mathrm{T}}\right)>$ 2.0. Both are useful variables for separating those events that have $\mathbb{E}_{\mathrm{T}}$ from neutrinos from those that have fake $\mathbb{E}_{\mathrm{T}}$ due to mismeasurements. The missingtransverse-energy significance, $\mathbb{E}_{\mathrm{T}}^{\mathrm{SIG}}$, does this by taking into account the uncertainty on the $\mathbb{E}_{\mathrm{T}}$, as is explained in Section 3.9. The angle between the direction of the $\not p_{\mathrm{T}}$ from tracks and the $\mathbb{E}_{\mathrm{T}}, \Delta \phi\left(\not_{\mathrm{T}}^{\mathrm{trk}}, \mathbb{E}_{\mathrm{T}}\right)$, is peaked around zero for real missing-energy events, but more spread out for multijet events. The distributions of these variables can be seen in Figure 4.3(a) and Figure 4.3(b)

\section{Flat Reweighting}

First, the overall multijet normalization factor, $C$ in Equation 4.2), is determined by dividing the data minus MC backgrounds in the signal region by the data minus MC backgrounds in the sideband region. This yields $C=0.28$ with a statistical uncertainty of 0.004 . The systematical uncertainty is larger, as explained in Section 5.2.1.

The resulting distributions are found to agree well for jet-based variables, but have significant deviations for tau variables, especially those that are important in the tau neural network. This is because we are comparing a control sample with $\mathrm{NN}_{\tau}>0.9$, to a multijet model with $0.3<\mathrm{NN}_{\tau}<0.7$.

Figure 4.7 shows the data and background distributions using a flat multijet normalization factor. It is clear that the variables $p_{\mathrm{T}}(\tau)$ and $\eta_{\mathrm{d}}(\tau)$ are mismodeled.

\section{Reweightings in $p_{\mathrm{T}}(\tau)$ and $\eta_{\mathrm{d}}(\tau)$}

To improve the multijet modeling in tau variables such as $p_{\mathrm{T}}(\tau)$ and $\eta_{\mathrm{d}}(\tau)$, the multijet contribution is reweighted. This requires an additional reweighting, which is a function of $p_{\mathrm{T}}(\tau)$ and $\eta_{\mathrm{d}}(\tau)$. It is found that the $p_{\mathrm{T}}(\tau)$ reweighting improves many tau variables as it is directly correlated with them. The $\eta_{\mathrm{d}}(\tau)$ reweighting is also necessary as this distribution is an important discriminating variable in the hadronic tau neural network. The $p_{\mathrm{T}}(\tau)$ and $\eta_{\mathrm{d}}(\tau)$ reweighting factors are derived using the type 2 sample, because it has the highest statistics, and applied to both type 1 and 2 tau candidate events.

The $p_{\mathrm{T}}(\tau)$ reweighting is fitted using a 4th order polynomial function, of which the result is shown in Figure $4.8(\mathrm{a})$. The $\eta_{\mathrm{d}}(\tau)$ distribution is rather difficult to fit and so a bin-by-bin reweighting is chosen, using the histogram shown in 
Figure 4.8(b) The $p_{\mathrm{T}}(\tau)$ reweighting is applied simultaneously with the $\eta_{\mathrm{d}}(\tau)$ reweighting.

Distributions after $p_{\mathrm{T}}(\tau)$ and $\eta_{\mathrm{d}}(\tau)$ reweightings are shown in Figure 4.9. It can be seen that there still is no perfect match between data and background in the variables $p_{\mathrm{T}}(\tau)$ and $\eta_{\mathrm{d}}(\tau)$ of tau type 2 events. This is caused by the simultaneous reweighting. In tau type 1 events, the same reweighting as determined on the sample with type 2 taus seems to work quite well.

\subsubsection{Summary of Reweightings for the QCD Estimation}

To recapitulate the past sections, a short summary of the discussed reweightings to the multijet estimation is given here.

To estimate the multijet background contribution in the signal region $\mathrm{NN}_{\tau}>$ 0.9 , a sideband region with $0.3<\mathrm{NN}_{\tau}<0.7$ is formed, where the difference between data and MC backgrounds is used as a model for the multijet background. We concluded in Section 4.3 .2 that the MC is overestimated in the sideband region of all samples. Therefore, the tau-type-dependent reweighting of Table 4.9 is applied to the Monte Carlo in the sideband regions of all samples.

In the QCD control sample of Section 4.3.4, we determined first the flat reweighting from sideband multijet to signal region multijet. Because many taubased variables were ill-described, an additional reweighting was performed as a function of $p_{\mathrm{T}}(\tau)$ and $\eta_{\mathrm{d}}(\tau)$.

The updated equation to determine the QCD in a sample now reads

$$
\mathrm{QCD}^{\text {sample }}=C \times D\left(p_{\mathrm{T}}(\tau), \eta_{\mathrm{d}}(\tau)\right) \times\left(\text { Data }_{\mathrm{sb}}^{\text {sample }}-B \times \mathrm{MC}_{\mathrm{sb}}^{\text {sample }}\right),
$$

where $C=0.28 \pm 0.004 . B$ and $D\left(p_{\mathrm{T}}(\tau), \eta_{\mathrm{d}}(\tau)\right)$ are described in Table 4.9 and Figure 4.8 respectively.

The resulting multijet distributions in the QCD control sample are shown in Figure 4.9

\subsection{Signal Sample}

The next step is to form the sample in which the final stages of the Higgs search will take place, by further selection cuts that reduce the multijet background. Before this is done, we check the agreement between data and background at the stage where the background is still dominated by multijet events.

Distributions of the dijet invariant mass, the $m_{\mathrm{T}}(W)$ and the $\not_{\mathrm{T}}$ are shown in Figure 4.10 for both tau types. Many of the variables are well modeled, except for the $W$-transverse-mass distribution. These discrepancies likely arise from QCD mismodeling and are expected to reduce after multijet-removing cuts are placed. 


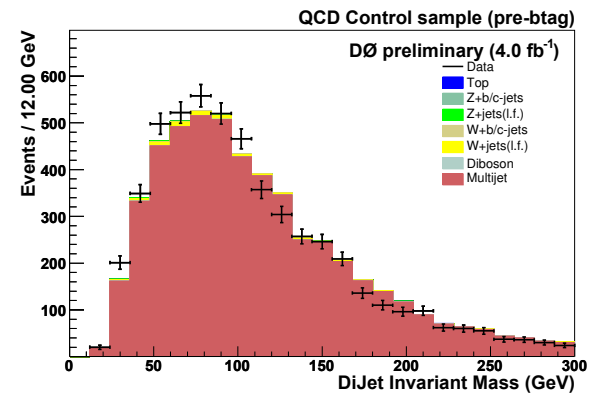

(a) dijet mass

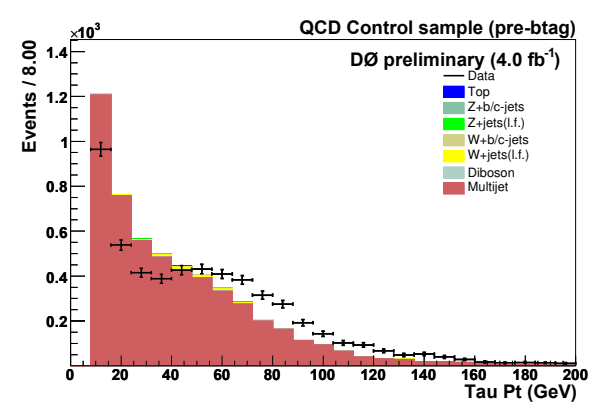

(c) $p_{\mathrm{T}}(\tau)$

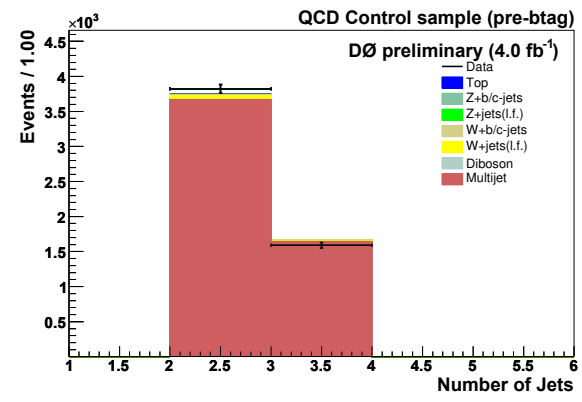

(b) jet multiplicity

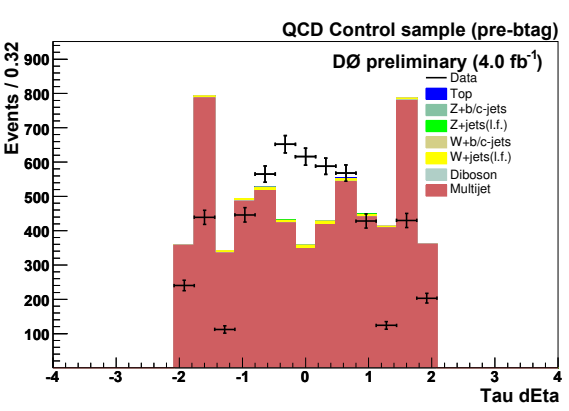

(d) $\eta_{\mathrm{d}}(\tau)$

Figure 4.7: data versus background comparisons for the QCD control sample with a flat normalization factor. For a magnified legend, see Figure $4.3(\mathrm{~d})$.

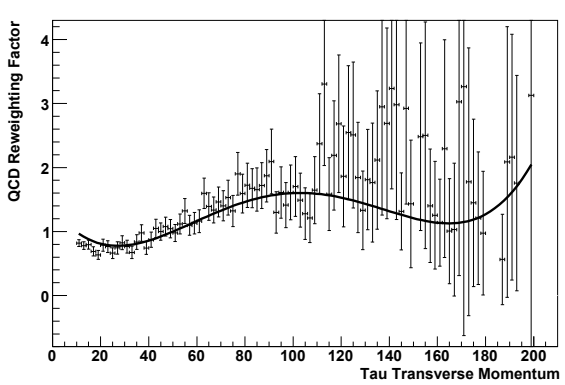

(a) $p_{\mathrm{T}}(\tau) \mathrm{RW}$

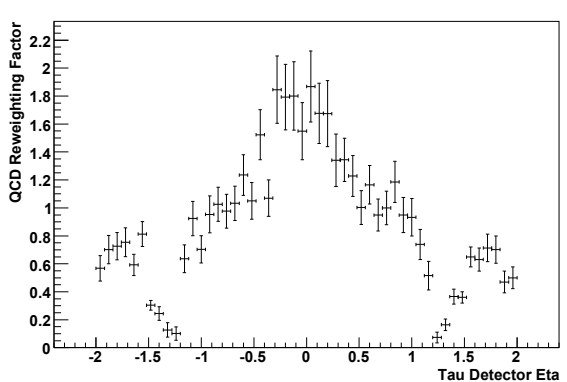

(b) $\eta_{\mathrm{d}}(\tau) \mathrm{RW}$

Figure 4.8: QCD reweightings in $p_{\mathrm{T}}(\tau)$ and $\eta_{\mathrm{d}}(\tau)$, derived from the distributions in Figure 4.7(c) and Figure 4.7(d). 


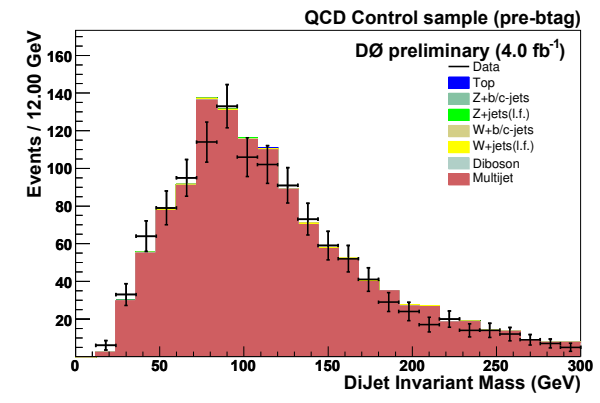

(a) dijet mass for type 1 tau events

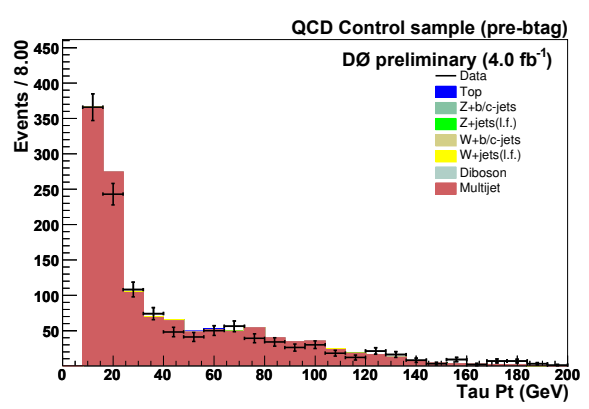

(c) $p_{\mathrm{T}}(\tau)$ for type 1 tau events

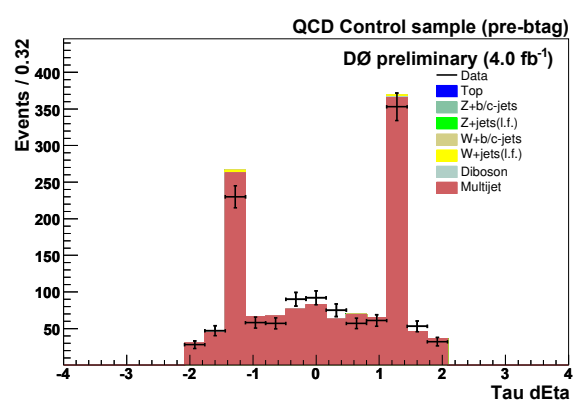

(e) $\eta_{\mathrm{d}}(\tau)$ for type 1 tau events

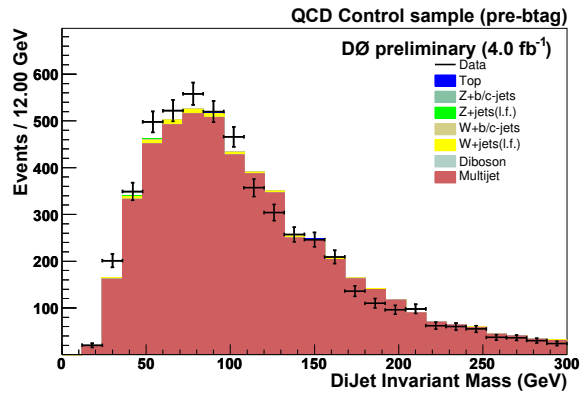

(b) dijet mass for type 2 tau events

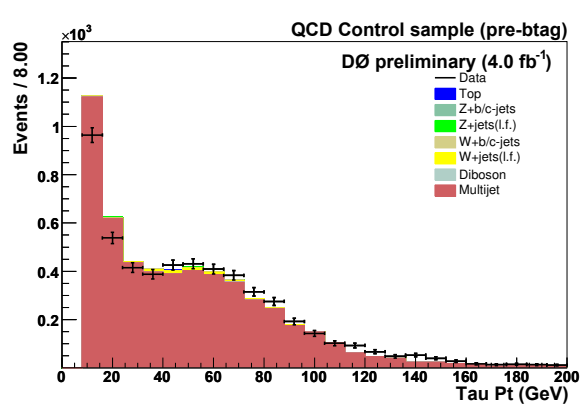

(d) $p_{\mathrm{T}}(\tau)$ for type 2 tau events

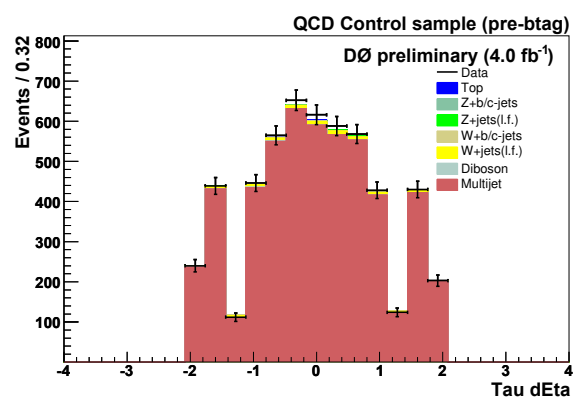

(f) $\eta_{\mathrm{d}}(\tau)$ for type 2 tau events

Figure 4.9: data versus background comparisons for the QCD control sample with a $p_{\mathrm{T}}(\tau)$ and $\eta_{\mathrm{d}}(\tau)$ dependent normalization factor. For a magnified legend, see Figure $4.3(\mathrm{~d})$. 


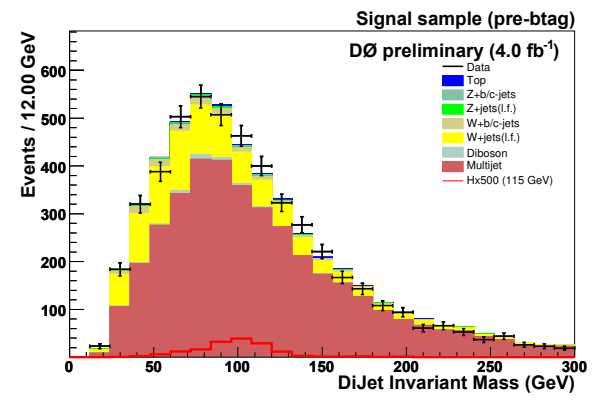

(a) dijet mass for type 1 taus

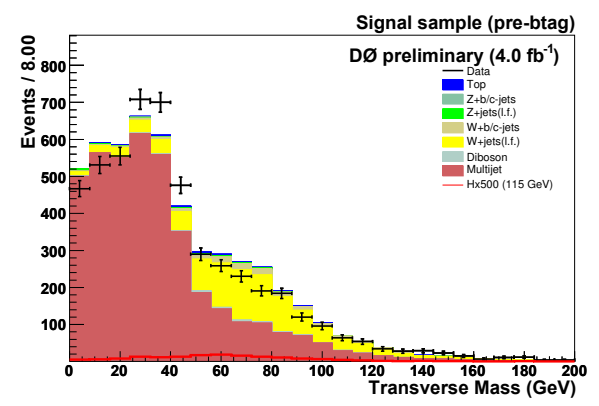

(c) $m_{\mathrm{T}}(W)$ for type 1 taus

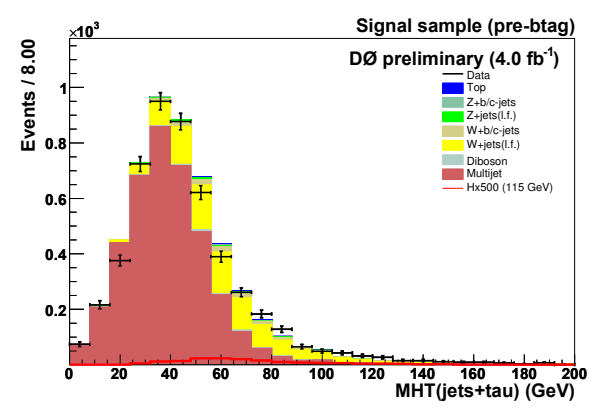

(e) H $_{\mathrm{T}}($ jets + tau $)$ for type 1 taus

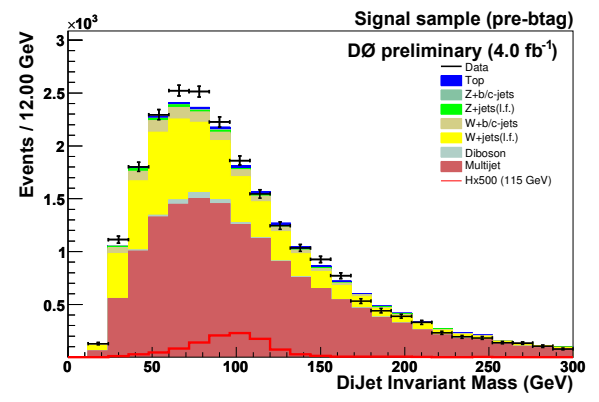

(b) dijet mass for type 2 taus

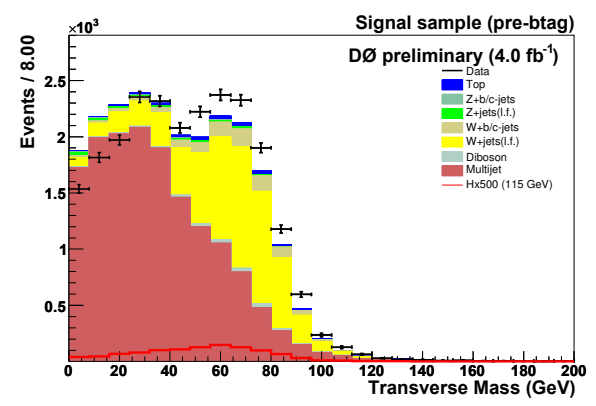

(d) $m_{\mathrm{T}}(W)$ for type 2 taus

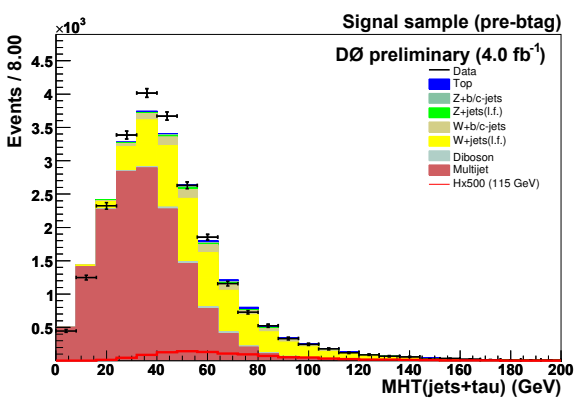

(f) $\not H_{\mathrm{T}}($ jets + tau $)$ for type 2 taus

Figure 4.10: data versus background comparisons for the signal region before signal sample cuts. For a magnified legend, see Figure $4.3(\mathrm{~d})$. 


\subsubsection{Further Selection Cuts}

Stricter cuts are placed on the signal sample to increase the signal purity. The cuts $\Delta \phi\left(\not p_{\mathrm{T}}^{\mathrm{trk}}, \mathbb{E}_{\mathrm{T}}\right)<2.0$ and $\mathbb{E}_{\mathrm{T}}^{\mathrm{SIG}}>4.5$ are found to be very efficient to remove much of the QCD background. They also provide independence from the QCD control samples, where the reweightings in $p_{\mathrm{T}}(\tau)$ and $\eta_{\mathrm{d}}(\tau)$ were determined.

At this point, comparisons between data and background are good tests of how well our reweighted QCD estimates and MC-sideband reweightings perform in the signal sample topology.

Generally, agreement is found to be good in this $W+$ jets dominated sample. For type 1 tau candidate events, the distributions in the signal sample can be seen in Figure 4.11. With the majority of events having a tau in the ICD, only a small number of taus exist in the central region of the detector. However, the modeling of key kinematic variables for this mixed sample is reasonably good.

Distributions of the signal sample for type 2 tau candidate events are shown in Figure 4.12. The agreement in tau type 2 candidate events after the selection cuts is once again good across many variables.

\subsubsection{Sideband Region after Further Selection}

The MC sideband reweighting discussed in Section 4.3.2 should be checked at this stage. In Figure 4.13 the variables $A$ and $m_{\mathrm{T}}(W)$ are shown for the sideband region after selection cuts and the reweighting of Table 4.9. These graphs confirm the need for the reweighting, because without it the regions $A>0$ and $m_{\mathrm{T}}(W)>$ $60 \mathrm{GeV}$ would have a negative multijet contribution.

Within statistical uncertainties, the distributions do not indicate that the multijet is structurally negative. They do show occasional bins with negative multijet content. In order to handle them properly in the signal region, we use the treatment discussed in Section 4.3.3.

\section{$4.5 \quad b$-Tagging}

The standard D $\varnothing$ b-tagging Neural Network [89, explained in Section 3.7, is used. The $b$-tagging is applied to the two highest- $p_{\mathrm{T}}$ jets in the events of the signal sample.

In a previous version of the $W H \rightarrow \tau \nu b \bar{b}$ analysis, using Run IIa data only, no multivariate analysis was performed; the dijet invariant mass was used as the input for further analysis [90]. Therefore, a tight $b$-tagging configuration was used: asymmetric tagging, where the event was required to contain a jet that passed the VeryTight operating point $\mathrm{NN}_{b}>0.85$, and another that passed the L3 operating point $\mathrm{NN}_{b}>0.25$. 


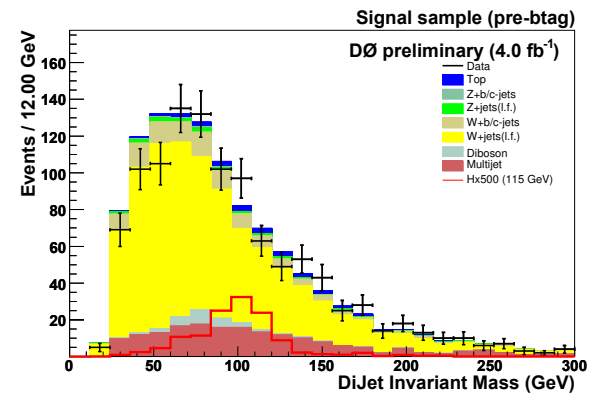

(a) dijet mass

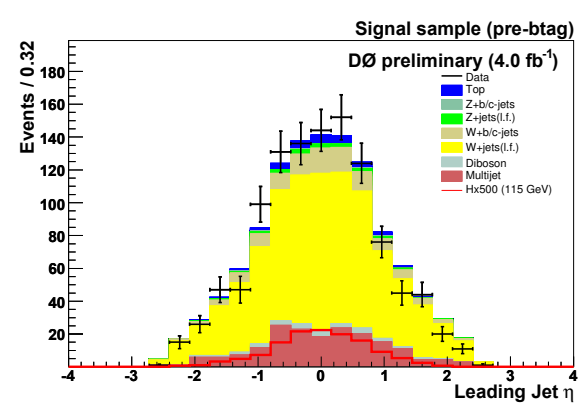

(c) $\eta(j e t 1)$

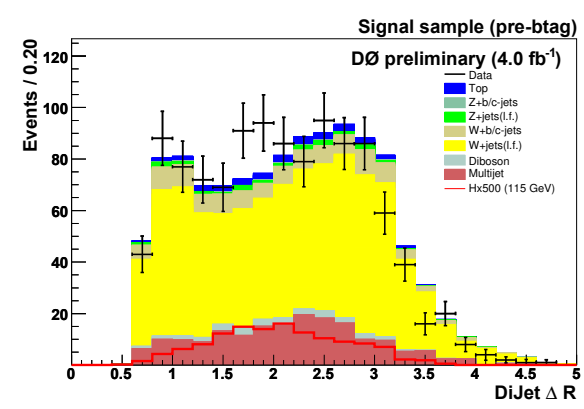

(e) $\Delta \mathrm{R}($ jet1,jet2)

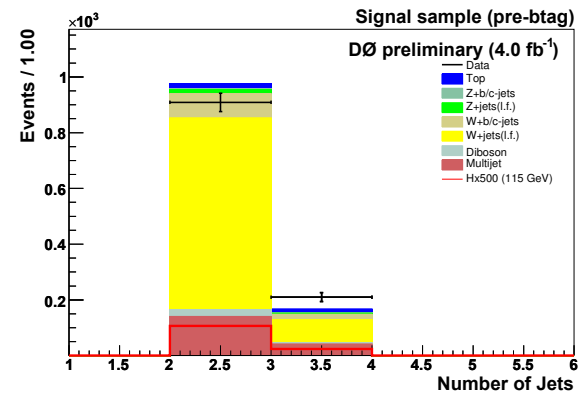

(b) jet multiplicity

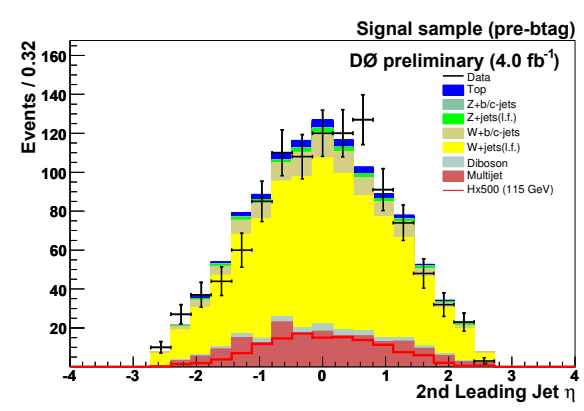

(d) $\eta(j e t 2)$

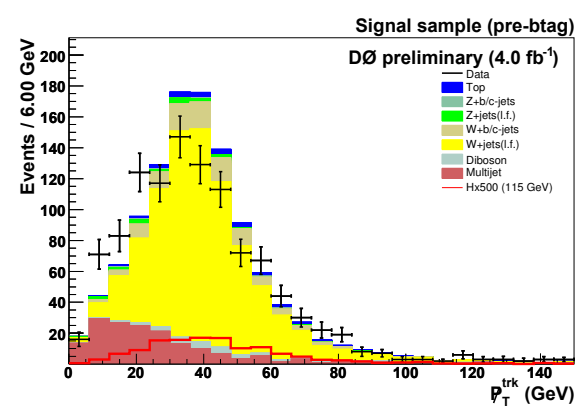

(f) $\not p_{\mathrm{T}}^{\mathrm{trk}}$

Figure 4.11: data versus background comparisons for the signal sample for type 1 tau candidate events. Continued on page 96 . 


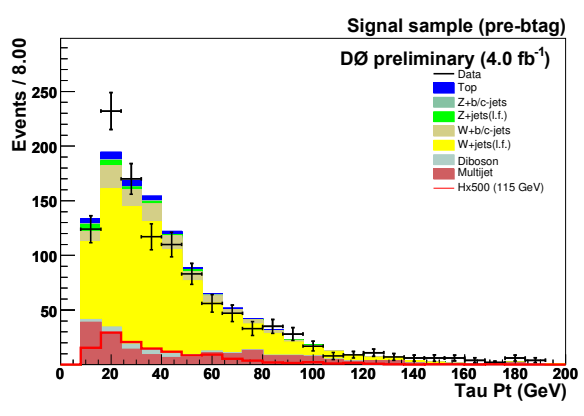

(g) $p_{\mathrm{T}}(\tau)$

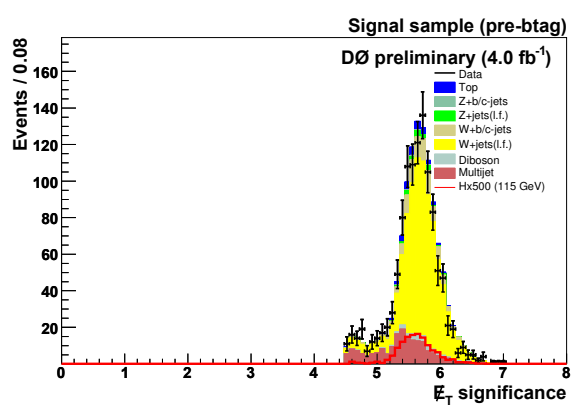

(i) $\mathbb{E}_{\mathrm{T}}^{\mathrm{SIG}}$

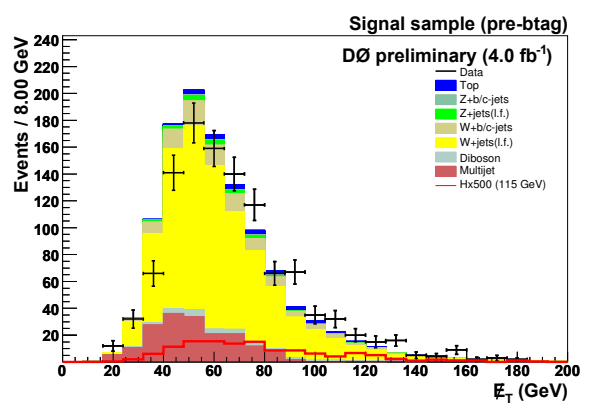

(k) $\mathbb{E}_{\mathrm{T}}$

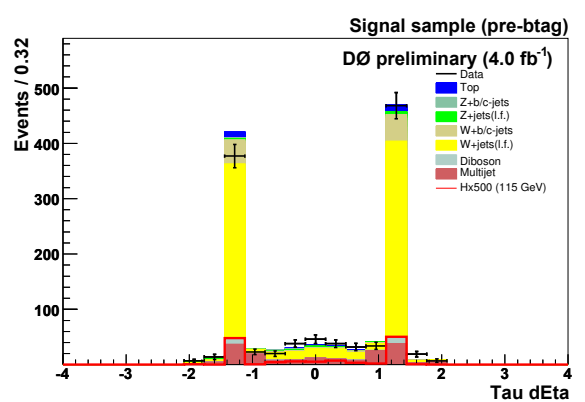

(h) $\eta_{\mathrm{d}}(\tau)$

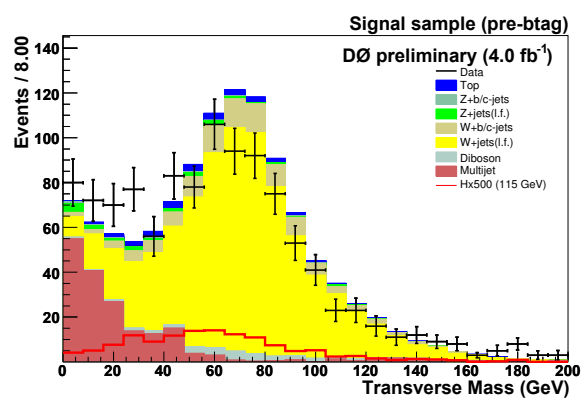

(j) $m_{\mathrm{T}}(W)$

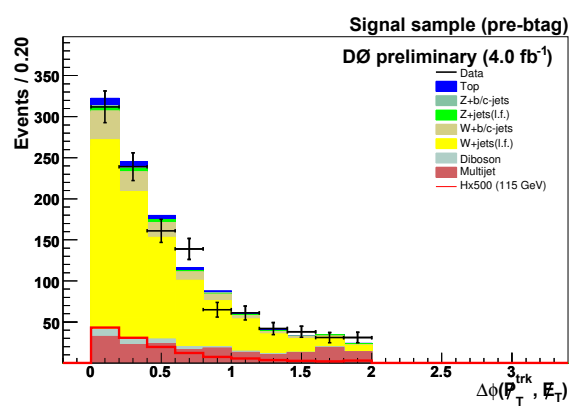

(l) $\Delta \phi\left(\not p_{\mathrm{T}}^{\mathrm{trk}}, \mathbb{E}_{\mathrm{T}}\right)$

Figure 4.11: data versus background comparisons for the signal sample for tau type 1 events. Continued from page 95 


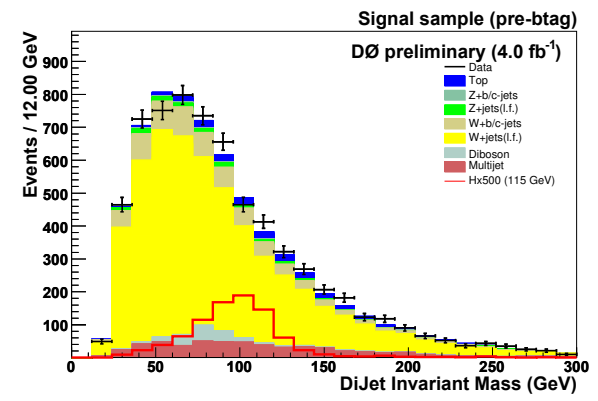

(a) dijet mass

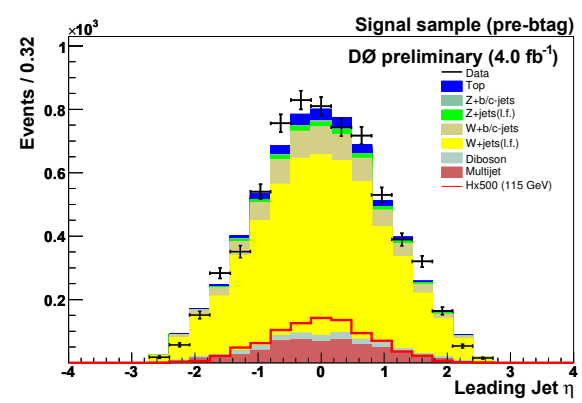

(c) $\eta($ jet 1$)$

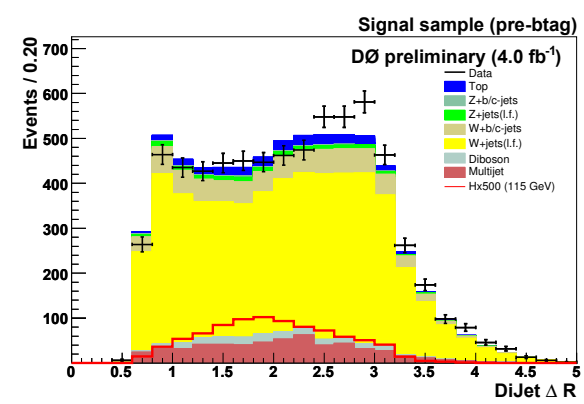

(e) $\Delta \mathrm{R}($ jet1,jet2)

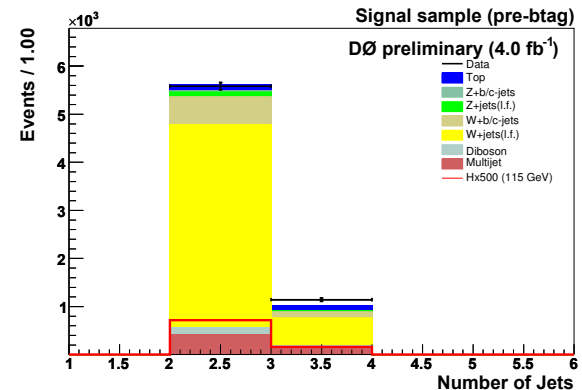

(b) jet multiplicity

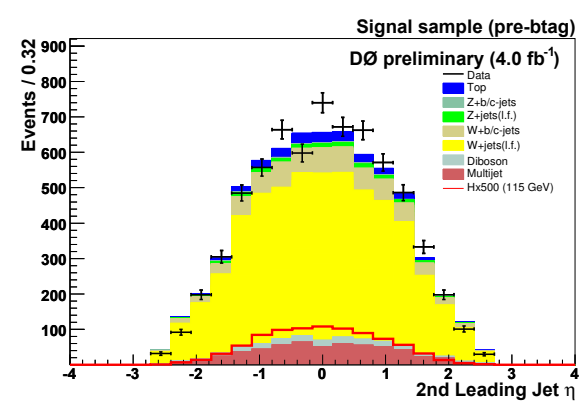

(d) $\eta($ jet2)

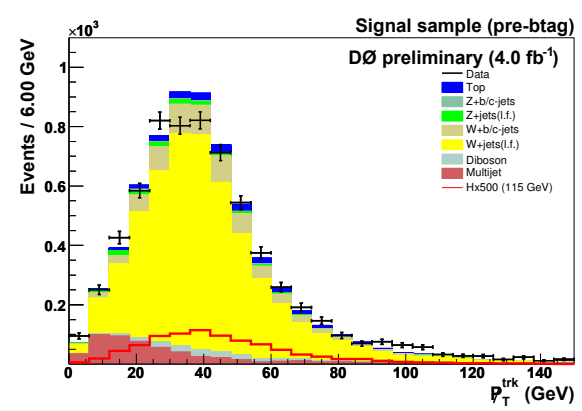

(f) $\not \mathrm{p}_{\mathrm{T}}^{\mathrm{trk}}$

Figure 4.12: data versus background comparisons for the signal sample for type 2 tau candidate events. Continued on page 98 . 


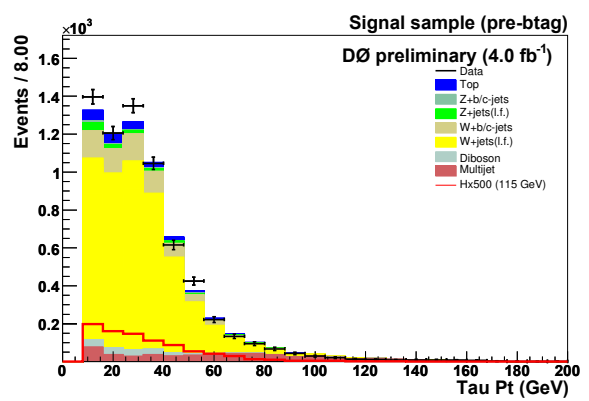

(g) $p_{\mathrm{T}}(\tau)$

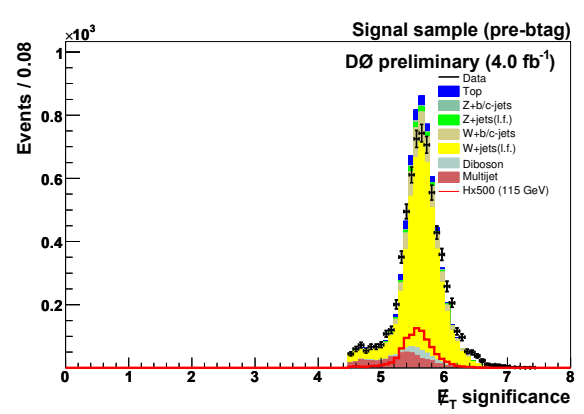

(i) $\mathbb{E}_{\mathrm{T}}^{\mathrm{SIG}}$

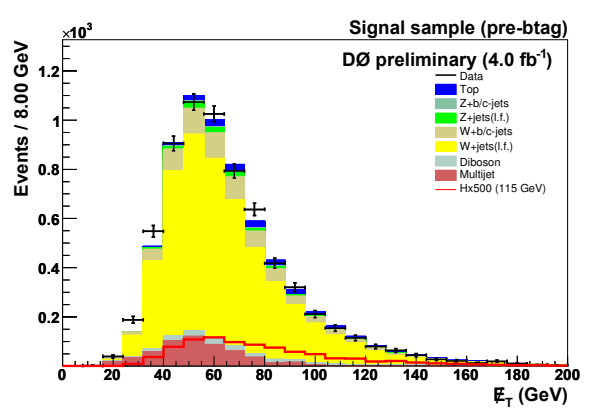

(k) $\mathbb{E}_{\mathrm{T}}$

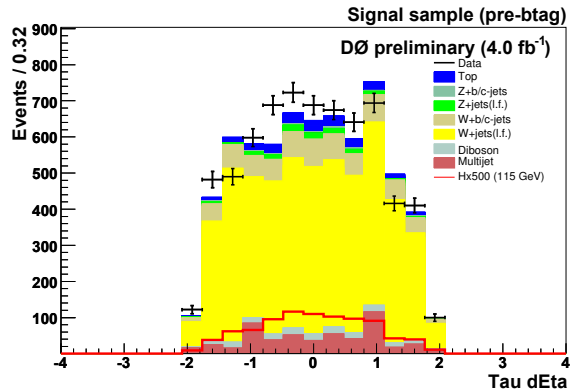

(h) $\eta_{\mathrm{d}}(\tau)$

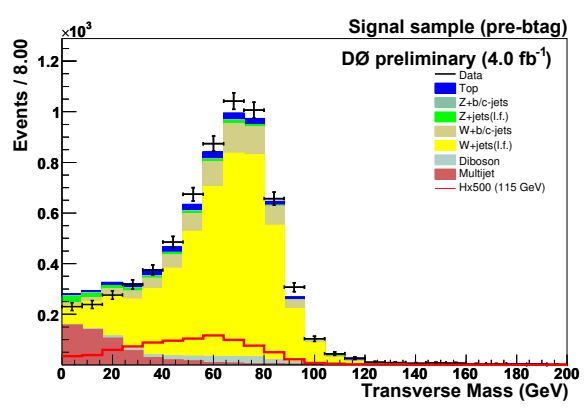

(j) $m_{\mathrm{T}}(W)$

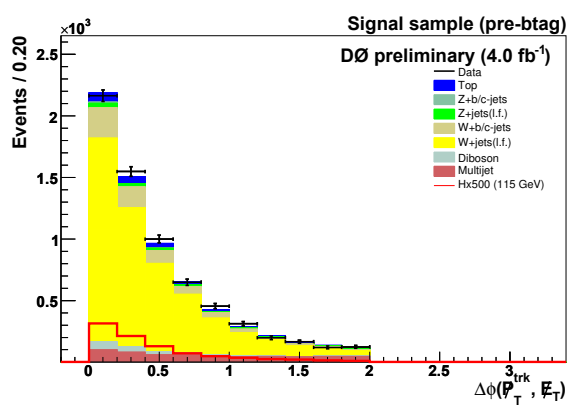

(l) $\Delta \phi\left(p_{\mathrm{T}}^{\mathrm{trk}}, \mathbb{E}_{\mathrm{T}}\right)$

Figure 4.12: data versus background comparisons for the signal sample for type 2 tau candidate events. Continued from page 97. 


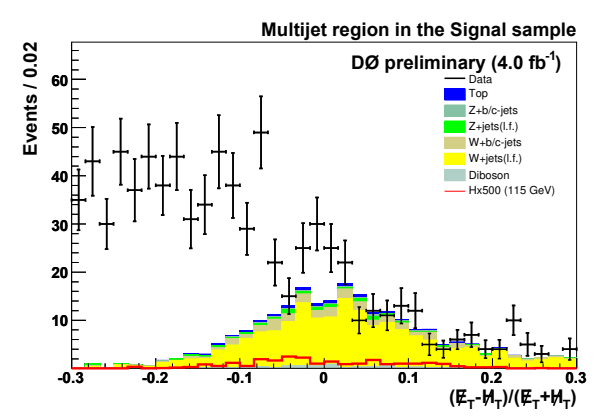

(a) $A$ for type 1 taus

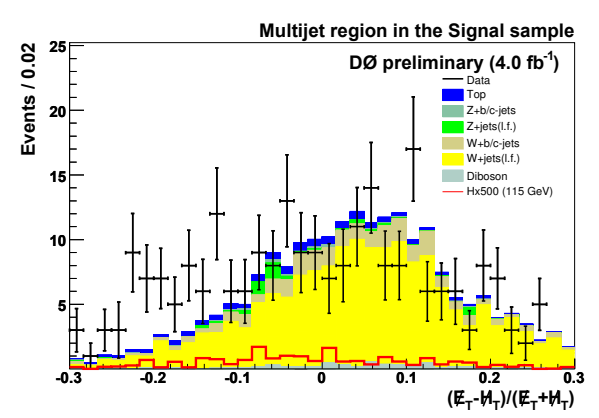

(c) A for type 2 , low $\mathrm{NNe}$ taus

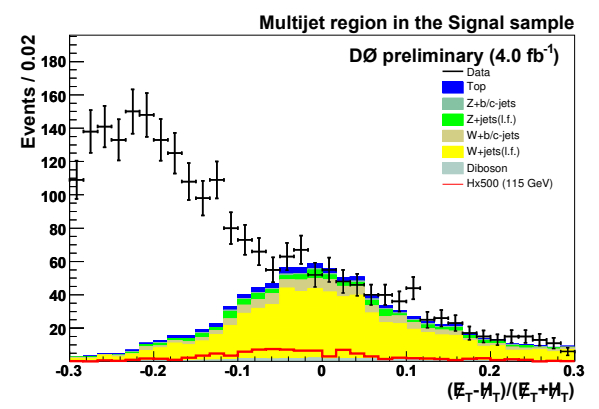

(e) $A$ for type 2 , high $\mathrm{NNe}$ taus

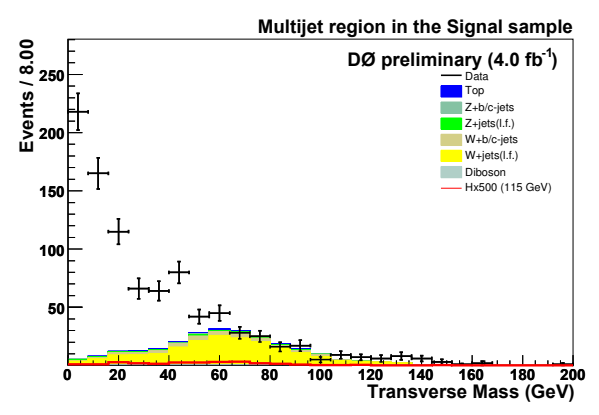

(b) $m_{\mathrm{T}}(W)$ for type 1 taus

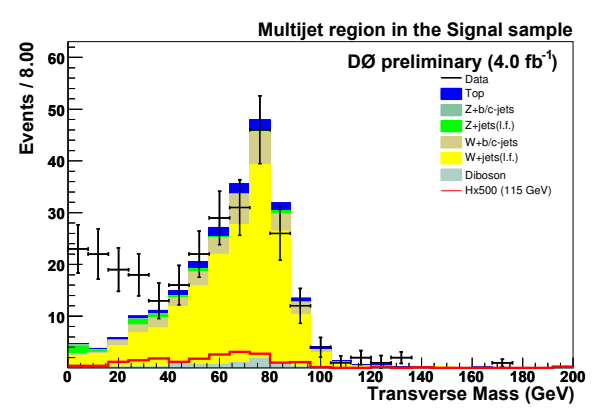

(d) $m_{\mathrm{T}}(W)$ for type 2 , low NNe taus

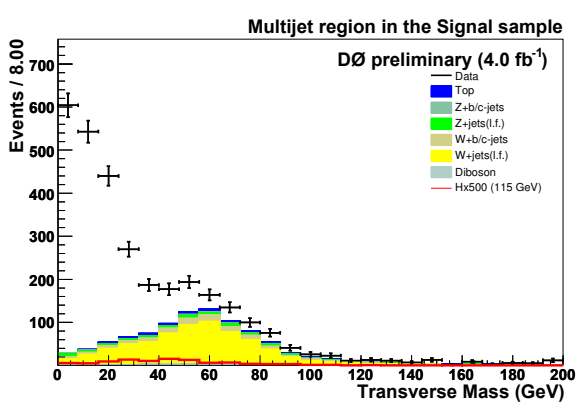

(f) $m_{\mathrm{T}}(W)$ for type 2 , high NNe taus

Figure 4.13: $A$ and $m_{\mathrm{T}}(W)$ distributions in the sideband of the signal sample after the MC sideband reweighting of Section 4.3.2. The multijet background is defined as the difference between the data and the Monte-Carlo-generated backgrounds. 


\begin{tabular}{lcccccccr}
\hline Sample & $W+$ jets & $Z+$ jets & Top & Diboson & QCD & Background & Signal & Observed \\
\hline Pretag & 7208 & 182.2 & 279.8 & 202.4 & 745 & $7775 \pm 117$ & 2.0 & 7844 \\
1-tag & 207.2 & 8.64 & 108.05 & 12.26 & 48.9 & $385.1 \pm 2.1$ & 0.791 & 380 \\
2-tag & 86 & 3.81 & 78.96 & 4.94 & 19.3 & $193 \pm 0.9$ & 0.918 & 190 \\
\hline
\end{tabular}

Table 4.10: event yields before and after $b$-tagging for the expected signal, the estimated background and the observed events. Only statistical uncertainties are quoted.

In this version of the analysis however, a multivariate technique is used, as will be discussed in Section 5.1. This makes it sensible to use much looser $b$-tagging requirements, because the rest of the event kinematics aid the discrimination between signal and background.

Two orthogonal $b$-tagged samples are created where the leading two jets in transverse momentum are considered. The 'TwoLoose' sample is defined as the set of events in which the leading two jets both pass the $\mathrm{NN}_{b}>0.25$ requirement. The 'OneTight' sample is defined as the set of events where one of the leading two jets passes the $\mathrm{NN}_{b}>0.85$ requirement and the other jet explicitly fails the $\mathrm{NN}_{b}>0.25$ requirement. These samples therefore contain no overlapping events and can be used as independent input channels for the hypothesis testing described in Chapter 5

The TwoLoose sample increases signal acceptance over the old asymmetric tagging condition, which allows the multivariate analysis more statistics for training and therefore for a better separation. As well as adding sensitivity to the search, the OneTight sample also helps to constrain the systematic uncertainties at the end of the analysis (see Section 5.4.1) as it has larger statistics than the TwoLoose sample.

In Table 4.10 the total number of events is shown for the final sample without $b$-tagging applied, for the OneTight (1-tag) sample and for the TwoLoose (2-tag) sample. The quoted numbers include tau types 1 and 2 , and both jet multiplicities.

\subsection{1 $b$-Tagging Scale Factors}

In Section 3.7 it is discussed how the TRFs are acquired. The MC-simulated events get a scale factor to correct for the higher tagging efficiency in MC than in data. For the TwoLoose and the OneTight samples, the scale factors are found in the following way.

In a $b$-jet sample with total number of events $N$ and a number of events with 
a loose tag $N_{\text {loose }}$, the number of events without loose tag $N_{\text {!loose }}$ is given by

$$
N_{\text {!loose }}=N-N_{\text {loose }}
$$

The TRF is then

$$
\mathrm{TRF}_{\text {loose }}=\frac{N_{\text {loose }}}{N},
$$

while the probability of finding no loose tag is given by

$$
\mathrm{TRF}_{! \text {loose }}=\frac{N ! \text { loose }}{N}=1-\frac{N_{\text {loose }}}{N}=1-\mathrm{TRF}_{\text {loose }}
$$

The scale factor that needs to be applied to the MC for a loose tag is given by

$$
S_{\text {loose }}=\frac{\mathrm{TRF}_{\text {loose }}^{\text {data }}}{\mathrm{TRF}_{\text {loose }}^{\mathrm{MC}}}
$$

and for no loose tag

$$
S_{\text {!loose }}=\frac{\mathrm{TRF}_{\text {!loose }}^{\text {data }}}{\mathrm{TRF}_{! \text {loose }}^{\mathrm{MC}}}=\frac{1-\mathrm{TRF}_{\text {loose }}^{\mathrm{MC}} S_{\text {loose }}}{1-\mathrm{TRF}_{\text {loose }}^{\mathrm{MC}}} .
$$

The same holds for events with a tightly tagged jet.

On MC events with $b$-jets in the TwoLoose sample a $S_{\text {loose }}\left(\right.$ jet1) $S_{\text {loose }}($ jet2) scale factor was applied, while the ones in the OneTight sample get the factor $S_{\text {tight }}\left(\right.$ jet1) $S_{\text {!loose }}($ jet2).

For a light jet sample, similar equations are true with NTRs instead of TRFs; light-jet MC events have scale factors applied with the TRFs replaced by NTRs.

\subsubsection{OneTight $b$-Tag Selection}

Data-background comparisons after the OneTight criterion is applied to the signal sample are shown in Figure 4.14 for tau type 1 events and in Figure 4.15 for tau type 2 events. As the hypotheses are going to be tested per jet multiplicity, the $b$-tagged plots are shown here exclusively for 2 and 3 jet events. Note that the Higgs-boson signal in these histograms is multiplied by a factor of 50 to make its shape visible. ${ }^{2}$

The event yields specified per tau type and jet multiplicity with one tight $b$-tag can be seen in Table 4.11 .

In the tau type 2 sample with 3 jets a data excess is visible, while the sample with 3 jets and a type 1 tau has fewer data events than expected. Both discrepancies are in the order of 1-2 times the statistical uncertainty. These fluctuations

\footnotetext{
${ }^{2}$ As opposed to the factor 500 used previously.
} 
are not unexpected for a total of 8 samples. They are reflected in the 3 -jet graphs of Figures 4.14 and 4.15 .

Because top events constitute the main background of the 3-jet samples, the data suggests the top cross section or selection efficiency was underestimated. If this is true, it should be covered by the systematic uncertainties on the top background estimates, which will be discussed in Section 5.2 .

The sample with the best signal over square root of background ratio $(s / \sqrt{b})$ is the one with 2 jets and tau type 2 . In this sample the $W+$ jets background dominates, while the sample with tau type 2 and 3 jets has relatively more top background events. In the more sparsely populated tau type 1 samples, the multijet background plays a non-negligible role, with again a large $W+$ jets (top) contribution for $2(3)$ jet events.

\subsubsection{TwoLoose $b$-Tag Selection}

In Figures 4.16 and 4.17 the data versus background comparisons are shown for the TwoLoose criterion with events of tau type 1 and tau type 2 , respectively.

The event yields of the samples with two loose $b$-tags can be seen in Table 4.12 . specified per tau type and jet multiplicity.

Again the sample with 2 jets and a tau of type 2 is the most populated sample, followed by the one with 3 jets and a type 2 tau. These are $W+$ jets and top dominated, respectively, like for the 1-tag samples. Especially the sample of tau type 2 events with 3 jets has multijet events as main background.

The $s / \sqrt{b}$ is better for the tau type 1 sample with 2 jets than for the tau type 2 sample with 3 jets; the signal is very comparable between these samples, but with about half the background, the first sample looks more promising for the separation of the signal and no-signal hypotheses. 


\begin{tabular}{lcccc}
\hline Sample & Type 2,2 jet & Type 2,3 jet & Type 1,2 jet & Type 1,3 jet \\
\hline Diboson & 8.63 & 1.9 & 1.408 & 0.316 \\
QCD & 23.7 & 8.3 & 13.1 & 3.86 \\
Top & 51.27 & 43.98 & 7.19 & 5.6 \\
$W+b / c$-jets & 97.5 & 19.15 & 14.22 & 2.77 \\
$W+$ jets & 55.44 & 8.07 & 8.98 & 1.13 \\
$Z+b / c$-jets & 4.11 & 1.13 & 0.62 & 0.176 \\
$Z+$ jets & 1.839 & 0.419 & 0.276 & 0.071 \\
Background & $242.4 \pm 1.2$ & $83 \pm 0.5$ & $45.8 \pm 0.6$ & $13.93 \pm 0.19$ \\
Observed & 232 & 99 & 40 & 9 \\
Signal & 0.549 & 0.145 & 0.0772 & 0.0198 \\
$s / \sqrt{b}$ & 0.0353 & 0.0159 & 0.0114 & 0.00531 \\
\hline
\end{tabular}

Table 4.11: event yields of the one- $b$-tag samples. The signal numbers are given for a Higgs boson mass of $115 \mathrm{GeV}$. The quoted uncertainties are statistical only.

\begin{tabular}{lcccc}
\hline Sample & Type 2,2 jet & Type 2,3 jet & Type 1,2 jet & Type 1,3 jet \\
\hline Diboson & 3.58 & 0.683 & 0.599 & 0.081 \\
QCD & 12.5 & 1.22 & 3 & 2.6 \\
Top & 44.22 & 25.5 & 6.02 & 3.21 \\
$W+b / c$-jets & 46.5 & 6.57 & 7.06 & 0.95 \\
$W+$ jets & 18.71 & 2.875 & 2.962 & 0.406 \\
$Z+b / c$-jets & 2.1 & 0.457 & 0.352 & 0.092 \\
$Z+$ jets & 0.569 & 0.132 & 0.082 & 0.0232 \\
Background & $128.1 \pm 0.6$ & $37.44 \pm 0.15$ & $20.07 \pm 0.18$ & $7.36 \pm 0.11$ \\
Observed & 117 & 44 & 23 & 6 \\
Signal & 0.695 & 0.1036 & 0.103 & 0.0159 \\
$s / \sqrt{b}$ & 0.0614 & 0.01693 & 0.023 & 0.00586 \\
\hline
\end{tabular}

Table 4.12: event yields of the two- $b$-tag samples. The signal numbers are given for a Higgs boson mass of $115 \mathrm{GeV}$. The quoted uncertainties are statistical only. 


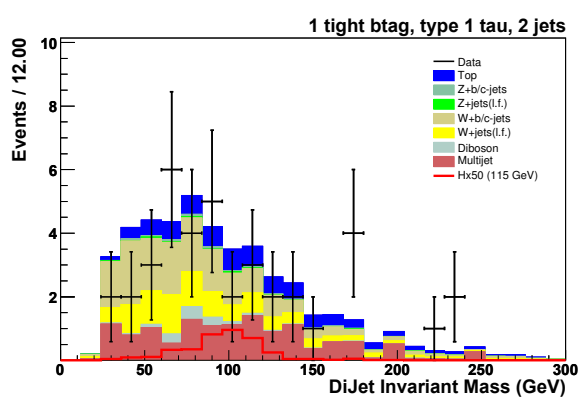

(a) dijet mass for 2 jet events

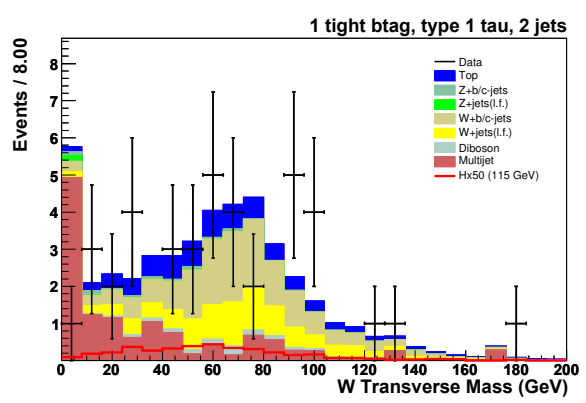

(c) $m_{\mathrm{T}}(W)$ for 2 jet events

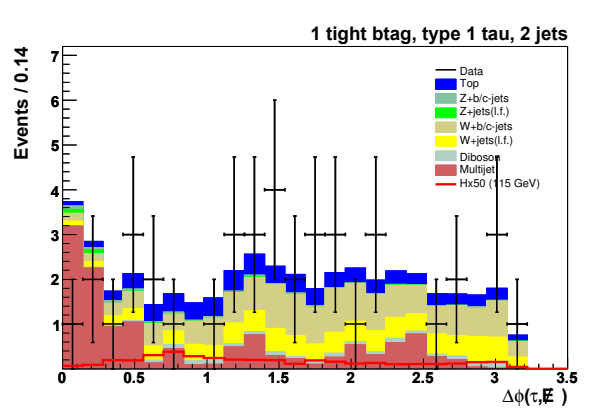

(e) $\Delta \phi\left(\tau, \mathbb{E}_{\mathrm{T}}\right)$ for 2 jet events

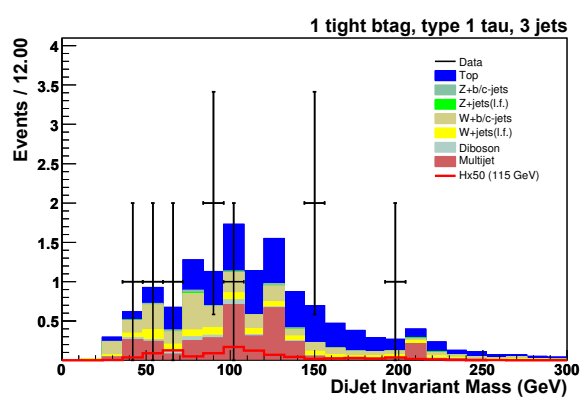

(b) dijet mass for 3 jet events

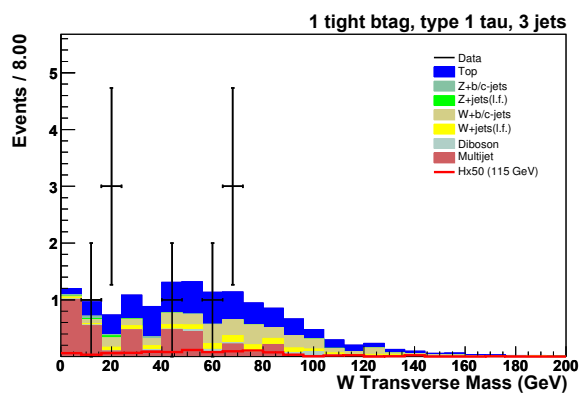

(d) $m_{\mathrm{T}}(W)$ for 3 jet events

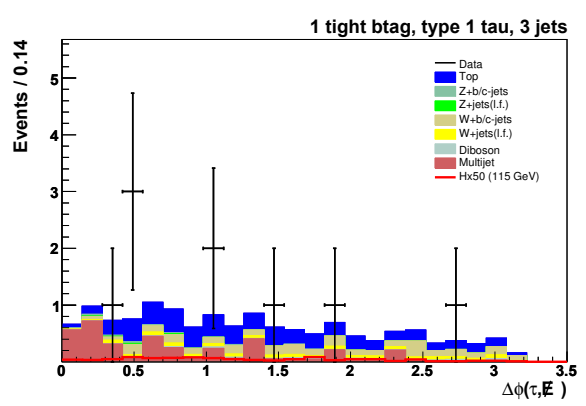

(f) $\Delta \phi\left(\tau, \mathbb{E}_{\mathrm{T}}\right)$ for 3 jet events

Figure 4.14: data versus background comparisons for the selection of tau type 1 events with one tight $b$-tag. 


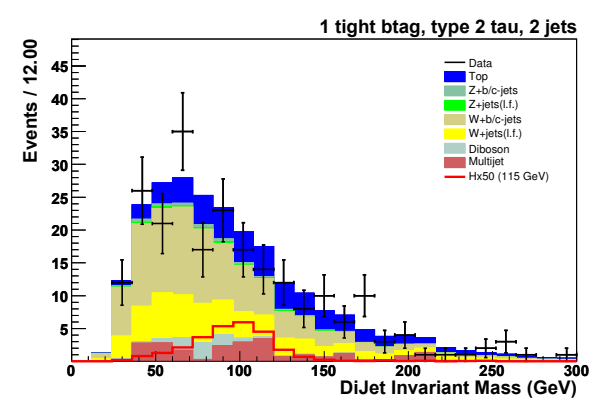

(a) dijet mass for 2 jet events

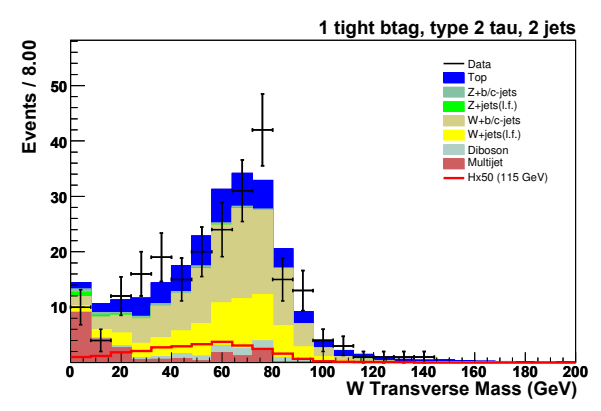

(c) $m_{\mathrm{T}}(W)$ for 2 jet events

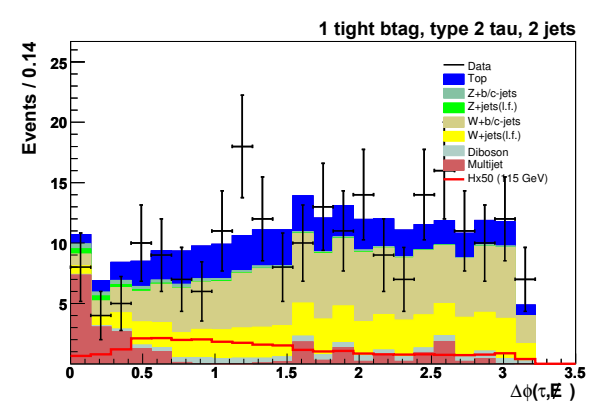

(e) $\Delta \phi\left(\tau, \mathbb{E}_{\mathrm{T}}\right)$ for 2 jet events

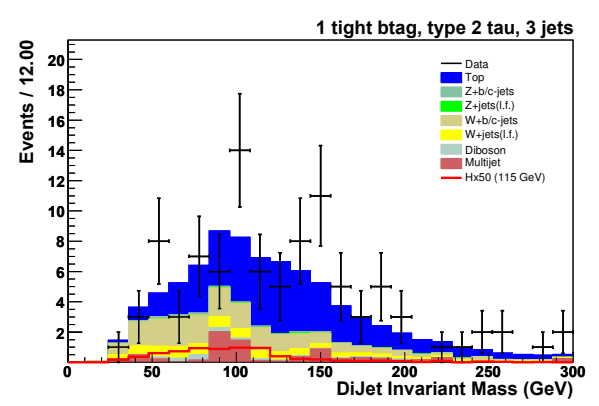

(b) dijet mass for 3 jet events

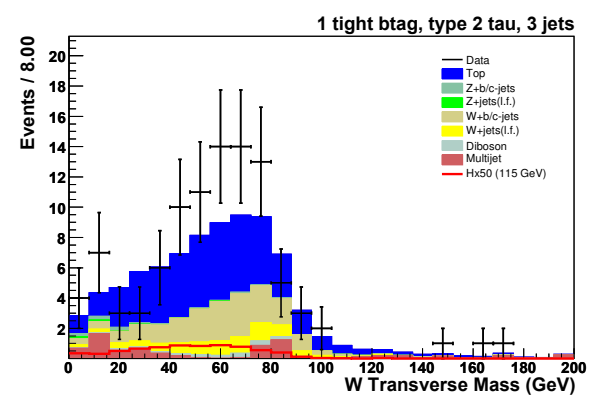

(d) $m_{\mathrm{T}}(W)$ for 3 jet events

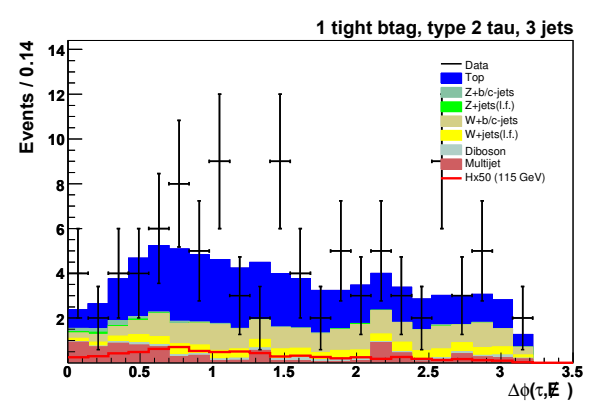

(f) $\Delta \phi\left(\tau, \notin_{\mathrm{T}}\right)$ for 3 jet events

Figure 4.15: data versus background comparisons for the selection of tau type 2 events with one tight $b$-tag. 


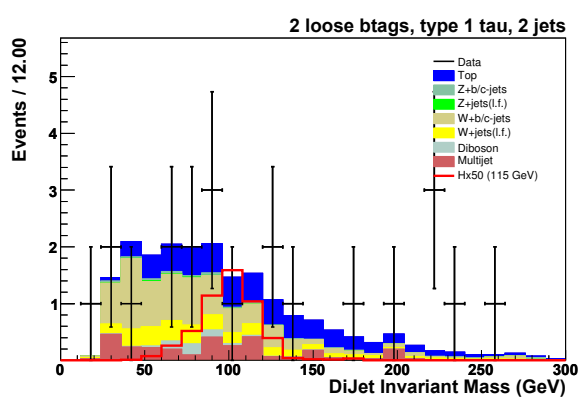

(a) dijet mass for 2 jet events

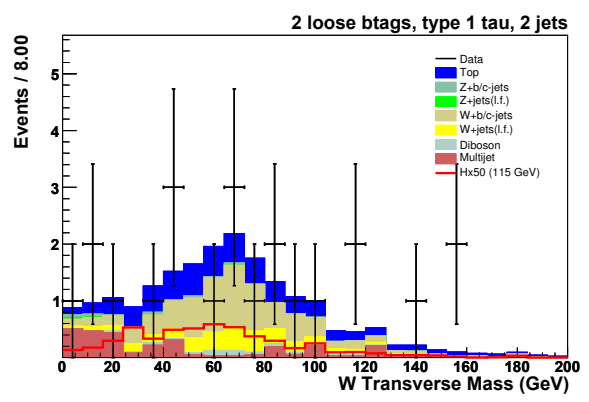

(c) $m_{\mathrm{T}}(W)$ for 2 jet events

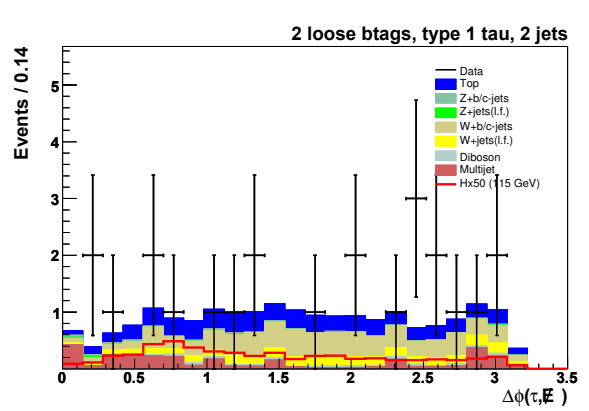

(e) $\Delta \phi\left(\tau, \notin_{\mathrm{T}}\right)$ for 2 jet events

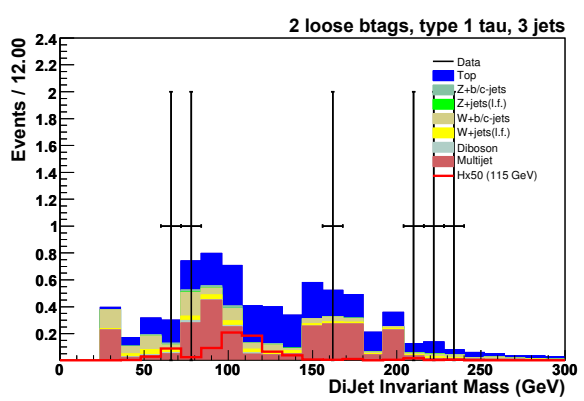

(b) dijet mass for 3 jet events

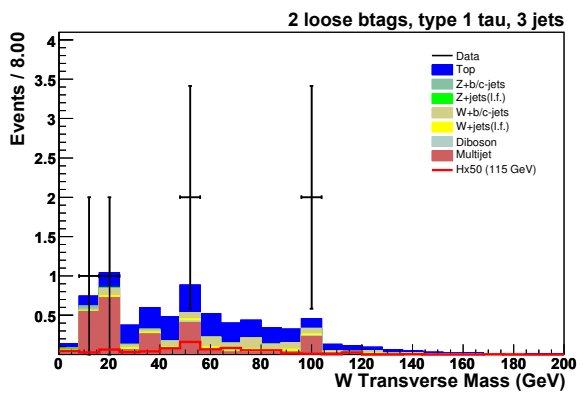

(d) $m_{\mathrm{T}}(W)$ for 3 jet events

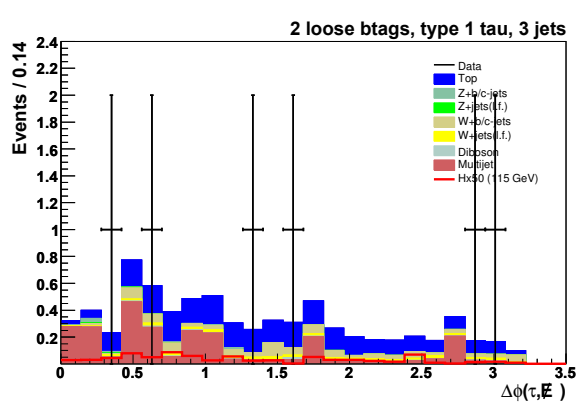

(f) $\Delta \phi\left(\tau, \mathbb{E}_{\mathrm{T}}\right)$ for 3 jet events

Figure 4.16: data versus background comparisons for the selection of tau type 1 events with two loose $b$-tags. 


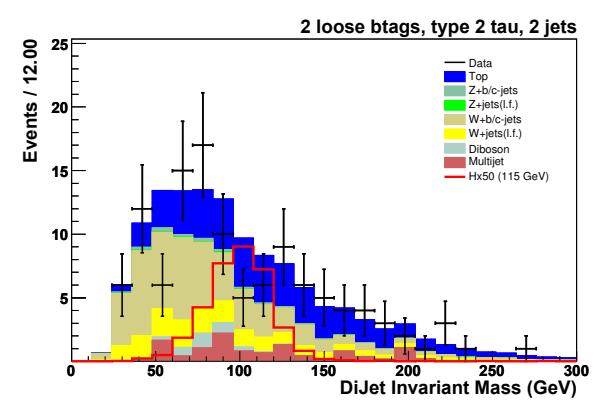

(a) dijet mass for 2 jet events

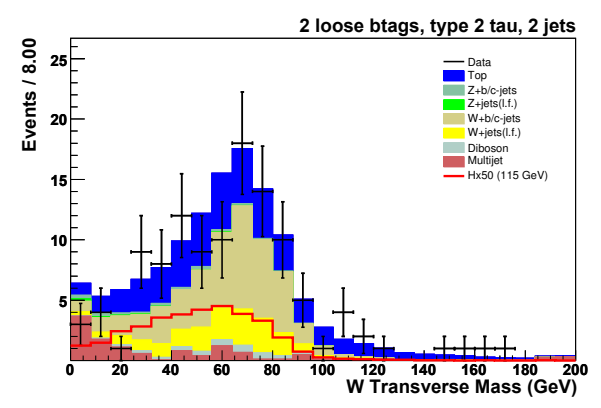

(c) $m_{\mathrm{T}}(W)$ for 2 jet events

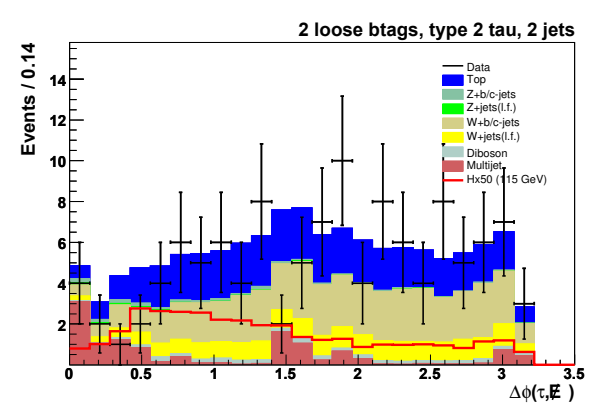

(e) $\Delta \phi\left(\tau, \mathbb{E}_{\mathrm{T}}\right)$ for 2 jet events

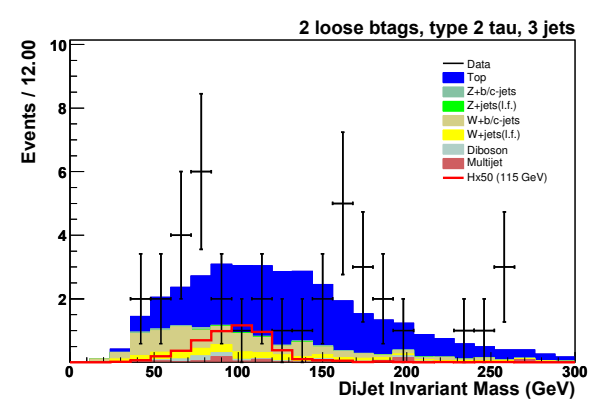

(b) dijet mass for 3 jet events

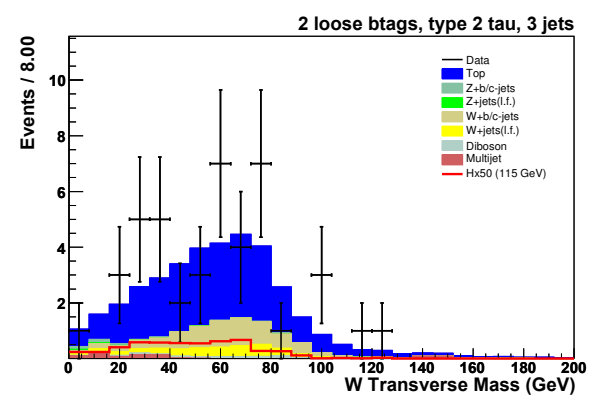

(d) $m_{\mathrm{T}}(W)$ for 3 jet events

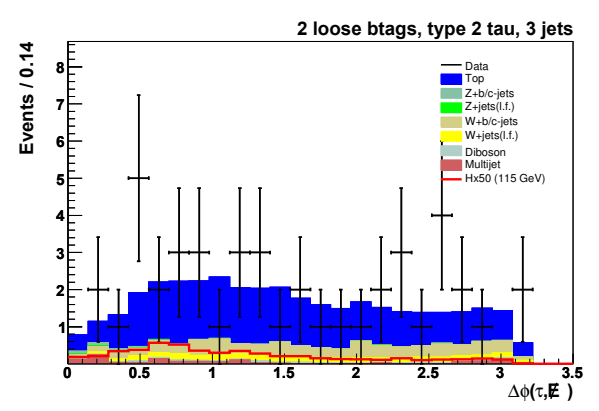

(f) $\Delta \phi\left(\tau, \mathbb{E}_{\mathrm{T}}\right)$ for 3 jet events

Figure 4.17: data versus background comparisons for the selection of tau type 2 events with two loose $b$-tags. 


\section{Chapter 5}

\section{Testing the Higgs Hypothesis}

In Chapter 4 different samples were selected to investigate whether there is a Standard-Model Higgs boson. The samples were split by $b$-tag operating point, tau type and jet multiplicity, yielding eight independent channels where measured data can be compared to signal and background models. These models consist of Monte-Carlo simulations for signal and most of the backgrounds. A sideband region is used to assess the multijet background.

In this chapter, it is discussed how the discriminatory power is maximized for each of the channels. First, we strive for maximal separation between signal and background, using Boosted Decision Trees (BDTs). Then we will discuss the uncertainty on our estimates, both statistical and systematic, followed by the hypothesis testing. The hypothesis that a SM Higgs boson exists is not confirmed, so we conclude with setting limits on its cross section.

Because the signal contribution depends on the Higgs mass, the hypothesis that a Higgs boson exists is divided into different hypotheses; we take $m_{H}=$ 100 to $150 \mathrm{GeV}$, with $5 \mathrm{GeV}$ intervals as test masses. Although the selection procedure was independent of the Higgs mass, we treat the Higgs hypothesis for each tested $m_{H}$ differently starting with the BDT training. This ensures that the information that is present in the events is used optimally to distinguish signal from background.

As in Chapter 4, this chapter is an expansion of the description in [76], which is based on earlier work in [77, 78.

\subsection{Boosted Decision Trees}

It is useful to combine the information of measured events across variables; correlations between variables may provide additional handles to separate signal and 
background. For two variables, extracting this information is relatively straightforward, but to do this for many variables is harder. For a large number of variables, the analysis can easily be limited by the available statistics due to enormous combinatorics; if there are $n$ variables, even making a simple binary distinction in each variable results in $2^{n}$ categories. Since we use a total of 15 variables, the number of categories would become 32768 , a number impossible to deal with in practice.

Techniques that deal with the problem of extracting most information from a multivariate environment are called multivariate analyses. They are trained on samples where it is known which event is signal, and which is background. The training is meant to find regions in the $n$-dimensional variable space where signal and background are distributed differently, hence can be distinguished. The result is a single output variable, which is the combination of input variables that best describes the overall signal similarity of an event.

The multivariate analysis is trained on one third of each MC-generated set listed in Tables 4.44 .5 and 4.6. It is tested for overtraining on another third. The remaining events are unbiased and are used to estimate the signal and background of the output in order to compare them to measured data.

Some MC sets have fewer than 300 events. For those sets, training against them turns out to be less useful. Furthermore, splitting them in a training, testing and unbiased subset will increase the statistical uncertainties of the final estimates. Therefore, they are not split and trained or tested on; they are only used as unbiased estimator for the trained multivariate analysis. Also the multijet background is not used for training or testing.

\subsubsection{Introduction to Boosted Decision Trees}

For the $W H \rightarrow \tau \nu b \bar{b}$ analysis, we use Boosted Decision Trees [91] as a multivariate technique. These consist of a weighted combination of several single decision trees.

An example of a decision tree is depicted in Figure 5.1 a sample is divided into two subsamples by placing a simple cut on a chosen variable. These subsamples are independently split into smaller subsamples using cuts on a new variable. This procedure continues iteratively until signal-like or background-like subsamples (leafs) remain. This 'training' of the tree is explained more in Section5.1.2.

Next, the decision tree is 'boosted' by giving higher weights to events in leafs with a bad separation. After boosting, a new tree is trained using the altered weights. The boosting procedure is described further in Section5.1.3. When a certain number of boosts is performed, the trees are added with relative weights. This forms the final trained BDT.

A Boosted Decision Tree is trained for every channel. Because the signal 


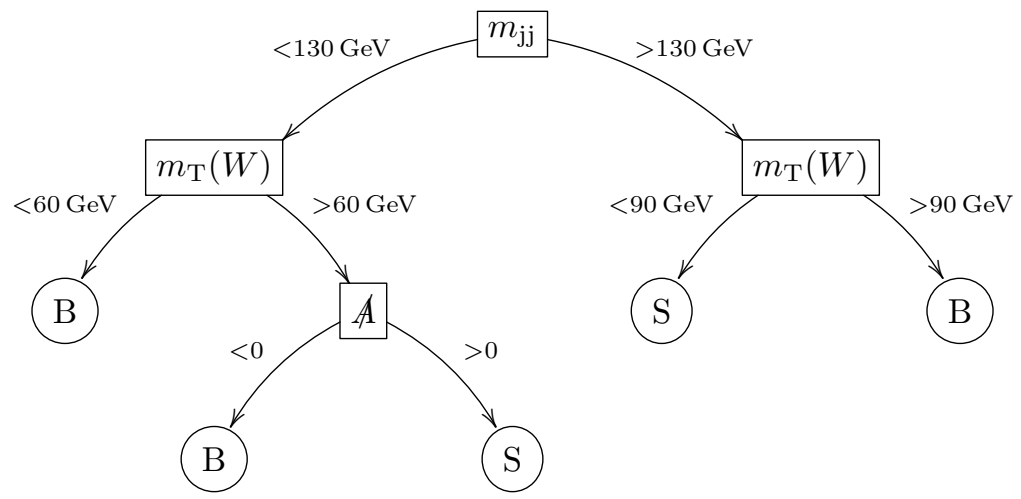

Figure 5.1: schematic example of a decision tree. The circles represent signal or background leafs, while the boxes are the decision nodes.

signature depends on the Higgs mass, every channel needs a separate BDT for each value of $m_{H}$. This results in a total of 88 BDTs.

\subsubsection{Decision Tree Training}

At first, the MC events have weights due to their cross sections and the various reweightings. These weights are called $w_{i}$ for event $i$ and will be altered in the boosting process discussed later. Before training and boosting start, the events are reweighted so that the sum of all signal weights equals the sum of all background weights. This ensures that the BDT training will give a maximal separation.

The splitting of a sample into two subsamples takes place at a 'node'. Such a node uses several training variables. First, the purity $P$ is defined as the weighted signal fraction of events:

$$
P \equiv \frac{\sum_{\text {signal }} w_{i}}{\sum_{\text {all }} w_{i}},
$$

where $w_{i}$ represents the weight of event $i$. Also the Gini index

$$
G \equiv P(1-P) \sum_{\text {all }} w_{i}
$$

is calculated. The Gini index is a measure of similarity, so a lower value indicates more separation.

Then, a cut is placed on one of the variables. Both the variable and the cut position are chosen such that the sum of the Gini-indices of the two resulting new nodes is minimized (one with events that did pass, and one with the events 
that did not pass the cut). This ensures maximal separation into signal-like and background-like subsamples.

When the node has fewer than 100 events, the splitting stops and the node is called a leaf. It is classified as a signal or background leaf according to the purity: $P>0.5$ for signal, $P<0.5$ for background.

\subsubsection{Boosting}

We use a boosting method called AdaBoost [92].

An event gains weight if it is misclassified, to make the next tree try harder to identify it correctly. To do this, we first define the error of a trained tree $j$ :

$$
E^{j} \equiv \frac{\sum_{i} w_{i}^{j} M_{i}^{j}}{\sum_{i} w_{i}^{j}},
$$

where $M_{i}^{j}=1$ if the $i^{\text {th }}$ event was misclassified in the tree, and $M_{i}^{j}=0$ otherwise. Then, the new event weight is given by

$$
w_{i}^{j+1} \equiv w_{i}^{j} \exp \left(\alpha^{j} M_{i}^{j}\right)
$$

where $\alpha^{j}$ is the overall weight of the tree, which is calculated by

$$
\alpha^{j} \equiv \beta \ln \left(\frac{1-E^{j}}{E^{j}}\right) .
$$

This analysis uses $\beta=0.18$, a value adopted from other $\mathrm{D} \varnothing$ analyses [80]. Note that since the classification outperforms random guessing, $E^{j}$ will be smaller than $1 / 2$, so $\alpha^{j}$ is always positive. This means that events that are harder to classify gain weight, making it more important for the next decision tree to improve its performance on them.

The boosting procedure is repeated 20 times, or if the testing method described below detects saturation.

\subsubsection{Testing}

The trees are tested using the MC test samples to prevent overtraining.

The events in the unbiased samples, which are used for the hypothesis testing, get a BDT output value by

$$
\text { BDT output }_{i} \equiv \sum_{j} \alpha^{j} P_{i}^{j}
$$


where $P_{i}^{j}$ is the purity of the leaf in tree $j$ where event $i$ ended. This output value is also calculated for the testing samples. If the performance ${ }^{1}$ of the BDT no longer improves on the testing samples, further boosting is stopped.

\subsubsection{BDT Input Variables}

The variables used in the BDT for the analysis in this thesis are listed in Table 5.1 . together with their relative importance. This importance is defined by the difference in Gini index a variable introduces in all nodes $k$ of every tree $j$, weighted by the $\alpha^{j}$ of each tree:

$$
\text { relative importance }=\sum_{j, k} \alpha^{j} \Delta G_{k}^{j},
$$

with

$$
\Delta G=G_{\text {parent node }}-G_{\text {left child }}-G_{\text {right child }} .
$$

Because a lower Gini index signifies a better separation, a higher value for $\Delta G$ indicates more discriminating power in the BDT.

The relative importance of the input variables gives a flavor of the relevance of these variables in distinguishing signal from background. It is used as a sanity check (the dijet invariant mass $m_{\mathrm{jj}}$ should for example be important), but not used in any further calculation.

\subsubsection{BDT Output Distributions}

The trained BDT are applied to the unbiased signal and background MC samples, and on data. The resulting output, calculated with Equation (5.6), is plotted in Figure 5.2 for the two channels with the best $s / \sqrt{b}$, at different Higgs mass hypotheses. The 2-b-tag sample generally shows a better separation than the 1-tag sample. Figure 5.3 depicts the other six channels at $m_{H}=115 \mathrm{GeV}$.

\subsection{Uncertainties}

Now we have the signal and background estimates in each bin of the BDT outputs. They are given by the sum of the weights $w$ of the MC-generated and QCD events in that bin:

$$
N_{H}=\sum_{i} w_{i}
$$

\footnotetext{
${ }^{1}$ This can be defined by the sum over the BDT output with -1 weight for background events and +1 for signal events.
} 


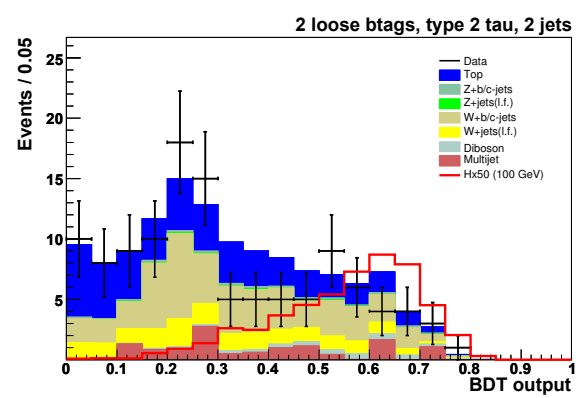

(a) BDT output $2 b$-tag, for $m_{H}=100 \mathrm{GeV}$

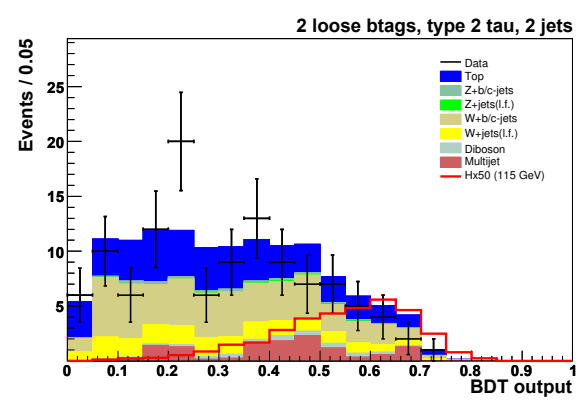

(c) BDT output $2 b$-tag, for $m_{H}=115 \mathrm{GeV}$

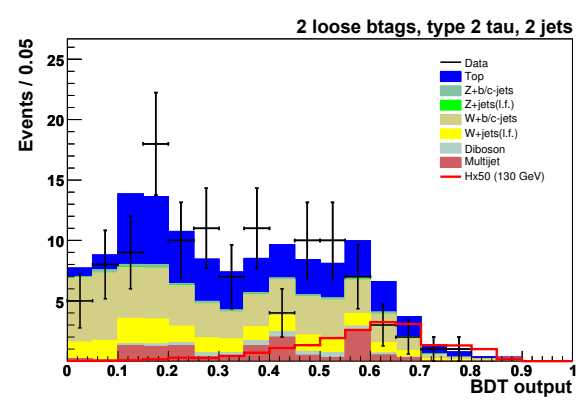

(e) BDT output $2 b$-tag, for $m_{H}=130 \mathrm{GeV}$

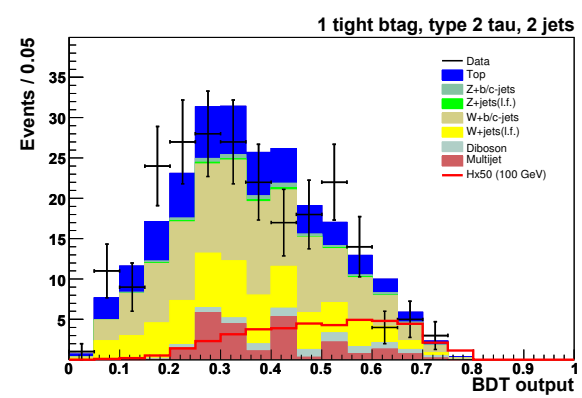

(b) BDT output $1 b$-tag, for $m_{H}=100 \mathrm{GeV}$

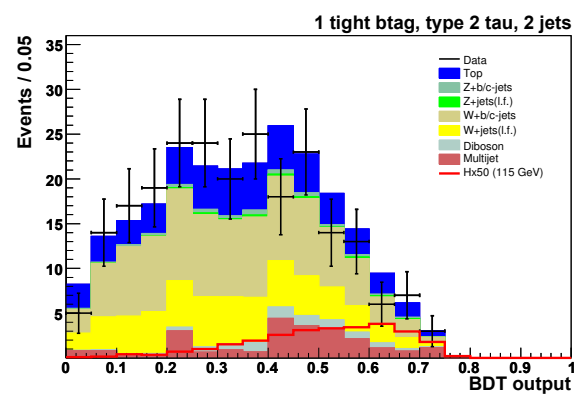

(d) BDT output $1 b$-tag, for $m_{H}=115 \mathrm{GeV}$

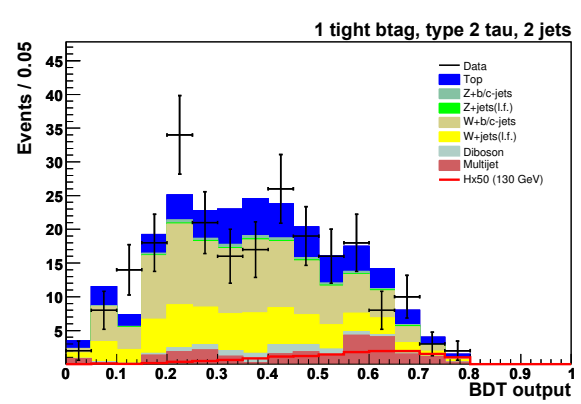

(f) BDT output $1 b$-tag, for $m_{H}=130 \mathrm{GeV}$

Figure 5.2: BDT outputs for the tau type 2 samples with 2 jets, for 2 loose or 1 tight $b$-tagged events and different Higgs masses. The signal contribution is multiplied by a factor 50 , to show its shape. 


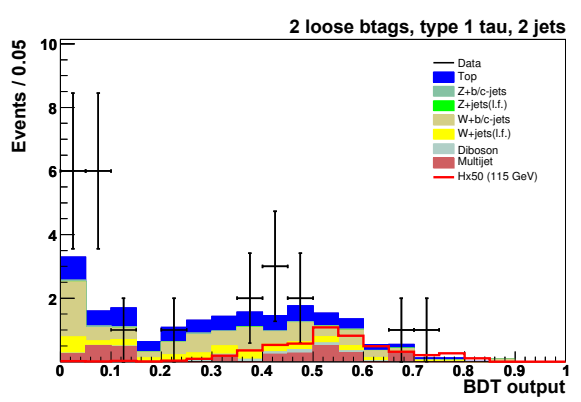

(a) BDT output 2 b-tag, tau type 1, 2 jets

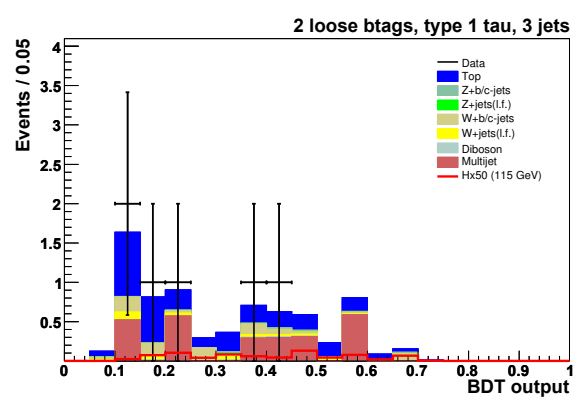

(c) BDT output 2 b-tag, tau type 1,3 jets

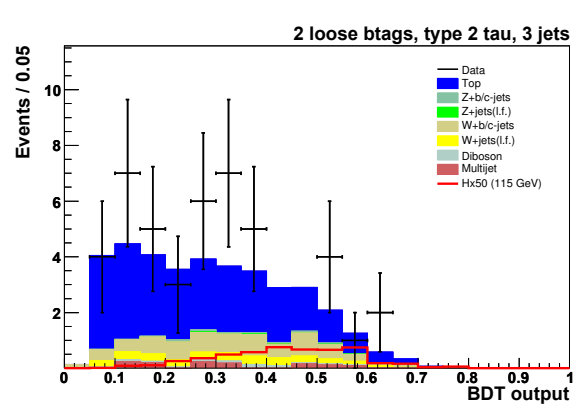

(e) BDT output 2 b-tag, tau type 2, 3 jets

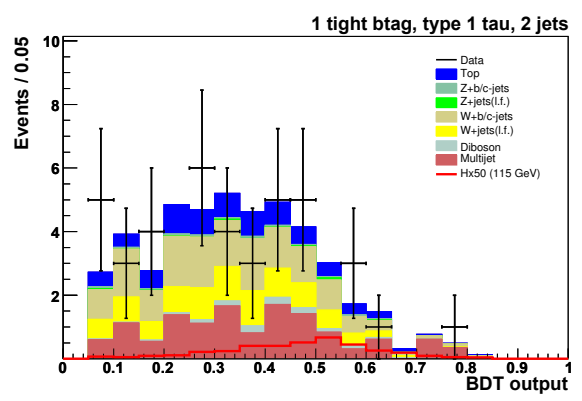

(b) BDT output $1 b$-tag, tau type 1,2 jets

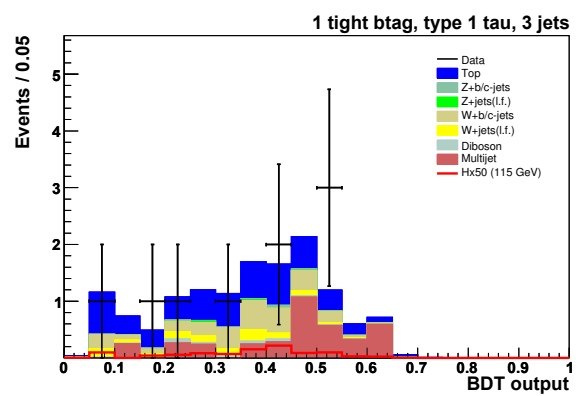

(d) BDT output $1 b$-tag, tau type 1,3 jets

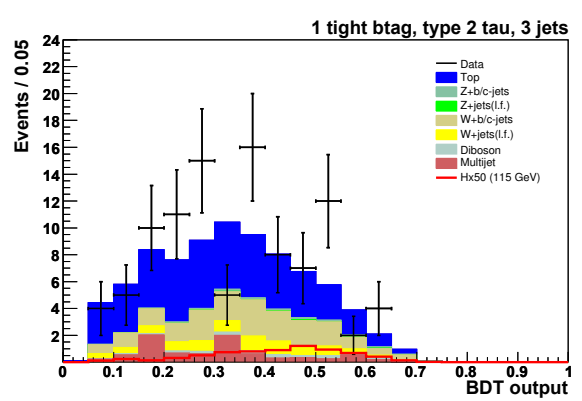

(f) BDT output $1 b$-tag, tau type 2,3 jets

Figure 5.3: BDT outputs at $m_{H}=115 \mathrm{GeV}$ for the statistically less-significant samples. The signal contribution is multiplied by a factor 50 , to show its shape. 


\begin{tabular}{|c|c|c|c|c|c|c|c|c|}
\hline \multirow[b]{3}{*}{ Variable } & \multicolumn{4}{|c|}{ OneTight $b$-tag } & \multicolumn{4}{|c|}{ TwoLoose $b$-tag } \\
\hline & \multicolumn{2}{|c|}{ Type 2} & \multicolumn{2}{|c|}{ Type 1} & \multicolumn{2}{|c|}{ Type 2} & \multicolumn{2}{|c|}{ Type 1} \\
\hline & 2 jets & 3 jets & 2 jets & 3 jets & 2 jets & 3 jets & 2 jets & 3 jets \\
\hline$m_{\mathrm{jj}}$ & 19.04 & 8.74 & 33.10 & 14.85 & 45.59 & 31.07 & 51.64 & 44.91 \\
\hline$\Delta \phi\left(\tau, \mathbb{E}_{\mathrm{T}}\right)$ & 6.60 & 6.98 & 5.30 & 10.99 & 7.01 & 8.98 & 6.53 & 10.66 \\
\hline A & 4.74 & 6.88 & 4.34 & 5.76 & 5.74 & 12.28 & 2.20 & 5.85 \\
\hline$H_{\mathrm{T}}$ & 6.42 & 3.35 & 5.68 & 1.99 & 3.23 & 6.10 & 1.27 & 0.00 \\
\hline$H_{\mathrm{T}}($ jets $+\tau)$ & 2.25 & 3.21 & 8.15 & 1.25 & 3.62 & 4.64 & 5.43 & 1.39 \\
\hline$p_{\mathrm{T}}($ jet 2$)$ & 3.39 & 6.02 & 8.82 & 4.76 & 4.28 & 5.60 & 4.78 & 5.96 \\
\hline$p_{\mathrm{T}}^{\mathrm{trk}}$ & 4.40 & 6.42 & 6.30 & 3.96 & 4.91 & 8.79 & 2.90 & 0.00 \\
\hline$\Delta \phi($ jet 1, jet 2$)$ & 3.81 & 4.06 & 6.53 & 4.49 & 4.99 & 9.94 & 5.49 & 4.50 \\
\hline$m_{\mathrm{T}}(W)$ & 4.21 & 6.00 & 4.74 & 0.77 & 6.33 & 10.21 & 5.33 & 8.95 \\
\hline$p_{\mathrm{T}}($ jet 1$)$ & 2.92 & 4.22 & 5.79 & 4.20 & 3.53 & 5.35 & 3.66 & 7.01 \\
\hline$\eta($ jet 2$)$ & 4.29 & 4.54 & 4.28 & 6.63 & 3.46 & 6.60 & 2.80 & 9.89 \\
\hline$\eta($ jet1) & 3.47 & 4.87 & 3.83 & 4.24 & 4.46 & 6.52 & 3.41 & 1.71 \\
\hline$p_{\mathrm{T}}(\tau)$ & 2.23 & 3.45 & 3.87 & 6.51 & 3.14 & 6.42 & 4.25 & 3.52 \\
\hline$\not H_{\mathrm{T}}($ jets $+\tau)$ & 2.50 & 3.56 & 5.08 & 3.34 & 3.90 & 4.79 & 4.29 & 1.78 \\
\hline $\mathbb{E}_{\mathrm{T}}$ & 2.40 & 1.40 & 2.23 & 3.70 & 3.25 & 3.81 & 1.87 & 5.33 \\
\hline
\end{tabular}

Table 5.1: input variables to the BDT and their relative importance as defined in Equation (5.7) for the case $m_{H}=115 \mathrm{GeV}$. The variables $H_{\mathrm{T}}$ and $p_{\mathrm{T}}^{\text {trk }}$ in the $2 b$-tag, tau type 1,3 -jet channel give a zero contribution. This is due to the limited statistics in this channel, which causes the training and boosting to stop before these variables were used.

where $i$ runs over every event in hypothesis $H$, which can be the signal and background $(\mathrm{S}+\mathrm{B})$ or background only $(\mathrm{B})$ hypothesis.

Because the number of events used for the estimation is finite, there is a statistical uncertainty given by

$$
\sigma_{\text {stat }}\left(N_{H}\right)=\sqrt{\sum_{i} w_{i}^{2}} .
$$

This statistical uncertainty reflects our lack of knowledge of what we would measure on average if we would perform the measurement infinitely many times. It is not to be confused with the Poisson uncertainty, which is a measure for the spread of a single measurement, and is often quoted as $\sqrt{N_{H}}$.

On top of the statistical uncertainties there are systematic uncertainties, arising from unknown biases introduced by the method of the analysis. There are 
various sources of systematic uncertainty and they can affect signal and background estimates.

\subsubsection{Flat Systematics}

Some systematic uncertainties are directly proportional to the background they affect. For a flat systematic $u$, the uncertainty on the background estimate $b$ is given by

$$
\sigma_{u}\left(N_{b}\right)=A_{u} N_{b}
$$

where $A_{u}$ is a constant of the flat systematic uncertainty and $N_{b}$ is calculated as in Equation (5.9), but with the sum running over the affected background(s) only. Because different background contributions have different shapes in the BDT output, the 'flat' systematics do not give rise to a flat uncertainty on the background estimate as a whole.

Uncertainties on the signal contribution are treated similarly.

The following systematic uncertainties give rise to a flat systematic:

- The tau-identification uncertainty was calculated in a $Z \rightarrow \tau \tau$ sample and includes the uncertainty on the $\mathrm{NN}_{\tau}$ and tau track. It is $11 \%$ for tau type 1 events and $4.5 \%$ for type 2 taus [73];

- Uncertainties on taggability and vertex confirmation scale factors are respectively $2 \%$ and $5 \%$, as reported by the $Z H \rightarrow \nu \nu b \bar{b}$ analysis [80];

- The uncertainty on the multijet background comes from the reweightings described in Section 4.3.4. The amplitude of the uncertainty is defined as the difference that the reweighting brought about in the integral number of events after all following cuts $\left(\mathbb{E}_{\mathrm{T}}^{\mathrm{SIG}}>4.5, \Delta \phi\left(\not p_{\mathrm{T}}^{\mathrm{trk}}, \mathbb{E}_{\mathrm{T}}\right)<2\right.$ and $b$ tagging). The uncertainty is found to be $12 \%$ for type 1 tau events and $7 \%$ for type 2 tau events;

- The uncertainty on the Higgs cross section is $6 \%$ and is taken from the Tevatron New Phenomena \& Higgs Working Group [93];

- We have taken the diboson cross-section uncertainty of $7 \%$ from NLO QCD computations and parton-shower simulations [94;

- The uncertainty on the single-top cross section is $10 \%$, as quoted by the paper on single-top production at the Tevatron [95];

- For the top cross section, the uncertainty is $10 \%$ too. It is determined using heavy quark pair production at two loops in QCD [96];

- As recommended by the Higgs group, we have taken the uncertainty on the $W / Z+$ jets cross section to be $6 \%$ [97; 
- A heavy-flavor scale factor is applied to set the $W / Z+$ h.f. contributions relative to $W / Z+$ l.f. ones. According to MCFM, the total uncertainty on the cross section of $W / Z+b / c$-jets is $+25 /-19 \%$ [98. However, this is composed of the light-flavor uncertainty and the exclusive heavy-flavor scale-factor uncertainty. We split this into the $6 \%$ uncertainty on the $W / Z+$ jets cross section that was mentioned before, and a $20 \%$ uncertainty on the heavyflavor factor. This is done to ensure the $W / Z+$ jets part of the uncertainty on the $W / Z+$ h.f. sample is fully correlated with the $W / Z+$ jets uncertainty on the $W / Z+$ l.f. sample;

- The integrated-luminosity uncertainty is $6.1 \%$ [99];

- The trigger parameterization has a $5 \%$ uncertainty, taken from the $Z H \rightarrow$ $\nu \nu b \bar{b}$ study [80. An additional $2 \%$ uncertainty is added in quadrature to account for the assumption that the tau turn-on is the same as the jet turnon in the parameterization upgrade used for this analysis [78. This yields a total uncertainty of $5.5 \%$ on this quantity;

- For the Parton Distribution Function (PDF) determination, 20 parameters are fit to Tevatron jet cross-section measurements [100]. After the fit, the parameters have a residual uncertainty defined by the values where they would increase the $\chi^{2}$ of the fit with a certain maximum amount. These uncertainties in the fit parameters also introduce uncertainties on the signal and background estimates. For all 20 uncertainties, their contribution to these estimates is determined. The maximum of these contributions is taken as the overall PDF uncertainty on the estimates. Based on the studies undertaken by the $Z H \rightarrow \nu \nu b \bar{b}$ analysis [80, we take $2 \%$ and $3 \%$ for signal and background uncertainties, respectively. We take the signal and background PDF uncertainties to be fully correlated;

- The amount of uncertainty coming from jet-reconstruction and identification is attained by the impact of a $\pm 1 \sigma$ fluctuation of jet reconstruction and ID uncertainties quoted by [56]. They are flat in the BDT output, but depend on the number of $b$-tags and the tau type. Their values are listed in Table 5.2 .

\subsubsection{Shape Systematics}

Some systematic uncertainties have values that depend on the BDT output, so the systematic will no longer have a constant $A_{u}$ of Equation (5.11). These systematics are determined by running the analysis again, but this time with the scenarios of $\pm 1 \sigma$ fluctuations. The relative difference with the nominal scenario has a shape in the BDT-output distributions, which can be taken as a measure of the uncertainty due to this shape systematic. Effectively, the shape means a 


\begin{tabular}{lrc}
\hline Sample & Signal MC & Background MC \\
\hline OneTight $b$-tag, tau type 1 events & $2.4 \%$ & $1.5 \%$ \\
OneTight $b$-tag, tau type 2 events & $1.8 \%$ & $0.8 \%$ \\
TwoLoose $b$-tag, tau type 1 events & $3.2 \%$ & $2.4 \%$ \\
TwoLoose $b$-tag, tau type 2 events & $2.3 \%$ & $2.1 \%$ \\
\hline
\end{tabular}

Table 5.2: $A_{u}$ values of the jet-reconstruction and identification uncertainty for different samples.

different uncertainty amplitude is present for every bin of the BDT output. We define in every bin

$$
A_{b}^{u \pm} \equiv \frac{N_{b}^{ \pm \sigma}-N_{b}^{\text {nominal }}}{N_{b}^{\text {nominal }}}
$$

for background (or signal) contribution $b$ and the $\pm 1 \sigma$ scenario of uncertainty $u$.

The following shape systematics are taken into account.

- The effect of ALPGEN MLM and scale uncertainties ${ }^{2}$ on the input variables of the BDT are determined in [101, 102]. For example $m_{\mathrm{jj}}$ changes in the order of $1 \%$ by $\pm 1 \sigma$ variations. The changes are propagated to the BDT outputs, where the shape of the uncertainties is determined;

- The JES and JER systematic uncertainties are done by varying the jet shifting and smearing done by JSSR by the $\pm 1 \sigma$ uncertainties quoted in [56];

- Effects of $b$-tagging efficiency uncertainties are also taken as a shape systematic. These uncertainties are taken from [67.

The relative uncertainties that the shape systematics introduce are shown in Figure 5.4 for the samples with the best $s / \sqrt{b}$ ratio: the tau type 2, 2 jets samples with 1 tight or 2 loose $b$-tags.

As a summary, all systematic uncertainties for this $W H \rightarrow \tau \nu b \bar{b}$ analysis are listed in Table 5.3. A flat systematic is listed with its $A_{u}$ value, while the effects of shape systematics depend on the BDT output of the event.

\footnotetext{
${ }^{2}$ These uncertainties are related to the inner workings of the ALPGEN event generator. The MLM uncertainty arises from choices in the MLM parton-matching algorithm which protects the generator from double counting partons (the minimum $p_{\mathrm{T}}$ of a parton to be considered in the matching is such a choice). The scale uncertainty includes for example the uncertainty resulting from the choice at which scale the strong-coupling constant is evaluated at every vertex.
} 


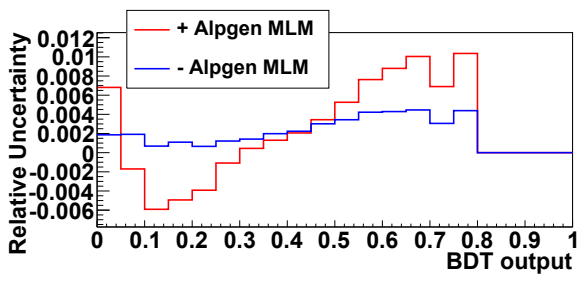

(a) Alpgen MLM uncertainty in the $2 b$-tag sample

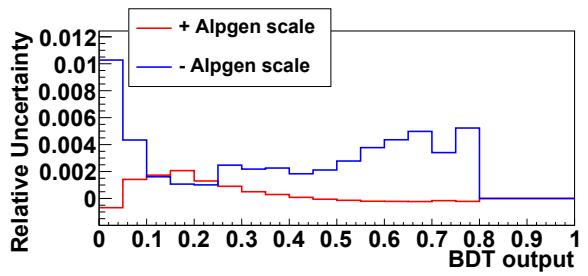

(c) Alpgen scale uncertainty in the 2 b-tag sample

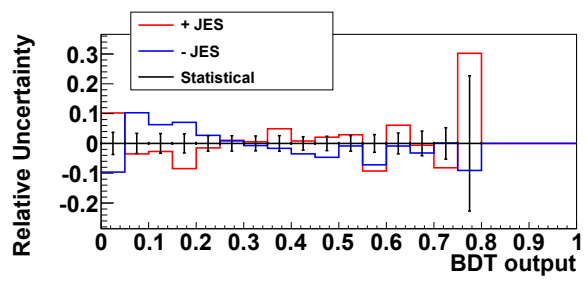

(e) JES uncertainty in the $2 b$-tag sample

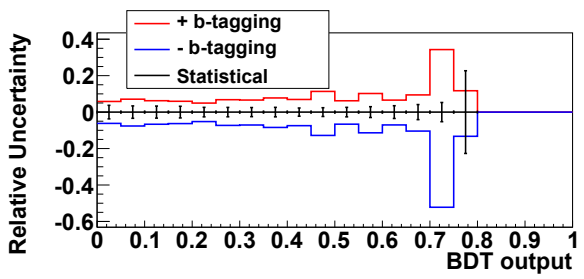

(g) $b$-tagging uncertainty in the $2 b$-tag sample

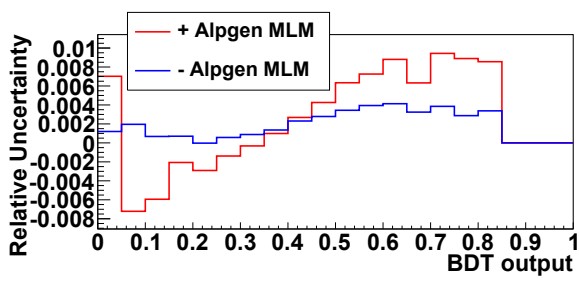

(b) Alpgen MLM uncertainty in the $1 b$-tag sample

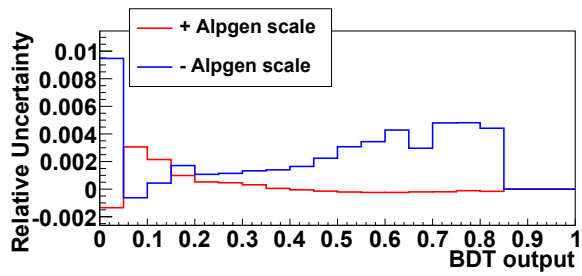

(d) Alpgen scale uncertainty in the $1 b$-tag sample

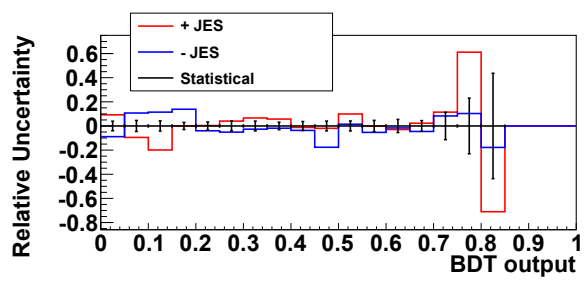

(f) JES uncertainty in the $1 b$-tag sample

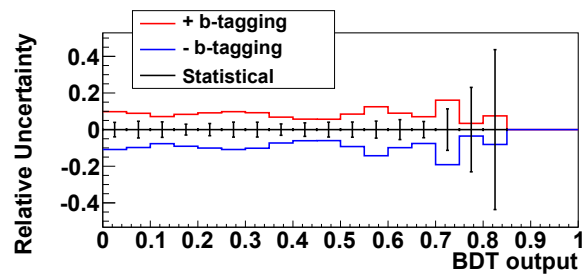

(h) b-tagging uncertainty in the $1 b$-tag sample

Figure 5.4: relative uncertainties on the background estimate for BDT outputs of tau type 2 samples with 2 jets, for 2 loose or 1 tight $b$-tagged events at $m_{H}=115 \mathrm{GeV}$. On the graphs of the JES and $b$-tagging uncertainties, the relative statistical uncertainty is also shown. 


\begin{tabular}{|c|c|c|c|}
\hline Systematic & $A_{\mathrm{u}}(\%)$ & Affects samples & Reference \\
\hline Tau ID for type 1 (2) & $11(4.5)$ & All MC & 73 \\
\hline QCD for tau type $1(2)$ & $12(7)$ & QCD sample & Section 4.3 .4 \\
\hline Higgs cross section & 6 & Signal MC & [93 \\
\hline Diboson cross section & 7 & Diboson MC & 94 \\
\hline Single Top cross section & 10 & Single Top MC & 95 \\
\hline Top cross section & 10 & Top MC & 96 \\
\hline $\mathrm{W} / \mathrm{Z}+$ jets cross section & 6 & $W / Z+$ jets $\mathrm{MC}$ & 97 \\
\hline Heavy Flavor Ratio & 20 & $W+b / c-$ jets $\mathrm{MC}$ & 98 \\
\hline Luminosity & 6.1 & All MC & 99 \\
\hline Trigger & 5.5 & All MC & 79 \\
\hline Taggability & 2 & All MC & 80 \\
\hline Vertex Confirmation SF & 5 & All MC & 80 \\
\hline Signal PDF & 2 & Signal MC & 80, 100 \\
\hline Background PDF & 3 & Background MC & 80,100 \\
\hline Jet reconstruction and ID & see Table 5.2 & All MC & 56 \\
\hline Jet Energy Scale & Shape & All MC & 56 \\
\hline Jet Energy Resolution & Shape & All MC & 56 \\
\hline BTag Uncertainty & Shape & All MC & 67 \\
\hline Alpgen MLM Uncertainty & Shape & $W / Z+$ l.f. $\mathrm{MC}$ & 101, 102 \\
\hline Alpgen Scale uncertainty & Shape & $W / Z+$ jets $\mathrm{MC}$ & 101, 102 \\
\hline
\end{tabular}

Table 5.3: sources, values and affected samples of the systematic uncertainties.

\subsection{Hypothesis Testing}

The following step is to compare the hypothesis that a SM Higgs boson with mass $m_{H}$ exists, with the hypothesis that there is no such boson. We use a semifrequentist approach [103, 104, which will be explained further in this section. In Section 5.4 the results of the $W H \rightarrow \tau \nu b \bar{b}$ analysis will be presented.

\subsubsection{Negative Log Likelihood Ratio}

We need a single parameter that signifies the compatibility of the measured data to both hypotheses. To do this, we first introduce the likelihood ratio $Q$. It is defined as

$$
Q \equiv \frac{L(\mathrm{~S}+\mathrm{B} \mid \text { data })}{L(\mathrm{~B} \mid \text { data })}
$$

where $L(H \mid$ data $)$ is the likelihood for hypothesis $H$, given the data, which is defined as the probability that the data are measured, if the hypothesis is true. 
The probability of measuring $d$ events in a bin where $h$ events are expected, is given by the Poisson distribution:

$$
p(d \mid b)=\frac{e^{-h} h^{d}}{d !} .
$$

Therefore, in case of a single bin $i$, Equation 5.13 becomes

$$
Q_{i}=\frac{L\left(s_{i}+b_{i} \mid d_{i}\right)}{L\left(b_{i} \mid d_{i}\right)}=\frac{p\left(d_{i} \mid s_{i}+b_{i}\right)}{p\left(d_{i} \mid b_{i}\right)}=e^{-s_{i}}\left(\frac{s_{i}+b_{i}}{b_{i}}\right)^{d_{i}},
$$

where $s_{i}$ and $b_{i}$ are the expected amount of signal and background in the bin.

For all bins in all independent channels, the total likelihood ratio is given by

$$
Q=\frac{p(\text { data } \mid \mathrm{S}+\mathrm{B})}{p(\text { data } \mid \mathrm{B})}=\frac{\prod_{i} p\left(d_{i} \mid s_{i}+b_{i}\right)}{\prod_{i} p\left(d_{i} \mid b_{i}\right)}=\prod_{i} Q_{i} .
$$

Because of computational efficiency, the negative log likelihood ratio, also called Log Likelihood Ratio (LLR), is used. It is defined as

$$
\begin{aligned}
\mathrm{LLR} & \equiv-2 \ln (Q) \\
& =-2 \sum_{i} \ln Q_{i} \\
& =2 \sum_{i}\left[s_{i}-d_{i} \ln \left(\frac{s_{i}+b_{i}}{b_{i}}\right)\right] .
\end{aligned}
$$

The LLR is a convenient separator between the background and signal hypotheses; it will not lose sensitivity if more measurements are included, either by increasing the statistics or by including additional channels or bins.

\subsubsection{Nuisance Parameters}

Unfortunately, the signal and background hypotheses do not give infinitely precise predictions on $s_{i}$ and $b_{i}$; as explained in Section 5.2, there are systematic uncertainties that have to be taken into account. They are incorporated as so-called nuisance parameters, which are denoted as $\theta_{u}$ for an uncertainty $u$. Together, all uncertainties form a vector $\vec{\theta}$, which has the dimension of the number of systematic uncertainties. So the signal and background estimates in bin $i$ become $s_{i}(\vec{\theta})$ and $b_{i}(\vec{\theta})$. For a hypothesis with nominal estimate $h_{i}^{\text {nom }}$ in bin $i$, the estimate given nuisance parameters $\vec{\theta}$ is written as

$$
h_{i}(\vec{\theta})=h_{i}^{\mathrm{nom}} \prod_{u}\left(1+A_{u, i} S_{u}\right) .
$$


$A_{u, i}$ is the standard deviation of nuisance parameter $u$ in the bin as defined in Equation (5.11). $S_{u}$ represents the deviation from the central value of the nuisance parameter $\theta_{u}$; it is gaussian distributed with mean value 0 and standard deviation 1.

The systematic uncertainties are generally estimated (and usually overestimated, for the sake of caution). ${ }^{3}$ Therefore it is useful to determine the true value of $\vec{\theta}$ as well as possible. From this we get a better estimate of the true $s_{i}$ and $b_{i}$ and reduce the smearing caused by the nuisance parameters.

We fit the true value of $\vec{\theta}$ by minimizing

$$
\chi^{2}(H, \vec{\theta}, d)=-2 \ln (p(d, \vec{\theta} \mid H)),
$$

where $p(d, \vec{\theta} \mid H)$ is the probability that the nuisance parameter had true value $\vec{\theta}$ and the measured data was $d$ if the hypothesis $H$ is true. Using $p(A, B)=$ $p(A \mid B) p(B)$, we get

$$
\begin{aligned}
\chi^{2}(H, \vec{\theta}, d) & =-2 \ln (p(d \mid \vec{\theta}, H) p(\vec{\theta} \mid H)) \\
& =-2 \ln \left(\prod_{i} \frac{e^{-h_{i}} h_{i}^{d_{i}}}{d_{i} !}\right)-2 \ln \left(\prod_{u} \frac{e^{-\frac{1}{2} S_{u}^{2}}}{\sqrt{2 \pi}}\right) \\
& =2 \sum_{i}\left[h_{i}-d_{i} \ln \left(h_{i}\right)+\ln \left(d_{i} !\right)\right]+\sum_{u}\left(S_{u}^{2}+\ln 2 \pi\right),
\end{aligned}
$$

where we used the Poisson distribution of $p(d \mid \vec{\theta}, H)$ and the gaussian distributed nature of $S_{u}$. To find the minimum of $\chi^{2}(\vec{\theta})$, we are only interested in the terms that depend on $\vec{\theta}$, so minimizing

$$
\chi^{2}(H, \vec{\theta}, d)=2 \sum_{i}\left[h_{i}(\vec{\theta})-d_{i} \ln \left(h_{i}(\vec{\theta})\right)\right]+\sum_{u} S_{u}^{2}
$$

suffices.

The corresponding LLR now becomes

$$
\begin{aligned}
\mathrm{LLR} & =-2 \ln \left(\frac{p\left(d, \hat{\theta}^{\mathrm{S}+\mathrm{B}} \mid \mathrm{S}+\mathrm{B}\right)}{p\left(d, \hat{\theta}^{\mathrm{B}} \mid \mathrm{B}\right)}\right) \\
& =\chi^{2}\left(\mathrm{~S}+\mathrm{B}, \hat{\theta}^{\mathrm{S}+\mathrm{B}}, d\right)-\chi^{2}\left(\mathrm{~B}, \hat{\theta}^{\mathrm{B}}, d\right) \\
& =2 \sum_{i}\left[\hat{h}_{i}^{\mathrm{S}+\mathrm{B}}-\hat{h}_{i}^{\mathrm{B}}-d_{i} \ln \left(\frac{\hat{h}_{i}^{\mathrm{S}+\mathrm{B}}}{\hat{h}_{i}^{\mathrm{B}}}\right)\right]+\sum_{u}\left[\left(\hat{S}_{u}^{\mathrm{S}+\mathrm{B}}\right)^{2}-\left(\hat{S}_{u}^{\mathrm{B}}\right)^{2}\right],
\end{aligned}
$$

\footnotetext{
${ }^{3}$ There is no deliberate overestimation, but when a systematic uncertainty is estimated, usually the extreme values of the fluctuation are taken as a standard deviation. On average, this will result in an overestimation of the uncertainties, but it is impossible to affiliate the overestimation to specific systematics. Because an overestimation is less detrimental than an underestimation, the current method is used nonetheless.
} 
where $\hat{h}^{H}, \hat{\theta}^{H}$ and $\hat{S}^{H}$ denote the values for $h, \theta$ and $S$ corresponding to the best fit given hypothesis $H$.

\subsubsection{Confidence Levels}

To test the validity of a hypothesis, its Confidence Level (CL) is defined as the probability to measure an LLR greater (or equal) than the actually measured LLR, given the hypothesis $H$ :

$$
\mathrm{C} L_{H} \equiv p(\operatorname{LLR} \geq \operatorname{LLR}(d) \mid H) .
$$

Therefore, we need to know the probability density function of the LLR. This is determined by performing pseudo-experiments; first we generate a set of nuisance parameters. Then a data count is generated in each bin, using the Poisson distribution given hypothesis $H$ and the nuisance parameters. Next, we fit the nuisance parameters for both hypotheses using Equation (5.21). Finally, we use Equation (5.22) to determine the LLR for this generated datapoint. By repeating this procedure many times for both hypotheses, we arrive at the prior predictive ensembles of the hypotheses.

In Figure 5.5 examples of the prior predictive ensembles for an $\mathrm{S}+\mathrm{B}$ and a $\mathrm{B}$ hypothesis can be seen.

\section{$\mathrm{CL}_{\mathrm{S}}$}

In a strict Frequentist interpretation, $\mathrm{CL}_{\mathrm{S}+\mathrm{B}}$ is the only variable we can use to reject the $\mathrm{S}+\mathrm{B}$ hypothesis. In this case, a high measured value of LLR (say 15 in Figure 5.5 would lead to a clear rejection of the signal hypothesis. Unfortunately, the data does not appear to come from the background hypothesis either. Because we mainly want to discriminate between the B and S $\mathrm{B}$ hypotheses, we introduce

$$
\mathrm{CL}_{\mathrm{S}} \equiv \frac{\mathrm{CL}_{\mathrm{S}+\mathrm{B}}}{\mathrm{CL}_{\mathrm{B}}}
$$

Although this quantity cannot be interpreted as a confidence level in the strict sense, it does take into account the mismodeling of the background hypothesis. $\mathrm{CL}_{\mathrm{B}} \leq 1$, so $\mathrm{CL}_{\mathrm{S}}$ is more conservative than $\mathrm{CL}_{\mathrm{S}+\mathrm{B}}$ in rejecting a signal. It is not used to do a possible confirmation of a Higgs hypothesis.

A discussion on the interpretation of $\mathrm{CL}_{\mathrm{S}}$ can be found in [105].

\subsubsection{Limits on the Cross Section}

We declare a rejection of the $\mathrm{S}+\mathrm{B}$ hypothesis if the confidence level of rejection $\left(1-\mathrm{CL}_{\mathrm{S}}\right)$ is greater than $95 \%$. 


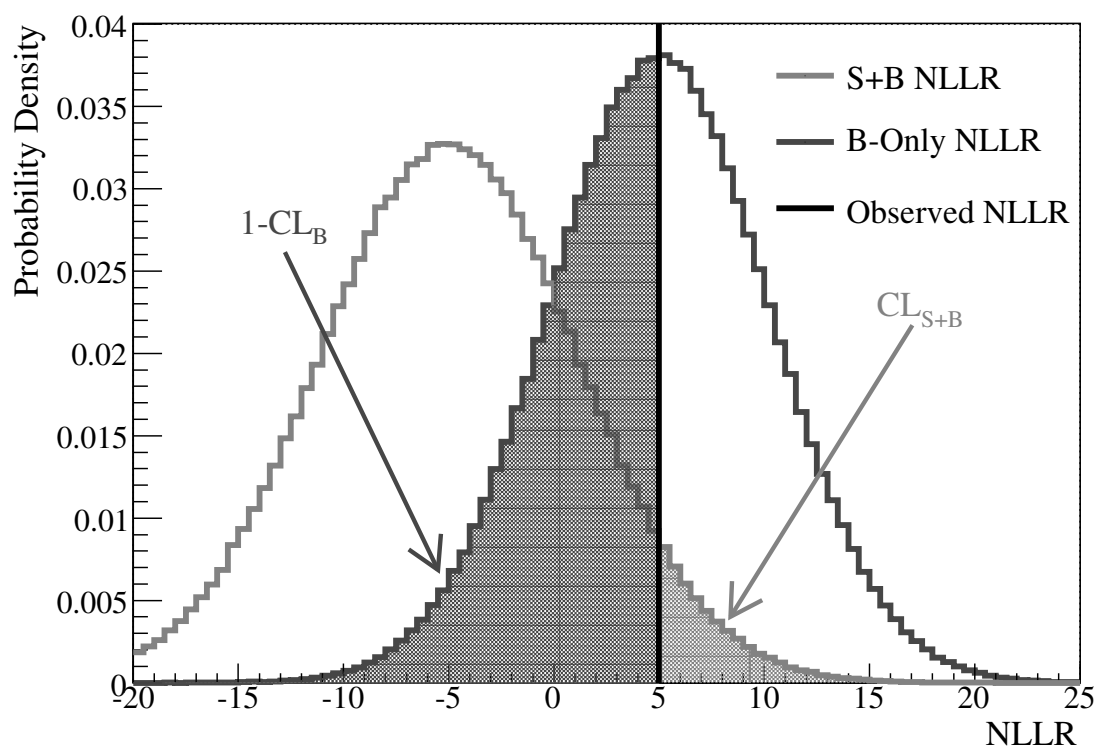

Figure 5.5: an example of the prior predictive ensembles of an $\mathrm{S}+\mathrm{B}$ and a $\mathrm{B}$ hypothesis. Also the Confidence Levels for the background and the signal hypotheses are shown. In this case the observed LLR coincides with the mean expected LLR for the background and has a $\mathrm{CL}_{\mathrm{S}}$ of $90 \%$.

Even if this confidence level is not achieved (and no significant signal has been observed), we can set a limit on the cross section of Higgs-boson production. Although the SM Higgs cross section is completely determined for a given $m_{H}$, we can artificially inflate it to the point where we would have been able to exclude the hypothesis. This is a useful way to determine the sensitivity of an analysis. It also allows to quantify when a particle from another theory but similar to the SM Higgs boson would be accessible, even if the cross section times branching ratio is lower than that of the SM Higgs boson.

We arrive at a the cross-section limit by repeating the above-mentioned hypothesis-testing procedure with an increasing multiplication factor of the signal, until a $95 \% \mathrm{CL}_{\mathrm{S}}$ exclusion is achieved.

The limit on the cross section is of course calculated for the observed data, but it is also determined for the median of the background-only model. This can be interpreted as the expected limit if the data follows the background-only model perfectly. 


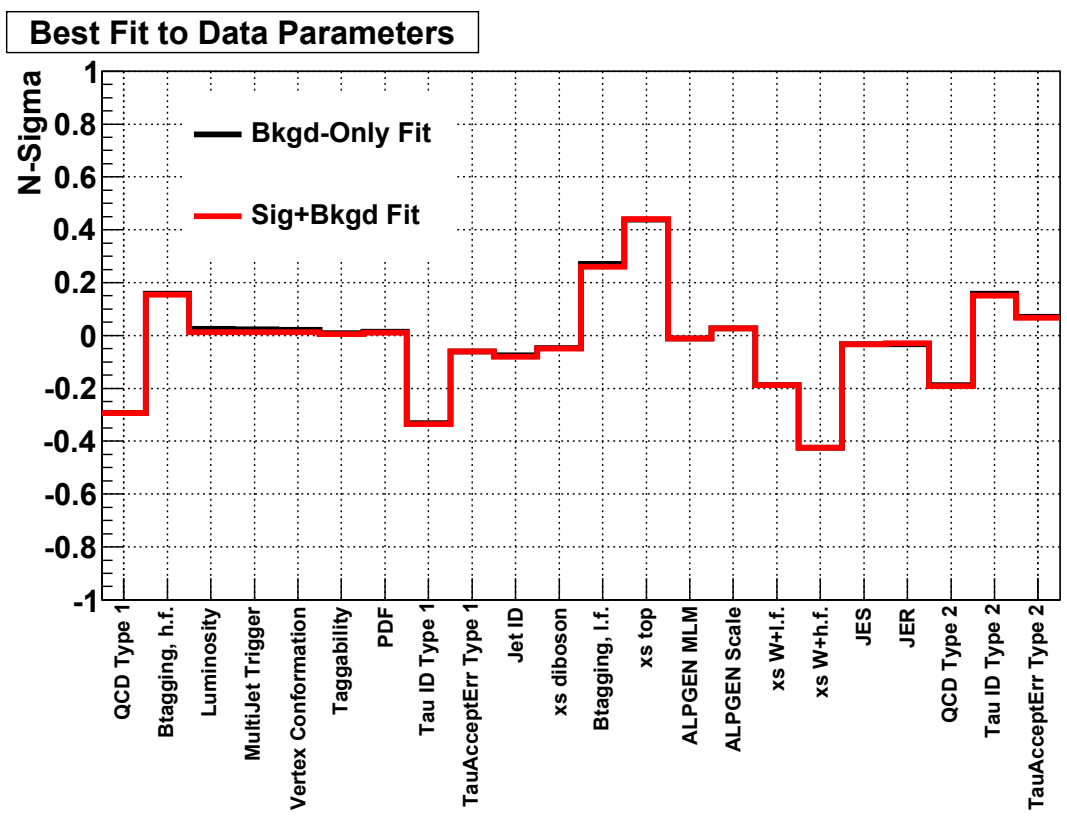

Figure 5.6: variation (measured in standard deviations) of each uncertainty after fitting in the background only (black) and signal + background (red) hypotheses. The used Higgs mass is $115 \mathrm{GeV}$.

\subsection{Results}

\subsubsection{Nuisance Parameter Fit}

It is important to check that the nuisance-parameter fit of Equation (5.21) makes sense before presenting the limit calculation. Therefore, a number of tests are performed to look at the effect of each systematic uncertainty. The pull of each systematic is looked at individually and the amount of standard deviations $\sigma$ that each systematic uncertainty changes under fitting for the signal and background is considered. The systematics that are shown to fluctuate the most under fitting are the tau identification uncertainty and the cross sections for the important processes left after $b$-tagging: $W+$ heavy flavor and $t \bar{t}$. These uncertainties are large in magnitude and affect the integrated number of background events as the fit tries to equalize the data and background model as much as possible.

The fitted nuisance parameters from the observed data fit compared to the nominal non-fitted distributions are shown in Figure 5.6 for both hypotheses. The 
two hypotheses are very comparable, so the difference between their fits to data is very small. No fitted systematic uncertainty shows more than a $0.5 \sigma$ deviation. This is highly unlikely for 22 parameters, so it is an indication that the originally estimated uncertainties were probably too large.

Another reason why the distribution of fitted nuisance parameters is so narrow is that most systematics have a small impact on the total uncertainty and are often correlated with uncertainties that have a greater impact. For example the uncertainty on the diboson cross section is negligible compared to the one on the Heavy Flavor Ratio. Therefore, the impact of a diboson-cross-section deviation on the fit is very small and Equation (5.21) is minimized mainly by reducing $S^{2}$ to a minimum.

The $t \bar{t}$ cross section is fitted to be higher than nominal, while the cross section of $W+$ heavy flavor appears to be lower than expected. This is probably the result of a slight overall data deficit in 2-jet channels and data overshoot in 3 -jet channels. Because the $t \bar{t}$ events are a more prominent background in the 3 -jet channels, the observed fit is expected qualitatively.

In Figure 5.7, the fit-to-measured-data results for the background-only hypothesis are incorporated in the BDT-output background estimates of the different channels. They are plotted along with the nominal background estimates and the measured data points.

\subsubsection{Confidence Level Limits}

For $m_{H}=115 \mathrm{GeV}$, the LLR distributions for both hypotheses can be seen in Figure 5.8 The measured data value is also shown. The LLR distributions for all Higgs masses with fitted nuisance parameters are pictured in Figure 5.9. In this figure, for each $m_{H}$ the data LLR is plotted, together with the LLRs of the background and signal expectations. The $\pm 1 \sigma$ and $\pm 2 \sigma$ bands contain $68 \%$ and $95 \%$ of the pseudo-experiment outcomes, respectively.

As neither the signal plus background nor the background-only hypothesis are rejected by the the data, limits for the cross section times branching ratio of the Higgs signal are set. Initially, limits are set with no systematic uncertainties. For the final result, the nuisance-parameter fitting method is used.

The $\left(1-\mathrm{CL}_{\mathrm{S}}\right)=95 \%$ limits obtained for the $W H / Z H$ cross section relative to their Standard-Model prediction are shown in Table 5.4 and Figure 5.10 as function of the Higgs mass. The observed limits are lower than the expected limits. This is consistent with the fact that a slight data deficit is observed compared to the predicted background in the high BDT output region for the most sensitive channel (two $b$-tagged, type 2 tau with 2 associated jets). 


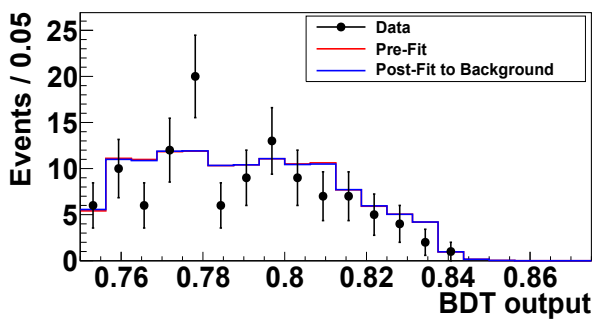

(a) 2 b-tag, tau type 2, 2 jets

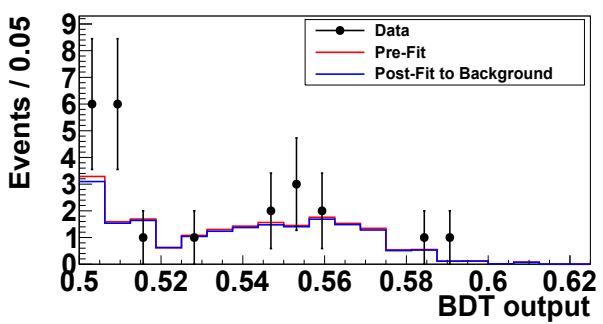

(c) 2 b-tag, tau type 1, 2 jets

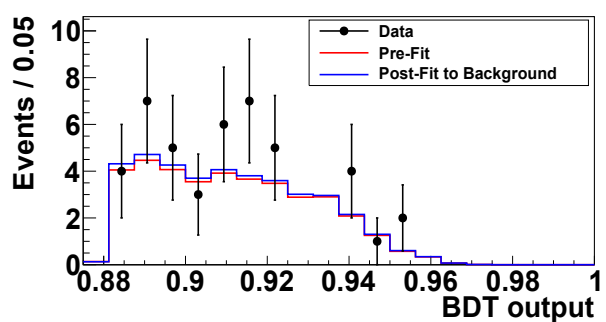

(e) 2 b-tag, tau type 2,3 jets

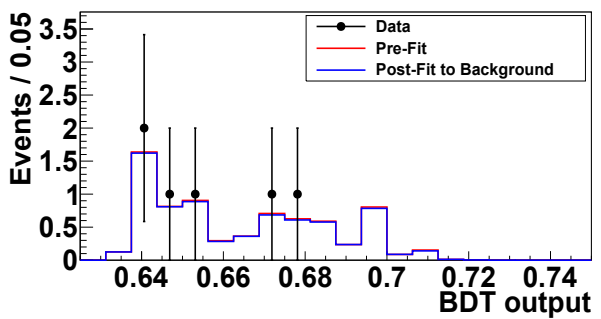

(g) 2 b-tag, tau type 1,3 jets

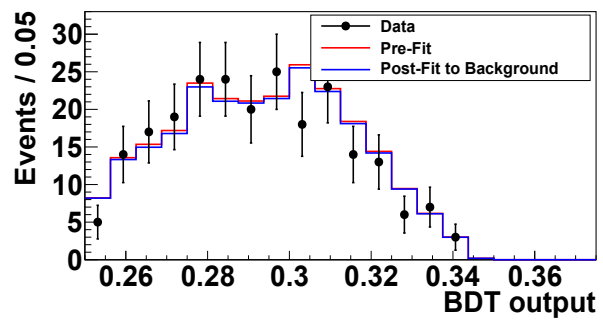

(b) $1 b$-tag, tau type 2,2 jets

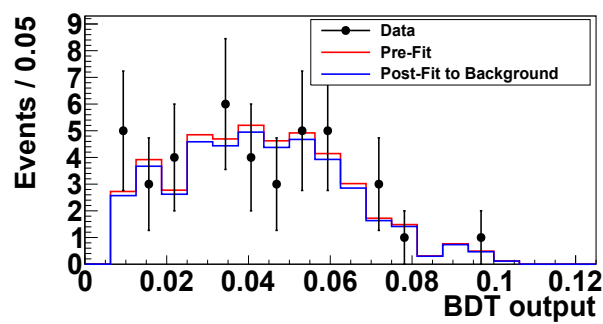

(d) 1 b-tag, tau type 1,2 jets

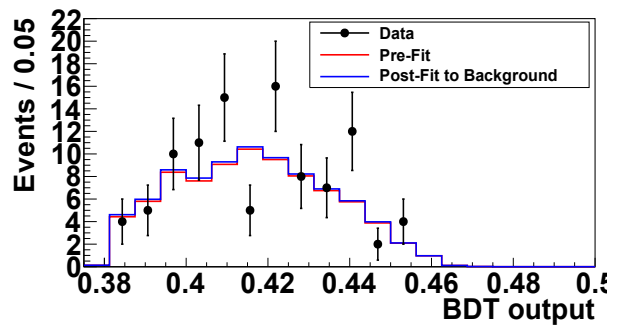

(f) $1 b$-tag, tau type 2,3 jets

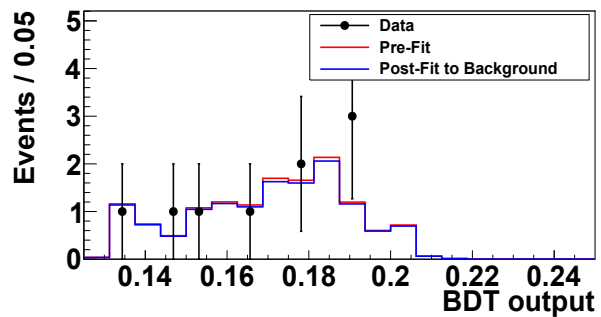

(h) 1 b-tag, tau type 1,3 jets

Figure 5.7: the BDT output estimates before (red) and after the fit to the measured data (blue) in the 8 channels for the background hypothesis and $m_{H}=115 \mathrm{GeV}$. 


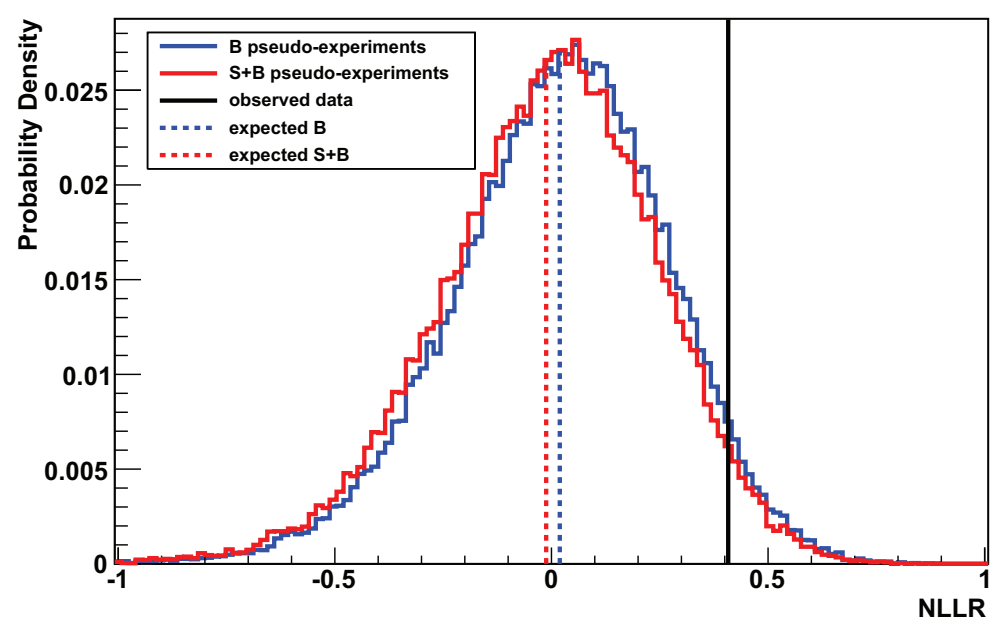

Figure 5.8: LLR distribution for $m_{H}=115 \mathrm{GeV}$.

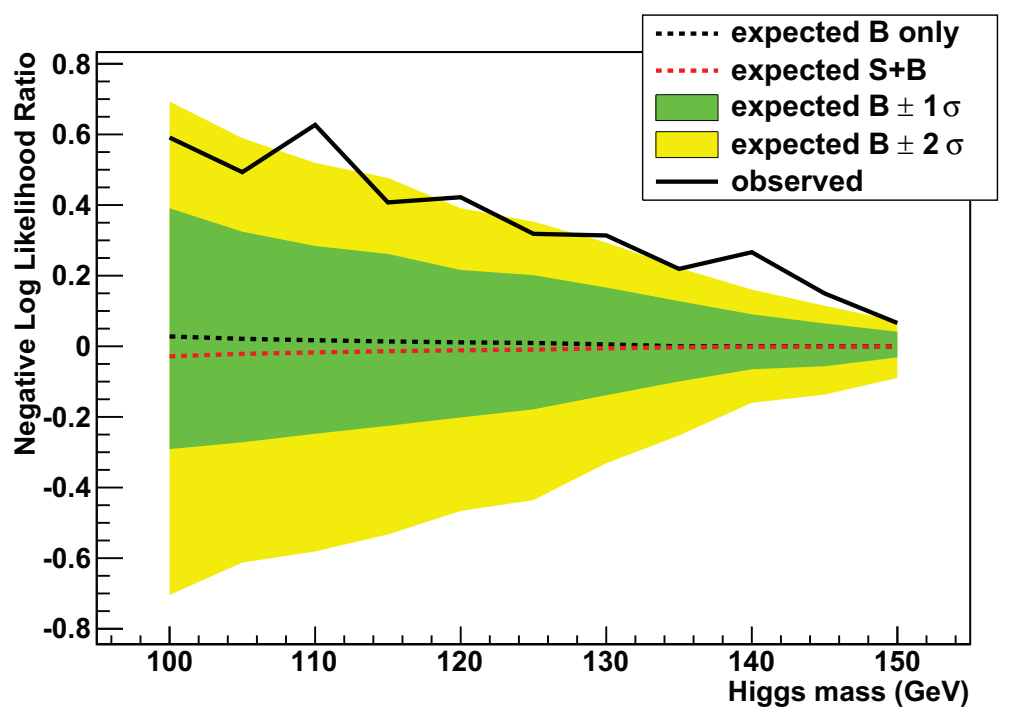

Figure 5.9: LLR distribution for the final result using fitted systematic uncertainties. 


\begin{tabular}{crrrr}
\hline & \multicolumn{2}{c}{ no uncertainties } & \multicolumn{2}{c}{ fitted systematics } \\
$m_{H}(\mathrm{GeV})$ & expected & observed & expected & observed \\
\hline 100 & 10.0 & 6.2 & 16.1 & 10.9 \\
105 & 12.0 & 8.1 & 17.0 & 12.3 \\
110 & 12.9 & 8.6 & 19.7 & 8.7 \\
115 & 16.2 & 10.0 & 21.7 & 11.7 \\
120 & 17.1 & 11.2 & 22.5 & 11.1 \\
125 & 19.6 & 12.9 & 25.6 & 14.7 \\
130 & 20.6 & 15.6 & 37.3 & 30.1 \\
135 & 31.6 & 21.3 & 50.3 & 46.9 \\
140 & 40.6 & 30.7 & 64.2 & 48.8 \\
145 & 58.3 & 45.6 & 94.6 & 69.3 \\
150 & 101.3 & 74.9 & 159.4 & 92.1 \\
\hline
\end{tabular}

Table 5.4: expected and observed 95\% CL limits in terms of the SM cross section for the specified Higgs-boson mass hypotheses, using methods without uncertainties and with fitted systematics.

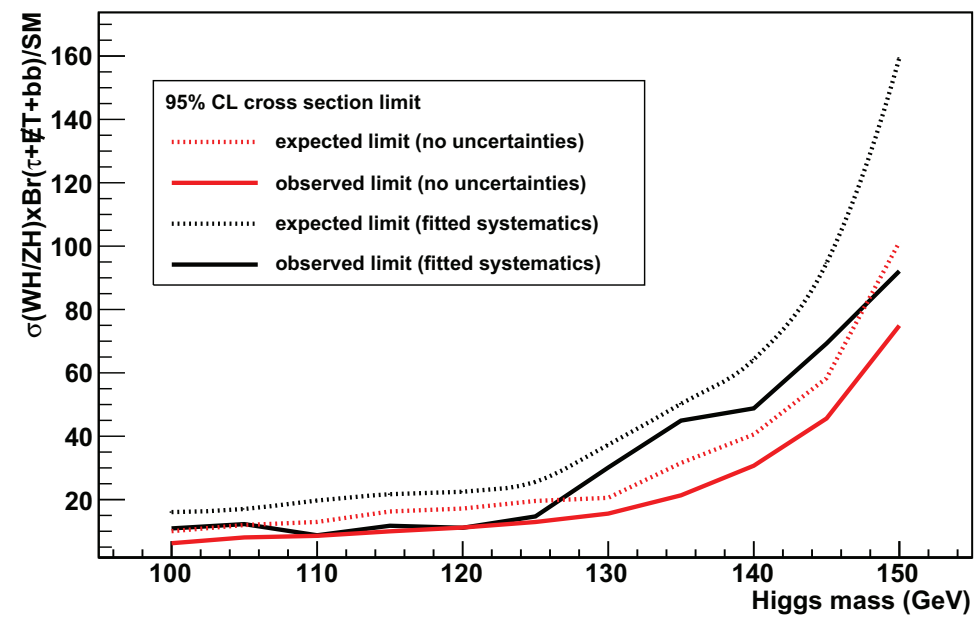

Figure 5.10: limits obtained for the $W H / Z H$ processes relative to the Standard Model cross section at the $95 \%$ confidence level for each of the limit setting methods. 


\section{Chapter 6}

\section{Conclusion}

In this chapter, the analysis of Chapters 4 and 5 is placed in a wider context. First, we will take a critical look at the $W H \rightarrow \tau \nu b \bar{b}$ analysis itself. Then, the combination of several channels into a single $\mathrm{D} \emptyset$ or Tevatron result is discussed. Finally, we will touch upon recent results of the SM Higgs searches at the Large Hadron Collider (LHC) experiments.

\subsection{Discussion on $W H \rightarrow \tau \nu b \bar{b}$}

The analysis presented in this thesis builds on the first search for the SM Higgs boson using hadronic tau decays at a hadron collider [106], which was performed on $1.0 \mathrm{fb}^{-1}$ of recorded data. The updated $W H \rightarrow \tau \nu b \vec{b}$ analysis uses an integrated luminosity of $4.0 \mathrm{fb}^{-1}$, a much larger dataset.

Next to the increase in luminosity, the new analysis also has more data due to a looser $\mathbb{E}_{\mathrm{T}}$ cut: it was lowered from 30 to $15 \mathrm{GeV}$. On the other hand, the higher instantaneous luminosities have reduced the tau-identification efficiencies for Run IIb data.

Also the $b$-tagging is looser in the new analysis; the $1.0 \mathrm{fb}^{-1}$ result used one asymmetric $b$-tag sample [90, with events where one jet has a tight $b$-tag, and another jet has a loose tag. The updated analysis uses two samples: one containing events with two loosely $b$-tagged jets, and another with events where one jet was tightly $b$-tagged and the other explicitly failed the loose criterion.

Compared to the first result, the multijet background estimation was improved, by requiring that the multijet contribution to the background is larger than or equal to zero. This is done both in large regions of the examined variables as well as bin-by-bin.

Another improvement comes from using boosted decision trees to get the final 


\begin{tabular}{lcccrrr}
\hline version & $L\left(\mathrm{fb}^{-1}\right)$ & background & signal & data & exp. limit & obs. limit \\
\hline Run IIa & 1.0 & $22.8 \pm 0.9$ & 0.15 & 13 & 42 & 35 \\
Run IIa \& b & 4.0 & $578 \pm 2.3$ & 1.71 & 570 & 22 & 12 \\
\hline
\end{tabular}

Table 6.1: comparison with the previous version of the $W H \rightarrow \tau \nu b \bar{b}$ analysis. The quoted number of background, signal and recorded events are summed over all sub-channels of the analysis. The observed and expected limits as well as the signal contribution are listed for $m_{H}=115 \mathrm{GeV}$.

variable on which the hypothesis is tested; the $1.0 \mathrm{fb}^{-1}$ result was based only on $m_{\mathrm{jj}}$. The dijet invariant mass is still the most important input to the decision trees, but other variables have a non-negligible impact.

The analysis in this thesis also includes a more complete treatment of the shape systematics. In the version of [77, the shape difference a systematical error introduces in the BDT output distribution was considered to be dominantly coming from the altered dijet invariant mass. In the current analysis, also the shape difference in the other, lower-impact variables are taken into account when determining the BDT-output fluctuations.

Furthermore, the overshoot of the MC estimation in the sideband region for both the MC control sample and the signal sample is addressed in the new version, along with the treatment of negative bins coming from statistical fluctuations.

A brief efficiency and limit comparison between the two analysis versions can be seen in Table 6.1 .

\subsubsection{Possible Further Improvements}

One improvement is updating the data-set to the full $10.7 \mathrm{fb}^{-1}$ that was recored by D $\varnothing$ during Run II. Naively, this would give an improvement for the $s / \sqrt{b}$ of a factor $\sqrt{10.7 / 4.0} \approx 1.6$. An improvement of the same factor could be expected for the cross-section limits of Table 5.4 and Figure 5.10 .

Also a reduction in the systematic uncertainties can enhance the sensitivity greatly. Combined, the systematics degrade the cross section limit from 16.2 to 21.7 times the SM cross section for a Higgs mass of $115 \mathrm{GeV}$.

The largest systematic is the $20 \%$ uncertainty on the ratio between $W / Z+$ h.f. and $W / Z+$ l.f., so between a vector boson with heavy partons $(c$ or $b$ ) and one with light partons $(g, u, d, s)$. This systematic is limited by the lack of theoretical understanding of the $W / Z+$ h.f. cross-section. Therefore, only theoretical improvements of the $W / Z+$ h.f. predictions would directly enhance the sensitivity of the $W H \rightarrow \tau \nu b \bar{b}$ analysis.

The estimation of the multijet background remains a challenge. A smaller 


\begin{tabular}{llllll}
\hline channel & $L$ & channel & $L$ & channel & $L$ \\
\hline$H \rightarrow W^{+} W^{-} \rightarrow \ell^{+} \nu \ell^{-} \bar{\nu}$ & 8.1 & $V H \rightarrow \ell^{+} \ell^{-}+X$ & 5.3 & $Z H \rightarrow \nu \nu b \bar{b}$ & 8.4 \\
$H \rightarrow W^{+} W^{-} \rightarrow \ell \nu q \bar{q}$ & 5.4 & $H+X \rightarrow \ell^{ \pm} \tau^{\mp} j j$ & 4.3 & $Z H \rightarrow l \bar{l} b \bar{b}$ & 8.6 \\
$H+X \rightarrow \mu^{ \pm} \tau^{\mp}+\leq 1$ jet & 7.3 & $W H \rightarrow \ell \nu b \bar{b}$ & 8.5 & $H \rightarrow \gamma \gamma$ & 8.2 \\
\hline
\end{tabular}

Table 6.2: channels of the DØ combination of July 2011 with their integrated luminosities $L$ in $\mathrm{fb}^{-1}$ [107.

uncertainty on this background should be attainable; one could for example utilize other sideband regions, such as the regions $3.5<\mathbb{E}_{\mathrm{T}}^{\mathrm{SIG}}<4.5$ and $\Delta \phi\left(\not p_{\mathrm{T}}^{\mathrm{trk}}, \mathbb{E}_{\mathrm{T}}\right)>$ 2. It might also be useful to see what the relation is between MC-generated QCD in the $0.3<\mathrm{NN}_{\tau}<0.7$ and the $\mathrm{NN}_{\tau}>0.9$ region.

Ensuring orthogonality with the $Z H \rightarrow \nu \nu b \bar{b}$ channel would also be a welcome modification, in order for the $W H \rightarrow \tau \nu b \bar{b}$ channel to be included a D $\varnothing$ combination. This is discussed in more detail in Section 6.2.1.

\subsection{The DØ Combination}

The DØ experiment combines several SM Higgs-boson analyses to arrive at a single DØ measurement of the LLR together with limits on SM-Higgs-boson production. The latest combination [107] includes the channels listed in Table 6.2.

This combined measurement uses mass points from 100 to $200 \mathrm{GeV}$, with $5 \mathrm{GeV}$ intervals. For each Higgs mass, the nuisance-parameter fit described in Section 5.3 .2 is performed on the different channels simultaneously. This is done in the same way as the combination of the eight subchannels of the $W H \rightarrow \tau \nu b \bar{b}$ analysis: global systematic uncertainties, such as the luminosity or the $W+$ jets cross section, are assumed fully correlated across the different channels, while other systematics are channel specific and are taken to be fully independent.

The log likelihood ratio of the July 2011 D $\varnothing$ combination can be seen in Figure 6.1(a) The corresponding 95\%-CL limits are shown in Figure6.1(b). In the latter figure it can be seen that a $161 \mathrm{GeV}<m_{H}<170 \mathrm{GeV}$ SM Higgs boson is excluded by the $\mathrm{D} \varnothing$ experiment with a $95 \% \mathrm{CL}$.

\subsubsection{Including the $W H \rightarrow \tau \nu b \bar{b}$ Channel}

In order to include the $W H \rightarrow \tau \nu b \bar{b}$ analysis, it should be independent from the other analyses in the $\mathrm{D} \varnothing$ combination.

In Section 4.2.3 there is a description of how orthogonality with the $W H \rightarrow$ $\ell \nu b \bar{b}$ and $H+X \rightarrow \ell^{ \pm} \tau^{\mp} j j$ analyses is ensured. Unfortunately, orthogonality with 
the $Z H \rightarrow \nu \nu b \bar{b}$ analysis cannot easily be achieved; all the physics requirements of the $\nu \nu b \bar{b}$ final state, missing transverse energy and $2 b$-jets, are also present in $\tau \nu b \bar{b}$. The latter has a hadronic tau in addition, but the $Z H \rightarrow \nu \nu b \bar{b}$ analysis does not veto on this hadronic tau.

A study has been performed to check the overlap between the $\tau \nu b \bar{b}$ and the $\nu \nu b \bar{b}$ channels. This is done by ascertaining which measured data events of the $\tau \nu b \bar{b}$ analysis were also used for $\nu \nu b \bar{b}$. Removing these events is not enough to ensure orthogonality; the overlap in MC generated events also needs to be removed. However, the coincidences between the two channels in data do provide an estimate of the amount of the overall overlap.

In total, 132 out of $570 \tau \nu b \bar{b}$ measured data events were also used in the $Z H \rightarrow \nu \nu b \bar{b}$ analysis, which is $23 \pm 2 \%$. This ratio is similar for all subchannels, as is listed in Table 6.3

The overlapping events have been plotted on the BDT output of the two most important $\tau \nu b \bar{b}$ subchannels in Figure 6.2. The overlapping events do not appear to be more signal or background-like than the rest of the data.

\subsection{Tevatron Combination}

Similarly to the $\mathrm{D} \varnothing$ combination, the CDF channels are combined with the $\mathrm{D} \varnothing$ ones 93. In this combination, orthogonality between channels of different detectors is automatically achieved, because the datasets are statistically independent. The correlations between the systematic uncertainties do have to be taken into account: shared uncertainties such as the $W+$ jets cross section are fully correlated,
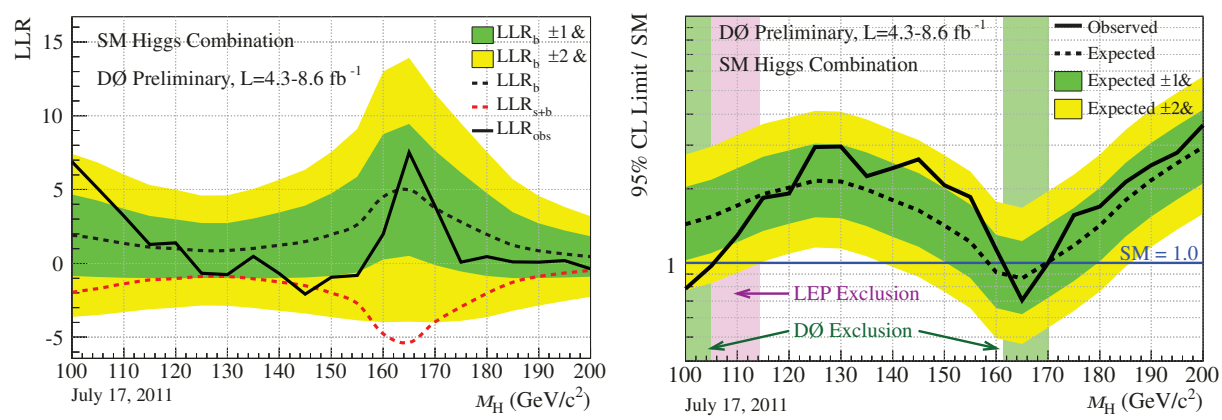

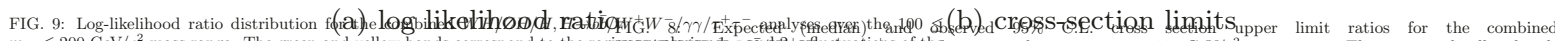

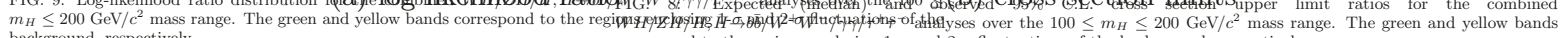

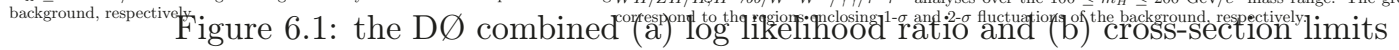
at the $95 \%$ confidence level [107. Next to the observed limit, the median expected limit with error band is also shown. 


\begin{tabular}{lrrl}
\hline$\tau \nu b \bar{b}$ subchannel & $\tau \nu b \bar{b}$ events & overlap events & ratio (\%) \\
\hline one $b$-tag, tau type 2, 2 jets & 232 & 46 & $20 \pm 3$ \\
one $b$-tag, tau type 2, 3 jets & 99 & 27 & $28 \pm 6$ \\
one $b$-tag, tau type 1, 2 jets & 40 & 11 & $30 \pm 10$ \\
one $b$-tag, tau type 1, 3 jets & 9 & 2 & $33 \pm 24$ \\
two b-tag, tau type 2, 2 jets & 117 & 27 & $24 \pm 5$ \\
two b-tag, tau type 2, 3 jets & 44 & 12 & $30 \pm 9$ \\
two b-tag, tau type 1, 2 jets & 23 & 7 & $35 \pm 15$ \\
two b-tag, tau type 1, 3 jets & 6 & 0 & $17 \pm 20$ \\
all subchannels combined & 570 & 132 & $23 \pm 2$ \\
\hline
\end{tabular}

Table 6.3: the amount of data events present in both the $W H \rightarrow \tau \nu b \bar{b}$ and $Z H \rightarrow$ $\nu \nu b \bar{b}$ analyses for the different $W H \rightarrow \tau \nu b \bar{b}$ subchannels.

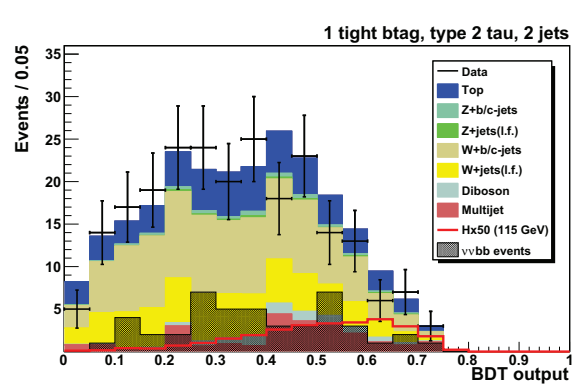

(a) BDT output 1 b-tag, tau type 2, 2 jets

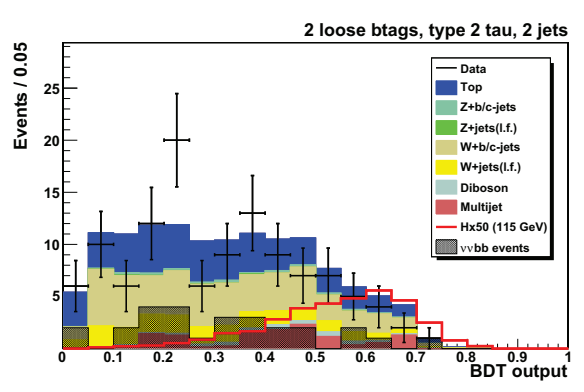

(b) BDT output 2 b-tag, tau type 2,2 jets

Figure 6.2: the overlap of $\nu \nu b \bar{b}$ events in the leading two $\tau \nu b \bar{b}$ subchannels.

while others are channel or detector specific.

The LLR results of the Tevatron combination are shown in Figure6.3. It is clear that at Higgs masses around $165 \mathrm{GeV}$, the observed LLR follows the expectation of the background hypothesis. At $m_{H} \approx 130 \mathrm{GeV}$, a $1.4 \sigma$ signallike fluctuation is observed. The probability for this to occur if the background hypothesis is true, or $1-\mathrm{CL}_{\mathrm{B}}$, is about $8 \%$. Although in the future this might prove to be a hint of a Higgs boson at that mass, $8 \%$ is too high to conclude that the Higgs boson exists; the 'industry standards' for claiming evidence and discovery are $1-\mathrm{CL}_{\mathrm{B}}<1.3 \cdot 10^{-3}$ and $1-\mathrm{CL}_{\mathrm{B}}<2.9 \cdot 10^{-7}$, respectively, corresponding to $3 \sigma$ and $5 \sigma$ deviations from the background-only hypothesis.

In Figure 6.4 the cross-section limits are shown for the Tevatron combination. 
This measurement excludes at $95 \%$ confidence level a SM Higgs boson with a mass in the region $156 \mathrm{GeV}<m_{H}<177 \mathrm{GeV}$. The region around $m_{H} \approx 130 \mathrm{GeV}$ has a higher than expected cross-section limit, which corresponds to the lower LLR value discussed before.

The Standard-Model fit of Figure 1.6 is also calculated with the Tevatron results included, as seen in Figure 6.5. It shows the $\chi^{2}$ that a certain fixed Higgs mass would add to the fit, compared to the best fitting Higgs mass. The fit also includes direct searches at LEP, which are discussed on page 14

\subsubsection{Tevatron Prospects}

The Tevatron combination of Figure 6.4 used datasets with up to $8.6 \mathrm{fb}^{-1}$ integrated luminosity. It is expected that in the summer of 2012 a Tevatron combination using the full $10.7 \mathrm{fb}^{-1}$ dataset will be published. The projection for the Tevatron limits is shown in Figure 6.6 [108]. Also possible analysis improvements, like advancements in object reconstruction, are taken into account. It can be seen that at around $10 \mathrm{fb}^{-1}$ the expected limit of the Tevatron drops below the SM cross section for $m_{H}=115 \mathrm{GeV}$, even without substantial analysis improvements.

\subsection{Large Hadron Collider Results}

In 2011, the Large Hadron Collider (LHC) has started to become a serious competitor to the Tevatron. There are two experiments at the LHC that are important to a direct search for a SM Higgs boson: the Atlas (A Toroidal LHC ApparatuS) and CMS (Compact Muon Solenoid) experiments. These record proton-proton collisions, which have a current center-of-mass energy of $7 \mathrm{TeV}$.

The LHC has a much higher probability than the Tevatron to produce a Higgs boson in a collision: for associated vector boson production $(q \bar{q} \rightarrow V H)$ the cross section is about 5 times that of the Tevatron, for gluon fusion $(g g \rightarrow H)$ and vector boson fusion $(q q \rightarrow V V q q \rightarrow H q q)$ it is roughly a factor of 20 higher. This is mainly caused by the larger number of proton constituents that carry enough energy to produce heavy particles. However, the background cross section also increases greatly. The result is that the associated vector boson production, which is dominant for low $m_{H}$, has a similar $s / \sqrt{b}$ as the Tevatron. For gluon fusion and vector boson fusion, which are important at $m_{H}>140 \mathrm{GeV}$, the $s / \sqrt{b}$ is much higher for the LHC.

Like the Tevatron combination, an LHC combined SM Higgs boson search has been performed on up to $2.3 \mathrm{fb}^{-1}$ of data [109. The resulting cross section limits can be seen in Figure 6.7. Most of the region allowed by the SM fit of Figure 1.6 is excluded at $95 \%$ confidence level. If the SM Higgs boson exists, it has a mass between 114.4 and $141 \mathrm{GeV}$. 


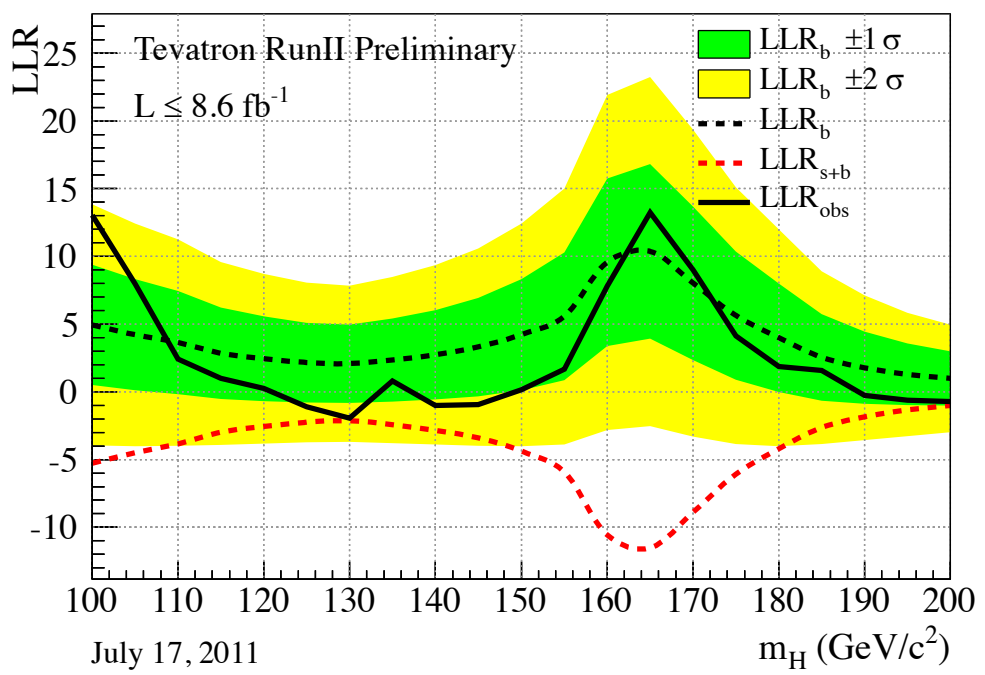

Figure 6.3: the LLR for the Tevatron channels combined, as a function of the hypothesized Higgs mass $m_{H}$ [93].

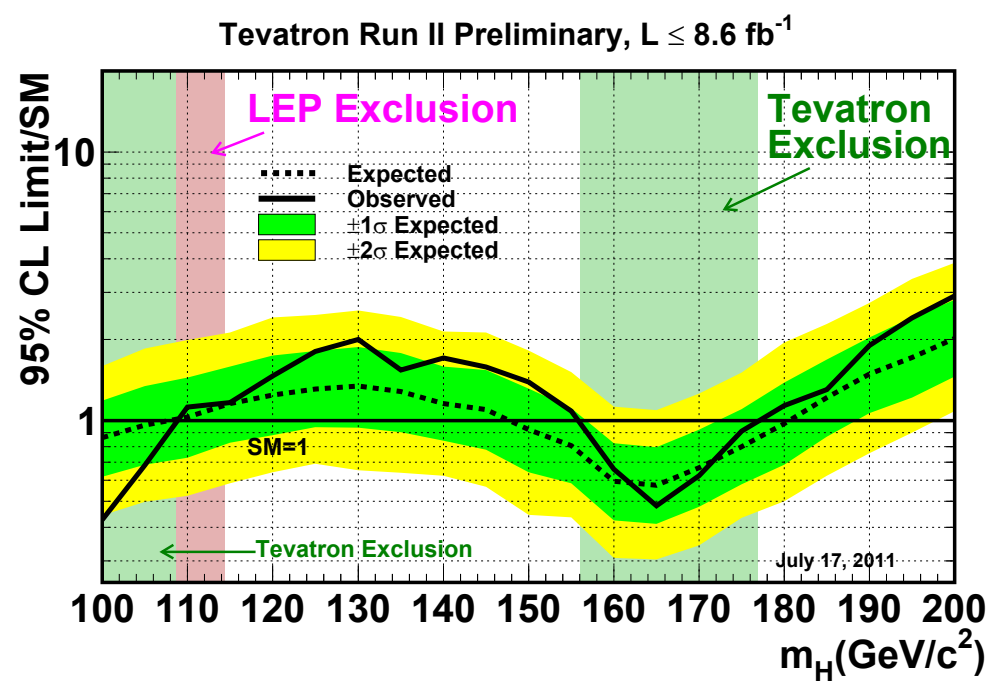

Figure 6.4: cross-section limits obtained for the combined Tevatron channels, relative to the Standard Model cross section at 95\% confidence level 93 . Next to the observed limit, the expected limit with error band is also shown. 


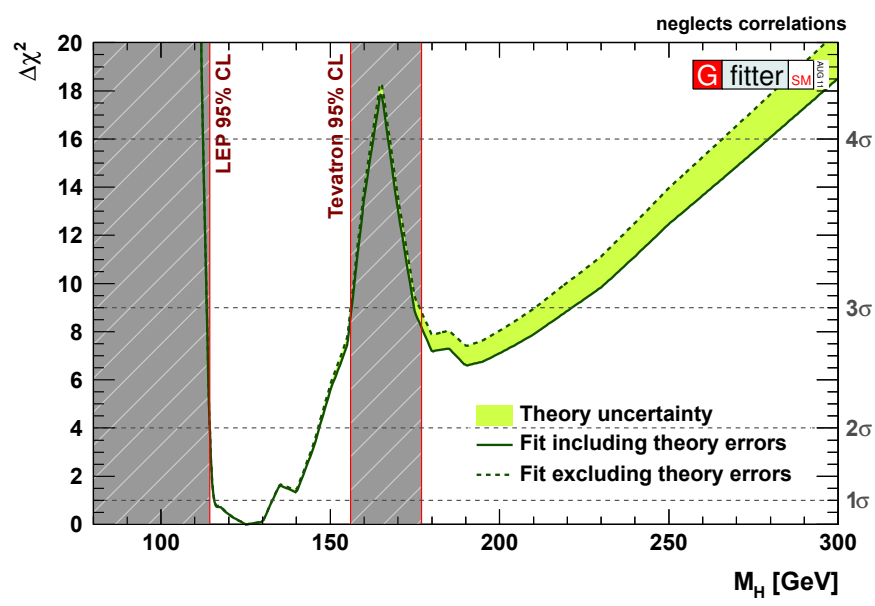

Figure 6.5: the difference in $\chi^{2}$ from the SM fit, including LEP and TeVatron search results, as a function of a fixed Higgs mass in the fit [18. The shaded regions represent the regions excluded by LEP and Tevatron experiments.

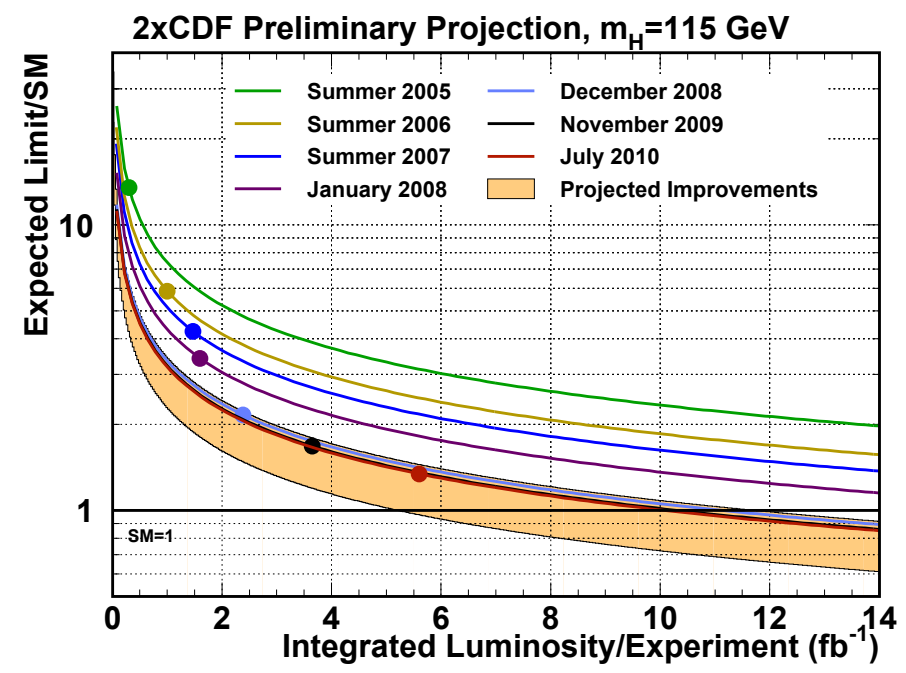

Figure 6.6: the estimated cross section limits for the Tevatron combination for $m_{H}=115 \mathrm{GeV}[108$. This projection by the CDF collaboration is done by taking 2 times the CDF result as model for the Tevatron combination. The colored lines are $1 / \sqrt{L}$ curves with $L$ the integrated luminosity. 


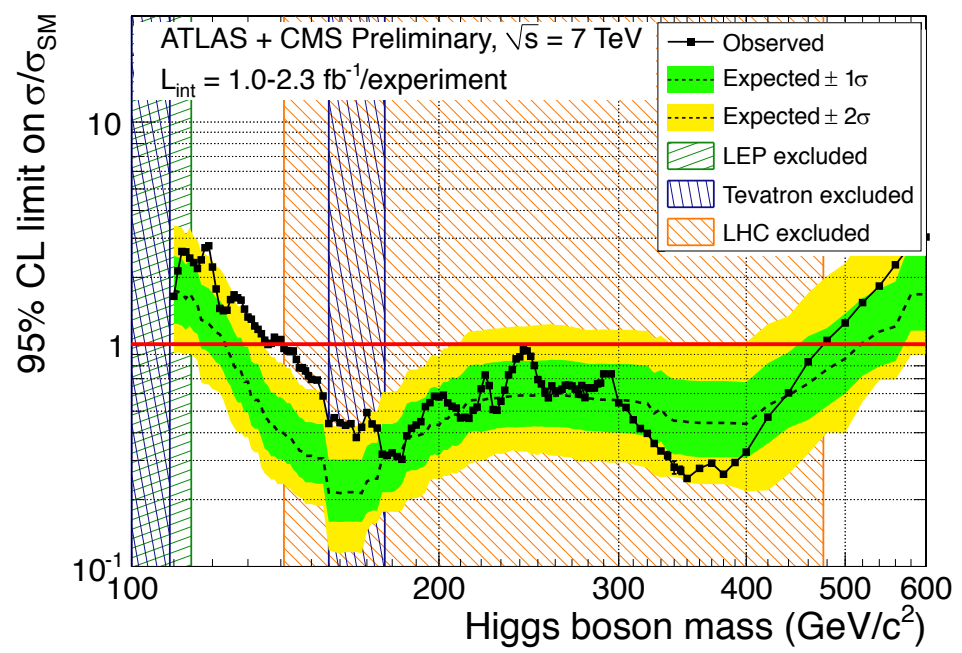

Figure 6.7: cross section limits obtained for the $L \leq 2.3 \mathrm{fb}^{-1}$ LHC dataset 109].

Atlas and CMS have each recorded about $5.2 \mathrm{fb}^{-1}$ of data in 2011 and for the next year, a total dataset of $15 \mathrm{fb}^{-1}$ per experiment is foreseen. This means that there is a good prospect to find the SM Higgs boson, or evidence for it, in 2012.

Even if the Standard Model Higgs boson is conclusively excluded at the LHC, supersymmetric versions can still exist [110]. Furthermore, some processes' cross sections need to deviate from the SM (without Higgs boson) predictions because of unitarity. An example is the cross section of $W_{L}^{+} W_{L}^{-} \rightarrow Z_{L} Z_{L}$ in Figure 1.3

In any case — whether the Higgs boson is found or not — we live in interesting times... 


\section{Bibliography}

[1] S. Glashow, Partial-symmetries of weak interactions,

Nucl. Phys. 22 p. 579-588 (1961) — Cited on pages 3 and 7

[2] A. Salam and J. Ward,

Phys. Lett. 13 p. 168 (1964) - Cited on pages 3 and 7.

[3] S. Weinberg, A Model of Leptons,

Phys. Rev. Lett. 19 p. 1264-1266 (1967) - Cited on pages 3 and 7.

[4] G. 't Hooft and M. Veltman, Regularization and renormalization of gauge fields,

Nucl. Phys. B44 p. 189-213 (1972) - Cited on page 3.

[5] M. Maltoni et al., Status of global fits to neutrino oscillations,

New J. Phys. 6 p. 122 (2004) - Cited on page 4.

[6] K. Nakamura et al. (Particle Data Group),

J. Phys. G37 p. 075021 (2010) - Cited on pages 5 , 9 and 65.

[7] R. Feynman, Space-Time Approach to Quantum Electrodynamics,

Phys. Rev. 76 p. 769-789 (1949) — Cited on page 5.

[8] F. Englert and R. Brout, Broken symmetry and the mass of gauge vector mesons,

Phys. Rev. Lett. 13 p. 321-323 (1964) — Cited on page 7.

[9] P. W. Higgs, Broken symmetries, massless particles and gauge fields,

Phys. Lett. 12 p. 132-133 (1964) — Cited on page 7.

[10] P. W. Higgs, Broken symmetries and the masses of gauge bosons,

Phys. Rev. Lett. 13 p. 508-509 (1964) — Cited on page 7.

[11] G. Guralnik, C. Hagen and T. Kibble, Global conservation laws and massless particles,

Phys. Rev. Lett. 13 p. 585-587 (1964) — Cited on page 7 
[12] P. W. Higgs, Spontaneous symmetry breakdown without massless bosons, Phys. Rev. 145 p. 1156-1163 (1966) — Cited on page 7.

[13] T. Kibble, Symmetry breaking in non-Abelian gauge theories, Phys. Rev. 155 p. 1554-1561 (1967) — Cited on page 7.

[14] R. Kleiss and G. van den Oord, CAMORRA: $A++$ library for recursive computation of particle scattering amplitudes,

Comp. Phys. Comm. 182 p. 435-447 (2010)

- Cited on pages 11 and 12 .

[15] J. M. Cornwall et al., Derivation of gauge invariance from high-energy unitarity bounds on the $S$ matrix,

Phys. Rev. D10 p. 1145-1167 (1974) — Cited on page 11

[16] C. Kolda and H. Murayama, The Higgs mass and new physics scales in the minimal standard model,

JHEP 07 p. 035 (2000) - Cited on page 12

[17] J. Ellis et al., The Probable Fate of the Standard Model,

Phys. Lett. B679 p. 369-375 (2009) — Cited on pages 13 and 14

[18] M. Baak et al. (The Gfitter Group), Updated Status of the Global Electroweak Fit and Constraints on New Physics,

Eur. Phys. J. C60 p. 543 (2009) - Cited on pages 13, 15 and 138.

[19] LEP Working Group for Higgs Boson Searches, Search for the Standard Model Higgs Boson at LEP,

Phys. Lett. B565 p. 61-75 (2003) - Cited on page 14

[20] V. Ravindran, J. Smith and W. van Neerven, NNLO corrections to the total cross section for Higgs boson production in hadron-hadron collisions,

Nucl. Phys. B665 p. 325-366 (2003) - Cited on page 16.

[21] A. Djouadi, J. Kalinowski and M. Spira, HDECAY: a Program for Higgs Boson Decays in the Standard Model and its Supersymmetric Extension,

Comp. Phys. Comm. 108 p. 56-74 (1998) — Cited on page 16.

[22] The DØ Collaboration, Search for High Mass Top Quark Production in $p \bar{p}$ Collisions at $\sqrt{s}=1.8 \mathrm{TeV}$,

Phys. Rev. Lett. 74 p. 2632-2637 (1995) - Cited on page 19

[23] Tevatron Operations, Rookie Books (January 6, 2010),

http://www-bdnew.fnal.gov/operations/rookie_books/rbooks.html

- Cited on page 20 . 
[24] The D $\varnothing$ Collaboration, The upgraded DØ detector,

Nucl. Instr. Meth. A565 p. 463-537 (2006) - Cited on page 23.

[25] The DØ Collaboration, The DØ Silicon Microstrip Tracker,

Nucl. Instr. Meth. A634 p. 8-46 (2011) - Cited on page 26

[26] R. Angstadt et al., The Layer 0 Inner Silicon Detector of the DØ Experiment,

Nucl. Instr. Meth. A622 p. 298 (2010) - Cited on page 27.

[27] M. Adams et al., Design Report of the Central Preshower Detector for the DØ Upgrade,

DØ note 3014 (1996) — Cited on page 30.

[28] A. Gordeev et al., Technical Design Report of the Forward Preshower Detector for the DØ Upgrade,

DØ note 3445 (1998) — Cited on page 30.

[29] T. Sjöstrand et al., PYTHIA 6.2, Physics and Manual, [hep-ph/0108264v1] (2001) — Cited on page 40.

[30] M. Mangano et al., ALPGEN, a generator for hard multiparton processes in hadronic collisions,

JHEP 307 p. 001 (2003) — Cited on page 40.

[31] J. Campbell and K. Ellis, MCFM - Monte Carlo for FeMtobarn processes, http://mcfm.fnal.gov/ - Cited on page 40.

[32] A. Pukhov et al., CompHEP - a package for evaluation of Feynman diagrams and integration over multi-particle phase space, [hep-ph/9908288v2] (2000) — Cited on page 40.

[33] Y. Fisyak and J. Womersley, Dogstar DØ GEANT Simulation of the Total Apparatus Response,

DØ note 3191 (1997),

http://www-d0.fnal.gov/d0dist/dist/releases/development/ d0gstar/docs/html/d0gstar.html - Cited on page 40.

[34] CERN Application Software Group, GEANT, wwwasdoc.web.cern.ch/wwwasdoc/pdfdir/geant.pdf

- Cited on page 40 .

[35] Q. Li and S. Protopopescu, DoSim, WwW-d0.fnal.gov/computing/MonteCarlo/simulation/d0sim.html

- Cited on page 40 . 
[36] D. O'Neil and A. Bellavance, Trigsim Documentation, http://www-d0.fnal.gov/computing/trigsim/general/docs.html - Cited on page 40 .

[37] H. Greenlee, The DØ Kalman Track Fit, DØ note 4303 (2004) — Cited on page 43.

[38] G. Hesketh, Central Track Extrapolation Through the DØ Detector, DØ note 4079 (2003) — Cited on page 43.

[39] G. Borissov, Ordering a Chaos or Technical Details of AA Tracking, http://www-d0.fnal.gov/global_tracking/talks/20030228/ talk-adm-030228.ps (2003) - Cited on page 43.

[40] P. Hough, Machine Analysis of Bubble Chamber Pictures, Proc. Int. Conf. High Energy Accel. and Instr. (1959) — Cited on page 44.

[41] A. Khanov, HTF: histogramming method for finding tracks, DØ note 3778 (2000) — Cited on page 44

[42] A. Das et al., Fake Track Discriminant, DØ note 5948 (2009) — Cited on pages 44 and 55.

[43] M. Meijer, Using AFEII-t Time Information, DØ note 5789 (2008) — Cited on page 44.

[44] G. Gutierrez, CFT Timing Studies in Data, http://www-d0.hef .ru.nl//askArchive.php?categ=a081373\&id= a081373s1t3/transparencies (2008) - Cited on page 46.

[45] E. Varnes et al., The effect of applying a CFT timing cut at the fiber level on track reconstruction,

DØ note 5912 (2009) - Cited on page 55.

[46] A. Schwartzman and C Tully, Primary Vertex Reconstruction by Means of Adaptive Vertex Fitting,

DØ note 4918 (2005) - Cited on page 55.

[47] C. Jarvis, A study of the DØ muon detector response and an update to the loose identification and tracking efficiencies for PMCS,

DØ note 5076 (2006) — Cited on page 56.

[48] O. Brandt et al., Muon Identification Certification for the Summer 2009 Extended Dataset,

DØ note 6025 (2010) — Cited on page 56. 
[49] M. Aoki, Electron Likelihood in p20,

DØ note 5675 (2009) — Cited on page 57.

[50] EM ID group, EM ID Certification Results, http://www-d0.fnal.gov/phys_id/emid/d0_private/emid_old/ certification/main_v3_0.html (2003) - Cited on page 57.

[51] O. Atramentov et al., Electron and Photon Identification with p20 data, DØ note 5761 (2008) — Cited on page 57.

[52] U. Bassler and G. Bernardi, Towards a Coherent Treatment of Calorimetric Energies: Missing Transverse Energy, Jets, E.M. Objects and the T42 Algorithm,

DØ note 4124 (2002) — Cited on page 57.

[53] U. Baur et al., Report of the Working Group on Photon and Weak Boson Production,

FERMILAB-PUB 297 p. 115-164 (2000) — Cited on page 58.

[54] The DØ Collaboration, Determination of the absolute jet energy scale in the DØ calorimeters,

Nucl. Instr. Meth. A424 p. 352-394 (1999) - Cited on pages 59 and 60.

[55] M. Voutilainen and C. Royon, Jet $p T$ resolution using JetCorr v\%.1, DØ note 5381 (2006) - Cited on page 60.

[56] N. Makovec and J. Grivaz, Shifting, Smearing and Removing Simulated Jets,

DØ note 4914 (2005) — Cited on pages 61, 118,119 and 121.

[57] A. Schwartzman and M. Narain, Secondary Vertex Reconstruction using the Kalman Filter,

DØ note 3908 (2001) — Cited on page 62 .

[58] R. Demina, A. Khanov and F. Rizatdinova, b-tagging with Counting Signed Impact Parameter method,

DØ note 4049 (2002) — Cited on page 62 .

[59] R. Demina et al., Measurement of b-tagging efficiency and mis-tagging rates with CSIP method,

DØ note 4133 (2003) — Cited on page 62 .

[60] D. Bloch et al., Performance of the JLIP b-tagger in p14,

DØ note 4348 (2004) — Cited on page 62 . 
[61] D. Bloch and B. Clément, Update of the JLIP b-tagger Performance in p14/ pass2 with Jes 5.3,

DØ note $4824(2005)$ - Cited on page 62

[62] A. Schwartzman and M. Narain, Secondary Vertex b-tagging using the Kalman Filter Algorithm,

DØ note 3909 (2001) — Cited on page 62.

[63] T. Scanlon, A Neural Network b-tagging Tool,

DØ note 4889 (2005) - Cited on page 62.

[64] The DØ Collaboration, b-Jet identification in the DØ experiment,

Nucl. Instr. Meth. A620 p. 490-517 (2010) — Cited on pages 63 and 64

[65] B. Clément et al., SystemD, or how to get signal, backgrounds and their efficiencies with real data only,

DØ note 4159 (2003) — Cited on page 63.

[66] C. Moisan and S. de Jong, A novel method to measure $\Gamma\left(Z^{0} \rightarrow b \bar{b}\right) / \Gamma\left(Z^{0} \rightarrow\right.$ hadrons),

Conf. Proc. ICHEP-26 1 p. 670-674 (1992) - Cited on page 63.

[67] M. Anastasoaie, S. Robinson and T. Scanlon, Performance of the NN btagging Tool on p17 Data,

DØ note 5213 (2007) — Cited on pages 64, 119 and 121.

[68] T. Gadfort et al., Performance of the DØ NN b-tagging Tool on p20 Data, DØ note 5554 (2008) — Cited on page 64.

[69] The DØ $\tau$-id group, Reconstruction of $\tau$ leptons in hadronic final states at DØ Run 2,

DØ note 4210 (2003) - Cited on page 65.

[70] L. Duflot and M. Ridel, The CellNN algorithm: cell level clustering in the DØ calorimeter,

DØ note 3923 (2001) - Cited on page 65

[71] The D $\varnothing \tau$-id group, $\tau$ identification with neural networks for pass2 data, DØ note 4773 (2005) - Cited on page 66 .

[72] S. Protopopescu and P. Svoisky, $\tau$ identification with neural networks for p17 data,

DØ note 5094 (2006) — Cited on pages 67 and 83 . 
[73] F. Couderc, M. Titov and A. Patwa, Tau ID certification and systematics, http://www-d0.hef .ru.nl/fullAgenda.php?ida=a09917 (2009)

- Cited on pages 66, 117 and 121.

[74] C. Galea, S. de Jong and S. Protopopescu, Tau Energy Correction in Monte Carlo,

DØ note 5303 (2006) — Cited on page 68 .

[75] A. Duperrin, $\mathbb{E}_{\mathrm{T}}$ Significance Algorithm with p13 Run II data Application to $e+$ multijets events,

DØ note 4261 (2003) — Cited on page 68 .

[76] F. Filthaut et al., Search for SM Higgs Boson Production in the Decay $W H \rightarrow \tau \nu b \bar{b}$ Using $4.0 \mathrm{fb}^{-1}$ of Data,

DØ note 6290 (2012) — Cited on pages 71 and 109.

[77] M. Meijer et al., Search for SM Higgs Boson Production in the Decay $W H \rightarrow \tau \nu b \bar{b}$ with the DØ Detector at $s=1.96 \mathrm{TeV}$ Using $4.0 \mathrm{fb}^{-1}$ of Data, DØ note 5956 (2009) — Cited on pages 71, 109 and 132.

[78] P. Rich, Search for the Standard Model Higgs Boson in the $W H \rightarrow \tau \nu b \bar{b}$ channel with the DØ Detector,

PhD thesis University of Manchester (2009)

- Cited on pages 71, 109 and 118.

[79] J. Grivaz et al., A search for the SM Higgs boson in the $Z H \rightarrow \nu \nu b \bar{b}$ channel at $D \varnothing$,

DØ note 5568 (2008) — Cited on pages 78 and 121 .

[80] S. Calvet et al., Search for the Standard Model Higgs Boson in the ZH $\rightarrow$ $\nu \bar{\nu} b \bar{b}$ channel in $5.2 \mathrm{fb}^{-1}$ of $p \bar{p}$ collisions at $\sqrt{\mathrm{s}}=1.96 \mathrm{TeV}$,

DØ note 5857 (2010) — Cited on pages 78, 112, 117, 118 and 121.

[81] The DØ Collaboration, Search for the Standard-Model Higgs Boson in the $Z H \rightarrow \nu \nu b \bar{b}$ Channel in $8.4 \mathrm{fb}^{-1}$ of $p \bar{p}$ Collisions at $\sqrt{s}=1.96 \mathrm{TeV}$,

DØ note 6223 (2011) — Cited on page 78 .

[82] L. Wang, S. Eno and M. Sanders, Measurement of the Shape of the Boson Transverse Momentum distribution using $p \bar{p} \rightarrow Z / \gamma \rightarrow$ ee events at the Tevatron Collider,

DØ note 5187 (2007) — Cited on page 78.

[83] M. Shamim and T. Bolton, Generator Level Reweighting of $Z$ boson $p_{\mathrm{T}}$,

DØ note 5565 (2008) — Cited on pages 78 and 79. 
[84] G. Hesketh, Generator Level Reweighting of the Inclusive $W p_{\mathrm{T}}$ Distribution,

DØ note 5786 (2008) - Cited on pages 78 and 79 .

[85] G. Bernardi et al., Search for WH associated production with $5.3 \mathrm{fb}^{-1}$ of RunII data,

DØ note 5960 (2010) — Cited on page 80.

[86] The DØ Collaboration, Search for $W H$ associated production in $5.3 \mathrm{fb}^{-1}$ of $p \bar{p}$ collisions at the Fermilab Tevatron,

Phys. Rev. Lett. 698 p. 6-13 (2005) - Cited on page 80.

[87] S. Chakrabarti, P. Grannis and K. Tschann-Grimm, Search for the SM Higgs boson in the $\tau(e / \mu) \tau$ (hadrons) +2 jets final state with $4.3 \mathrm{fb}^{-1}$ of Run $2 b$ data,

DØ note 6019 (2011) — Cited on page 80.

[88] The DØ Collaboration, Search for the SM Higgs boson in the $\tau^{+} \tau^{-}+2$ jets final state,

DØ note 6171 (2011) — Cited on page 80.

[89] M. Anastasoaie, S. Robinson and T. Scanlon, nn_cert: An all in One Package for Certifying b-tagging Tools at DØ,

DØ note 5377 (2007) — Cited on page 94.

[90] K. Peters, P. Rich and S. Söldner-Rembold, Search for SM Higgs Boson production in the Decay $W H \rightarrow \tau_{h} \nu_{\tau} b \bar{b}$ with the DØ Detector at $\sqrt{s}=1.96$ $\mathrm{TeV}$,

DØ note 5669 (2008) — Cited on pages 94 and 131.

[91] B. Roe et al., Boosted Decision Trees as an Alternative to Artificial Neural Networks for Particle Identification,

Nucl. Instr. Meth. A543 p. 577-584 (2005) - Cited on page 110

[92] Y. Freund and R. Schapire, A Short Introduction to Boosting,

J. Jap. Soc. Art. Intel. 209-217 p. 771-780 (1996) — Cited on page 112.

[93] Tevatron New Phenomena \& Higgs Working Group, Combined CDF and DØ Upper Limits on Standard Model Higgs Boson Production with up to $8.6 \mathrm{fb}^{-1}$ of Data,

FERMILAB-CONF 354(2011) — Cited on pages 117, 121, 134 and 137

[94] S. Frixione and B. Webber, Matching NLO QCD computations and parton shower simulations,

JHEP 06 p. 029 (2002) — Cited on pages 117 and 121. 
[95] N. Kidonakis, Single top quark production at the Fermilab Tevatron: Threshold resummation and finite-order soft gluon corrections,

Phys. Rev. D74 p. 114012 (2006) — Cited on pages 117 and 121.

[96] S. Moch and P. Uwer, Heavy Quark pair production at two loops in QCD,

Nucl. Phys. Proc. Suppl. 183 p. 75-80 (2008)

- Cited on pages 117 and 121.

[97] G. Davies et al., General Higgs Meeting News July $9^{\text {th }}$ 2009,

http://www-d0.hef.kun.nl/fullAgenda.php?ida=a091096 (2009)

- Cited on pages 117 and 121.

[98] S. Muanza, A compilation of MCFM v5.1 Cross Sections,

DØ note 5300 (2006) - Cited on pages 118 and 121

[99] T. Andeen et al., The DØ Experiment's Integrated Luminosity for Tevatron Run IIa,

DØ note 5398 (2007) — Cited on pages 118 and 121

[100] D. Stump et al., Inclusive Jet Production, Parton Distributions, and the Search for New Physics,

JHEP 10 p. 046 (2003) — Cited on pages 118 and 121

[101] W. Fisher, Alpgen Systematics V2.0,

www-clued0.fnal.gov/ wfisher/d0_private/AlpDocs/alpgenSysts.h

- Cited on pages 119 and 121.

[102] W. Fisher, J. Haley and D. Price, Studies of Alpgen parameters, corrections and associated uncertainties,

DØ note 5966 (2009) — Cited on pages 119 and 121

[103] A. Read, Presentation of search results: the CLs technique,

J. Phys. G28 p. 2693 (2002) - Cited on page 121.

[104] W. Fisher, Collie: A Confidence Level Limit Evaluator,

DØ note 5595 (2008) - Cited on page 121.

[105] T. Junk, Confidence level computation for combining searches with small statistics,

Nucl. Instr. Meth. A434 p. 435-443 (1999) - Cited on page 124

[106] The DØ Collaboration, Search for the Standard Model Higgs Boson in Tau Final States,

Phys. Rev. Lett. 102 p. 251801 (2009) — Cited on page 131. 
[107] The DØ Collaboration, Combined Upper Limits on Standard Model Higgs Boson Production from the DØ Experiment in up to $8.6 \mathrm{fb}^{-1}$ of Data,

DØ note 6229-CONF (2011) — Cited on pages 133 and 134.

[108] The CDF Collaboration, Combined Upper Limit on Standard Model Higgs Boson Production in up to $5.9 \mathrm{fb}^{-1}$ of Data,

CDF note 10223 (2010) — Cited on pages 136 and 138.

[109] The ATLAS and CMS Collaborations, Combined Standard Model Higgs boson searches with up to $2.3 \mathrm{fb}^{-1}$ of pp collision data at $\sqrt{s}=7$ TeV at the $L H C$,

ATLAS-CONF 2011-157, CMS PS HIG 11-023 (2011)

- Cited on pages 136 and 139

[110] S. Martin, A Supersymmetry Primer,

[hep-ph/9709356v6] (1997) — Cited on page 139. 


\section{Summary}

Since its creation in the 1970s, the Standard Model (SM) has proven to be a very successful theory. It states that there is a set of fermionic fields, with specific charges for the fundamental forces: the electromagnetic, the weak and the strong interaction. The quantum excitations of these fields are seen as the matter particles. The Standard Model also postulates that nature has three fundamental symmetries, $U(1), S U(2)$ and $S U(3)$, corresponding to the fundamental forces. The symmetries give rise to gauge fields, of which the quantum excitations are called gauge bosons; photons transmit the electromagnetic interaction, $W$ and $Z$ bosons the weak interaction, and the strong interaction is carried by gluons.

If a symmetry is perfect, the corresponding gauge bosons are massless. Photons and gluons have zero mass, so the electromagnetic and strong force are caused by an exact symmetry. The $W$ and $Z$ bosons do carry mass. Therefore, the weak symmetry is somehow broken.

Explicitly breaking a symmetry would lead to a non-predictive theory. Since this is undesirable, a way to break the symmetry spontaneously was developed: the Higgs mechanism. It postulates an extra bosonic field with a fully symmetric potential but with multiple equivalent minima of this potential. The vacuum must be a single one of these lowest-energy states, so the realization of the vacuum becomes asymmetric, while the theory itself retains its symmetry.

Besides giving mass to the gauge bosons of the weak interaction, introducing the Higgs field can also lead to a mass split between fermions with different weak charges. Another consequence of an additional field is that this field can have quantum excitations, so other particles are automatically introduced. All these particles can be gauged away, except for one: the Higgs boson $H$. The Higgs field has many specific properties in order to give the particles their observed masses. This translates into specific properties of the Higgs boson: it couples to other particles proportional to their masses.

With all the other particles and their properties know, the Higgs boson's only unknown property is its own mass. Previous experiments at the Large Electron Positron collider have shown the Higgs mass $m_{H}$ is greater than $114.4 \mathrm{GeV} / \mathrm{c}^{2}$. 
Indirect measurements indicate that it is lower than roughly $200 \mathrm{GeV} / \mathrm{c}^{2}$.

To search for the Higgs boson, an environment with enough energy to create it is needed. Particle colliders are built to create such circumstances. The Tevatron in Chicago, USA, was the collider that provided the collisions used for this thesis. The Tevatron collides protons and antiprotons, which are composite particles made of (anti)quarks and gluons.

The $\mathrm{D} \varnothing$ detector is built around one of the Tevatron's collision points. Its goal is to measure the types of particles produced in the collisions, their energy and their direction. Several objects can be reconstructed, such as tracks (charged particle trajectories in the detector), jets (sprays of particles produced by hadronized quarks or gluons) and missing transverse energy (an energy imbalance in the transverse plane, which signifies the presence of neutrinos in the event).

There are several processes in which a SM Higgs boson can be made, and it can decay into different particle pairs. The detector signatures are specific for the final state. The search for the Higgs boson is therefore split into different channels. This thesis describes a search for the channel where a $W$ was produced alongside a SM Higgs boson and the $W$ decays subsequently into a neutrino and a hadronically decaying tau, while the Higgs boson decays into a $b$-quark anti-b-quark pair. In short, the channel is called $W H \rightarrow \tau \nu b \bar{b}$.

In order to search for this process, we select collisions that have a high probability of containing a neutrino in the final state. Although the neutrinos escape detection, they can be identified because they create apparent missing transverse energy. Furthermore, we require a tau in the event, of which the hadronic decays correspond to different detector signatures; tau type 1 corresponds mainly to $\tau^{ \pm} \rightarrow \pi^{ \pm} \nu$ and has clustered energy in the hadronic, but not in the electromagnetic calorimeter; tau type 2 has clustered energy also in the electromagnetic calorimeter and mostly consists of $\tau^{ \pm} \rightarrow \pi^{ \pm} \pi^{0} \nu$. Both events with type 1 and with type 2 tau-like objects are selected. The event should also have two or three jets, which are candidates for $b$-quarks. We remove events where an electron or muon is present, thereby rejecting events that are selected for other search channels.

Both background and signal are modeled using Monte-Carlo simulations. These include a description of the detector and its readout to get the most realistic modeling. The Monte-Carlo generated events are corrected for known inadequacies, such as the amount of heavy quarks ( $c$ or $b$ ) produced and detector inefficiencies.

Background due to instrumental misidentification is very hard to model using simulations, however. Therefore we use recorded data. A sideband region is formed by taking events with fake taus: objects that somewhat resemble a tau, but are not real taus. Events from this sideband region are used to model the 
instrumental, or multijet, background in the region where events with real taus are expected.

Some variables play an important role in determining whether the object is tau-like. In the sideband region (with fake taus) their distribution is different than in the signal region (with mostly real taus). The multijet model is corrected for these systematic effects with the use of an independent control sample, which has mostly fake taus, but ones that resemble more the tau-like objects in the signal region.

With the modeling fixed, we place further selection cuts. Events with a low significance of having missing transverse energy are removed. Event are also rejected if the missing transverse energy from calorimetry is inconsistent with the particle tracks. We conclude that the recorded events are reasonably well modeled after these further selection cuts.

Because the $W H \rightarrow \tau \nu b \bar{b}$ channel has two $b$-jets in the final state, we proceed with $b$-tagging to determine the $b$-ness of the jets. This is done with neural networks, where the fact is used that particles containing a $b$-quark decay after traveling a few millimeters.

For the $W H \rightarrow \tau \nu b \bar{b}$ analysis, we use either loose or tight cuts on the $b$-ness of the jets. For one sample, we select events that have two jets passing the loose criterion. Another sample contains events with one jet passing the tight criterion and the other jet explicitly failing the loose one. This configuration ensures events cannot be selected for both samples, so statistical independence is assured.

Both $b$-tagged samples are split into samples with a type 1 or a type 2 tau and with either two or three jets in the event. This results in a total of eight independent samples.

In order to best exploit the available information, Boosted Decision Trees are used for the final characterization of the event. During training on a subset of the Monte-Carlo events, they identify how signal-like or background-like regions in the multidimensional input-variable space are. Data events as well as MonteCarlo events which are not used for training are then ranked for the amount they are signal-like, creating an output distribution. A Boosted Decision Tree is trained for each sample and for each hypothesized Higgs mass from 100 to $150 \mathrm{GeV} / \mathrm{c}^{2}$ with $5 \mathrm{GeV} / \mathrm{c}^{2}$ intervals.

Pseudo-experiments form Boosted Decision Tree output distributions for the no-Higgs boson and the SM-Higgs boson hypotheses. Such an experiment takes the signal and background models and fluctuates these following Poisson distributions for the bin-by-bin statistical uncertainties and following Gaussians for the systematical uncertainties. For both hypotheses, the systematic uncertainties are fitted to the data of the pseudo-experiment by maximizing the likelihood over all samples simultaneously. The log of the ratio of the likelihoods (LLR) then indicates how much the pseudo-experiment resembled coming from the sig- 
nal+background hypothesis or the background-only hypothesis. In this way, the pseudo-experiments are used to create LLR distributions for both hypotheses. For the observed data the LLR is also determined. This procedure is repeated for every mass point.

From the LLR-distributions, the Confidence Level (CL) can be obtained; it is the probability to measure a higher LLR than the actually measured LLR. The $\mathrm{CL}_{\mathrm{S}}$ is subsequently defined as the ratio of the CL for the signal+background hypothesis over the CL of the background-only hypothesis. This means that if the observed LLR is also very incompatible with the no-Higgs scenario, it is harder to conclude that there is no Higgs boson.

Because we do not observe a significant excess of events that resemble a Higgs signal over the expected background, limits are set on the cross section of the channel. This can be done by inflating the signal cross section until it can be excluded with $95 \% \mathrm{CL}_{\mathrm{S}}$. We do this for the observed data, but also for the median of the no-Higgs pseudo-experiment distribution. The latter provides us with an 'expected limit', which signifies the sensitivity of the measurement, averaged over expected fluctuations.

For the $W H \rightarrow \tau \nu b \bar{b}$ analysis, we get an expected limit of 21.7 and an observed limit of 11.7 times the SM cross section for the Higgs mass of $115 \mathrm{GeV} / \mathrm{c}^{2}$. For Higgs masses 100 and $125 \mathrm{GeV} / \mathrm{c}^{2}$, the observed (expected) limits are 10.9 (16.1) and 14.7 (25.6) times the standard model cross section, respectively.

The DØ experiment combines several Higgs-boson channels with the condition that they are independent measurements. A sensitive channel in this combination is $Z H \rightarrow \nu \nu b \bar{b}$, which has missing transverse energy and $b$-jets as its detector signature. Because the $W H \rightarrow \tau \nu b \bar{b}$ channel also has these signatures, it is hard to remove events that are selected for $Z H \rightarrow \nu \nu b \bar{b}$ and thereby ensure independence. A rough estimate indicates $23 \pm 2 \%$ of $W H \rightarrow \tau \nu b \bar{b}$ events are also selected for $Z H \rightarrow \nu \nu b \bar{b}$.

The D $\varnothing$ combination of the summer of 2011, shows an observed (expected) limit of 1.83 (1.90) times the SM cross section for $m_{H}=115 \mathrm{GeV} / \mathrm{c}^{2}$. For Higgs masses between 161 and $170 \mathrm{GeV} / \mathrm{c}^{2}$, the observed limit is lower than the SM cross section. In this mass range the Higgs boson decays almost exclusively into $W$-pairs, a search channel that the $\mathrm{D} \varnothing$ detector is particularly sensitive to. This means DØ excludes a SM Higgs boson within that mass range, at $95 \%$ confidence level.

The D $\varnothing$ channels can also be combined with those of the other experiment at the Tevatron: CDF. This Tevatron combination has an observed (expected) limit at $m_{H}=115 \mathrm{GeV} / \mathrm{c}^{2}$ of 1.22 (1.17) times the SM cross section. The excluded mass range at $95 \% \mathrm{CL}$ then extends from 156 to $177 \mathrm{GeV} / \mathrm{c}^{2}$.

The Large Hadron Collider at CERN, Geneva, has started to contribute significantly. Its two main experiments, ATLAS and CMS, excluded the mass region 
141 to $476 \mathrm{GeV} / \mathrm{c}^{2}$ in the summer of 2011 . Only a small mass region is left compatible with both the direct and indirect measurements. This exclusion was measured with up to $2.3 \mathrm{fb}^{-1}$ of data per experiment analyzed. The full 2011 dataset is $5.2 \mathrm{fb}^{-1}$ per experiment and about $15 \mathrm{fb}^{-1}$ per experiment is expected at the end of 2012. This makes for good prospects that the SM Higgs boson will soon either be excluded or that evidence for it will be found.

In any case - whether the Higgs boson is found or not — we live in interesting times... 


\section{Populair-Wetenschappelijke Samenvatting}

Dit proefschrift beschrijft de zoektocht naar het befaamde Higgs-deeltje: het missende stukje in de puzzel van de kleinste deeltjes die we nu kennen.

Eén van de vakgebieden van de natuurkunde is de beschrijving van de allerkleinste bouwstenen van de natuur. Dit vakgebied heet de elementaire-deeltjesfysica of, omdat de meeste deeltjes zich vooral manifesteren in een omgeving met veel energie, hoge-energiefysica.

Sinds de jaren '70 van de vorige eeuw, hebben we een theorie die de onderlinge interacties van de elementaire deeltjes erg goed beschrijft: het Standaardmodel. Volgens deze theorie bestaan er velden die overal aanwezig zijn, net zoals lucht dat voor ons lijkt. De velden hebben op ieder punt in de ruimte een andere waarde, maar omdat de punten met elkaar in verbinding staan kunnen er golven ontstaan. In de luchtanalogie heeft iedere plek een luchtdruk, maar verschillen zorgen voor geluidsgolven. De velden van het Standaardmodel zijn daarnaast gekwantiseerd, wat betekent dat de trillingen een minimale grootte hebben. Zo'n kleinste - en dus ondeelbare - golf, zien we als een elementair deeltje.

Het Standaardmodel zegt ook dat de natuur drie symmetrieën heeft, die met de fundamentele krachten overeenkomen: de zwakke interactie, verantwoordelijk voor radioactief verval; de sterke interactie, die de kerndeeltjes binnen een atoom bij elkaar houdt, en de wat bekendere elektromagnetische kracht. Deze symmetrieën zelf zorgen ook voor velden, zogenaamde ijkvelden. De ijkvelden kunnen natuurlijk ook golven en de bijbehorende deeltjes zijn de krachtdeeltjes: lichtdeeltjes (fotonen) voor de elektromagnetische kracht, gluonen voor de sterke en $W$ - en $Z$-deeltjes voor de zwakke interactie. De symmetrieën van de sterke en elektromagnetische interactie blijken volledig te kloppen, wat ervoor zorgt dat de fotonen en gluonen massaloos zijn. Aangezien de $W$ - en $Z$-deeltjes massa hebben, moet de symmetrie van de zwakke interactie wel op een of andere manier gebroken zijn. Dit komt erop neer dat er voor de zwakke symmetrie in de praktijk toch een 'voorkeursrichting' bestaat. 
Het Higgs-mechanisme is het populairste model hoe de zwakke symmetrie een voorkeursrichting krijgt. Het stelt dat er een extra veld bestaat, het Higgsveld, met een potentiaal in de vorm van een sombrero; deze is symmetrisch rond het midden, maar het laagste punt zit in de rand (zie de figuur hiernaast). Het vacuüm is de toestand van laagste energie, dus in vacuüm gaat het veld ergens op de laagste rand zitten. Welk laagste punt er ook gekozen wordt, het ligt buiten het symmetrische centrum, dus er ontstaat een voorkeursrichting.

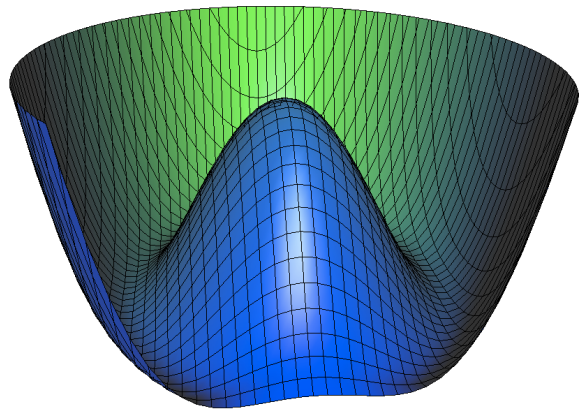

De Higgs-potentiaal. Het vacuüm kiest de laagste plek in de potentiaal, in dit geval is dat ergens in de rand.

Dat het Higgs-veld in vacuüm niet in het midden van de potentiaal zit, zorgt dat het veld een waarde ongelijk aan nul heeft. Een bijwerking is dat deeltjes zich trager door het vacuüm bewegen als ze interactie met het ontstane Higgsveld hebben. Dit zien we alsof de deeltjes massa hebben; het elektron heeft een kleine koppeling met het Higgs-veld, waardoor het makkelijk in beweging te zetten is, en we zeggen dat het elektron een kleine massa heeft. Andere deeltjes, zoals het top-quark, hebben een grote koppeling met het Higgs-veld en zijn dus zwaar. Weer andere, zoals fotonen, koppelen niet met het Higgs-veld, dus deze zijn massaloos. Door het meten van de massa weten we dus precies hoe sterk deeltjes koppelen aan het Higgs-veld.

Als het Higgs-veld bestaat, kan het zelf natuurlijk ook trillen; we noemen de bijbehorende golven Higgs-deeltjes. Omdat de koppelingen tussen het Higgs-veld en de andere deeltjes erg goed zijn bepaald, zijn die van het Higgs-deeltje met andere deeltjes dat ook. Het enige wat nog onbekend is, is de koppeling van het Higgs-deeltje met zijn eigen veld, oftewel de massa van het Higgs-deeltje zelf. Wel weten we door indirecte effecten en door er direct naar te zoeken, dat de massa hoger is dan $114,4 \mathrm{GeV} / \mathrm{c}^{2}$ en lager dan ongeveer $200 \mathrm{GeV} / \mathrm{c}^{2}{ }^{1}$

Om het Higgs-deeltje te vinden, moet een omgeving gecreëerd worden waarin genoeg energie is om het deeltje te maken, dus 115 tot $200 \mathrm{GeV}$. Daarom worden deeltjesversnellers gebruikt, zoals de Tevatron in de buurt van Chicago. De Tevatron versnelt protonen en antiprotonen tot een energie van $980 \mathrm{GeV}$ per deeltje en laat ze tegen elkaar botsen. Omdat (anti)protonen gemaakt zijn van partonen, (anti)quarks en gluonen, zijn het eigenlijk deze deeltjes die met elkaar botsen. De partonen hebben ieder maar een deel van wat de (anti)protonen aan energie in huis hebben. Vandaar dat de (anti)protonen een veel hogere energie moeten

\footnotetext{
${ }^{1} \mathrm{GeV} / \mathrm{c}^{2}$ is een maat voor de massa. Ter vergelijking: het proton, de kern van een waterstofatoom, is ongeveer $0,94 \mathrm{GeV} / \mathrm{c}^{2}$.
} 
hebben dan er nodig is om een Higgs-deeltje te maken.

Rond één van de plekken waar de protonen en antiprotonen op elkaar komen, is de $\mathrm{D} \varnothing$-detector gebouwd. Dit $20 \mathrm{~m} \times 12 \mathrm{~m} \times 12 \mathrm{~m}$ grote apparaat bestaat uit drie subsystemen die elk kijken naar een bepaald aspect van de deeltjes die tijdens de botsing geproduceerd worden. Het binnenste van de detector bestaat uit de 'tracker', die zo precies mogelijk de snelheid en richting van de ontstane geladen deeltjes meet. Direct daarbuiten zit de 'calorimeter', die geladen én neutrale deeltjes stopt en hun energie meet. Muonen - zwaardere broertjes van de elektronen - worden nauwelijks gestopt, dus als er in de 'muonkamers' rondom de calorimeter sporen van deeltjes worden gemeten, dan weten we vrij zeker dat het muonen waren. Met de drie subsystemen worden alle ontstane deeltjes gemeten, behalve neutrino's; zij hebben zo weinig kans überhaupt iets te raken, dat ze de detector volledig ongezien ontglippen. Wel kunnen we uit de energie-onbalans opmaken dat er één of meerdere neutrino's een bepaalde kant op zijn gegaan.

In een proton-antiprotonbotsing zijn er verschillende manieren om het Higgsdeeltje te maken. Na zijn ontstaan, vervalt het weer razendsnel in lichtere deeltjes (die eventueel weer vervallen) totdat er relatief stabiele en dus langlevende deeltjes overblijven. De verschillende creatie- en vervalmanieren zijn te onderscheiden in de kenmerken die ze in de detector achterlaten. Dit proefschrift richt zich op een Higgs-deeltje $(H)$ dat in combinatie met een $W$-deeltje wordt gemaakt. Het Higgs-deeltje vervalt vervolgens in een $b$-quark en -antiquark. De $W$ vervalt ondertussen in een neutrino $(\nu)$ en een $\tau$ - het zwaarste broertje van het elektron. We kijken daarbij alleen maar naar een $\tau$ dat zelf in (anti)quarks en een neutrino vervalt. In het kort heet dit het $W H \rightarrow \tau \nu b \bar{b}$-kanaal.

Onze zoektocht begint door het patroon van ons kanaal in de detector te zoeken: een neutrino zien we zoals gezegd door een grote energie-onbalans; de twee $b$-quarks zien we als zogenaamde 'jets' — waaiers van deeltjes die ontstaan als er quarks of gluonen wegschieten - en daar willen we er dus tenminste twee van hebben. Omdat de $\tau$ lastig te herkennen is, gebruiken we een combinatie van verschillende variabelen waarmee bepaald kan worden hoe $\tau$-achtig het object was. Verder letten we erop dat er geen muonen of elektronen zijn ontstaan. We willen namelijk onafhankelijk blijven van de elektron- en muonkanalen.

We willen de eigenschappen van de gemeten botsingen met die van de voorspelde achtergrond en een eventueel Higgs-signaal vergelijken. Daarvoor gebruiken we honderden miljoenen gesimuleerde botsingen, om zo de verdeling van de verschillende soorten achtergronden en het verwachte signaal te bepalen. De simulaties worden gedaan met behulp van de uitgebreide theoretische kennis van de bekende achtergrondprocessen en de koppelingen van een mogelijk Higgs-deeltje. Van de ontstane deeltjes uit de gesimuleerde botsingen wordt ook bekeken hoe een digitaal model van de detector inclusief elektronica erop reageert. Hierdoor worden de gesimuleerde achtergronden zo realistisch mogelijk. 
Verreweg de meeste botsingen behelzen alleen de sterke interactie. Normaliter komen die niet door de genoemde selectieprocedure, vooral omdat ze geen neutrino's produceren. Door meetfouten in de detector wordt zo'n botsing soms wel degelijk als interessant aangemerkt. De achtergrond die hierdoor ontstaat is erg lastig te simuleren, maar hij is wel groot genoeg om van belang te zijn. We noemen deze achtergrond 'multi-jet', omdat hij in werkelijkheid alleen uit een aantal jets bestaat. De multi-jet-achtergrond modelleren we door gemeten data. Hiervoor nemen we een monster van botsingen waarvan er maar weinig een $\tau$ bevatten, omdat dit monster voor de overgrote meerderheid uit multi-jetachtergrond bestaat. Vervolgens wordt dit monster vertaald naar het signaalmonster, wat bestaat uit botsingen die waarschijnlijk een $\tau$ produceerden. Bij dit vertalen wordt het verschil van de monsters in de totale hoeveelheid multijet-achtergrond meegenomen, maar ook het verschil in verdelingen van de twee belangrijkste $\tau$-eigenschappen: zijn snelheid en richting.

We hebben met verschillende controlemonsters bekeken of de gesimuleerde en multi-jet-achtergronden overeenkwamen met de gemeten data. Hierna hebben we de selectie aangescherpt door van de botsingen in het signaalmonster te eisen dat de waarneming van een grote energie-onbalans relatief zeker is. Verder moet de openingshoek tussen de energie-onbalans in de calorimeter en die in de tracker niet te groot zijn, omdat ook dat zou kunnen duiden op een instrumentele fout. Tenslotte nemen we alleen botsingen mee waarvan op zijn minst één van de jets afkomstig lijkt te zijn van een $b$-quark. Net als bij de $\tau$-herkenning wordt hierbij gebruik gemaakt van verschillende variabelen. Een voorbeeld daarvan is of een aantal deeltjes in de jet buiten de botsing ontstaan is. Een $b$-quark kan zich namelijk eerst een stukje verplaatsen voor het vervalt, waardoor de uiteindelijke deeltjes niet direct van de botsing zelf afkomen.

$\mathrm{Na}$ de volledige selectieprocedure houden we een monster met 570 gemeten botsingen over, terwijl we er 578 aan achtergrond en eventueel 2 aan signaal verwachten. ${ }^{2}$ Om beter te bepalen of er af en toe Higgs-deeltjes in die botsingen zaten, gebruiken we 15 verschillende eigenschappen. De belangrijkste variabele is de gezamenlijke massa van de twee jets; de jets zouden namelijk afkomstig kunnen zijn van het Higgs-deeltje, dus hun gezamenlijke massa is de mogelijke Higgs-massa. Ook bijvoorbeeld de hoek tussen de $\tau$ en het neutrino zeggen iets over de waarschijnlijkheid dat er een Higgs-deeltje bij de botsing gemaakt was. Deze variabelen worden door middel van een algoritme gecombineerd tot één variabele; zeg maar de 'Higgs-achtigheid'. We kunnen dit doen door naar de eigenschappen van de gesimuleerde botsingen te kijken en te vergelijken waar er verschillen zitten tussen de signaal- en de achtergrondbotsingen. Dit is voor iedere mogelijke Higgs-massa anders, omdat het signaal er iets anders uitziet.

\footnotetext{
${ }^{2}$ Door statistische fluctuaties zijn bij een voorspelling van 578 alle waarden tussen 554 en 602 erg redelijke uitkomsten.
} 
Daarom is de Higgs-achtigheid voor iedere massa een andere combinatie van de 15 variabelen.

Met de verdelingen van de gemeten en gesimuleerde Higgs-achtigheid in de hand, kunnen we zeggen met welke mate van betrouwbaarheid we het bestaan van een Higgs-deeltje kunnen uitsluiten of bevestigen. Statistische onzekerheden worden hierin meegenomen, maar ook zogenaamde systematische onzekerheden, onzekerheden die het gevolg zijn van de manier van meten, onbekende detectorimperfecties of theoretische onzekerheden.

Helaas hebben we geen duidelijk genoeg signaal kunnen onderscheiden. Daarom worden verschillende onafhankelijke productie- en vervalskanalen binnen het DØ-experiment gecombineerd. Dit verhoogt de gevoeligheid voor een Higgsdeeltje sterk. Een van de belangrijkste kanalen daarin is $Z H \rightarrow \nu \nu b \bar{b}$ waar het Higgs-deeltje ook vervalt in $b$-quarks, maar nu geproduceerd wordt naast een $Z$ deeltje dat vervalt in twee neutrino's. Dit kanaal heeft een energie-onbalans en twee $b$-jets als selectiecriteria. Dit eisen we ook voor ons $W H \rightarrow \tau \nu b \bar{b}$-kanaal, en het is dus erg lastig op voorhand botsingen die het andere kanaal gebruikt uit te sluiten. Hierdoor is onze meting niet meer onafhankelijk van die van het andere kanaal en kan ons kanaal dus jammer genoeg niet makkelijk gebruikt worden voor de $\mathrm{D} \varnothing$-combinatie. Om een idee te geven: ongeveer een kwart van de botsingen binnen ons kanaal blijken ook gebruikt te worden voor de $Z H \rightarrow \nu \nu b \bar{b}$-meting.

Naast de $D \emptyset$-combinatie is er ook een Tevatron-combinatie gemaakt. Hiervoor zijn de resultaten van $\mathrm{D} \varnothing$ gecombineerd met die van CDF, het andere experiment dat gebruik maakt van de Tevatron-versneller. Door deze samenvoeging is de gevoeligheid voor een Higgs-signaal veel groter. In totaal kunnen we daardoor tussen de massa's 156 tot $177 \mathrm{GeV} / \mathrm{c}^{2}$ het verwachte signaal uitsluiten met een $95 \%$ betrouwbaarheid. Aan de andere kant zien we tussen 125 en $130 \mathrm{GeV} / \mathrm{c}^{2}$ iets wat met $92 \%$ betrouwbaarheid een signaal zou kunnen zijn. Voor ons vakgebied is dat niet voldoende; voor een zogenaamd 'bewijs' is $99,7 \%$ nodig en voordat iets een 'ontdekking' genoemd mag worden moet er 99,99994\% betrouwbaarheid zijn.

In Europa zijn de ontwikkelingen ondertussen doorgegaan; sinds het begin van 2011 produceert de LHC — de $27 \mathrm{~km}$ lange versneller in de buurt van Genève - erg veel botsingen met per stuk een hogere energie dan die van de Tevatron. Hierdoor wordt verwacht dat de experimenten van de LHC binnen een jaar (bewijs voor) het Higgs-deeltje zullen vinden, of het bestaan ervan zullen uitsluiten. Vooral dat laatste zou een enorme paradigmaverandering in de natuurkunde van de allerkleinste deeltjes betekenen.

Hoe dan ook — of het Higgs-deeltje gevonden gaat worden of juist niet — we leven in spannende tijden... 


\section{Acknowledgements}

In this section I would like to thank the many people that helped me to write this thesis and with the work prior to it.

First of all I want to thank my supervisors Sijbrand and Frank for their continued support. I found myself very inspired after many a visit with Sijbrand, and Frank motivated a lot of the perspiration. Together they were more than crucial for the completion of the analysis and the resulting thesis.

The previous version of my analysis was developed by Philip Rich, to whom I owe many thanks for explaining me all the computational steps of the analysis both online and during my visits at The University of Manchester.

A very important part of work is the people one works with. Thanks to Harm and Stefan Grebe for sparring with me on all sorts of physics and other kind of problems, even if it kept us from the work we were supposed to be doing. Also many thanks to Geert-Jan and Marcel who (re)read every part of this thesis until they could not find any more of the obvious errors that I overlooked. Of course the many times we went cycling and had a beer or two with Thijs were a very welcome way to relieve ourselves of the (programming) troubles of the day too...

I really enjoyed the lunches for the discussions with and company of everybody, including Irene, José, Yiannis, Gijs, Jari, Stefan Jansen, Guus; my office mates Lucian, Folkert, Magda, Antonio and Eric; all the bachelor and master students and the staff at the high energy physics departments. I think it is a rare thing to have such a very good and proactive atmosphere at work. Everyone in the departments was responsible for this and deserves credit, but I would especially like to thank Annelies, Gemma and Marjo for making this possible and not getting annoyed when there were noisy conversations in their secretariat.

Of course I found support with many of my friends outside of work, Floris and Lucy, Crispijn and Michelle, Lisa, Joke en Rik, Jorrit, Lyda, Jan, Sytske and Sandor, Dorine and Bert, Annemieke and Mattias. With many of you we go back so long I find it hard to imagine how I could not have known you. Thank you also for your patience when I rambled on about physics for a bit too long. 
I also owe my family a lot of gratitude. My father for teaching me to keep questioning, my mother for teaching me to think of others, Bert for showing there is a different way of thinking, Daan for the growing respect we have for each other, Joline for being such a calm and very kind addition to the family. I would also like to thank my family-in-law for opening your hearts to me, at least that is how I felt it. But to all of you I would like to say it is a miracle that you allowed me to endlessly go on about tiny particles and that you never gave up hope that I would turn out ok.

But most thanks go out to my lovely girlfriend and future wife, Linda. I truly would not know what to do without you! And you always made sure I finished before a deadline, not after, as I would have done. I am not sure where we both will be in a year from now, but sticking together I am certain the future will be more than great. 


\section{Curriculum Vitae}

The author of this thesis, Melvin Michaël Meijer,

studied physics in Nijmegen. As part of his Master's degree in theoretical high energy physics, he stayed for almost a year at Stony Brook University, New York, to do research with professor Jack Smith. Under supervision of professor Ronald Kleiss, he graduated with the thesis "Helicity calculation of charmonium production matrix elements with longitudinally polarized gluons and photons".

After a short stay at CERN, Geneva, to help in the muon-identification for CMS, he started his PhD project in experimental high energy physics with professor Sijbrand de Jong and Frank Filthaut, PhD. His research on the Standard Model Higgs boson at the DØ detector at Fermilab, Chicago, led to the writing of this thesis. 